\title{
AN ECONOMIC ANALYSTS OF THE KILAUEA GEOTHERMAL DEVELOPMENT AND \\ INTER-ISLAND CABLE PROJECT
}

Northwest Economic Associates 13101 N.E. Highway 99 Suite 200 Xancouyer, Washington $98686-2786$

$$
\text { +4ty }
$$

anchent:

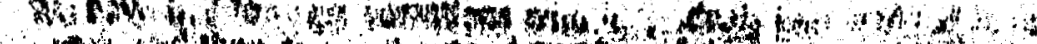

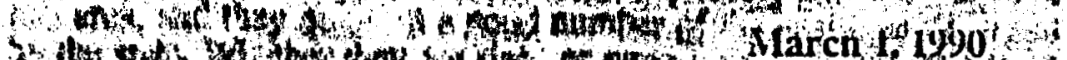

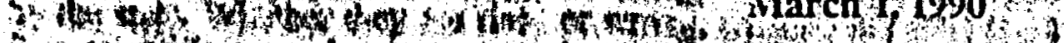

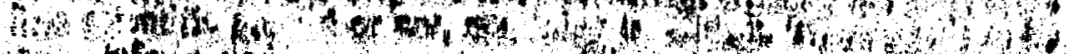

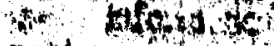

Dos

$+6+8$

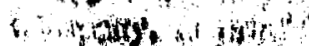

$\therefore$ istrind

Kont wate of

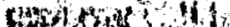

Finte

and indin

ats $\mathrm{Lat}$

rusendy in

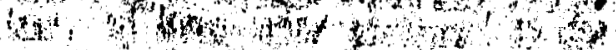

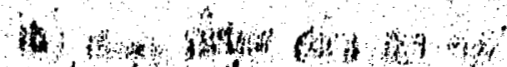

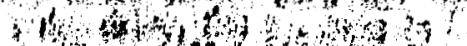

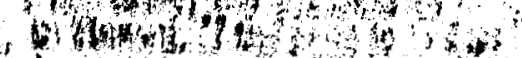
3 mo ind कy

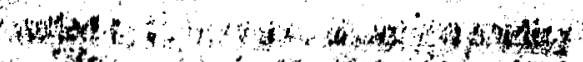

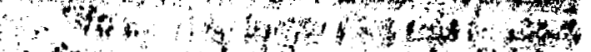

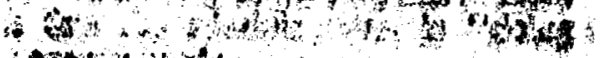
Aatentas 


\section{DISCLAIMER}

This report was prepared as an account of work sponsored by an agency of the United States Government. Neither the United States Government nor any agency Thereof, nor any of their employees, makes any warranty, express or implied, or assumes any legal liability or responsibility for the accuracy, completeness, or usefulness of any information, apparatus, product, or process disclosed, or represents that its use would not infringe privately owned rights. Reference herein to any specific commercial product, process, or service by trade name, trademark, manufacturer, or otherwise does not necessarily constitute or imply its endorsement, recommendation, or favoring by the United States Government or any agency thereof. The views and opinions of authors expressed herein do not necessarily state or reflect those of the United States Government or any agency thereof. 


\section{DISCLAIMER}

Portions of this document may be illegible in electronic image products. Images are produced from the best available original document. 


\section{TABLE OF CONTENTS}

Page

HIGHLIGHTS AND CONCLUSIONS__ i

INTRODUCTION _ ii

THE GEOTHERMAL PROJECT AS CURRENTLY ENVISIONED___ 1

THE COST OF THE GEOTHERMAL PROJECT AS

CURRENTLY ENVISIONED _ _

NEA - GEOTHERMAL PROJECT COST ESTIMATES

Geothermal Power Plants___ 9

Transmission Cable System _ 15

Submarine Transmission Cables__ $\quad 15$

Overhead Transmission Lines and Facilities_ 20

NEA - OIL AND SOLAR/OIL POWER PLANT COST ESTIMATES__ $\quad 23$

Oil Plants__ 23

Solar/Oil Plants____ 24

NEA - GEOTHERMAL PROJECT HAZARD ASSESSMENT___ $\quad 27$

NEA - PROJECT INTEREST RATES__ '

NEA - PROJECT INSURANCE RATES_

NEA - THE PRICE OF RESIDUAL FUEL OIL__ $\quad 49$

NEA - PROJECT COST COMPARISON

Geothermal Power Generation__ $\quad 59$

Residual Fuel Oil Power Generation__ $\quad 60$

Solar/Residual Fuel Oil Power Generation__ 61

Scenario 1

Scenario 2_ $\quad 69$

Scenario 3— 75

Scenario 4__ 81 


\section{TABLE OF CONTENTS}

cont'd

- Page

NEA - CONSERVATION AND SOLAR ENERGY__ 93

NEA - CONCLUSIONS_____________ 99

\section{APPENDICES}

Appendix A - Well Cost

Appendix B - Geothermal Plant Costs

Appendix C - Criticism, Review and Comments on The February, 1988 Decision Analysts

Hawaii. Inc. Report

Appendix D - Robert J. Mowris Report

Appendix E - Costs If Production Well Increases To

200\% Replacement 


\section{HIGHLIGHTS AND CONCLUSIONS}

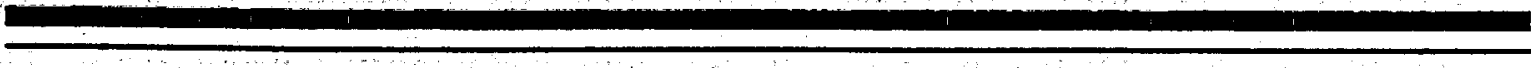

A study by NEA completed in April 1987 shows that a large scale ( $500 \mathrm{MW})$ geothermal development on the big island of Hawaii and the inter-island power transmission cable is economically infeasible. This updated report, utilizing additional information available since 1987 , reaches the same conclusion:

- The state estimate of $\$ 17$ billion for development cost of the geothermal project is low and extremely optimistic. More realistic development costs are shown to be in the range of $\$ 3.4$ to $\$ 43$ billion and could go as high as $\$ 4.6$ billion.

- Compared to alternative sources of power generation, geothermal can be 17 to 24 times as costly as oil, and 12 to 17 times as costly as a solar/oil generating system.

- Yearly operation and maintenance costs for the large scale geothermal project are estimated to be 44.7 million, $72 \%$ greater than a solar/oil generating system.

- Over a 40-year period ratepayers could pay. on average. between 13 (172\%) and 2.4 cents (33\%) per $\mathrm{kWh}$ per year more for electricity produced by geothermal than they are currently paying (even with oil prices stabilizing at $\$ 45$ per barrel in 2010).

- A comparable solar/oil thermal energy development project is technologically feasible, could be island specific, and would cost $20 \%$ to $40 \%$ less than the proposed geothermal development.

- Conservation is the cheapest alternative of all, can significantly reduce demand, and provides the greatest return to ratepayers.

There are better options than geothermal. Before the State commits the people of Hawaii to future indebtedness and unnecessary electricity rate increases, more specific study should be conducted on the economic feasibility, timing, and 
magnitude of the geothermal project. The California experience at The Geyers points up the fact that it can be a very risky and disappointing proposition. The state should demand that proponents and developers provide specific answers to geothermals troubling questions before they make an irreversible commitment to it.

The state should also more carefully assess the potential risks and hazards of volcanic disturbances, the degree of environmental damage that could occur, the future demand for electricity, and the potential of supplying electricity from alternative energy sources, conservation and small scale power units. As we stated in the April 1987 study, to move ahead with rapid large scale geothermal development on Hawaii without thoroughly studying these aspects of its development is ill-advised and economically unsound. 


\section{INTRODUCTION}

In early 1989 the Pele Defense Fund requested that Northwest Economic Associates (NEA) update their 1987 economic analysis of the proposed Hawaii Geothermal Development And Inter-island Cable Transmission System Project. This report, the result of that request, once again compares the cost of building and operating $500 \mathrm{MW}$ of geothermal power plants and a cable transmission system with the cost of building and operating $500 \mathrm{MW}$ of oil-fired power plant generating capacity. This update also compares the geothermal project with a solar/oil hybrid generating system of the same size. The impact of energy conservation is also considered in terms of its potential as an ezergy resource and in its contribution to an energy development program for the state of Hawaii.

This report develops low and high cost estimates for the project. In a project laced with as much uncertainty as this, a single cost figure is of little value. A range of values which attempts to account for some of the uncertainty seems a more logical approach to cost estimation.

Our cost estimates include costs which should have been considered in the February 1988 Decision Analysts Hawaii, Inc. report for the State, but were not. The most obvious being a standard project cost contingencv. Dther costs not considered in the State report but included in this report are:

1) additional cost of the undersea cable to allow for slope changes on the sea floor,

2) cost of constructing the cable laying vessel which does not yet exist,

3) cost of helper vessels to assist in the cable laying, and

4) adequate insurance or plant replacement costs.

A cost that neither the State report nor this report includes, but one that may be very important, is the cost of designing or protecting the plants to deal with geologic hazards. The added cost of strengthening plants, designing them for 
quick disassembly, or constructing protective barriers around them will be considerable.

This report considers project costs as objectiveiy as possible consicering the great deal of uncertainty and high level of risk involved in the project.

All costs are shown in 1990 dollars.

Appenix C contains two criticisms of the February 1988 Decision Analysts Hawai, Inc report on the proposed geothermal project. 


\section{THE GEOTHERMAL PROJECT AS CURRENTLY ENVISIONED}

The basic project scenario is that $500 \mathrm{MW}$ of geothermal power will be generated in the Puna District on the Island of Hawaii. The power will be transmitted by overhead cables across the island to its northern tip where it will enter a submarine cable for transmission across the Alenuihaha Channel to the Island of Maui. The power will then be carried by overhead transmission lines to the southwest corner of Maui where it will enter a second submarine cable and will be transmitted along the Auau Channel and across the Kaiwi Channel to the Island of Oahu for distribution to customers. Figure 1 shows the described rcute. This is the route that is used in our analysis. Distances and channel depths are shown in Table 1

The amount of geothermal power production envisioned for the project is on the order of 500 net MW (megawatts). Allowing for transmission losses (10\%) and adequate power reserve, (20\%) 600 gross $\mathrm{MW}$ must be proven to exist and developed to meet project requirements.

The 500 net MW of power would be provided by a number of geothermal power plants located in the area of the East Rift Zone of Kilauea volcano (Figure 2). The number of plants that will be required to produce the 500 net MW would either be ten $55 \mathrm{MW}$ plants, twenty $275 \mathrm{MW}$ plants, or some combination of the two proposed sizes. The distribution and location of the plants and their wellfields will be governed by the distribution and location of the resource, but since no proof has yet been established that the necessary 600 gross MW actually exists, no attempt has been made to show a likely plant distribution pattern. In conjunction with the power plants and wellfields are the transmission cables (overhead and underwater) and their associated facilities. A general list of the system components is found in Table 2. 


\section{Figure 1}

\section{PREFERRED ROUTE \\ FDWC PROGRAM}

(N)

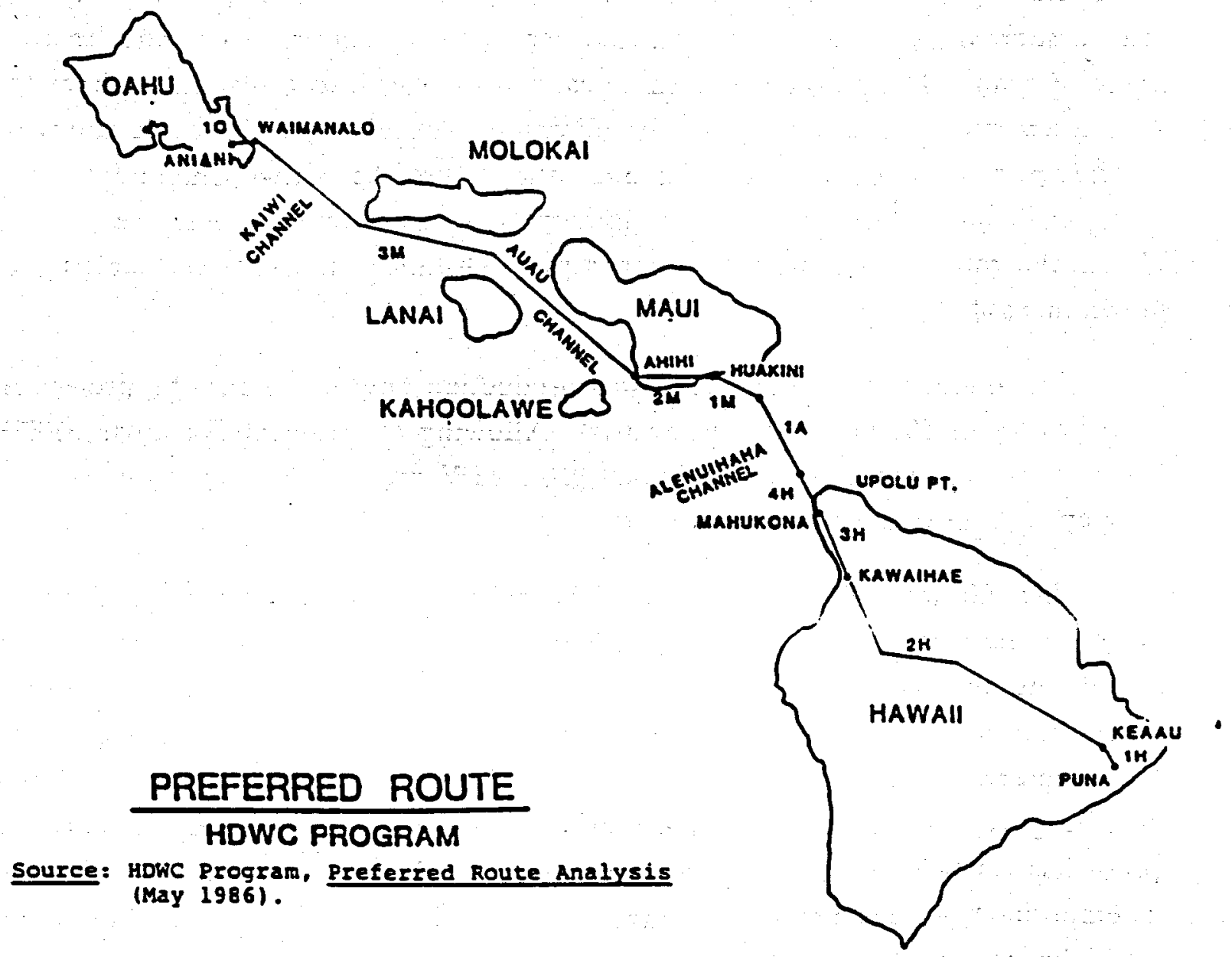

Source: $\quad$ HDWC Program, Preferred Route Analysis (May, 1986)

NEA

Source: Alternative Approaches to the Legal. Institurional. and Financial Aspects of Developing an Inser-isiand Electrical Transmission Cable System, State of Hawaii, Department of Planning and Economic Development, April, 1986. 
Table 1

DISTANCE AND DEPTH CHARACTERISTICS

OF PREFERRED ROUTE,

April, 1986

Hawaii to Maui to Oahu

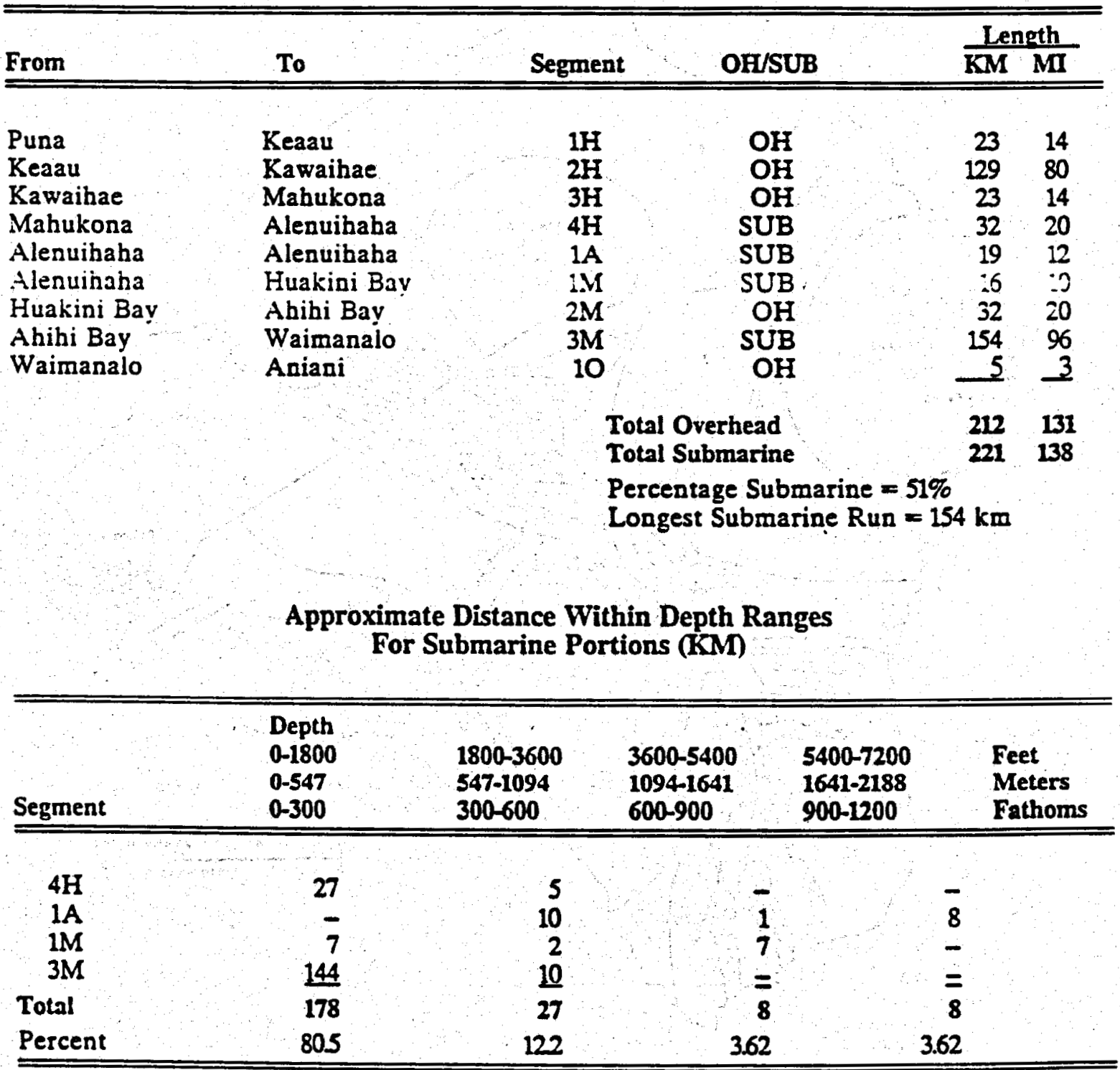

suarce: HDIVC Program. P'reterted Roure Inalusis (May, iUS6).

NEA

Source: Alternative Approaches to the Legal, Institurional, and Financial

Aspects of Developing an Inter-island Electrical Transmission Cable

System, State of Hawaii. Department of Planning and Economic

Development, April, 1986. 
Figure 2

\section{AREA OF PROPOSED GEOTHERMAL FACILITIES}

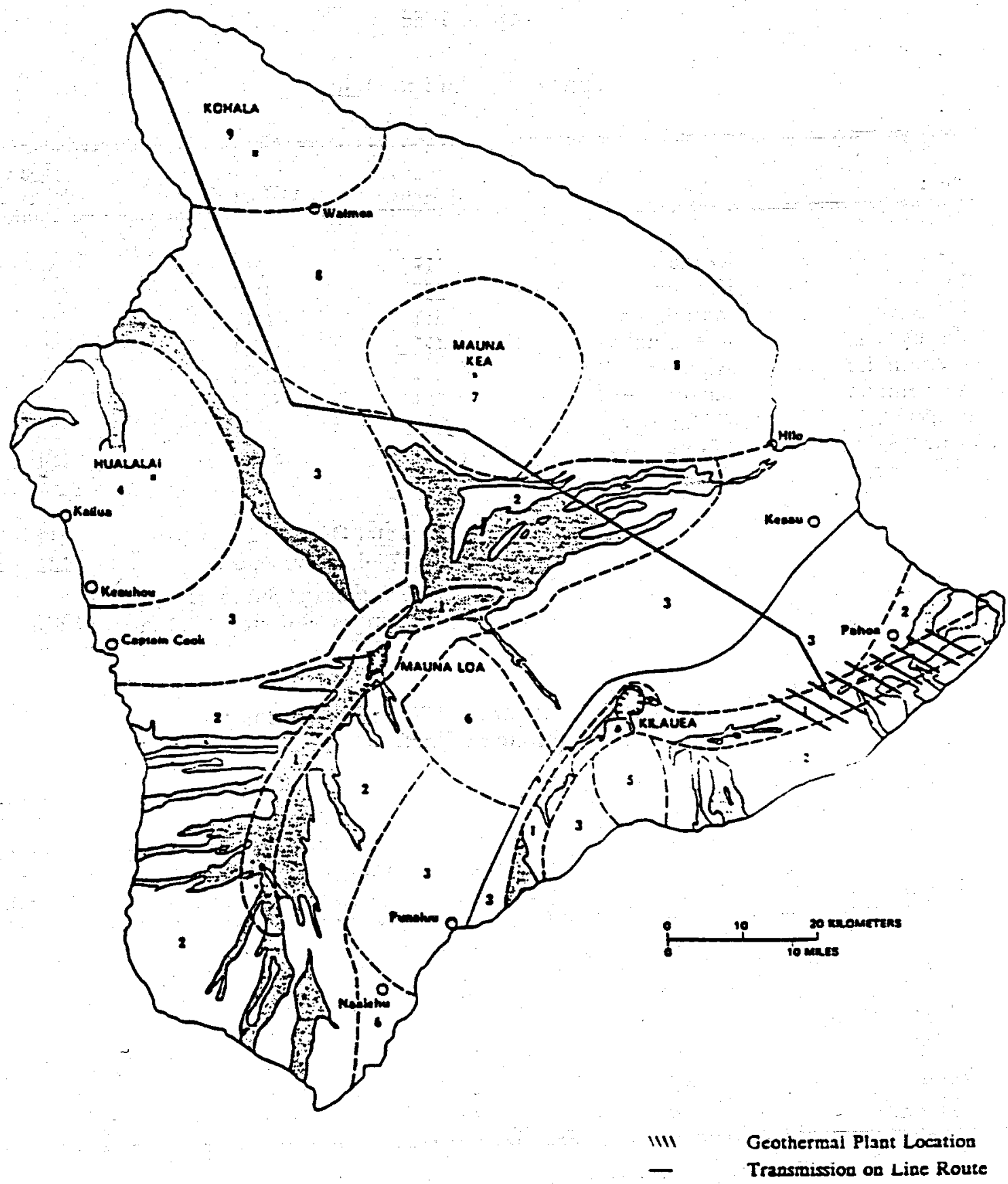

Source: Volcanism In Hawaii U.S. Geological Survey Professional Paper 1350. 
Table 2

CABLE SYSTEM FACILITIES

A cable system's facilities would consist, in the general order of sequence from the energy generation source, of the following components:

- The interconnection facilities (alternating current) to transmit the renewable alternate energy-generated electric energy from the power plants to the cable system's converter station on the Island of Hawaii;

- The land-based converter station on the Island of Hawaii to convert alternating current (ac) to direct current (dc) for cable riansmission:

- The overhead transmission line traversing the Island of Hawaii to the land-based cabie termination facility, including an oil pressurization station;

- The submarine cable system to Maui;

- The land-based oil repressurization station on Maui;

- The submarine cable system to Oahu;

- A land-based cable termination facility on Oahu;

- The overhead hvdc transmission line from the cable termination facility to the converter station on Oahu;

- The converter station to convert de to ac for interconnection to HECO's grid system; and

- The interconnection facilities to transmit electric energy from the cable to HECO's grid system.

NEA

Source: Alternative Approaches to the Legal, Institutional, and Financial Aspects of Developing an Inter-island Electrical Transmission Cable Sustem. State of Hawaii. Department of Planning and Economic Development, spril. i!\$6. 


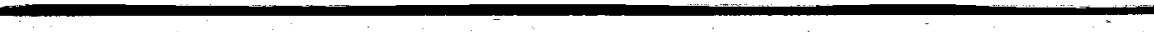

.

$\ldots \ldots$

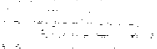

i

$\left(\begin{array}{c}2 \\ -1\end{array}\right.$ 


\section{THE COST OF THE GEOTHERMAL PROJECT AS CURRENTLY ENVISIONED}

In February, 1988, Decision Analysts Hawaii, Inc. (DAHI) submitted a report on the economic feasibility of the $500 \mathrm{MW}$ geothermal project. It estimated the development cost at 1675 billion (1986) dollars. Actual bids on the project have not been made public but speculation in the news media places them at above the $\$ 3$ billion mark. If these estimates prove accurate, they show the DAHI cost estimate to be more than a billion dollars low.

Table 3

\section{PROJECT DEVELOPMENT COST COMPARISON}

\begin{tabular}{|c|c|c|c|c|}
\hline $\begin{array}{l}25 \text { Net MW Plants } \\
\text { Capital Cost Comparison (MS) }\end{array}$ & $\begin{array}{l}\text { Plant/Wellfield } \\
\text { Surface Facilities }\end{array}$ & Wells & Cables & $\begin{array}{l}\text { Total } \\
\text { Cost }\end{array}$ \\
\hline $\begin{array}{l}\text { Without Contingency: } \\
\text { DAHI } 1986 \$ \\
\text { DAHI } 1990 \$ \text { \& } \\
\text { NEA 1990\$ (low estimate) } \\
\text { NEA } 1990 \text { \$ (high estimate) }\end{array}$ & $\begin{array}{r}6622 \\
700.9 \\
984.6 \\
1104.6\end{array}$ & $\begin{array}{l}600.0 \\
600.0 \\
675.0 \\
900.0\end{array}$ & $\begin{array}{l}413.3 \\
444.4 \\
561.4 \\
5614\end{array}$ & $\begin{array}{l}1675.5 \\
17453 \\
22210 \\
2566.0\end{array}$ \\
\hline $\begin{array}{l}\text { With } 20 \% \text { Contingency: } \\
\text { DAHI } 1990 \text { \$ } \\
\text { NEA } 1990 \$ \text { (low estimate) } \\
\text { NEA } 1990 \$ \text { (high estimate) }\end{array}$ & $\begin{array}{r}8411 \\
1345.9 \\
1507.9\end{array}$ & $\begin{array}{r}720.0 \\
810.0 \\
1080.0\end{array}$ & $\begin{array}{l}533.2 \\
-75.5 \\
073.5\end{array}$ & $\begin{array}{l}2094.3 \\
2920.4 \\
32614\end{array}$ \\
\hline $\begin{array}{l}\text { With } 20 \% \text { Contingency \& Repla } \\
\text { DAHI } 1990 \text { S } \\
\text { NEA } 1990 \text { (low estimate) } \\
\text { NEA } 1990 \text { S (high estimate) }\end{array}$ & $\begin{array}{l}\text { Wells: } \\
8411 \\
1345.9 \\
1507.9\end{array}$ & $\begin{array}{l}1440.0 \\
1620.0 \\
2160.0\end{array}$ & $\begin{array}{l}533.2 \\
673.5 \\
673.5\end{array}$ & $\begin{array}{r}2814.3 \\
3639.4 \\
43414\end{array}$ \\
\hline
\end{tabular}

11 Star-Bulletin, Special Report: Geothermal-A Heated Issue. by Susan Manuel, January 2. 1990.

$\because \quad$ The $14 \$ 6$ DAHI cost tigures are brougit up (1) iv(x) levels thing intiation rates calculated from indexes found in the Statistical Abstract of The Unised States. 109th Edition, U.S. Dept. of Commerce. The annual construction cost inflation rate used for plant/well field surface facilities is $143 \%$ based on Handy-Whitman public utility indexes for electric light and power plant construction. The annual manufacturing cost inflation rate for the cable portion of the project is $1.83 \%$ based on the producer price indexes for machinery and equipment. 



\section{NEA - GEOTHERMAL PROJECT COST ESTIMATES}

This section describes how the geothermal project cost figures were developed for use in the analysis. Costs were developed for the construction of 25 net MW and 50 net MW geothermal power plants, the manufacture and laying of submarine cable, and the construction of overhead transmission lines and associated facilities. In estimating this cost NEA used available information from various Federal, State and Private industry sources and reports, and have followed the route and system requirements found in the DAHI and previous NEA reports.

\section{GEOTHERMAL POWER PLANTS}

Costs were developed for two sizes of power plants, 25 net MW (275 gross MW) and 50 net MW ( 55 gross MW). The DAHI report uses $25 \mathrm{MW}$ plants in its analysis but indicates that current conjecture is for ten $50 \mathrm{MW}$ plants. To estimate power plant costs we used the CENTPLANT computer program. This program was created specifically for estimating geothermal development costs and is designed to estimate the capital costs of geothermal plant and wellfield surface facilities having 10 to $100 \mathrm{MW}$ capacity. The estimates are based upon the temperature of the resource, its flow rate, and the location and difficulty of developing the resource. CENTPLANT was originally developed by the Oregon Department of Energy with cost information and engineering formulas provided by Bechtel National, Inc. A copy of the program is available from the Oregon Department of Energy or the Bonneville Power Administration.

The CENTPLANT program develops costs for the following components:

Geothermal Power Plant

Turbine inlet valves and strainers

Turbine and generator

Condenser (surtace type)

Condensate pumps

Cooling towers

Circulating water pumps and piping

Main transformer

Switchyard

Process piping 
Geothermal Power Plant - cont'd

Plant electrical equipment

Instrumentation and controls

Site preparation

Turbine and control building

Balance of plant systems

Construction labor

Indirect field costs (temporary construction

facilities, miscellaneous construction services, construction equipment and supplies, field of fice, preliminary checkout and acceptance testing, and startup).

\section{Wellfield}

Production wellpad piping and equipment downstream from wellhead shutoif valves

Production wellpad instrumentation and controls

Steam or hot water transmission pipelines

Flash tanks (flashed steam plants only)

Steam release facility

Startup system for production wells (flashed steam plants only)

Reinjection pumps

Reinjection pipeline.

Reinjection piping and equipment

Reinjection instrumentation and controls

Wellfield electrical system

Wellfield distributed digital automatic control system

Construction labor

Engineering, procurement, and construction management

Indirect field costs

Production and reinjection island development

(clearing, grubbing, grading, etc.)

On-site roads

\section{Inputs Provided By Program User}

H2S Abatement

Permits and licenses

Resource assessment and exploration

Production, reinjection, and replacement wells

Owner's engineering, administrative, and general costs

Cost overrun contingency 


\section{Costs Not Included In Program}

\section{Land or land use costs}

Research and development costs

Power transmission lines beyond the AC/DC converte: station

The program generates low, mid and high estimates of costs based on builtin engineering factors provided by Bechtel National, Inc and variable input assumptions provided by the user. The low range estimate does not account for reinjection wells which will be required in the proposed $500 \mathrm{MW}$ project Therefore, only the mid and high estimates are used in this report. The mid estimate is now referred to as the low estimate using injection wells.

\section{Input Assumptions}

Low Estimate (with injection wells)

- Site preparation and construction camp is required

- 13e/kWh electricity cost at plant

- 10 miles AC transmission line

- $\$ 25$ million in remaining resource assessment work

- $\$ \mathbf{7 5 0 , 0 0 0}$ for various permits and licenses

- Wells produce at $4 \mathrm{MW}$ per well

- $2 / 1$ Production/Injection well ratio

- $3 / 1$ well drilling success ratio

- $100 \%$ production well replacement over life of the plant

High Estimate (with in jection weils)

- Site preparation and construction camp is required

- $13 \mathrm{e} / \mathrm{kWh}$ electricity cost at plant

- 10 miles AC transmission line

- $\$ 3$ million in remaining resource assessment work

- \$I million for various permits and licenses

- Wells produce at $3 \mathrm{MW}$ per well

- $2 / 1$ Production/Injection well ratio

- $3 / 1$ well drilling success ratio

- $100 \%$ production well replacement over life of the plant

Production wells are assumed to be $6,000-7,000$ feet deep and to cost $\$ 25$ million per well. Injection wells and drywells are estimated to cost $\$ 20$ million per well. The same well costs are used as those found in the DAHI report. See Appendix A for the range of well costs considered. 
This study's production to injection well ratio is 2 to 1 The DAHI report assumes a 2.67 to 1 ratio. Based on data for geothermal wells actually drilled in Japan appearing in the Geothermal Resources Council Bulletin of October 1989. a ratio of 11 to 1 was experienced. For lack of better information our ratio rests comfortably between the two.

The well drilling success ratio is 3 to 1 , while the DAHI report assumes a 4 to 1 ratio. The Japanese experience indicates a ratio of 24 to 1 The figure used in this study between the two.

The well replacement rate is $100 \%$ over 20 years, the DAHI report rate is also 20 years. Recent reports from The Geysers in California, however, indicate that steam pressure in the wellfields is falling rapidly. and that well life may te only 10 to 15 years instead of the earlier predicted 20 to 30 years. If this proves true for Hawaii, the replacement rate may be $200 \%$ on more.

Hydrogen Sulfide $\left(\mathrm{H}_{2} \mathrm{~S}\right)$ abatement assumes the use of a Stretford system. The cost is based on a similar cost from The Geysers, Unit 21 abatement system. Unit 21 is $125 \mathrm{MW}$ so the cost has been scaled down to $25 \mathrm{MW}$ and $50 \mathrm{MW}$ using the 0.6 scaling factor found in the DAHI report and brought up to 1990 dollars. According to a 1985 review of a report estimating abatement costs for Hawaii, Thermal Power Company and Bechtel Group Inc, indicated that abatement costs in Hawaii will vary widely since the resource is so variable and wiil change aver time. The estimated abatement costs reviewed by Thermal Power and Bechtel were based on a single set of assumptions and were characterized as being "at the extreme low end of published values for similar plants," such as The Geysers Unit 21.

Based on this criticism and the assumption that geothermal fluids in Hawaii are at least as toxic and corrosive as those in California, the Unit 21 abatement cost figure is used as the low abatement cost for $25 \mathrm{MW}$ and $50 \mathrm{MW}$ plants. The costs are:

Low Million s

High

$\begin{array}{lll}25 \mathrm{MW} & 6.46 & 9.70\end{array}$

$50 \mathrm{MW} \quad 10.34 \quad 1551$ 
A $20 \%$ cost contingency is added to the plant/wellfield surface facilities, development costs and well drilling costs to cover unexpected costs and cost overruns. Using this contingency level is justified since it agrees with the difference between actual completed plant costs shown in Table 4 and the CENTPLANT generated estimates. Geothermal experts who have studied geothermal development costs agreed that $20 \%$ is probably the minimum contingency, while $30 \%$ may be more appropriate for a project as ambitious as the Hawaii project ${ }^{3}$

The CENTPLANT generated estimate for dollars per gross kilowatt hour which NEA uses for a 25 net MW (275 gross MW) plant is $20 \%$ below the average actual cost per gross kilowatt hour of plants constructed in the 20 to $30 \mathrm{MW}$ range. The DAHI report estimate is $52 \%$ below the average. For dollars per net kilowatt hour, the percentages are NEA 22\% below and DAHI 55\% below.

Only one of the three plants listed, shows a construction time for building a geothermal power plant. The figure is higher than estimates of Stone \& Webster Engineering Company of Denver, Colorado who estimate 12 months minimum construction time, and 24 months as an average. ${ }^{2}$ NEA uses the estimate of 12 months, while the DAHI report estimates 7 months or less construction time per plant.

Table 5 shows the geothermal power plant capitai sosts estimated by :VA using the CENTPLANT program. See Appendix $B$ for more detail on the CENTPLANT output.

A comparison of NEA and DAHI total development costs for twenty 25 net MW power plants and their required wells is shown in Table 6.

1/ Alex Sifford, Geothermal Program Manager, Resource Development Division, Oregon Department of Energy.

21 Alex Sifford, Geothermal Program Manager, Resource Development Division, Oregon Department of Energy. 
Table 4

\section{GEOTHERMAL PLANT COST COMPARISON}

\begin{tabular}{|c|c|c|c|c|c|c|c|c|c|c|}
\hline \multirow{2}{*}{$\begin{array}{lll} & \vdots \\
& \end{array}$} & \multirow[b]{2}{*}{ Year } & \multirow{2}{*}{$\begin{array}{l}\text { Gross } \\
\text { Mega- } \\
\text { watts }\end{array}$} & \multirow{2}{*}{$\begin{array}{l}\text { Net } \\
\text { Mega- } \\
\text { watts }\end{array}$} & \multicolumn{3}{|c|}{ Year Buili } & \multirow{2}{*}{$\begin{array}{l}\text { Cost } \\
\text { sM } \\
19905\end{array}$} & \multicolumn{2}{|c|}{1990} & \multirow{2}{*}{$\begin{array}{l}\text { Mouths } \\
\text { Const } \\
\text { Time }\end{array}$} \\
\hline & & & & $\begin{array}{l}\text { Cost } \\
\text { SM }\end{array}$ & $\begin{array}{l}\text { S/GR } \\
\text { KWW }\end{array}$ & $\begin{array}{l}\text { STNet } \\
\mathbf{K W}\end{array}$ & & $\begin{array}{l}\text { SWGR } \\
\mathbf{K W}\end{array}$ & $\begin{array}{l}\text { SWNet } \\
\mathbf{K W}\end{array}$ & \\
\hline $\begin{array}{l}\text { Plants in } y \\
20-30 \mathrm{MW} \text { Range }\end{array}$ & & & & & & & & & & \\
\hline Bear Canyon & 1989 & 220 & 20.0 & 348 & 1582 & 1740 & 353 & 1605 & 1765 & \\
\hline Rooseveit & 1984 & 235 & 200 & 36.0 & 1532 & 1800 & 392 & 1668 & 1960 & 28 \\
\hline West FordFlat & 1989 & 29.7 & 270 & 47.0 & 1582 & 1741 & 47.7 & 1605 & 1766 & \\
\hline $\begin{array}{l}\text { Plants in } \\
20-30 \mathrm{MW} \text { Raoge }\end{array}$ & & 752 & 670 & 1178 & & & 1222 & 1625 & 1823 & \\
\hline $\begin{array}{l}\text { (2SMW) NEA } \\
\text { LOW }\end{array}$ & 1990 & 275 & 250 & & & & 373 & 1357 & 1493 & 12 \\
\hline $\begin{array}{l}\text { (2SMW) NEA } \\
\text { High }\end{array}$ & 1990 & 275 & 25.0 & & & & 40.9 & 1488 & $: 6.6$ & 12 \\
\hline (2SMW) DAHI & 1990 & 275 & 250 & & & & $=9.4$ & 068 & $\therefore 74$ & $:$ \\
\hline $\begin{array}{l}\text { Perceat Below } \\
\text { Average }\end{array}$ & & & & & & & & & & \\
\hline $\begin{array}{l}\text { (2SMW) NEA } \\
\text { LOW }\end{array}$ & & & & & & & & $20 \%$ & $22 \%$ & \\
\hline $\begin{array}{l}\text { (25) NEA } \\
\text { High }\end{array}$ & & & & & & & & $9 \%$ & $11 \%$ & \\
\hline (2SMW) DAFI & & & & & & & & $52 \%$ & $55 \%$ & \\
\hline
\end{tabular}

y Nex Sifford, Oregon Departreet of Energy, in the report Taoovative Design of New Generating Plants," by Gordon Bloomqurs, John Geyer, and Alex Sifford, produced by the Washington State Energy office for the Bonaeville Power Adrainistration, July 1989.

Note: Construetion costs were brought up to 1990 dollars using an annual construction cost inflation rate of $143 \%$. This rate is based on Hasdy-Whitman public utility indexes for eleetric light and power plant construeticn. Sce earlier footnote.

Table 5

GEOTHERMAL POWER PLANT CAPITAL COST (1990 dollars) Smillion

\begin{tabular}{ccc}
\hline $\begin{array}{l}\text { Plant Size } \\
\text { (Megawatts) }\end{array}$ & $\begin{array}{l}\text { Low } \\
\text { Estimate }\end{array}$ & $\begin{array}{l}\text { High } \\
\text { Estimate }\end{array}$ \\
\hline \hline & & \\
25 & 107.79 & 129.40 \\
0 & 197.07 & 29.10 \\
\hline \hline
\end{tabular}

Note: Includes initial wells, wellfield surface facilities, power plant. transmission lines, resource assessment, permits, licenses, $20 \%$ cost contingency. Does not include replacement wells. 
Table 6

COST COMPARISON (MS)

PLANTS AND WELLS

25 Net MW Plants

Cost Comparison (MS)
Plant/Wellfield

Surface Facilities
Wells

$\begin{array}{ll}600.0 & 12622 \\ 600.0 & 1300.9 \\ 675.0 & 1659.6 \\ 900.0 & 2004.6\end{array}$

720.0

$\$ 10.0$

$=080.0$

Total

Without Contingency:

DAHI 19865

NEA 1990 s (high estimate)

With 20\% Contingency:

NEA 1990 s (low estimate

VEA 1990 s (high estimate)

8411

1345.9

1507.9

6622

984.6

1104.6

8411

1345.9

1507.9

NEA 1990 s (high estimate)

Note: The 1986 DAHI cost figures ate brought up to 1990 levels using inflation rates calculated from indexes found in the Statistical Abstract of The United States, 109th Edition, U.S. Dept. of Commerce. The annual construction cost inflation rate used for plant/well field surface facilities is $143 \%$ based on Handy-Whitman public utility indexes for electric light and power plant construction. The annual manufacturing cost inflation rate for the cable portion of the project is $183 \%$ based on the producer price indexes for machinery and equipment.

\section{TRANSMISSION CABLE SYSTEM}

\section{Submarine Transmission Cables}

The submarine cable to be used in this project must be able to withstand the rigors of the underwater environment. High pressures at deep depths, tidal flows, currents of uncertain direction and force, steep and difficult terrain, and the corrosive nature of sea water all combine to make the design and construction of a reliable cable system a formidable task. However, there are in operation fully functional and reliable submarine cable systems around the world, and although the Hawaii Cable Project proposes to install its cable system at deeper depths than 
any of the others, there is little doubt that the design and engineering can be accomplished. The question, is at what cost?

Table 7 gives a basic description of the type of cable thought to be needed for the cable project, and Figure 3 shows its design characteristics in cross section. This specially designed oil containing, pressurized cable would be used for the 42 mile length between Hawaii and Maui (see Table 1, Segments $4 H, 1 A, 1 M$ ).

The 96 mile length from Maui to Oahu (Table 1, Segment 3M), in shallower less treacherous water, will use a combination of solid cable with single and double armored lengths. This cable scenario is in agreement with that proposed by the Pirelli Cable Corporation and found in the DAHI report.

Notes on the cable calculation:

1. The costs of cable manufacturing are from Pirelli Cable Corporation as found in the DAHI report. The cost is in 1986 dollars. These costs are brought up to 1990 dollars using a 183\% per year inflation factor. See Table 3, footnote 2 .

2. The cost of oil pressurization stations is from Pirelli Cable Corporation as found in the DAHI report. The costs are in 1986 dollars. These costs are brought up to 1990 dollars using a $183 \%$ per year inflation factor. See Table 3, footnote 2 .

3. An allowance for slope and bend for the undersea distances is $20 \%$ for the Hawaii-Maui segment and $5 \%$ for the Maui-Uahu segment.

4. Cost of the cable laying vessel, which will have to be constructed, is from William Bonnet, Hawaii Deep Water Cable Program Manager, in a letter to Nelson Ho, March 10, 1987. The 1987 figure is brought up to 1990 dollars using a $228 \%$ per year inflation factor. (U.S. Bureau of Labor Statistics, Producer Price Index for transportation equipment).

5. We assumed two helper vessels will be required to assist in laying the cable. We base this assumption on an article appearing in the Geothermal Resources Council Bulletin, December 1989, page 20 which described the deep-water cable test. Besides the cable laying vessel itself, the mother ship of the submersible vessel used to guide and examine the cable. and a monitoring and evaluation vessel to kecp track of ocean currents is required in the uperation.

6. The cable laying costs and timetable are approximations from the DAHI report. 
Table 7

\section{GENERAL DESCRIPTION OF THE PREFERRED CABLE}

The cable that is currently being evaluated is a self-contained oil-filled (SCOF) cable. with a $300 \mathrm{kVdc}$ voltage, capable of a total transmission load of 250 megawatts (MW) oi electric energy. 1 it is 4.7 inches (1195 mm) in diameter and weighs $24.46 \mathrm{lbs}$. per foot ( $36.4 \mathrm{~kg}$ per meter) in air and $1734 \mathrm{lbs}$ per foot $(258 \mathrm{~kg}$ per meter) in water. The current estimated cost of manufacturing the cable is approximately $\$ 280$ per yard ( $\$ 93.33$ per foot) of cable (\$306 per meter).

Selected Basic Design Characteristics of Cable Design No. 116

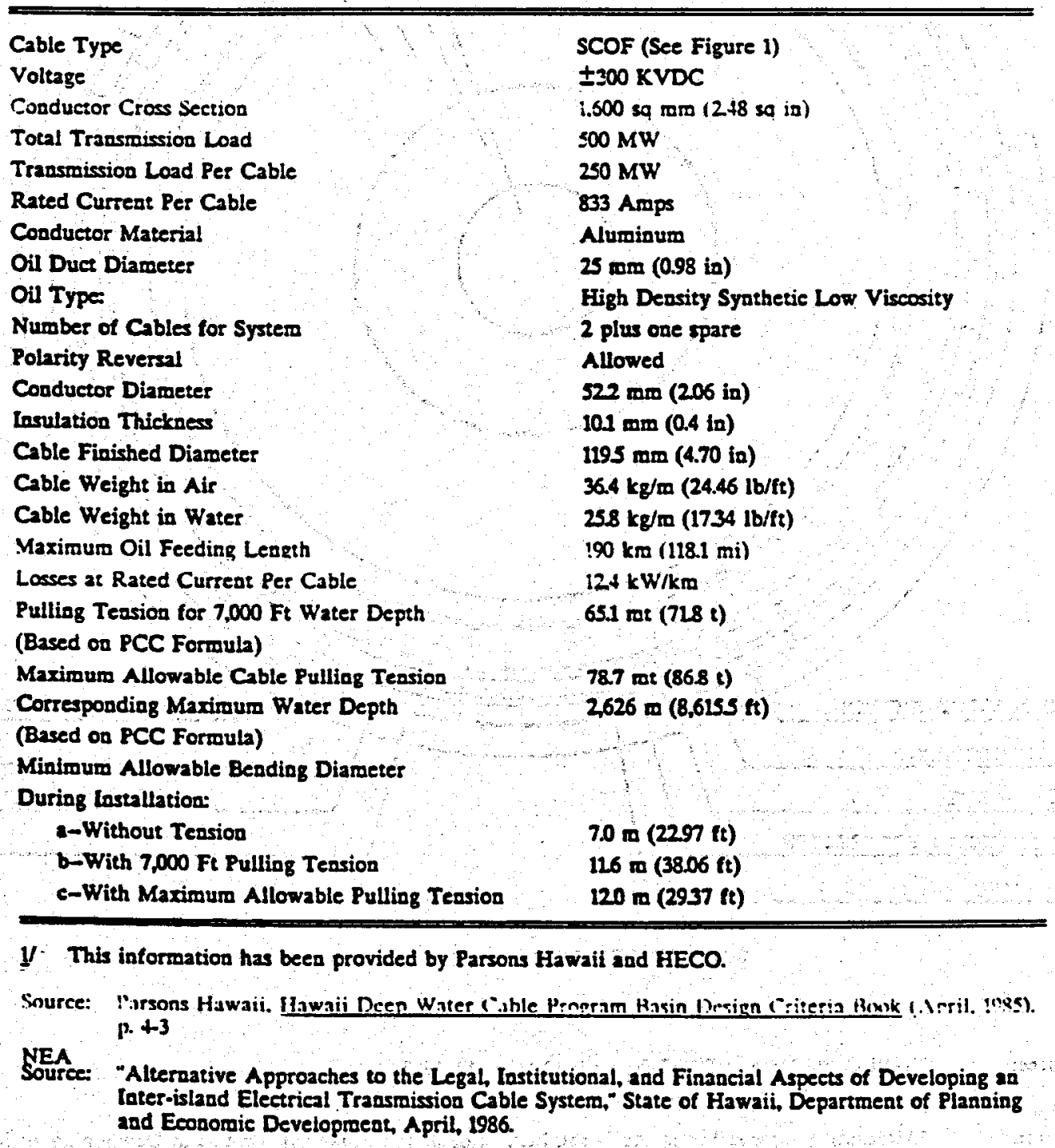


Figure 3

TYPICAL SCOF CABLE CROSS SECTION (Dimensions Based on Cable Design Case No. 110)

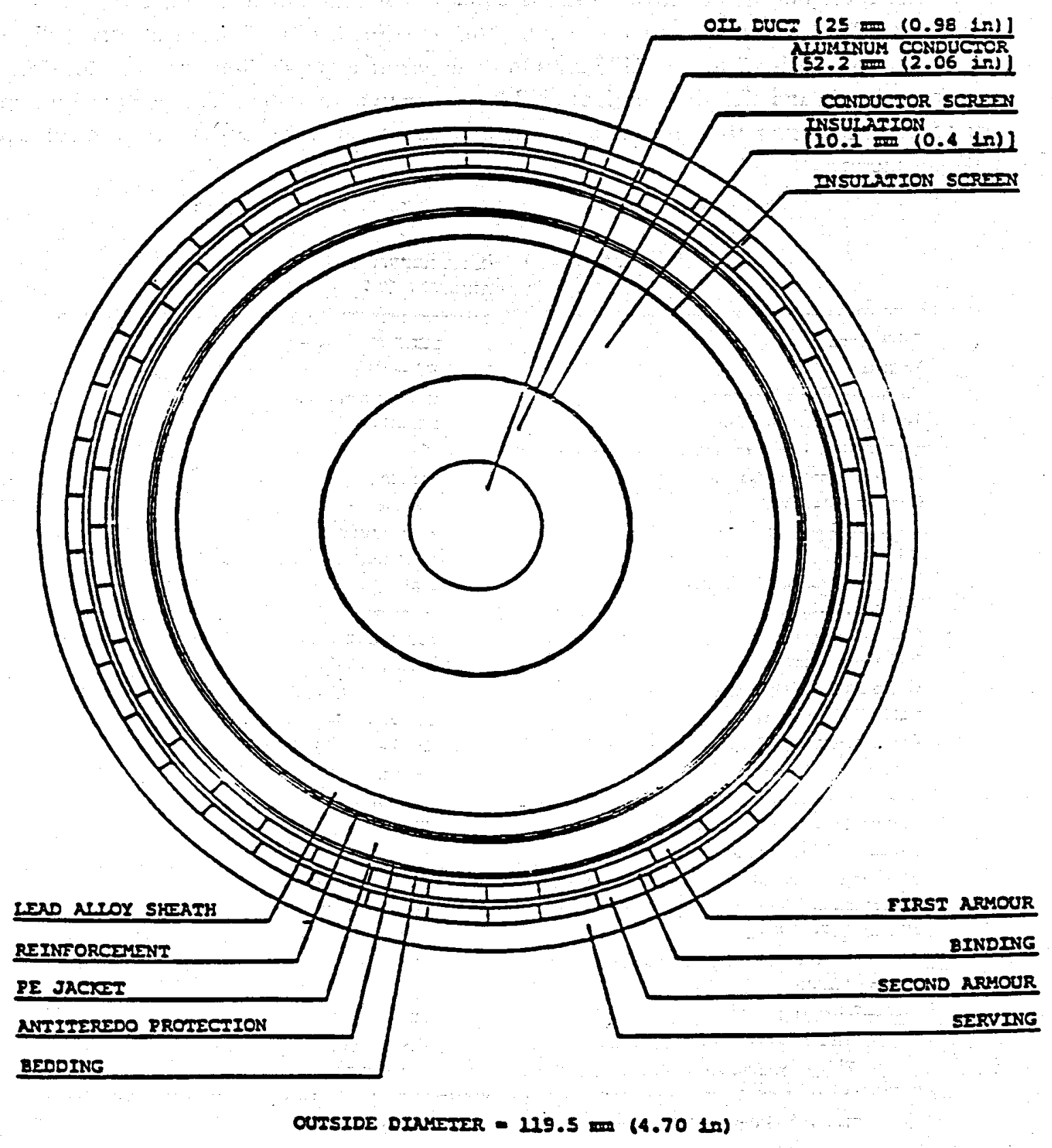

Source: Pirelli Cable Corp, HDWC Program, October 12, 1983

NEA

Source: -Alternative Approaches to the Legal, Institutional, and Financial Aspects of Developing an Inter-island Electrical Transmission Cable System," State of Kawaii, Departinent of Planning and Econornic Developmens, April, 1986. 
CABLE MANUFACTURING COST:

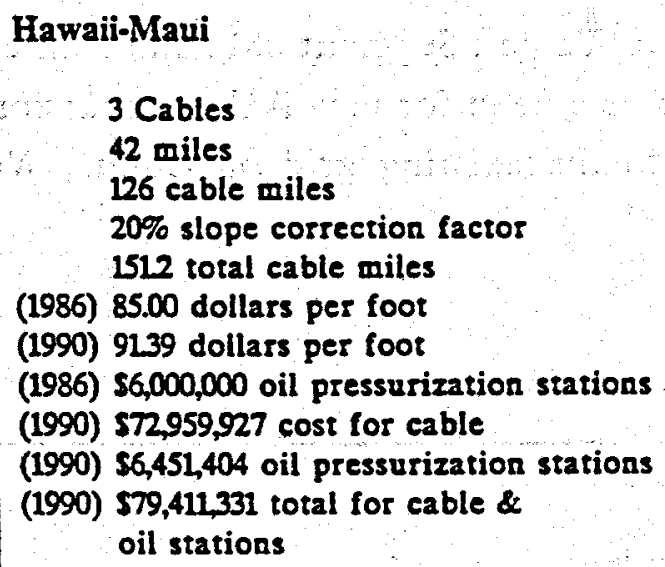

Maui-Oahu

\section{3 cables}

96 miles

288 cable miles

5\% slope \& bend correction factor 302.4 total cable miles

(1986) 8125 dollars per foot

(1990) 8736 dollars per foot

(1990) \$139,485,266 cost for cable

Total Hawaii-Maui-Oahu with

20\% Cost Contingency Factor

(1990) \$262,675,916 Total Cost For Both Cables

\section{CABLE LAYING COST:}

Vessel Construction

(1987) $\$ 17,600,000$

(1990) $\$ 18,831,496$

Vessel Operation Costs Per Day.

$\$ 40,000$ Main Vessel (1990)

\$15,000 Helper Vessel (1990)

Hawaii-Maui

3 Cables

42 miles

126 cable miles

$20 \%$ slope \& bend correction factor

1512 total cable miles

033 cable miles per day

458 days to lay cable

\$18,327,273 Cost of Main Vessel \$13,745,455 Cost of Helper Vessels (2)

\section{Maui-Oahu}

i cables

96 miles

288 cable miles

5\% slope \& bend correction factor

3024 total cable miles

OSO cable miles per day

605 days to lay cable

\$24,192,000 Cost of Main Vessel $\$ 18,144,000$ Cost of Helper Vessels (2)

291 Years to lay cable

Total Hawail-Maui-Oahu with

20\% Cust Cuntingency cust

(1990) S111888,268 Total Cost To Lay Both Cables

(1990) \$374,564,184 Total Cost Manufacture, Delivery, and

Laying of Submarine Cable and Facilities 
Overhead Transmission Lines and Facilities

There will be 108 miles of DC (direct current) transmission line on Hawaii (Table 1, Segments $1 \mathrm{H}, 2 \mathrm{H}, 3 \mathrm{H}$ ), 20 miles on Maui (Table 1 Segment 2M), and 3 miies on Oahu (Table 1, Segment 10). There will also be a need for new AC transmission lines to connect from the converter stations to the existing grid on Oahu. We estimate this length at 15 miles.

Overhead Transmission Lines and Facilities:

\begin{tabular}{|c|c|}
\hline $\begin{array}{r}500,000 \\
\$ 180 \\
\$ 90,000,000 \\
\$ 2 \\
\$ 180,000,000\end{array}$ & $\begin{array}{l}\text { KW } \\
\text { Cost Per Kilowatt } \\
\text { Cost For Termination Facilities } \\
\text { and AC/DC Conversion Stations } \\
\text { Stations } \\
\text { Total Cost For Termination Facilities } \\
\text { and AC/DC Conversion Stations }\end{array}$ \\
\hline $\begin{array}{r}\$ 240,000 \\
108 \\
20 \\
3 \\
2 \\
262 \\
\$ 62,880,000\end{array}$ & $\begin{array}{l}\text { Cost/mile Overhead DC Transmission Lines } \\
\text { Miles Hawaii } \\
\text { Miles Maui } \\
\text { Miles Oahu } \\
\text { Lines } \\
\text { Total Miles } \\
\text { Total Cost Overhead DC Transmission Lines }\end{array}$ \\
\hline $\begin{array}{r}\$ 360,000 \\
15 \\
\$ 5,400,000 \\
20 \% \\
\$ 1,000,000\end{array}$ & $\begin{array}{l}\text { Cost/mile Overhead AC Transmission Lines } \\
\text { Miles Hawaii } \\
\text { Total Cost Overhead DC Transmission Lines } \\
\text { Cost Contingency Factor } \\
\text { Environmental Impact Statement }\end{array}$ \\
\hline
\end{tabular}

(1990) $\$ 298,936,000$ Total Cost For Overhead Transmission Lines and Facilities with $20 \%$ Cost Contingency

Notes on the transmission facilities calculation:

1. The cost of $A C$ and $D C$ transmission lines is from Bonneville Power Administration (BP. 1 ) engineers in Portland. Oregon. The cest it: AC transmission lines is more than for DC transmission lines because AC transmission requires three cables instead of two as is the case for DC. This third cable requires that the towers be larger and the rightof-way wider for $A C$ than for $D C$. 
2. Two $A C / D C$ conversion stations and termination facilities will be required for the project. One will be located at the source of power generation on Hawaii and the other on Oahu where the DC power lines join the AC power distribution grid. BPA engineers estimate that a termination and conversion facility for a 500 MW system using solid state converters and state of the art switching gears and transformers will cost between $\$ 80$ million and $\$ 100$ million. (B.C. Hydro engineers estimate the cost at closer to $\$ 125$ million per station.)

A comparison of NEA and DAHI total costs for submarine and overland power transmission cables and facilities is shown in Table 8:

Table 8

COST COMPARISON (MS)

TRANSMISSION CABLES AND FACILITIES

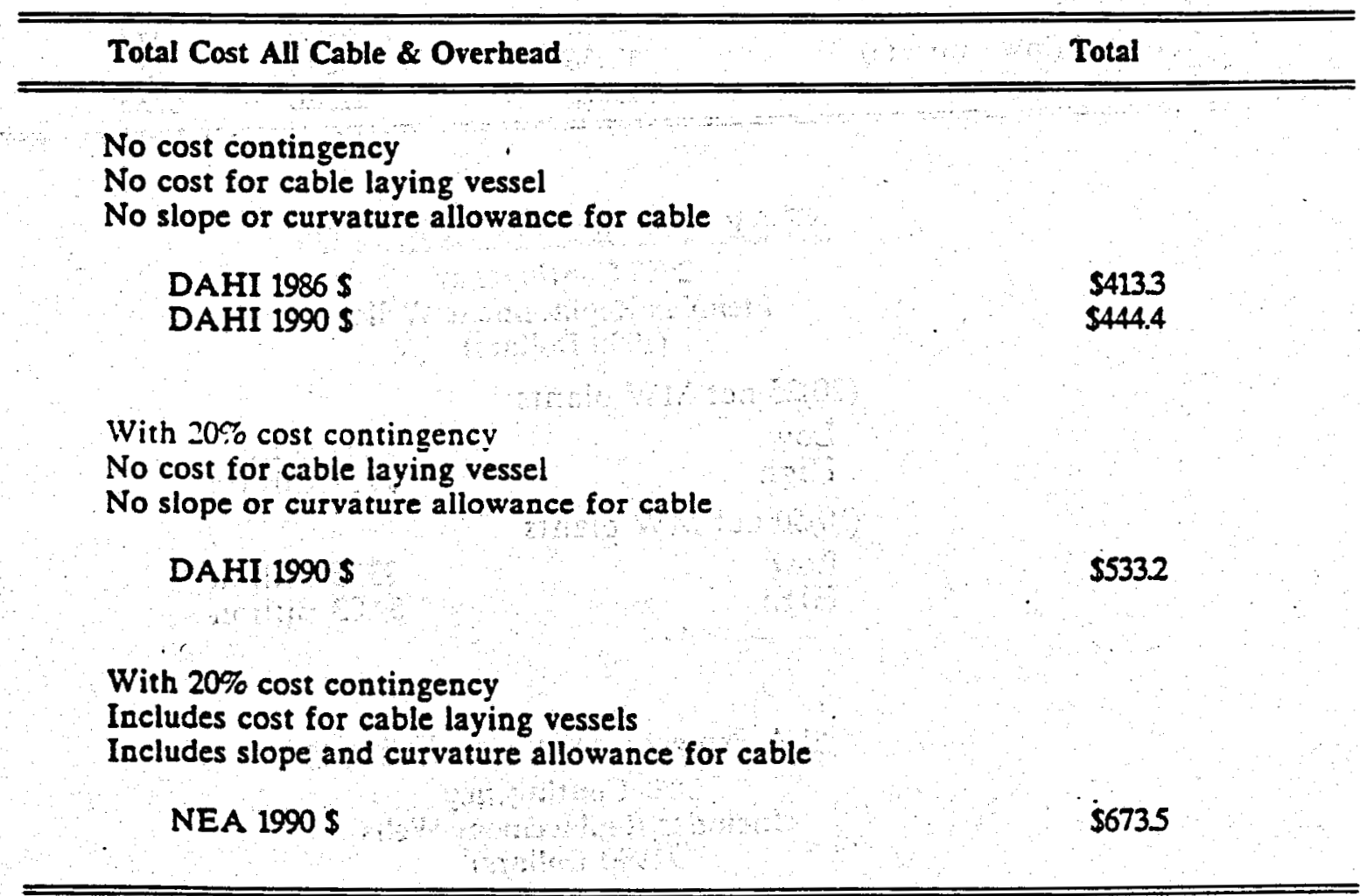

A comparison of NEA and DAHI total project development costs for twenty 25 net MW plants is shown in Table 9. 
Table 9

PROJECT DEVELOPMENT COST COMPARISON

\begin{tabular}{|c|c|c|c|c|}
\hline $\begin{array}{l}25 \text { Net MW Plants } \\
\text { Cost Comparison (MS) }\end{array}$ & $\begin{array}{l}\text { Plant/Wellfield } \\
\text { Surface Facilities }\end{array}$ & Wells & Cables & $\begin{array}{l}\text { Total } \\
\text { Cost }\end{array}$ \\
\hline \multicolumn{5}{|l|}{ Without Contingency: } \\
\hline $\begin{array}{l}\text { DAHI } 1986 \$ \\
\text { DAHI } 1990 \$ \\
\text { NEA } 1990 \$ \text { (low estimate) } \\
\text { NEA } 1990 \text { S (high estimate) }\end{array}$ & $\begin{array}{r}6622 \\
700.9 \\
984.6 \\
1104.6\end{array}$ & $\begin{array}{l}600.0 \\
600.0 \\
675.0 \\
900.0\end{array}$ & $\begin{array}{l}413.3 \\
444.4 \\
561.4 \\
5614\end{array}$ & $\begin{array}{l}1675.5 \\
1745.3 \\
2221.0 \\
2566.0\end{array}$ \\
\hline \multicolumn{5}{|l|}{ With $20 \%$ Contingency: } \\
\hline $\begin{array}{l}\text { DAHI } 1990 \text { \$ } \\
\text { NEA } 1990 \text { (low estimate) } \\
\text { NEA } 1990 \text { (high estimate) }\end{array}$ & $\begin{array}{r}841.1 \\
1345.9 \\
1507.9\end{array}$ & $\begin{array}{r}720.0 \\
810.0 \\
1080.0\end{array}$ & $\begin{array}{l}533.2 \\
673.5 \\
673.5\end{array}$ & $\begin{array}{l}2094.3 \\
2829.4 \\
3261.4\end{array}$ \\
\hline \multicolumn{5}{|c|}{$\begin{array}{l}\text { With } 20 \% \text { Contingency \& Replacement } \\
\text { Wells: }\end{array}$} \\
\hline $\begin{array}{l}\text { DAHI } 1990 \text { \$ } \\
\text { NEA } 1990 \text { (low estimate) } \\
\text { NEA } 1990 \text { S (high estimate) }\end{array}$ & $\begin{array}{r}8411 \\
1345.9 \\
1507.9\end{array}$ & $\begin{array}{l}1440.0 \\
1620.0 \\
2160.0\end{array}$ & $\begin{array}{l}533.2 \\
673.5 \\
673.5\end{array}$ & $\begin{array}{l}2814.3 \\
3639.4 \\
43414\end{array}$ \\
\hline
\end{tabular}

\section{NEA Project Capital Cost Estimate}

20\% Contingency

Includes Replacement Wells

(1990 Dollars)

(20)25 net MW plants

Low

High

(10)50 net MW plants

Low

High
53.64 Billion

$\$ 434$ Billion

$\$ 3.35$ Billion

$\$ 4.03$ Billion

\section{NEA Project Capital Cost Estimate $30 \%$ Contingency \\ Includes Replacement Wells (1990 Dollars)}

(20)25 net IIW nlants

Low

High

$\$ 3.59$ Billion

$\$ 4.65$ Billion

(10)50 net MW plants

Low

High
\$3.56 Billion

$\$ 4.30$ Billion 


\section{NEA - OIL AND SOLAR/OIL POWER PLANT COST ESTIMATES}

\section{OIL PLANTS}

The capital cost for the oil fired power plants used in this analysis is $\$ 800$ per kilowatt in 1990 dollars. This figure was derived from two sources. The 1986 preliminary report from Decision Analysts Hawaii, Inc. uses a figure of $\$ 752$ per kilowatt for its oil fired power plants. This figure is in 1986 dollars. The California Energy Commission in its October 1988 Energy Technology States Report cites the figure $\$ 750$ per kilowatt in 1985 dollars. The average of both these figures in 1986 dollars is $\$ 756$ per kilowatt. Bringing this figure up to 1990 dollars using the $143 \%$ annual inflation factor from the Handy-Whitman public utility index for electric light and power plant construction costs gives us a base capital cost of $\$ 800$ per kilowatt Using this base cost we calculated the total cost per 100 net MW plant as:

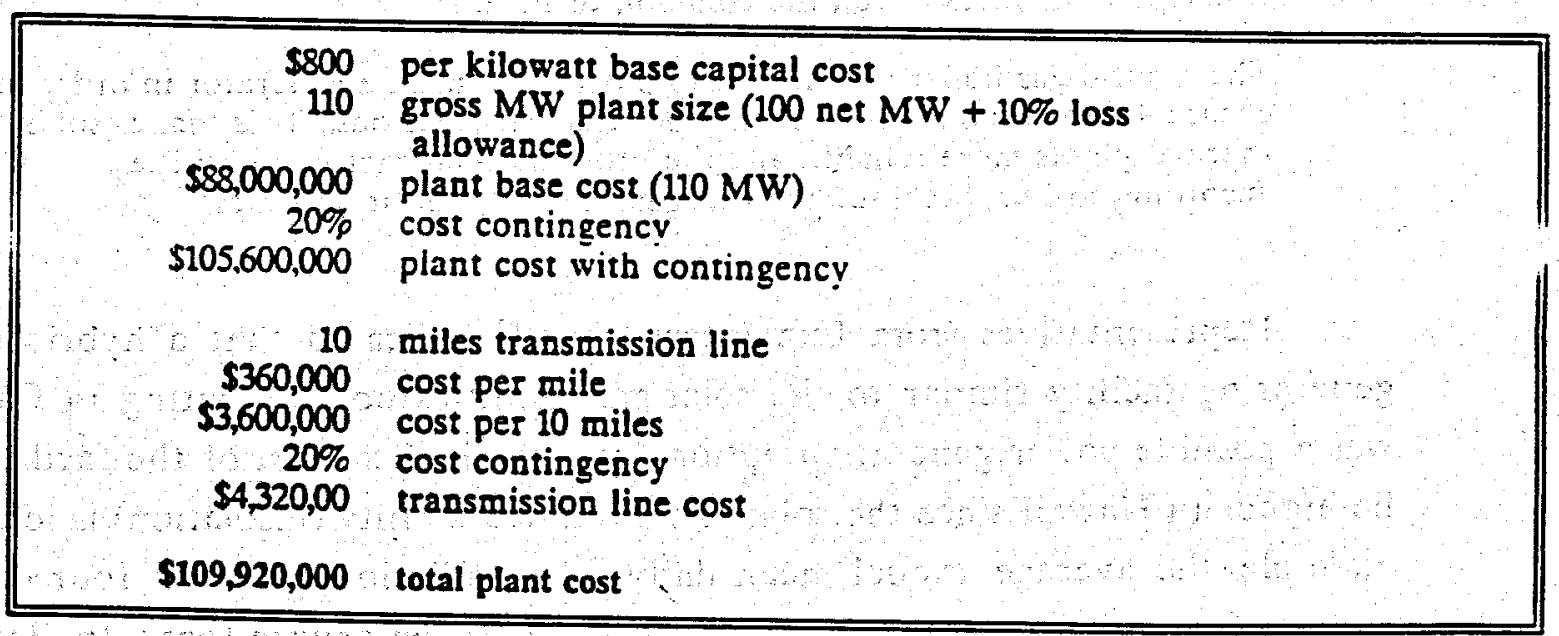

This is the oil plant capital cost used in the analysis. 


\section{SOLAR/OIL PLANTS}

The cost for a solar/oil fired combination (hybrid) power plant comes from Luz International, a builder and developer of solar thermal electr:c power piants. Luz International operates the largest solar facility ever built $(200 \mathrm{MW}$ in the Mohave Desert region of Southern California) and the source of an estimated $90 \%$ of the worlds solar electricity. Luz supplies solar thermal generated electricity to the Southern California Edison power company. Their system is described below: ${ }^{1}$

Luz's Solar Electric Generating Systems (SEGS) use trough mirror assemblies that individually track the sun by way of sophisticated microprocessors and highly precise sun-sensing instruments. The mirrors reflect sunlight onto stainless steel heat collecting pipes covered with a custom-designed absorptive coating.

Inside the pipes, a heat transfer fluid (a synthetic oil) absorbs and transports the thermal energy to a conventional boiler, which converts water to steam. The steam is then superheated with additional solar thermal energy and powers a steam turbine generator connected to the utility's power grid. On cloudy days or during evening hours, steam is generated by a natural gas boiler that runs the same turbine. The system can also operate in a hybrid mode, using both solar thermal heat and natural gas to generate steam from two separate boilers to run the common turbine.

The natural gas boiler is available to power the turbine generator in order to ensure uninterrupted power during peak demand periods. This makes solar therma! plants more reliable, and therefore more attractive to utilities. according to Luz, because they can guarantee power at all times.

Representatives from Luz International ${ }^{2}$ indicated that a hybrid solar/oil generating facility similar to the solar/gas facilities now operating in California was a possible power generation option for Hawaii The cost of the facility would be higher in Hawaii since the solar radiation availability (insolation) is lower. For example, the average annual mean daily solar radiation based on four collection stations in Southern California is about 1850 BTU's per square foot. ${ }^{3}$ In Hawaii the

1/ Power Surge The Status and Near Term Potential of Renewable Energy Technologies. By

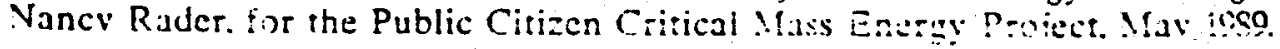

2/ Phone conversation with Howard Hampton, Luz International. LTD. February, 1990.

3/ The solar availability figures (insolation) come from the U.S. Dept. of Energy publication Input Data for Solar Systems, by V. Cinquemani. J.R. Owenby Jr, and R.G. Baldwin, November 1978. 
average annual mean daily solar radiation available, based on four collection stations, is about 1550 BTU's per square foot. Consequently, more solar collector surface area would be required in Hawaii to collect the same amount of availabie energy as in Southern California. Since solar collectors are the major component of the solar facility the cost would increase accordingly. Below is a comparison that shows the estimated difference in cost between a 100 net $\mathrm{MW}$ solar/oil plant built in Southern California based on Luz International cost estimates ${ }^{1}$ and a 100 net MW plant built in Hawaii.

\begin{tabular}{|c|c|}
\hline & $\begin{array}{r}\text { COMPARISON OF SO } \\
\text { CALIFORI }\end{array}$ \\
\hline & California \\
\hline solar costs & 100 net MW California \\
\hline 1,850 & btu/sq. ft insolation \\
\hline $3,413,000$ & btu per $M W$ \\
\hline 1,845 & $\begin{array}{l}\text { sq. ft collector to capture } 1 \\
\text { gross } \mathrm{MW}\end{array}$ \\
\hline $\mathbf{1 1 0}$ & gross MW \\
\hline 202,935 & $\begin{array}{l}\text { sq. ft. collector for } 110 \\
\text { gross } \mathrm{MW}\end{array}$ \\
\hline 0.35 & conversion efficiency \\
\hline 579,815 & $\begin{array}{l}\text { sq. ft collector for } 110 \\
\text { gross } \mathrm{MW}\end{array}$ \\
\hline 1,988 & S/kW for solar collectors \\
\hline 377 & s/for sq. ft. solar collector \\
\hline $218,680,000$ & S for solar collectors \\
\hline 250 & S for common generator \\
\hline 2238 & S kW for solar component \\
\hline 550 & kW for oil component \\
\hline 2,788 & $\$ \mathrm{~kW}$ for solar/oil plant \\
\hline
\end{tabular}

\begin{tabular}{|c|} 
Hawaii \\
solar costs 100 net MW Hawaii \\
1,550 btu/sq. ft insolation \\
$3,413,000$ btu per MW \\
1,845 sq. ft collector to capture 1 \\
gross MW \\
110 gross MW \\
242,213 sq. ft. collector for l10 \\
gross MW \\
0,35 conversion eificiency \\
692,037 sq. ft collector for 110 \\
gross MW \\
2,373 s/kW for solar collectors \\
377 s/for sq. ft. solar collector \\
$261,005,161$ sfor solar collectors \\
250 s for common generator \\
$2,623 \mathrm{~s} k W$ for solar component \\
$550 \mathrm{~kW}$ for oil component \\
3173 s kW for solar/oil plant \\
\end{tabular}

$1 /$ Northwest Power Planning Council. Staff Issue Paper 89-46, Solar Electric Resources, November, 1989. 
Using this base cost we calculated the total cost per $100 \mathrm{MW}$ solar/oil hybrid power plant as:

53173 per kilowatt base capital cost

110 gross $\mathrm{MW}$ plant size $(100$ net $\mathrm{MW}+10 \%$ loss allowance)

\$349,030,000 plant base cost (110 MW)

$20 \%$ cost contingency

$\$ 418,836,000$ plant cost with contingency

10 miles transmission line

$\$ 360,000$ cost per mile

$\$ 3,600,000$ cost per 10 miles

$20 \%$ cost contingency

$\$ 4,320.00$ transmission line cost

$\$ 423,156,000$ total plant cost

This is the solar/oil plant capital cost used in the analysis.

In the analysis it is assumed the solar component of the plant contributes $30 \%$ of the total power output. The oil component provides $70 \%$. 


\title{
NEA - GEOTHERMAL PROJECT HAZARD ASSESSMENT
}

The island of Hawaii is the product of volcanic eruptions that have occurred over millions of years. Eruptive activity is currently occurring and as stated by the authors of a recent U.S.G.S. Publication: ${ }^{1}$

\begin{abstract}
"Similar eruptions have continued into bistorical time on the islands of Hawaii and Maui and undoubtedly will occur in the future, especially on Kilauea and Mauna Loa Volcanoes. Most Hawaiian eruptions form lava flows that endanger chiefly property; explosive eruptions are relatively rare but are more likely to threaten people. As intensive land development expands toward areas of relatively high hazard, the threat to life and property will increase accordingly."
\end{abstract}

The current eruption which began in 1982 and has continued through the present, has covered well over 12,000 acres and caused damages of more than $\$ 11$ million. It is the same eruption which completely covered what was to have been a geothermal development site and prompted a land exchange with the state, insuring geothermal proponents the pursuit of their development objective.

Figure 4 shows the five major volcanoes that make up the island of Hawaii. Of the five, the two most active are Mauna Loa and Kilauea. Tables 10 and 11 list the eruptive activity that has occurred on Mauna Lua and Kilauea Volcanoes in the recent past. Kilauea has recorded 65 eruptions in the last 233 years and has an eruption interval of about 35 years. Mauna Loa has had 37 eruptions in 152 years averaging 4 years between eruptions. Kilauea, currently erupting, is the most active of the two. The type and pattern of its volcanic activity is shown in Figures 5,6 , and 7 .

Areas along the faults and rifts of Kilauea and around other areas of potential volcanic activity have been identified by type and level of hazard (Figures 8 and 9). From information the U.S.G.S. has collected. it has developed a series of hazard zone maps and describes them in the following manner:

11. Volcanism in Hawaii, Chapter 22. U.S. Geological Survey Professional Paper 1350, 1986. 
Figure 4

MAP OF THE ISLAND OF HAWAII, SHOWING THE FIVE MAJOR VOLCANOES THAT MAKE UP THE ISLAND, AND THE HISTORIC LAVA FLOWS.

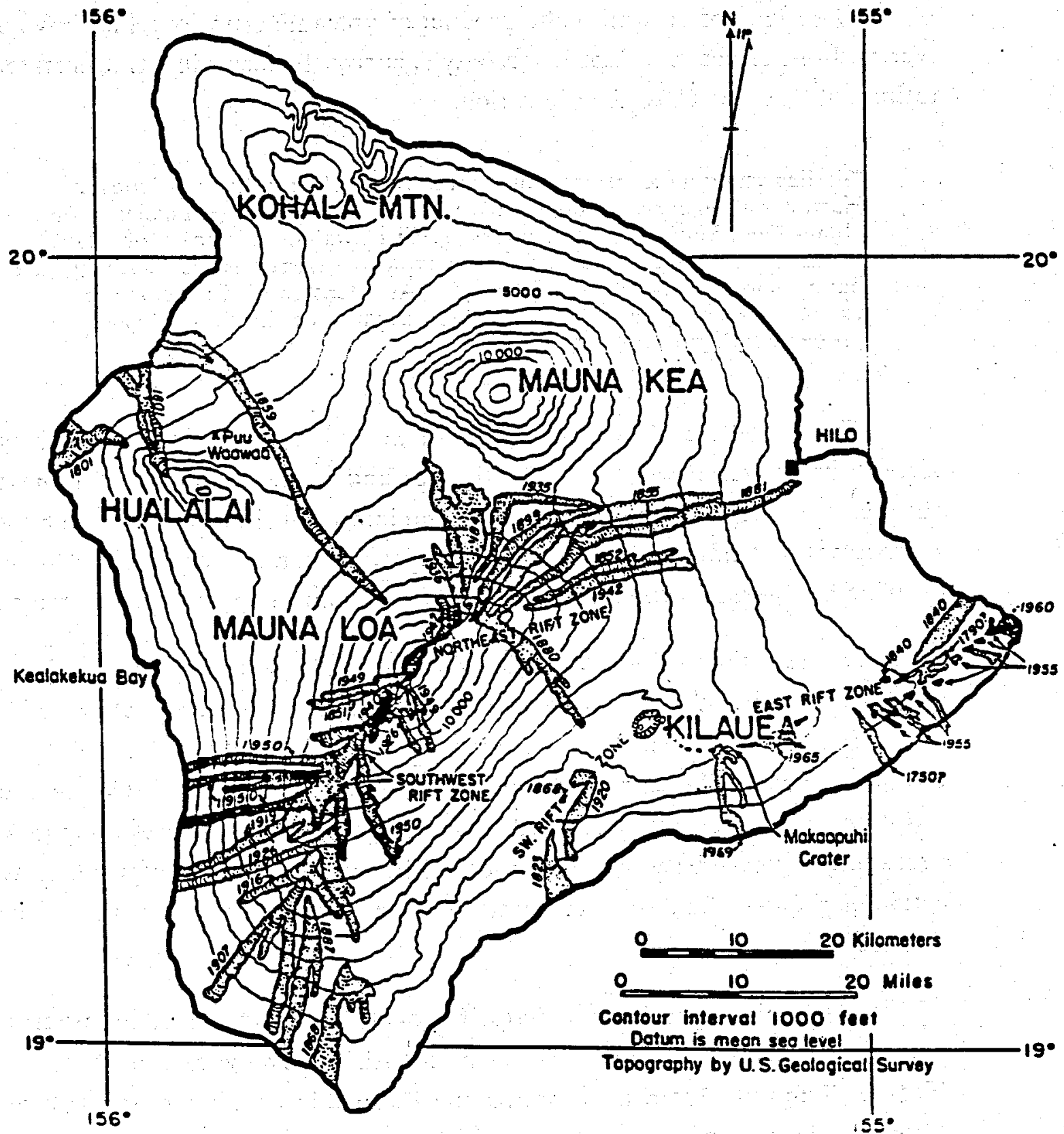

Source: Volcanoes in the Sea, The Geology of Hawaii, Gordon A. Macdonald and Agatin T. Abbott, 1970. 
Table 10

\section{VOLCANISM IN HAWAII \\ Historical Eruptions of Mauna Loa Through May, 1985}

[Daher lool no dua: overy (II quetionable dua]

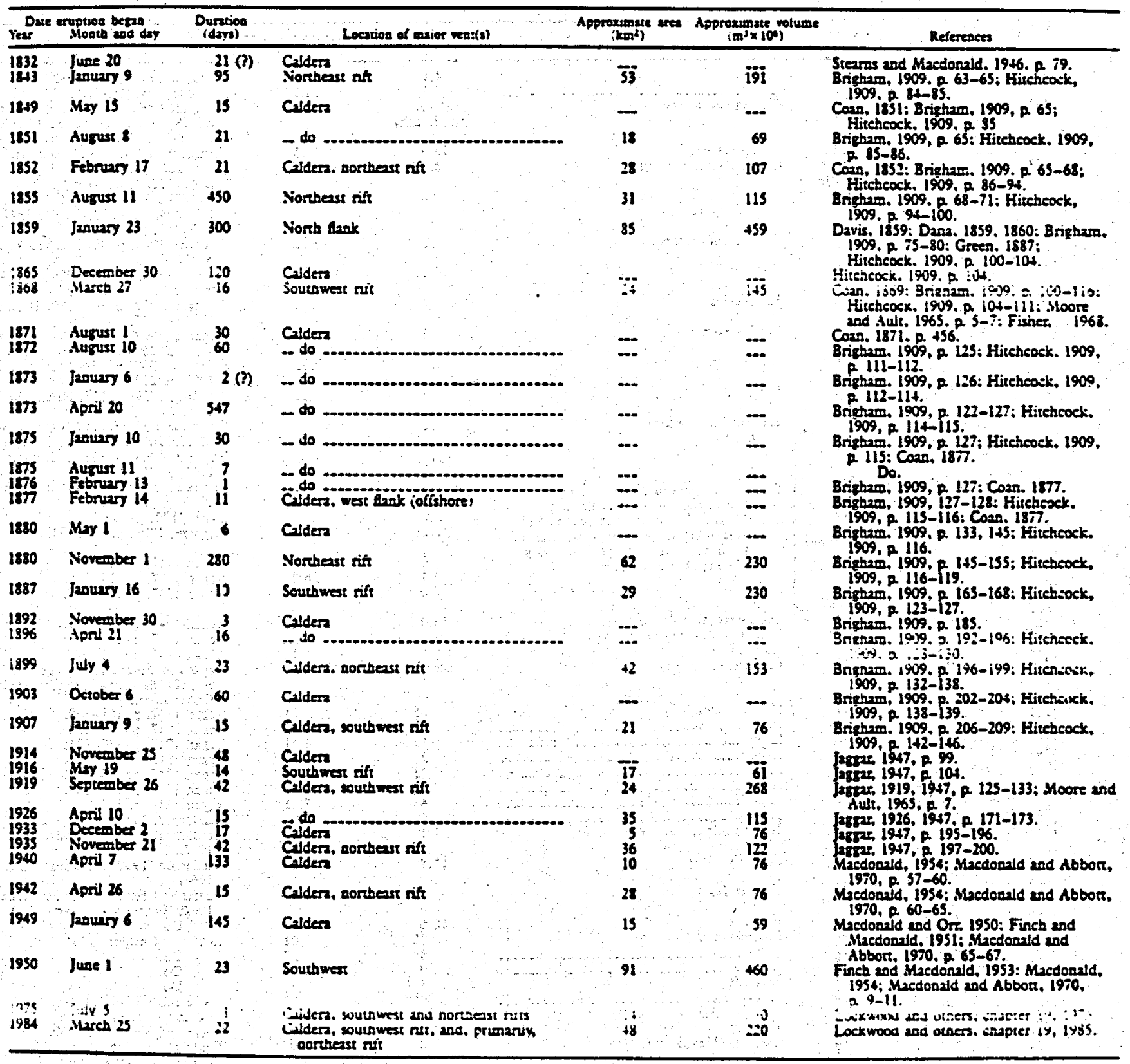

Source: Volcanism In Hawaii U.S. Geological Survey Professional Paper 1350. 
Table 1

VOLCANISM IN HAWAII

Historical Eruptions of Kilauea Through May, 1985

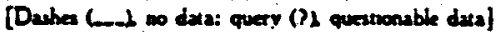

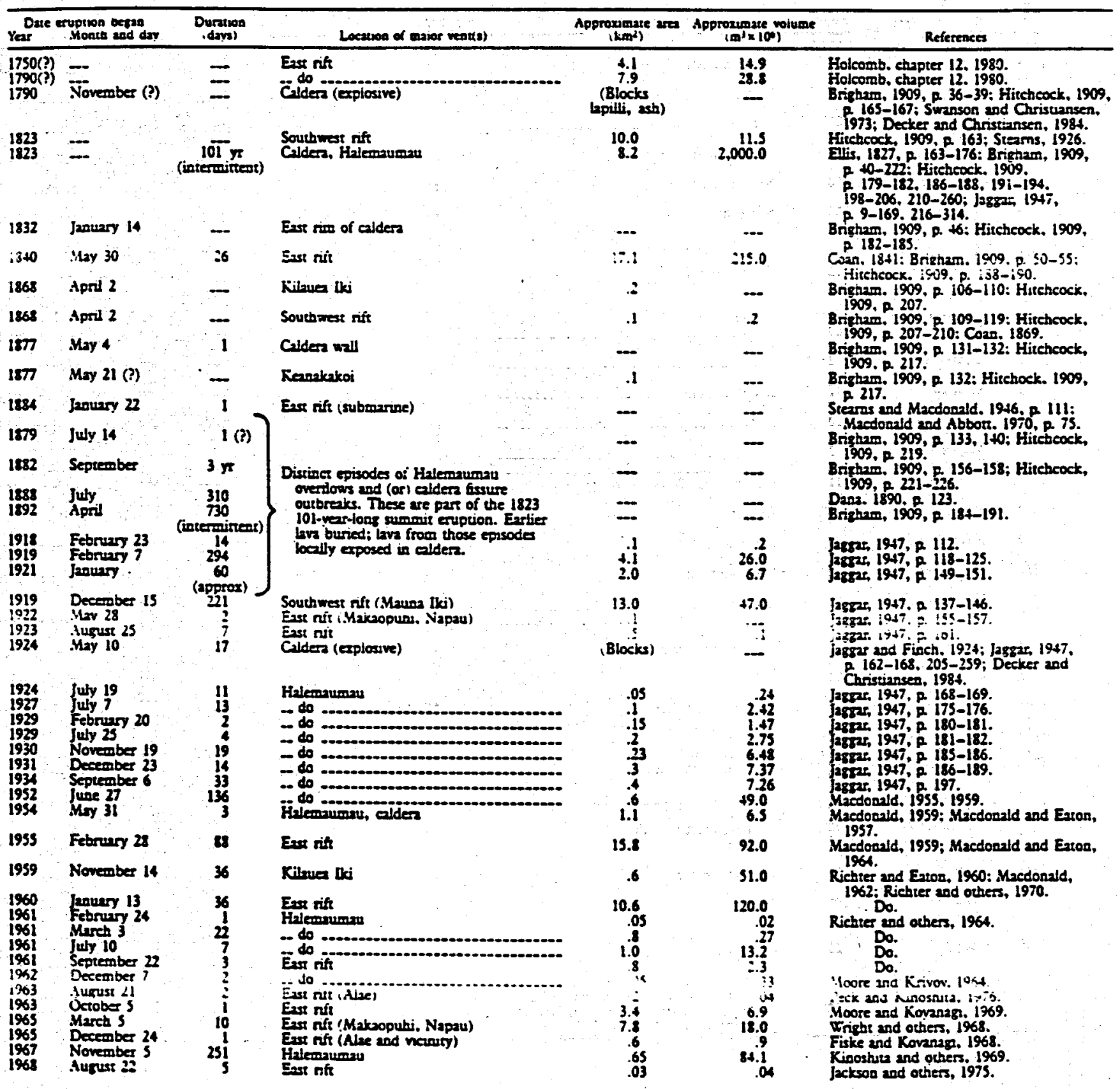


Table 11 Cont'd.

VOLCANISM IN HAWAII

Historical Eruptions of Kilauea Through May, 1985

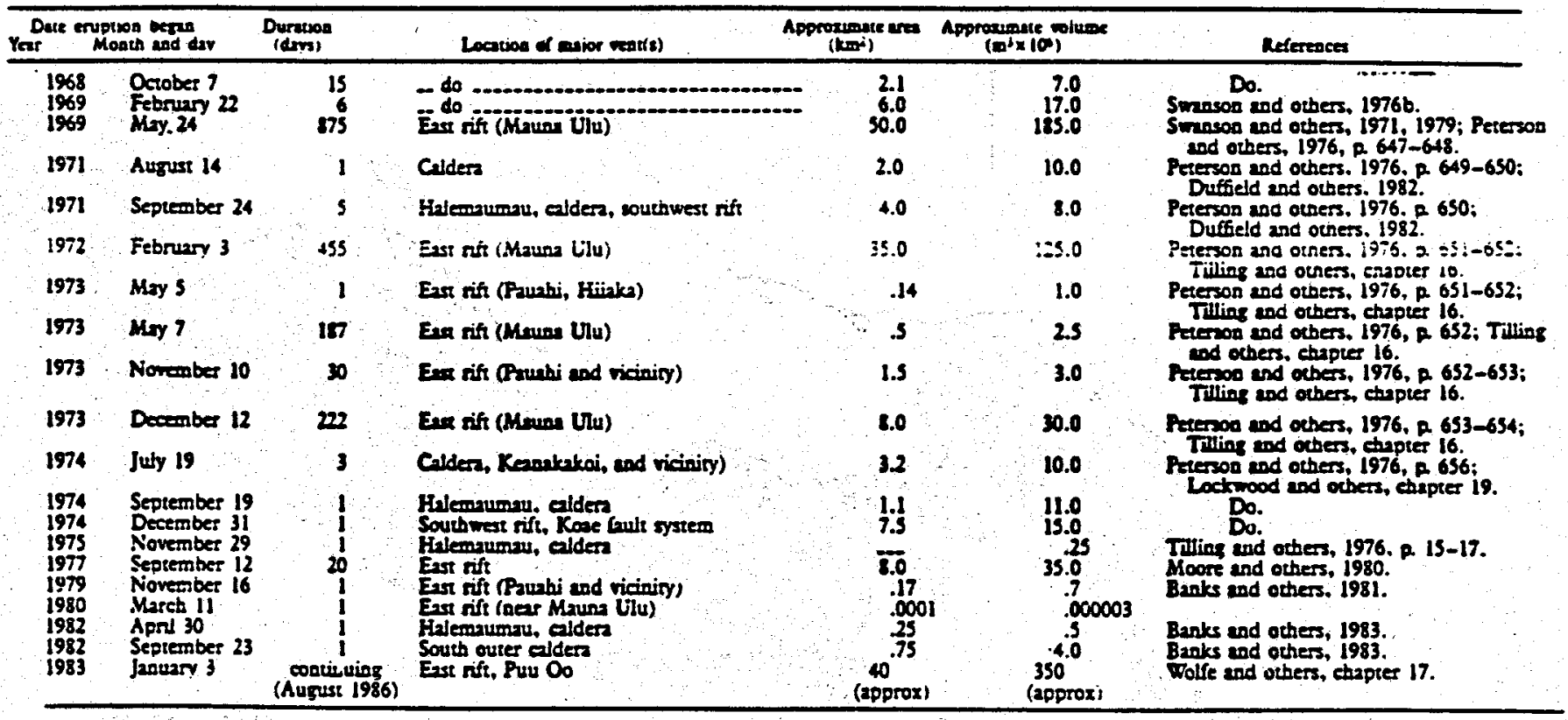

Source: Volcanism In Hawaii U.S. Geological Survey Professional Paper 1350. 
Figure 5

TYPE OF ERUPTIVE BEHAVIOR OF KILAUEA VOLCANO

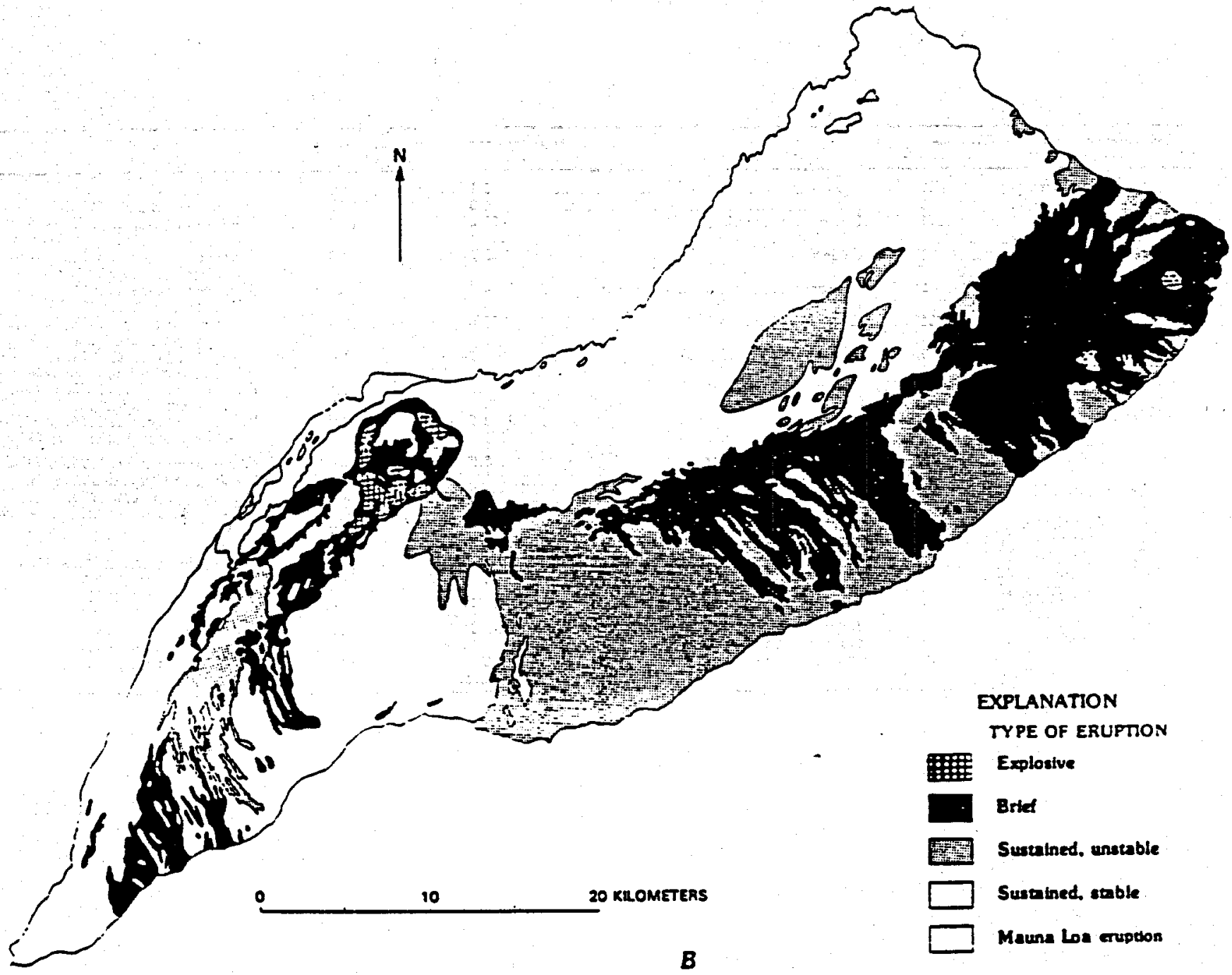

Source: Volcanism_In Hawaii U.S. Geological Survey Professional Paper 1350. 
Figure 6

KILAUEA VOLCANO SHOWTNG THE LOCATION OF

THE SUMMIT CALDERA, TWO ACTIVE RIFT ZONES, THE KOAE AND HIIINA FAULT SYSTEMS, AND

THE LOCATION OF HISTORIC ERUPTIONS ON THE RIFT ZONES.

\section{THE SUMMIT CALDERA FLOOR IS COVERED BY LAVAS ERUPTED BETWEEN 1885 AND 1982.}

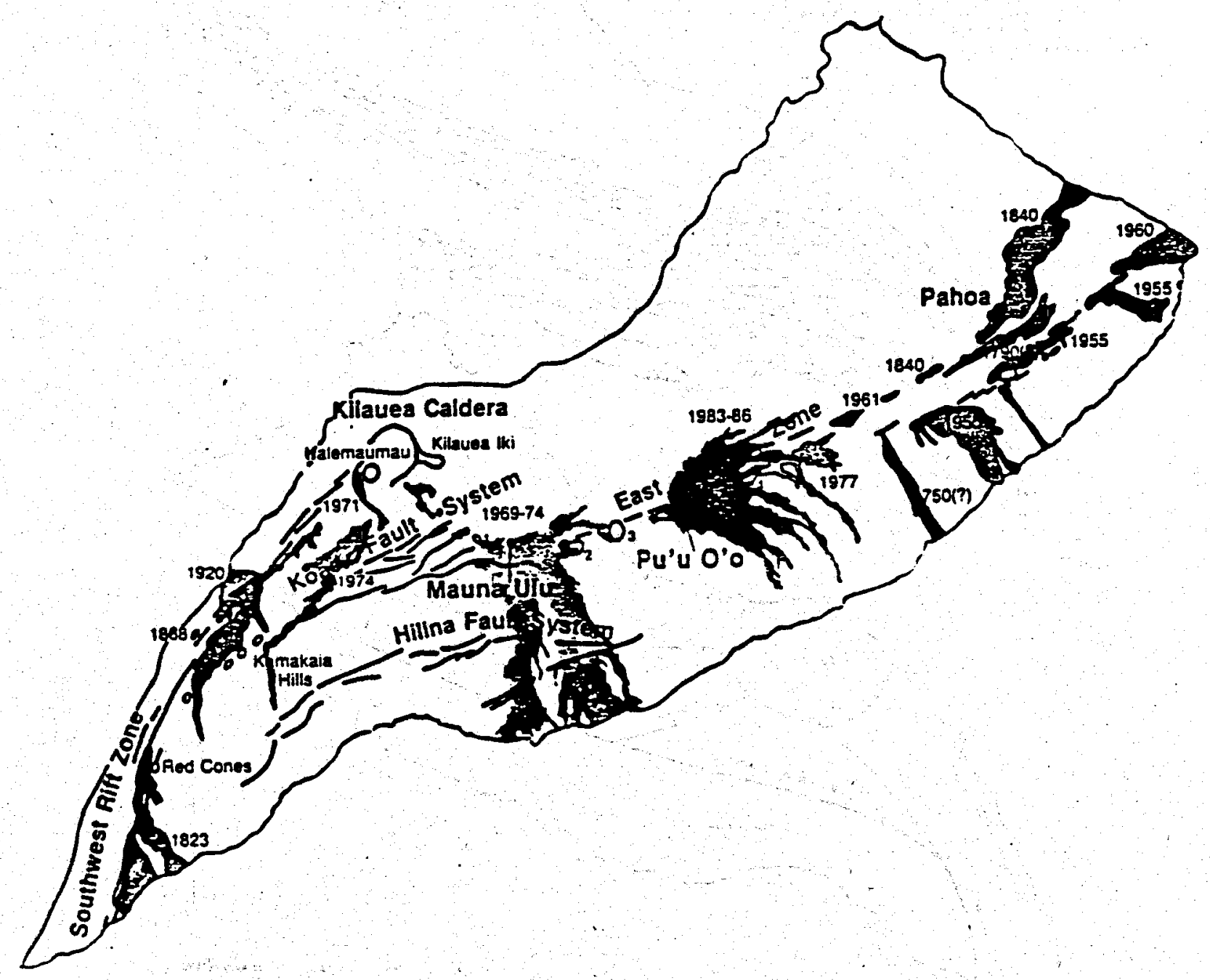

Source: Earthquakes and Volcanoes, U.S. Geological Survey, (formerly Earthquake Information Bulletin) Vol. 18, No. 1 1986. 
Figure 7

\section{ERUPTIVE HISTORY AND LONG-TERM BEHAVIOR OF KILAUEA VOLCANO}

\section{Structure of Kilauea}

A. Mapped structures, simplified from Holcomb (1980b).

B. Structural subdivisions, modified from Swanson and others (1976a).

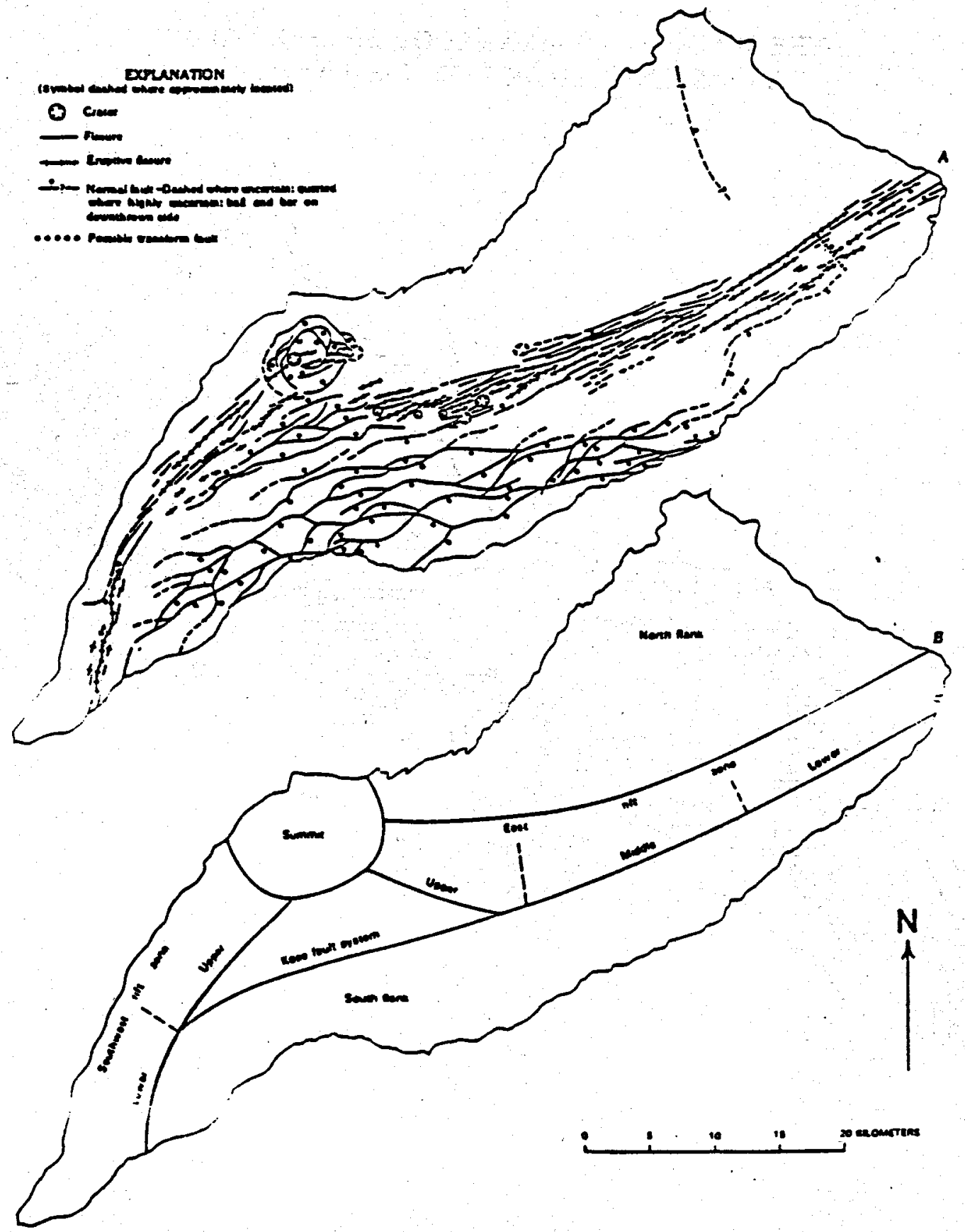

Source: Volcanism In Hawaii U.S. Geological Survey Professional Paper 1350. 
The hazard-zone maps distinguish areas in which the general level of hazard is different from that of adjacent areas. However, the level of hazard can vary considerably within any hazard zone, either gradually or abruptly. Direct volcanic hazards. for example. decrease in magnitude gradually across zones away from active vents. For such hazards as lava flows, the frequency with which a specific site is affected decreases with increasing distance; for other hazards such as tephra and gases, the severity of effects diminishes gradually with increasing distance. Such gradational changes in the hazard may extend across an entire zone.

Hazard zones are based chiefly on the assumption that future eruptions will be like those in the past that are known from oral and written histories and from geologic investigations. Some kinds and scales of eruptive events could occur that are not foreseen by these hazards assessments."

According to Dr. Richard Moore of the U.S.G.S. Hawaiian Volcano Observatory, the two most critical hazaros to geothermal development are lava flows and ground subsidence. Dr. Moore believes that earthquakes are also a hazard but that structures can be built to withstand them. However, he notes that surface pipe systems would be in danger and could be easily ruptured.

The area in which the proposed geothermal development is to be located is shown in Figure 8. According to U.S.G.S. studies, this is an area of high risk due to volcanic lava flows. The cross-hatching shows the general area in which the power plants will te located, and the line crossing the island shows the preferred route of the proposed power transmission lines.

The U.S.G.S report cited earlier explains its lava flow hazard zone maps in the following way:

"Hazard zones for lava flows are based chiefly on lava-flow coverage of different areas during specific time periods. The zones are also based partly on the current structural conditions within the volcanoes, on fault scarps and other topographic features that would limit the distribution of lava flows, and on the frequency of past eruptive events."

The greatest degree of hazard exists in Zone 1 and decreases as the zone number increases. Zones 1 and 2 are the zones ui greatest oncern.

1 Conversation with Dr. Richard Moore on April \& 1987. 
Figure 8

\section{HAZARD ZONES FOR LAVA FLOW ON THE ISLAND OF HAWAI}
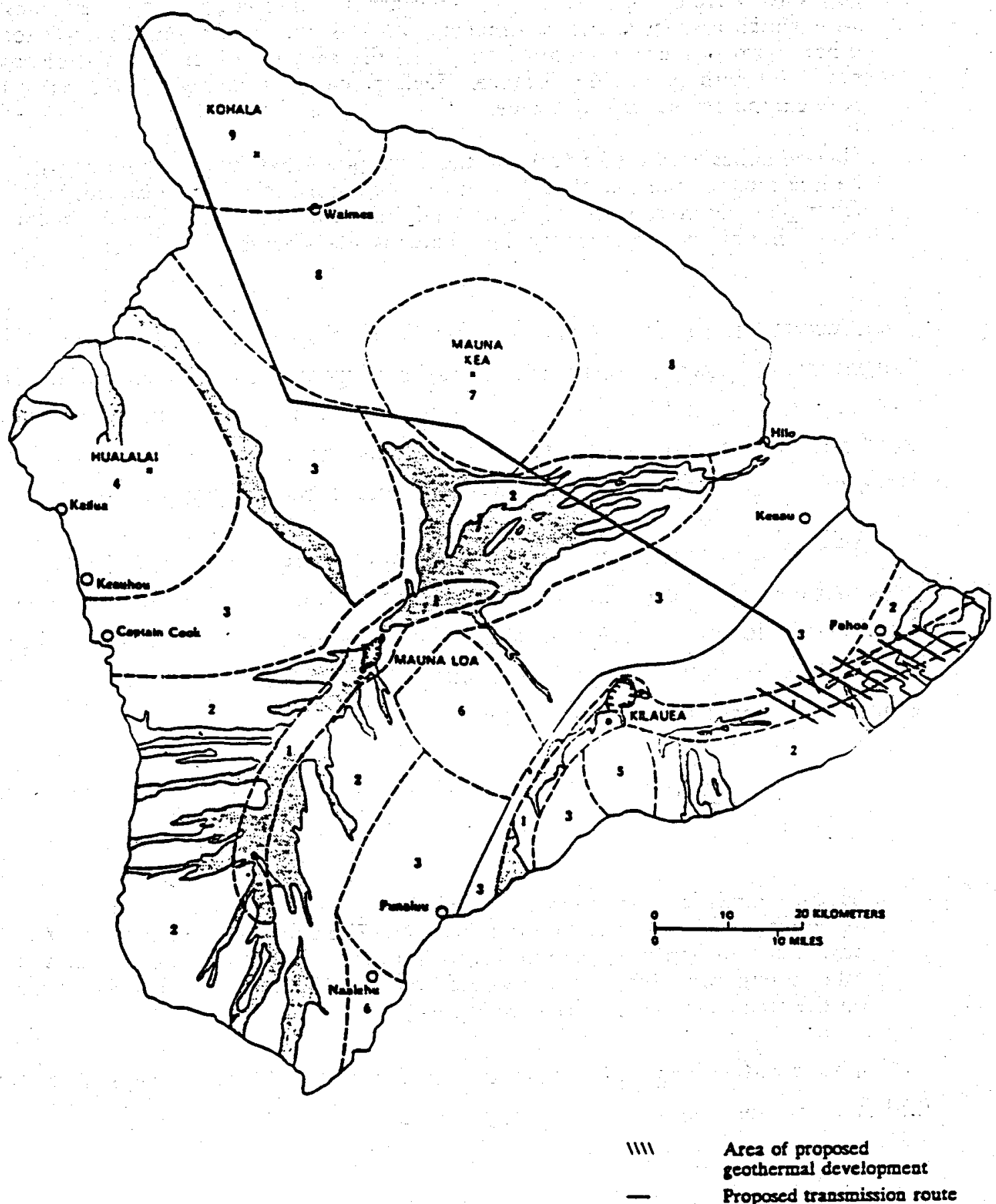

po

Source: Volcanism In Hawaii, U.S. Geological Survey Professional Paper 1350. 
Figure 8 indicates that the geothermal plants will be located in or near Hazard Zone 1, while the transmission lines will traverse Hazard Zone 2 Lava flow hazard zones are described as:

\begin{abstract}
"Zone 1 consists of the summit areas and active parts of the rift zones of Kilauea and Mauna Loa; in those areas, $25 \%$ or more of the land surface has been covered by lava within historical time, during the 19th and 20th centuries. These areas contain the sites of most historical eruptions, and a large majority of the lava flows that will affect other zones on Kilauea and Mauna Loa in the near future probably will originate in Zone 1
\end{abstract}

Zone 2 consists of several areas that are adjacent to and downslope from the active rift zones of Kilauea and Mauna Loa and therefore are subject to burial by lava flows of even small volume erupted in those rift zones. On Kilauea south of its east rift zone. as much as $25 \%$ of the land surface has been covered by lava during historical time. and $0-15 \%$ has been covered since 1950. Lava ilows have covered parts of this area as recently as January 1986, and the history of Kilauea suggests that they will continue until some significant change occurs within the volcano. Although very little of the area in Zone 2 north of the lower east rift zone of Kilauea has been affected by lava since 1950 , about $15 \%$ of that surface has been covered during historical time. On Mauna Loa, long and voluminous lava flows have repeatedly entered the areas included in Zone 2, covering about $5 \%$ of those areas since 1950 and about $20 \%$ within historical time."

In addition to lava flows, ground fractures and subsidence will also place power plant structures at risk, especially in the Kilauea rift zone area. Figure 9 is the hazard zone map for ground fractures and subsidence. The zene of highest hazard, Zone 1 , includes the summit areas and rift zones of Mauna Loa and Kilauea, where fracture and subsidence occur most frequently. On Kilauea, the geothermal plants will be located in or near this zone. Zone 2 consists of the south flank of Kilauea, where fracturing and subsidence occur somewhat less frequently than in the summit and rift zone areas. Again, from the previousiy cited U.S.G.S. report, the danger is apparent:

"Large parts of the flanks of Kilauea and Mauna Loa. for example, sometimes subside abruptly. The areas affected may be several tens of kilometers long and involve hundreds of square kilometers of land."

The extent of the danger these hazards present to the project can be considerable especially over the long term. The U.S.G.S. report estimates that 
Figure 9

\section{HAZARD ZONES FOR GROUND FRACTURES AND SUBSIDENCE ON THE ISLAND OF HAWAII}

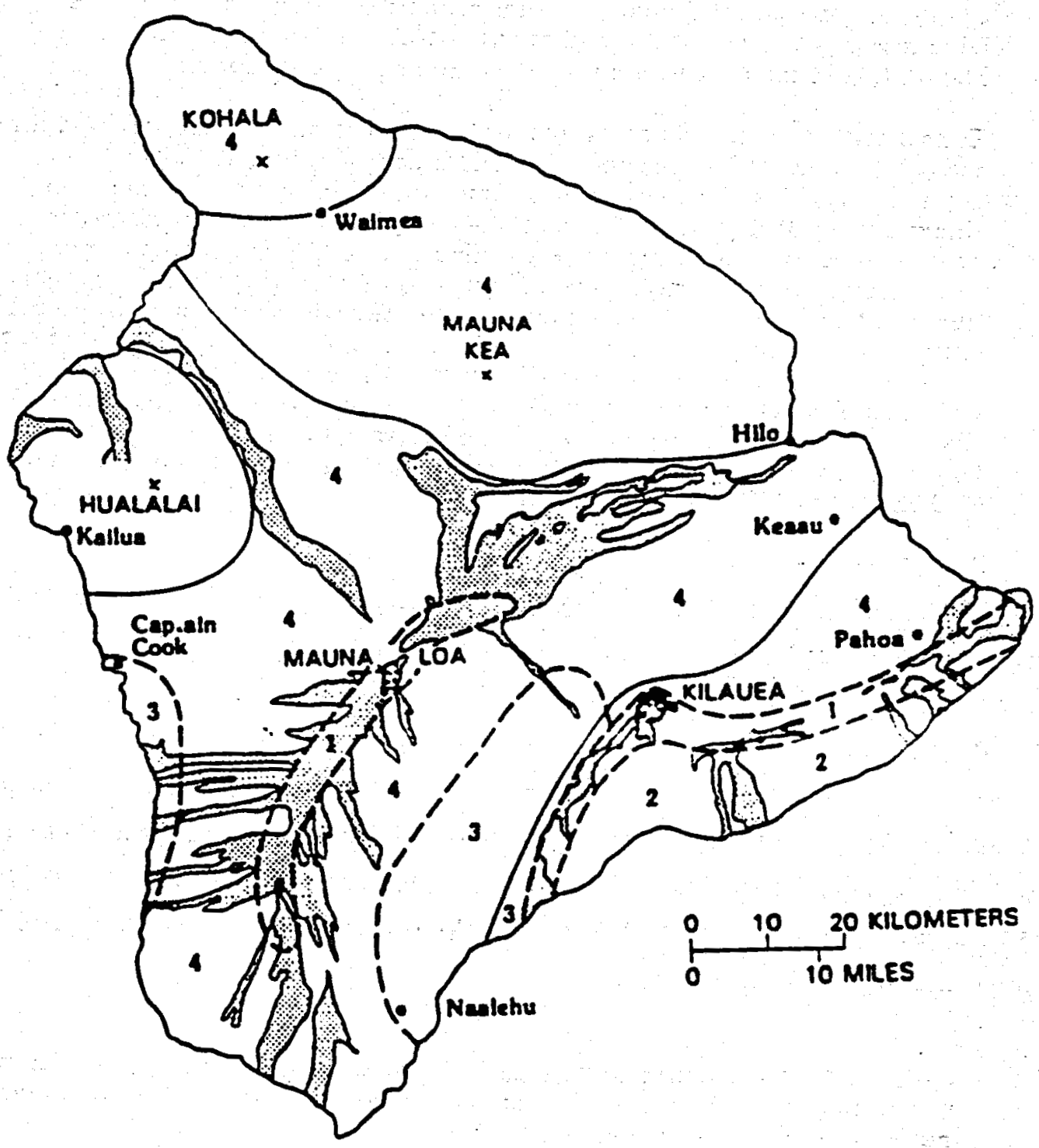

Source: Volcanism In Hawaii U.S. Geological Survey Professional Paper 1350. 
about $5-10 \%$ of Kilauea and Mauna Loa could be covered with lava during any 50 year period. If 10 to 20 geothermal plants are located along Kilauea's rift zone, it does not seem unreasonable to assume that one or more may be affected by either lava flows or ground subsidence over a 40-year period. Mitigation procedures such as lava flow diversions, channels, or barriers may be temporarily effective in some cases but can create legal and social problems if the flows are diverted to an area which otherwise would have been spared. These structures would have to be quite large since lava flows can easily be more than 10 to 20 feet thick and they most likely would have to be earthen structures capable of withstanding the tremendous heat and pressure of large lava flows. Building on high ground can also be effective temporarily, but since lava flows move in unusual ways and change the elevation of the land. what was safe in one year may not be safe the next. The unusual movements of lava flows are described in the previously cited U.S.G.S. report:

The paths followed by lava flows are generally downslope, but they may vary in detail Because parts of a flow are continually cooling and becoming more viscous, the flow may not move directly into the lowest available ground as would a stream of water. Lava flows may move diagonally down slopes or even cross low ridges."

A proposal to design parts of the plant for mobility and to move them about when danger is imminent will require specialized designs. moving equipment and assumes enough warning time to shut the plant down, disassemble it, and move the components to a safe place. The result is still the loss of a plant site and generating capacity. The State report assumes that destroyed plants will be rebuilt immediately, but this will depend on the magnitude and length of the eruption and assumes a replacement site and wellfield is readily available. If this assumption is wrong any components saved from destruction could not be used until new wells had been drilled and a safe wellfield and plant site located and developed.

Whatever shape the mitigation structures take their cost will be

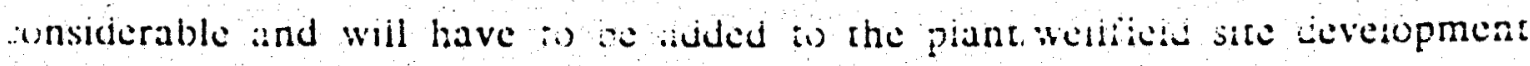
costs. None of these special design features are considered in the State report or are adequately considered in this updated NEA report. 
The best advice for building in these hazardous areas comes from the U.S.G.S. report:

\begin{abstract}
"Protection from the effects of lava flows, other than by such methods of diversion or control, is generally not feasible. An individual lava flow will have roughly the same effects all the way from its source to its terminus, and attempts to protect buildings and other structures from the hot, crushing lava generally are not effective."

"Avoidance through land-use zoning and evacuation is virtually the only way to reduce losses from lava flows."
\end{abstract}

The inability to control these hazards has led the U.S.G.S. to recommend that facilities that have unusual value or are essential to public health and safety should not be built in areas where hazards are high. Power piants are nor oniy essential to the public health and safety of a community, but they provide the lifeblood required for the maintenance of a healthy, productive society. It seems unwise to locate them in areas where their functioning is likely, at best, to be disrupted or, at worst, to be completely destroyed. 


\section{NEA - PROJECT INTEREST RATES}

The cost comparison and present value analysis is done in constant dollars using "real" rates of interest. The objective of expressing costs in constant dollars is to remove the effects of inflation over time and to allow a comparison of costs using a common point as a reference. The term "real" when referring to interest rates means the rate is net of (without) inflation.

The analysis assumes the entity proposing to develop the geothermal resource will sell bonds to pay for it. To be consistent with the DAHI report, 24 year corporate utility bonds are used in the analysis although according to some experts 24 years is too long a period for a venture such as this. ${ }^{1}$

Four different interest rates are used in the analysis, 1) a low risk bond rate for the construction of new oil or solar/oil generating facilities, 2) a high risk bond rate for the construction of geothermal generating facilities, 3) a long-term U.S. Treasury bond rate to provide a next best alternative investment and to act as a basis for comparing the present net values of the three power generation alternatives, and 4) a short-term U.S. Government security rate for the highly liquid plant replacement insurance fund.

The interest rates used in the analysis can be found in Table 12 . The 10 year average real rate is used in the analysis Aaa Public Utility bond rate was selected for oil and solar/oil alternatives. This rate assumes the risk for the investor is low and therefore the cost to the entity issuing the bond is also low. Bond insurance increases the rate by 0.0025 .

The Baa bond rate is used for geothermal investment. The Baa bond is a higher risk bond. Since the geothermal venture is higher in risk than the other alternatives a higher return will have to be guaranteed to investors for their participation. A higher raturn to the inyestor means a highet cost $\therefore$ the issuing entity. Bond insurance is high risk as well and increases the rate by 0.01 .

1V Alex Sifford, Geothermal Program Manager, Oregon Department of Energy, in his review of the DAHI report, June, 1988 
Table 12

DNTEREST RATES 1

\begin{tabular}{|c|c|c|c|c|c|c|c|c|c|c|}
\hline Year. & \multicolumn{2}{|c|}{$\begin{array}{c}\text { Public } \\
\text { Utility Bonds }\end{array}$} & \multicolumn{2}{|c|}{$\begin{array}{l}\text { Public } \\
\text { Utility Bonds }\end{array}$} & $\begin{array}{l}\text { Bond } \\
\text { Risk } \\
\text { Factor }\end{array}$ & \multicolumn{2}{|c|}{$\begin{array}{c}\text { US } \\
\text { Treasury Bonds } \\
\text { Earaing Rate }\end{array}$} & \multicolumn{2}{|c|}{$\begin{array}{c}\text { US } \\
\text { Govamt Securities } \\
\text { Earaing Rate }\end{array}$} & $\begin{array}{l}\text { Aanual } \\
\text { Inflation } \\
\text { Rate }\end{array}$ \\
\hline & Nominal & Real & Nominal & Real & & Nominal & Real & Nominal & Real & Real \\
\hline 1974 & $8 \pi$ & 281 & 984 & 394 & 213 & 6.98 & 108 & 7.94 & 204 & 5.90 \\
\hline 1975 & 9.03 & -0.27 & 10.96 & 166 & 193 & 6.98 & -232 & 6.09 & -321 & 930 \\
\hline 1976 & 8.63 & 223 & 982 & 3.42 & 219 & 6.78 & 0.38 & 525 & -2.15 & 6.40 \\
\hline 1977 & 8.19 & 149 & 9.06 & 236 & 0.87 & 7.06 & 0.36 & $5 s 3$ & -217 & 6.70 \\
\hline 1978 & 8.87 & 157 & 9.62 & 232 & 0.75 & 789 & 0.59 & 758 & 0.28 & 730 \\
\hline 1979 & 9.86 & 0.96 & 10.96 & 206 & 110 & 8.74 & -0.16 & 10.06 & $L 16$ & 8.90 \\
\hline 1980 & 1230 & 330 & 13.95 & 4.95 & 1.65 & 10.81 & 181 & 11.37 & 237 & 9.00 \\
\hline 1981 & 14.64 & 4.94 & $\$ 6.60$ & 5.90 & 296 & 1287 & 3.17 & 380 & $\therefore .20$ & $9: 0$ \\
\hline 1982 & 14.22 & 782 & 16.45 & 10.05 & 223 & 1223 & 5.83 & 1107 & 4.67 & 6.40 \\
\hline 1983 & 1252 & 8.62 & 1420 & 1030 & 168 & 10.84 & 6.94 & 8.73 & 483 & 3.90 \\
\hline 1984 & 1272 & 9.02 & 1453 & 1083 & 181 & 11.99 & 8.29 & 9.76 & 6.06 & 370 \\
\hline 1985 & 11.68 & 8.68 & 1296 & 996 & 128 & 10.75 & 7.75 & 765 & 4.65 & 300 \\
\hline 1986 & 8.92 & 6.22 & 10.00 & 730 & 108 & 8.14 & 5.44 & 6.03 & 333 & 270 \\
\hline 1987 & 9.52 & 6.22 & 10.53 & 723 & 101 & 8.63 & 533 & 603 & 273 & 330 \\
\hline 1988 & 10.05 & 6.95 & 1200 & 790 & 0.95 & 898 & 588 & 609 & 299 & 3.10 \\
\hline S yr. avg. & 1058 & 7.42 & 1180 & 8.64 & 123 & 9.70 & 654 & 7.11 & 3.95 & 3.16 \\
\hline 10 gr. ave. & 1164 & 6.27 & 13.12 & 7.75 & 1.47 & 10.40 & 503. & 9.06 & 369 & 5.37 \\
\hline 15 yr. avg. & 10.66 & 4.70 & 1203 & 608 & 137 & 931 & 336 & 820 & 225 & 595 \\
\hline
\end{tabular}

y Moody's Public Utility Manual." Moodv's Investors Service. 1989, and US. Statistical Abstraet.

The U.S. Treasury bond rate is used as a discount rate to compare the present values of the total $\mathbf{4 0}$ year costs of the three alternatives.

The short-term U.S. Government security rate (six month treasury bills) is used as the rate at which the plant replacement fund earns interest since the funds must be liquid and accessed quickly if needed. 


\section{NEA - PROJECT INSURANCE RATES}

Determining the cost of insuring the geothermal project is difficult since the hazards present a unique situation, and while anything is insurable the question is at what cost? The two most likely causes of damage to the power plants and well fields are lava flows and ground subsidence, while sea-floor earthquakes would be the most likely cause of damage to the undersea cable. (See NEA - Geothermal Project Hazard Assessment section.)

To get an idea of what it would cost to insure the power plants and their faciliries, commercial insurance representatives were contacted at the Fred $S$. James Company, an insurance bookerage firm in Seattle and Los Angeles. They said that perhaps Lloyds of London or AIG would be the type of company that could handle a venture as large as the geothermal project but, without much more information on the specifics of the project (i.e. hazard frequency and intensity, plant location, plant construction details, specific in-place mitigation procedures), no accurate estimate of project insurance cost could be made or even attempted. They did indicate however, that the cost of the insurance would be quite high if it was available at all. None of the representatives was aware of anyone currently offering insurance against the specific types of hazards described (lava flows, ground subsidence, etc.).

There is, however, some parallel between lava flow hazard and floodplain hazard. In both cases damage is caused by a moving liquid material, but while flood damage need not be total, lava flow damage most assuredly is.

To explore this option the Federal Emergency Management Agency (FEMA) in San Francisco was contacted. ${ }^{2}$ While FEMA does not insure against lava flows (and is not aware of anyone who does), it does issue flood plain insurance up to a value of $\$ 200,000$ per structure. The rates vary depending on

1) Phone conversation with Craig Brandt, Fred S. James Company, Seattle, WA January - February, 1989.

21 Phone conversation with John Eldridge, Federal Emergency Management Agency, San Francisco, California, February, 1989. 
flood frequency, intensity and the location of the structure in relation to the maximum height of the flood waters. Some rates for worst case flood scenarios are shows in Table 13).

Table 13

FLOOD INSURANCE RATES 100 YR FLOOD

\begin{tabular}{lll}
\hline & $\begin{array}{l}\text { Annual } \\
\text { Rate } \\
\$ 100 \text { Value }\end{array}$ & $\begin{array}{l}\text { Location of Floor Level } \\
\text { Below Maximum Height } \\
\text { Of Floodwater }\end{array}$ \\
\hline \hline$\$ 265$ & 5 feet \\
& $\$ 385$ & 6 feet \\
& $\$$ feet \\
\hline \hline
\end{tabular}

The highest rate quoted was $\$ 6.60$ per $\$ 100$ value for structures at the lowest level below maximum floodwater, while the lowest rate was $12 t$ per $\$ 100$ value if the lowest level of the structure was built three feet above the maximum height of the floodwater.

Since lava flows are much more destructive than floodwaters and can easily reach thicknesses of ten feet or more, mitigation against them is costly and at best uncertain (see Geothermal Project Hazard Assessment section), it is likely that insurance costs to protect against them would be at least at the $\$ 6.60$ total destruction level for a 100 year frequency event.

If this rate ( $\$ 660$ per $\$ 100$ value) is applied to the entire value of a $25 \mathrm{MW}$ power plant whose replacement cost is between $\$ 100$ million and $\$ 130$ million (our low and high plant/well field development costs as generated in the CENTPLANT model) the annual insurance cost would be between $\$ 7.1$ and $\$ 8.6$ million per $25 \mathrm{MW}$ plant or between $\$ 142$ million and $\$ 172$ million dollars for the entire project (twenty $25 \mathrm{MW}$ plant/wellfield sites) at full development. At this rate the cost of insurance over the life of the project easily exceeds the cost of replacing all the plants and facilities. Clearly, this would not be the preferred option.

1. Volcanism In Hawaii, Chapter 22, U.S. Geological Survey Professional Paper 1350, 1986. 
The only other option open is self-insurance, or absorbing the losses internally. If the self-insurance option were taken the project owners would have to not only make good on losses to bond holders but would have to pay for the immediate replacement of lost generating capacity. It may prove quite difficult to sell bonds to finance a new geothermal plant to be built in an area containing hazards identical to one that had just been destroyed by lava flows or ground subsidence. Bond buyers and insurers would probably be a bit more skeptical of the safety of their investment the second time around and would probably require an even higher interest rate to cover their risk than the initial of fering which was a higher risk bond to begin with.

If it proved difficult to issue bonds at a reasonable rate, money would have to be available from some other source in an amount large enough to insure that the reserve capacity of the system did not remain below critical levels for a long period of time. Output from existing geothermal plants could not simply be increased to replace the lost power since the geothermal plants would already be operating at their maximum output capacity. The replacement power would have to come from somewhere else, and it would have to come quickly because maintaining adequate reserve generating capacity is critical to public safety.

If, for example, total generating capacity were $2000 \mathrm{MW}$ and $30 \%$ (600 MW) were required to be held in reserve, $70 \%(1400 \mathrm{MW})$ would be available for the system's annual load. The reserve is there to handle peak loas and emergenc: demands that may occur. If some geothermal capacity were lost, reserve capacity would drop as well as shown in Table 14 .

If $50 \mathrm{MW}$ is lost the reserve margin drops to $28 \%$; if $100 \mathrm{MW}$ is lost it drops to $26 \%$. If we are looking at $25 \mathrm{MW}$ plants and $2-4$ plants are lost, the amount of capital needed immediately to bring the reserve capacity back up to the $30 \%$ level would be half a billion dollars. If 6 plants were lost, about three quarters of a billion dollars would be needed in a short period of time. 
Table 14

\begin{tabular}{|c|c|c|c|c|}
\hline \multicolumn{5}{|c|}{$\begin{array}{l}\text { GENERATING CAPACITY AND RESERVE } \\
\text { Capacity Relationships }\end{array}$} \\
\hline Total Capacity (MW) & 2000 & 1950 & 1900 & 1850 \\
\hline Peak (MW) & 1400 & 1400 & 1400 & 1400 \\
\hline Reserve Capacity (MW) & 600 & 550 & 500 & 450 \\
\hline Reserve Margin & $30 \%$ & $28 \%$ & $26 \%$ & $24 \%$ \\
\hline MW Loss & 0 & 50 & 100 & 150 \\
\hline MW/Plant & 25 & 25 & 25 & 25 \\
\hline \# Plants & 0 & 2 & 4 & 6 \\
\hline MS/Plant Low & & 107.79 & 107.79 & 107.79 \\
\hline MS/Plant High & & 129.40 & 129.40 & 129.40 \\
\hline MS Total Capital Low & & 21558 & 43116 & 646.74 \\
\hline MS Total Capital High & & 25880 & 517.60 & 776.40 \\
\hline
\end{tabular}

Either insurance, self-insurance or some combination of the two will require immense sums of money and presents a formidable obstacle in terms of costs to be shouldered by ratepayers. In the analysis a worst case scenario is assumed and bonds cannot be sold to finance replacement power plants. A replacement plant fund is established beginning in year three to insure that money is available to replace one-half the power plants and one-third the cable system exposed to geologic hazards. The fund is increased accordingly as plants come on line until full development is reached in the twenty-first year. The money is then expended to replace power plants and transmission facilities lost in years $22,30,34$ and 35 . In the $25 \mathrm{MW}$ scenario eight plants are lost, two in each year, while in the $50 \mathrm{MW}$ scenario four plants are lost. Since the bonds initially issued are insured, remaining bond payments on each plant are eliminated as the plants are lost. In addition, the fund earns interest but since it must be reasonably liquid and cannot be tied up in long-term investments it earns interest at a rate equal to short-term U.S. Government securities (six month Treasury bills).

This risk level is based on the high level a encertainty in predicting volcanic activity in the development area as described in the U.S.G.S. report cited in the earlier chapter on geologic hazards. The report describes the prediction of volcanic activity and lava coverage as follows: 
"Based on historical records, about 5-10 percent of Kilauea and Mauna Loa could be covered during any 50 -year period. Although wide fluctuations can be expected in eruptive rates from one decade to another, the overall rates likely will remain about the same. It is not possiole. however. to predict where the next eruptive centers will be, how frequent or copious eruptions will be in a specific area, or which specific areas will be covered by lava.

The volcanic activity along Kilauea's east rift zone in historical time illustrates a difficulty in using the short historical record to predict future activity in a specific area. Between 1800 and 1950, approximately 2 percent of the eastern flanks of the volcano had been covered by lava from the east rift zone. In 1950, the probability based on these figures that a site in that region would be covered would have been 0.013 percent per year. However, between 1950 and 1975 about 8 percent of Kilauea's east flank was covered by lava, and so the coverage in that interval was actually about 0.32 percent per year.

Estimates of future coverage may be no more accurate."

In the description cited above actual lava coverage was 25 times as extensive as would have been predicted based on historical records. Given the axiom that risk becomes greater as uncertainty becomes greater, that low probability events can and do occur, and given the nature of the hazards associated with the area of the proposed development, our assumption of a one-half plant replacement fund at the worst case scenario and a $40 \%$ plant loss does not seem unreasonable- 



\section{NEA - THE PRICE OF RESIDUAL FUEL OIL}

Determining future world oil prices is difficult because it depends greatly on the level of world oil production, future oil exploration and discovery, and market responses (demand, substitution etc.) to oil prices over the long term.

It is generally agreed, and time has shown, that the price of oil is crucial to the availability and price of all other energy. A Wall Street Journal article ${ }^{1}$ discussing petroleum use states that while there is still concern about OPEC domination of oil resources in the future, other forces will act to keep prices in check. The article puts it this way:

Some suggest this could set the stage for a return of the political upheavals and price escalation of the 1970s. But others say leading members of OPEC such as Saudi Arabia are convinced that relatively low oil prices are in their best interests in the long term. They don't want to spur oil exploration in * other areas-in the high-cost U.S, for example, where production has rapidly declined since the 1986 crash-or lose customers to alternate energy sources likely to be launched on the next petroleum price spike.

But relatively low oil prices will discourage investment in research and development of alternate energy sources. That's because many potential alternatives, which might be competitive with $\$ 40$ to $\$ 50$ a barrel oil, can't compete on a cost basis with $\$ 18$ a barrel oil.

"In the short run, cheap oil keeps down natural gas and coal," says Robert $H$. Horton, a managing director of British Petroleum Co. "In the longer run, these and others-including our exotic old friends like solar, shale, windmills and the atom-curb excess greed on the part of oil."

The Gas Research Institute ${ }^{2}$ agrees on the effects high oil prices have on other energy sources:

1) The Wall Street Journal Industry Focus, Petroleum Use To Maintain Its Stature, March 1, 1989.

21 The Gas Research Institute, 89 Policy Implications Of The GRI Baseline Projection Of US. Energy Supply And Demand To 2010. 
"GRIs oil price projections have been changed over time in response to new information as the historical years have been added. In retrospect, two major factors have continually been underestimated:

- On the supply side, the response of new non-OPEC supplies to high prices in the 1970 s and the persistence of that supposedly high-cost production in the face of the more recent low price periods; and

- On the demand side, the dramatic reduction in the use of residual fuel oil in stationary energy applications, which was aided in the 1980 s by a period of low energy demand growth (conservation) coupled with over investment in new nuclear and coal-fired electric power generating capacity."

While oil prices may increase from time to time, it is not likely they will increase beyond the point at which other energy sources become available and competitive. Based on the experience of the 1970 s, that point seems to be in the range of $\$ 40$ to $\$ 50$ per barrel.

The same Wall Street Journal article mentioned above goes on to state:

Petroleum prices today average between $\$ 14$ and $\$ 18$ a barrel, half the level of the early 1980 s. The future direction is subject to debate. not surprisingly since a former consensus forecast of $\$ 80$ oil by now was off the mark. Generally, prices are expected to remain somewhat ilat for a tew years and then rise gradually.

Many agree with the Petroleum Industry Research Foundation's energy economists who see prices averaging between $\$ 75$ and $\$ 20$ a barrel, in real terms, through the 1990 s. "We don't see a $\$ 25$ or $\$ 40$ world nor a $\$ 10$ world, but we could still have violent swings," says Lawrence Goldstein, the foundation's executive vice president.

The type of fuel oil used in the plants in the analysis is. residual fuel oil. This is the heavier oil that remains after the distillate fuel oils and lighter hudrocarbons are distilled away in refinery generation. Since the price of residual fuel oil and crude oil are very close on the world market (Figure 10), the report assumes they are equivalent. 
Figure 10

\section{CRUDE OIL AND RESIDUAL. OIL PRICES}

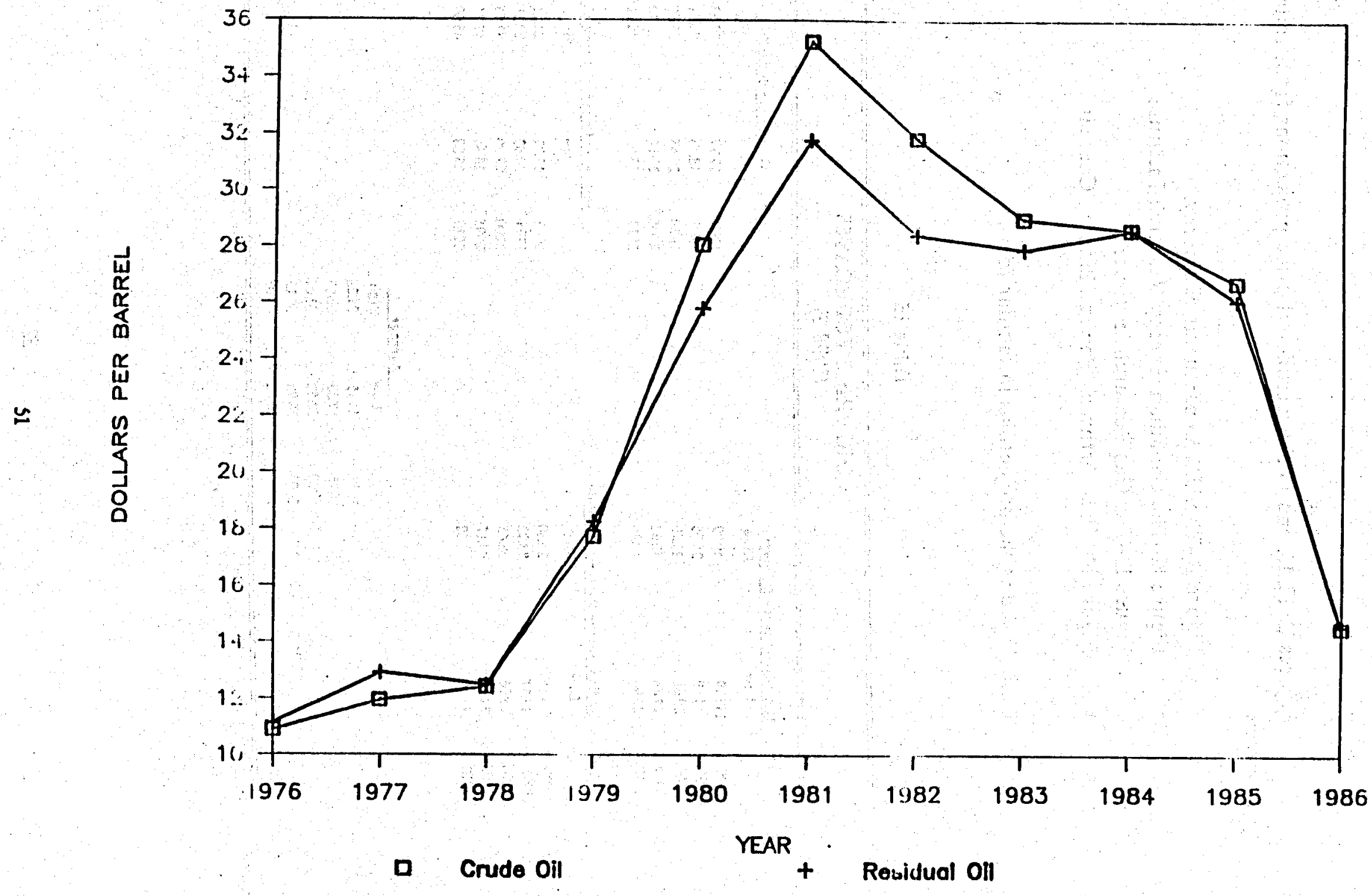


Figures $11,12,13$, and 14 show crude oil price projections from four different sources:

- Figure 11-The U.S. Department of Energy.

- Figure 12-The Canadian Energy Research Institute

- Figure 13-The Gas Research Institute

- Figure 14-The Northwest Power Planning Council

Table 15 shows the projections in tabular form.

Table 15

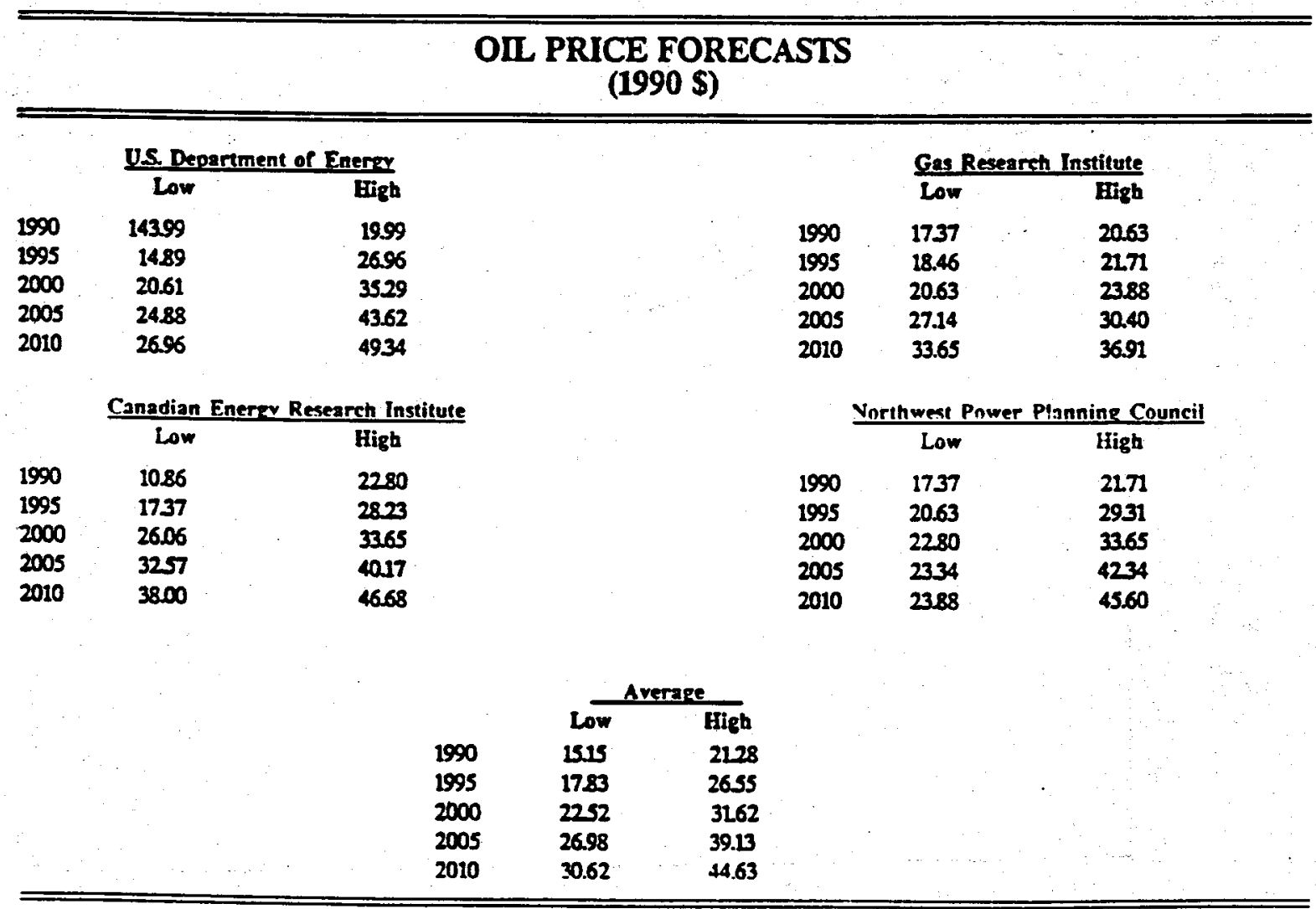


Figure 11

\section{OIL PRICE FORECAST}

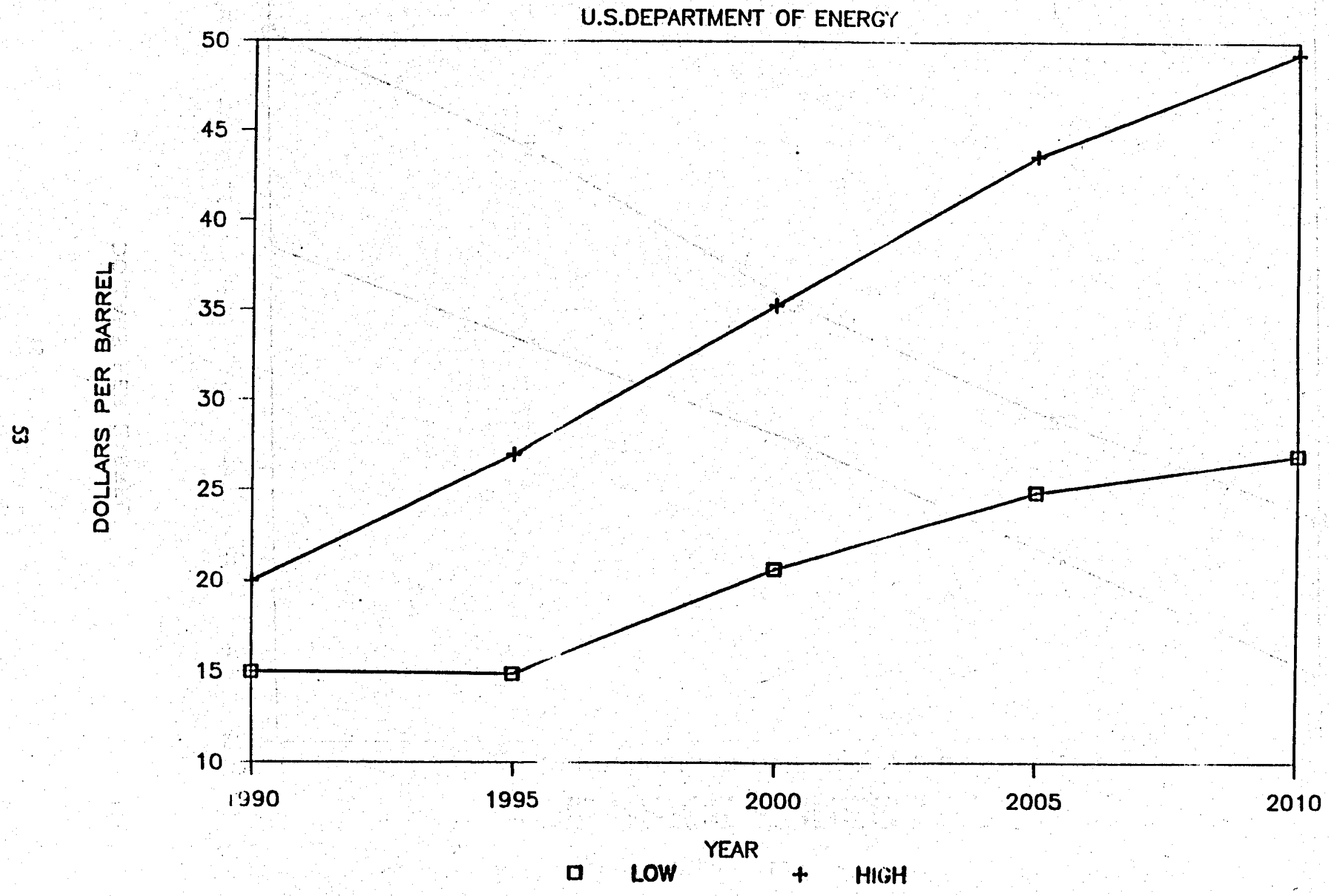


Figure 12

\section{OIL PRICE FORECAST}

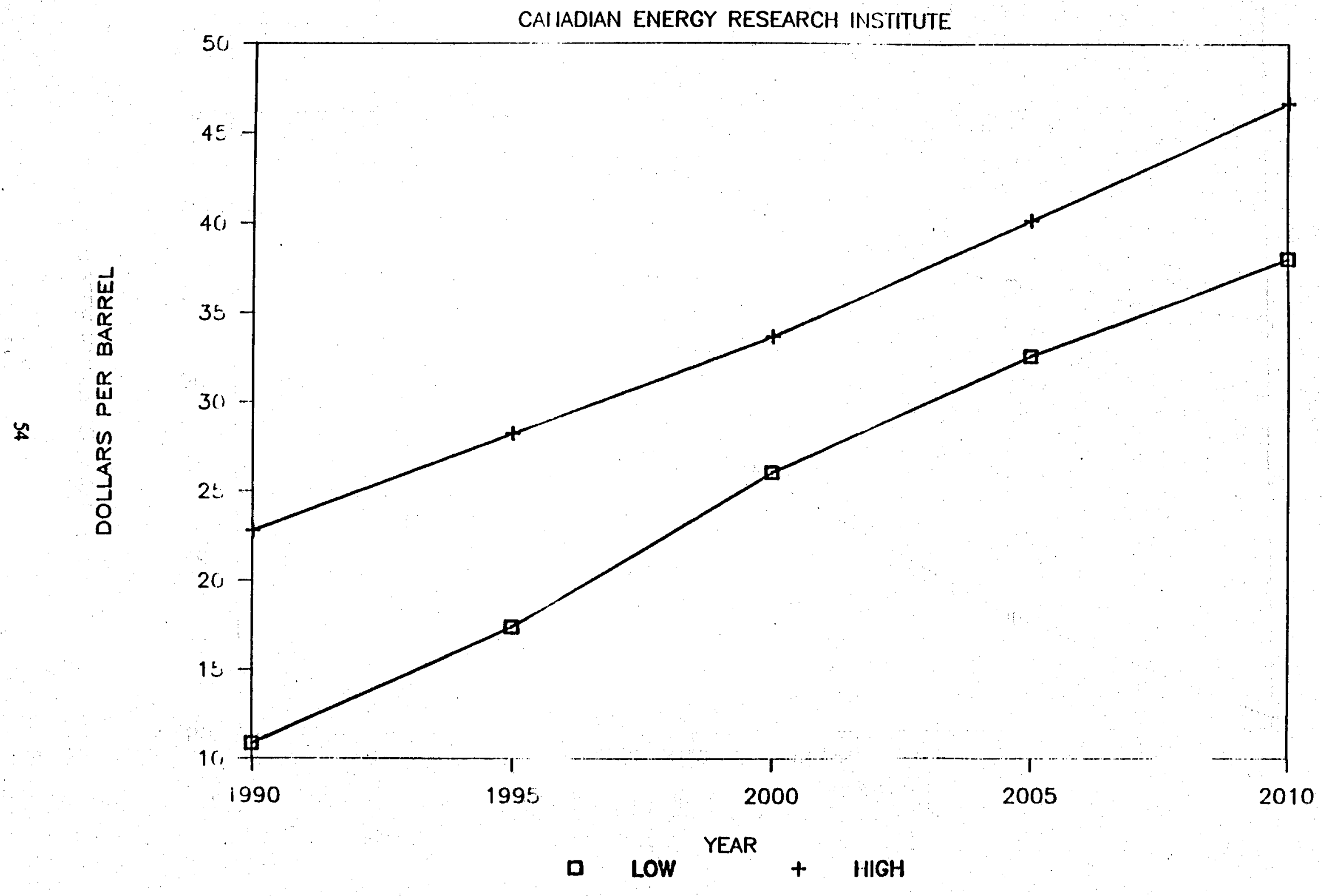


Figure 13

\section{OIL. PRICE FORECAST}

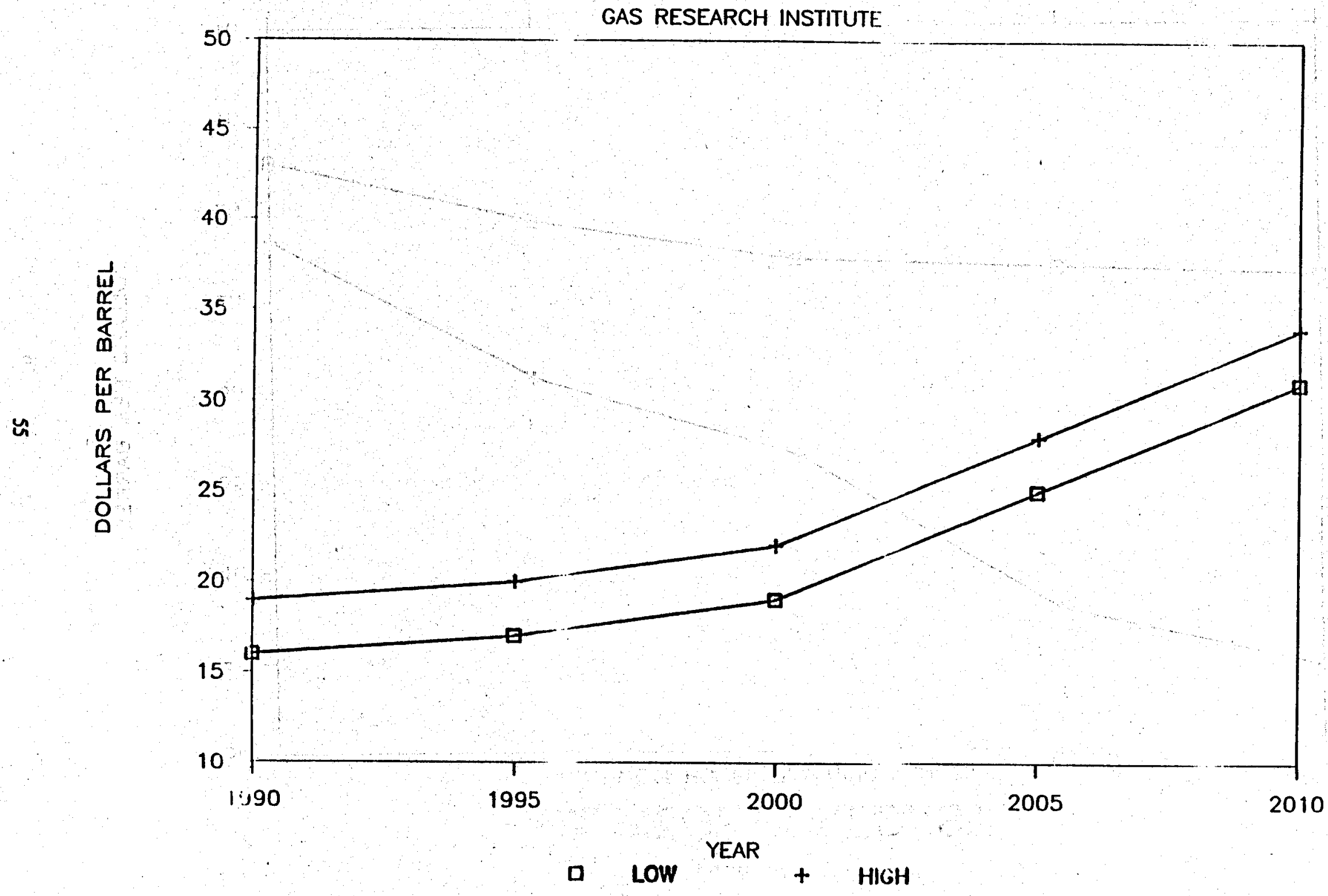


Figure 14

\section{OIL PRICE FORECAST}

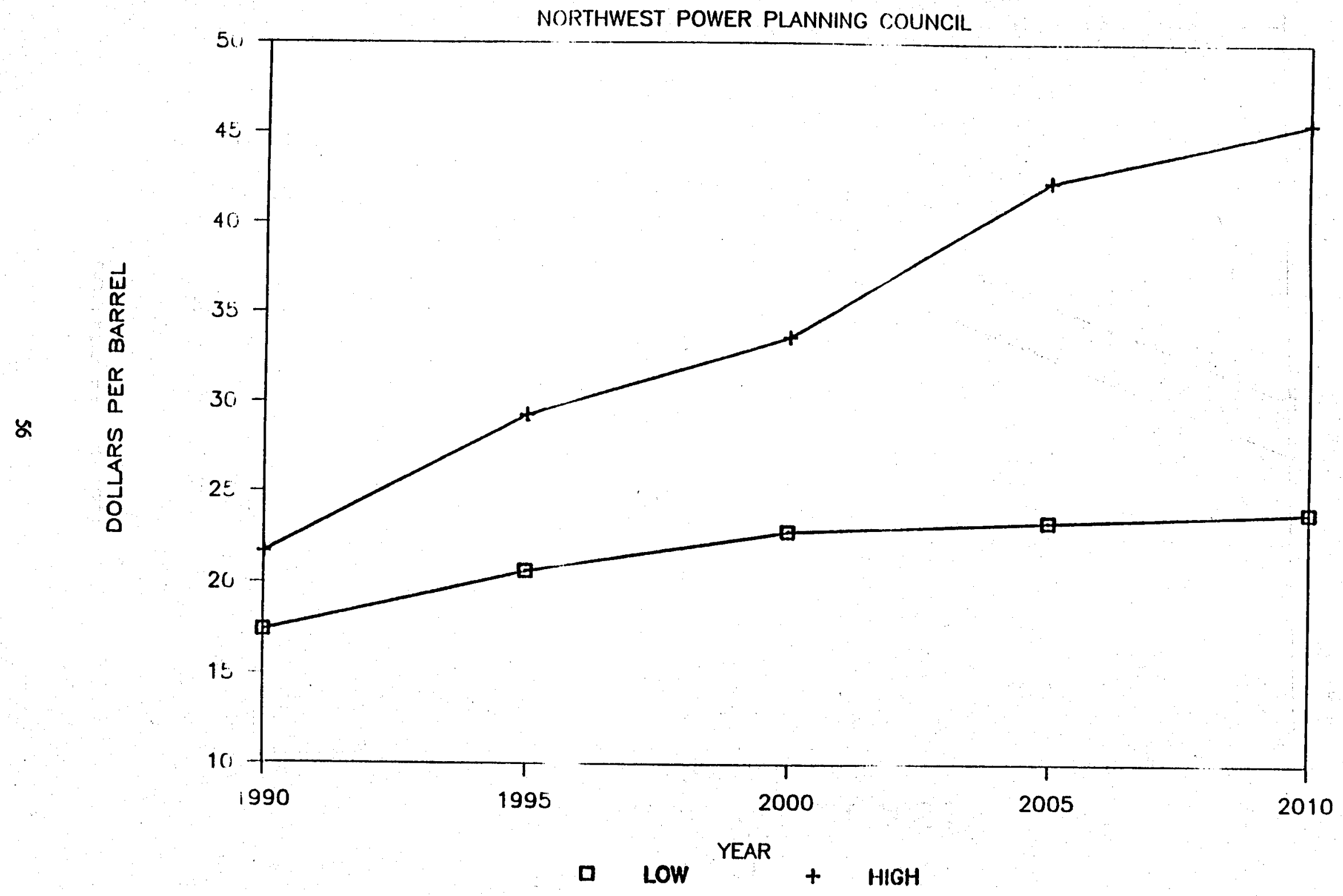


The highest high price projection is from the U.S. Department of Energy at $\$ 49.34$ per barrel in 2010. The lowest low price if from the Northwest Power Planning Council at $\$ 23.88$ per barrel in 2010 . The average of the four sets of projections is $\$ 30.62$ on the low end and $\$ 44.63$ on the high end in 2010.

The oil prices used in the analysis are based on these oil price projections and on the belief that market forces will limit oil prices to below $\$ 50$ per barrel. An oil price of $\$ 25$ per barrel is used as a starting point in the 5th year (1994) and is increased at an annual real rate of $4.0 \%$ until a price of $\$ 45.00$ is reached in the 21st year (2010). The $\$ 45.00$ price is then kept constant throughout the remainder of the analysis period. The oil price is in 1990 dollars. 



\section{NEA - PROJECT COST COMPARISON}

In this analysis the cost of generating 500 net $\mathrm{MW}$ of electricity from the three different sources described earlier is compared:

Generation sources:

Alternative \#1

20 - 25 net MW geothermal power plants

Alternative \#2

$5 \cdot 100$ net MW oil-fired power plants

Alternative \#3

5-100 net MW solar/oil-fired power plants

Alternatives 2 and 3 are also compared to ten 50 net MW geothermal power plants.

The following four scenarios detail the costs of the three generating options used in the analysis. Scenario 1 uses $25 \mathrm{MW}$ power plants at the low plant cost; Scenario 2 uses $25 \mathrm{MW}$ power plants at the high plant cost; Scenario 3 uses $50 \mathrm{MW}$ power plants at the low plant costs, and Scenario 4 uses $50 \mathrm{MW}$ power plants at the high plant costs.

Capital costs used are described in earlier project cost sections. Interest rates, insurance, and oil prices are also described in earlier sections. Other costs used in the analysis are explained below. The project spans a 40 year time period All dollar amounts are in 1990 dollars.

The major line items in the scenarios are explained as follows:

Scenario. describes the scenario being compared.

Year - is the year of the project.

\section{GEOTHERMAL POWER GENERATION}

Geothermal

Development

Timetable (MW)
Is the phasing in of geothermal plants. For $25 \mathrm{MW}$ plants one is brought on line every 12 months. For $50 \mathrm{MW}$ plants one is brought on line every 24 months. $500 \mathrm{MW}$ is on line by the end of the 21st year. 


\section{GEOTHERMAL POWER GENERATION cont'd}

Electricity Produced Is the amount of electricity produced in $1000 \mathrm{MWh}$ assuming the plants operate at $80 \%$ capacity ( $80 \%$ of the time)

Geothermal Plants (MS) Costs of the plants shown in million dollars

Capital

The annual capital costs of the power plants using Baa bond rates. See section NEA - Project Interest Rates.

Replacement Wells

The annual cost to replace or rework production wells.

O\&M

The annual O\&M costs of the power plants. (CENTPLANT Generated)

Royalty

An assessment made by the state on geothermal power production. We assumed an assessment of $10 \%$ on gross power sales after the 8th year of production.

Rent

Rent paid to the state in the amount of $4 \%$ of gross power sales.

Plant Replacement

Fund

Is the fund set aside in lieu of insurance to replace power plants lost to geologic hazards.

Cable and Facilities (MS)

Capital

The annual capital costs of the overland and undersea cable systems using Baa bond rates.

O\&M

The estimated annual O\&M costs of the cable systems. (NEA estimate.)

Administrative

Expenses

Based on administrative costs per $\mathrm{kWh}$ iound in Iawailan Electric Industries, Inc. Annual Report.

Cost

Total annual project costs.

With Profit

Total annual project costs with $8 \%$ profit calculated from Hawaiian Electric Industries, Inc. Annual Report. Profit is calculated as a percent of annual costs.

The second set of costs is in cents per kilowatt hour.

\section{RESIDUAL FUEL OIL POWER GENERATION}

()il Facility

Development

Timetable (MW)

Oil Price

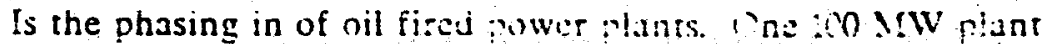
is brought on line every 5 years. $500 \mathrm{MW}$ is on line by the end of the 21st year.

Is the price paid for a barrel of residual fuel oil. See section NEA - The Price of Residual Fuel Oil. 


\section{RESIDUAL FUEL OIL POWER GENERATION cont'd}

Electricity Produced Is the amount of electricity produced in $1000 \mathrm{MWh}$ assuming the plants operate at $80 \%$ capacity ( $80 \%$ of the time).

Oil Consumed

The amount of oil in million barrels it takes to produce the electricity in the line just above. We used a fuel conversion efficiency factor of $35 \%$.

Capital

The annual capital costs of the power plants using Aaa bond rates.

O\&M

The annual O\&M costs of the power plants. (Northwest Power Planning Council estimate.)

Fuel

The annual fuel costs of the power plants (oil consumed times oil price).

Administrative

Expenses

Based on similar costs per $\mathrm{kWh}$ found in Hawaiian Electric Industries. Inc. Annual Report.

Cost

Total annual project cost.

With Profit

Total annual project cost with $8 \%$ profit. Profit is calculated as a percent of annual costs based on Hawaiian Electric Industries, Inc. Annual Report.

The second set of costs is in cents per kilowatt hour.

\section{SOLAR/RESIDUAL FUEL OIL POWER GENERATION}

Solar Facility

Development

Timetable (MW)

Electricity Produced

Solar Produced

Electricity

Oil Price

Oil Produced

Electricity

Oil Consumed
Is the phasing in of oil fired power piants. One $: 00 \mathrm{MW}$ piants is brought on line every 5 years. $500 \mathrm{MW}$ is on line by the end of the 21st year.

Is the amount of electricity produced in $1000 \mathrm{MWh}$ assuming the plants operate at $80 \%$ capacity ( $80 \%$ of the time).

Is the amount of electricity produced in $1000 \mathrm{MWh}$ by the solar generation portion of the facility.

Is the price paid for a barrel of residual fuel oil. See section NEA - The Price of Residual Fuel Oil

Is the amount of electricity produced in $1000 \mathrm{MWh}$ by the oil seneration portion wi the iaciliry.

The amount of oil in million barrels it takes to produce the electricity in the line just above. We used a fuel conversion efficiency factor of $35 \%$.

The annual capital costs of the power plants using Aaa bond rates. 


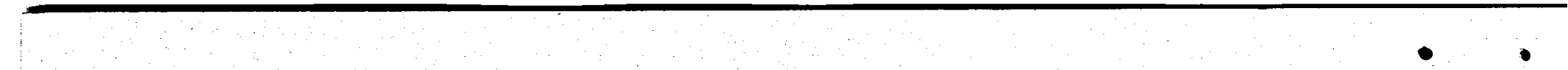




\section{SCENARIO 1}

$25 \mathrm{MW}$ power plants using low plant/wellfield costs and a $20 \%$ contingency 


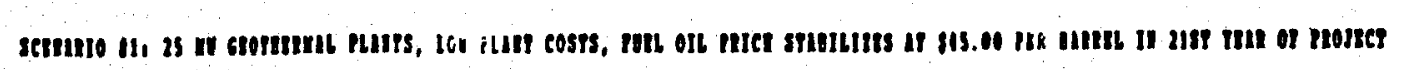

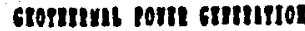

IIII

Genternal berelopent gleetable(m) eteil let Capestty bereloperet

Eletrtelty Proheed (10sim)

cest ios gropment rom nojses

Centlerail tlati (x)

eapleal

Ieplacesent rille

ost

enclty Roguents

Ieat

Hout Ieplocenet Ind

cables on fiefllties (It)

capital

off

Intodetratle stpenes (uSI)

eint (II)

VIit profle (2)

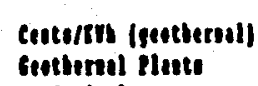

coplent

Ieplueruet vells

oil

toplty pupente

leat Ieplacenest lond

calles une peellities

eipital

ofit

Ihistatutere Bpenes

centertm

centultit (nit) proflt)

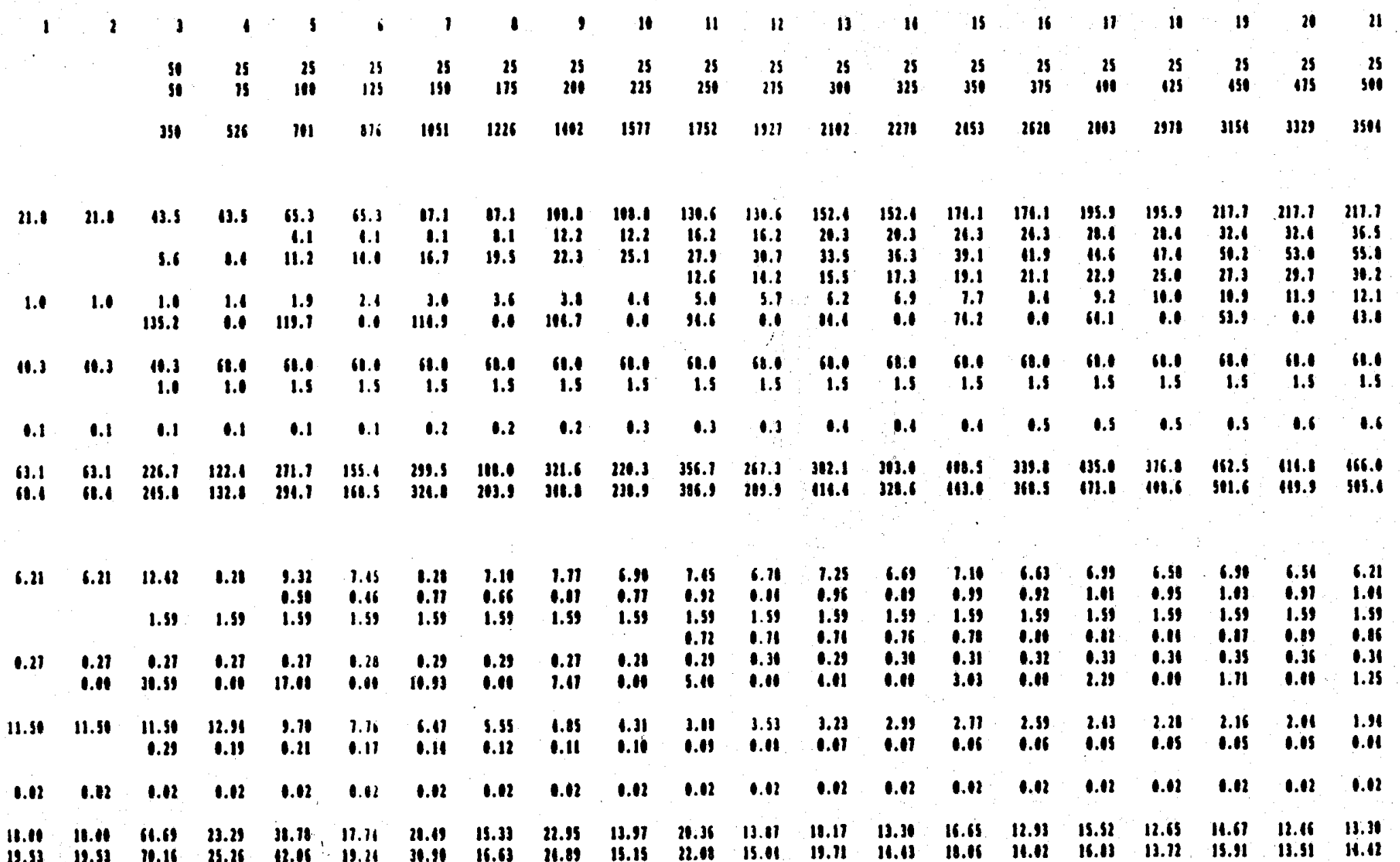


seriniro 11 cons.

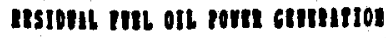

IEI

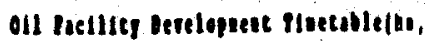
potal set cupelty serelopuent

Oll letee 18 ibll

Ileetrielty irodoced (10mint)

oil conmel (I) bil)

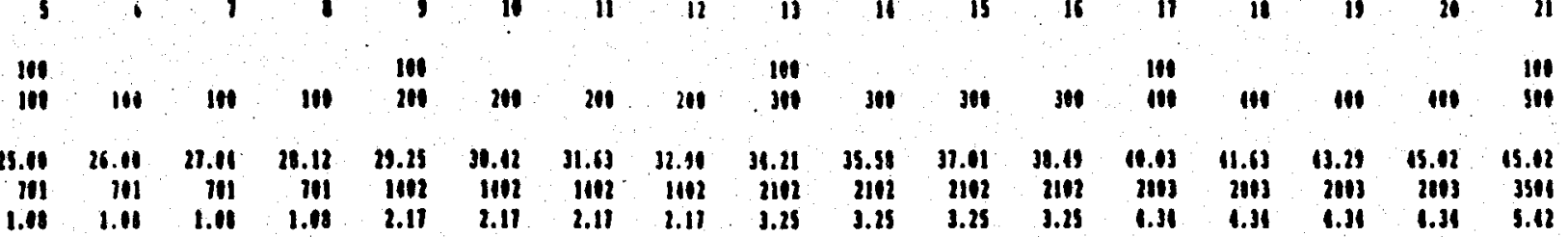

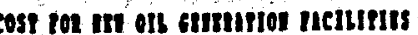

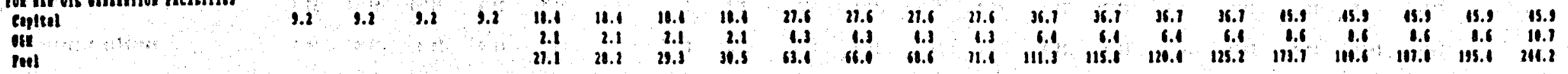

Intolitrative Ingenes (I)!

cont (III)

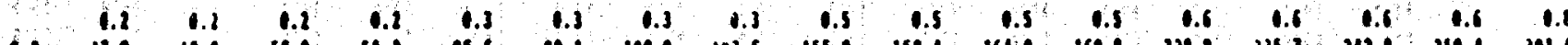

Itt Protic (in)

Cetteirn (bII)

Copteal

Poel

Mentotritie tipenes

rotul centuirn

fotal teterite ulit protit

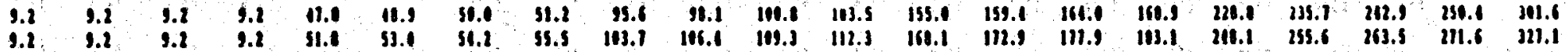

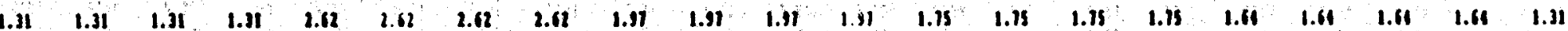

$\begin{array}{llllllllllllllllll}0.31 & 1.31 & 0.31 & 0.31 & 0.31 & 0.31 & 1.31 & 0.31 & 0.31 & 0.31 & 0.31 & 0.31 & 0.31 & 0.31 & 0.31 & 0.31 & 0.31\end{array}$

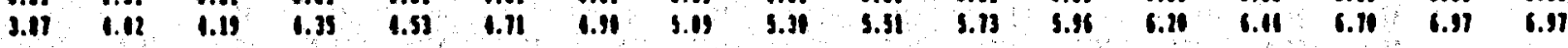

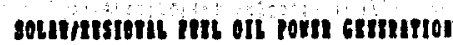
mix

solerroil berclopuent pintebletur Potal int capetif Dertiopieat

Neetrleity Profoed linim

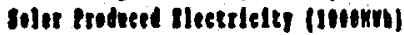

oll Prlee If bil]

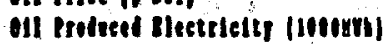

oll counate (I bII)

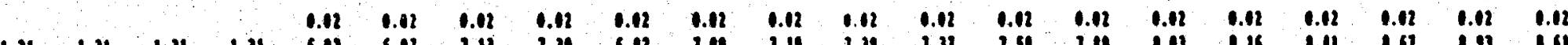

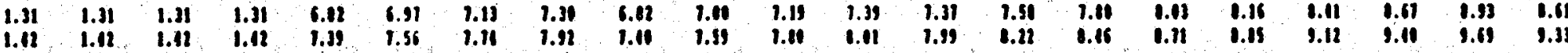

cosf ros soblyost grmunos necturs Capltal

ori

Gialatrative Inpetees (m)

Ihioletreti
Cost (II)

vili Protit (घ)

contenm

int

Indolotratine Inenes

Potil eesteinn

Potal cesturn vith protts

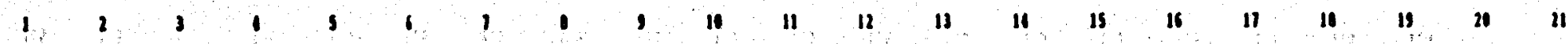

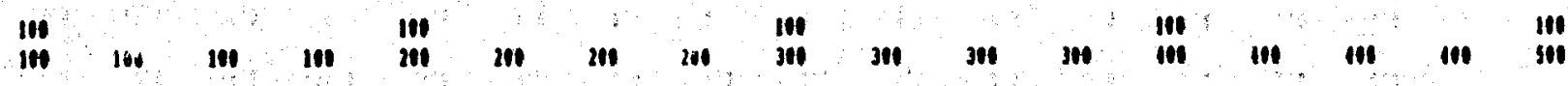

3

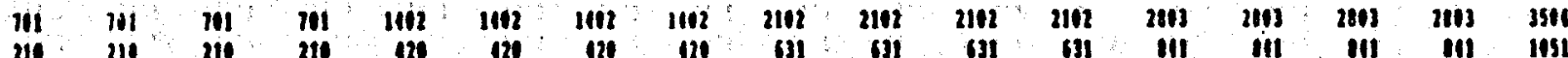

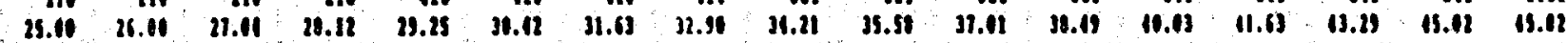

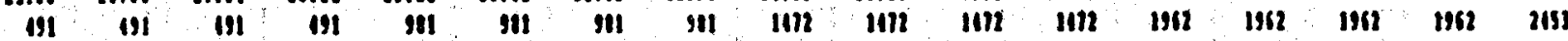

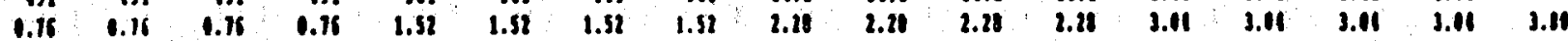

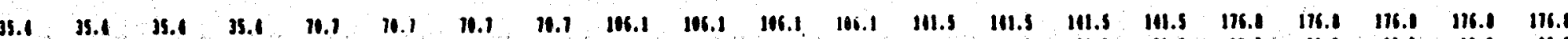

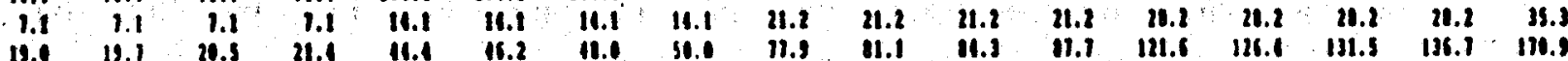

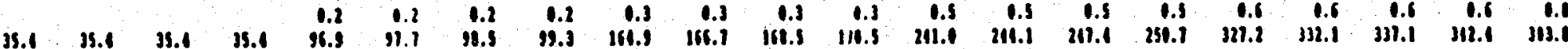

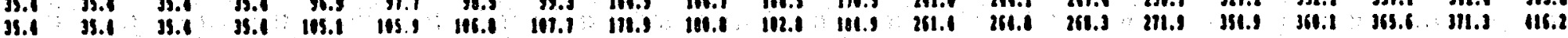

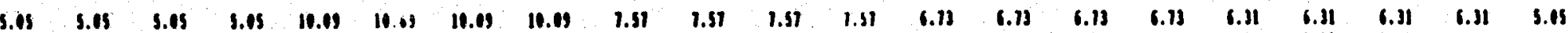

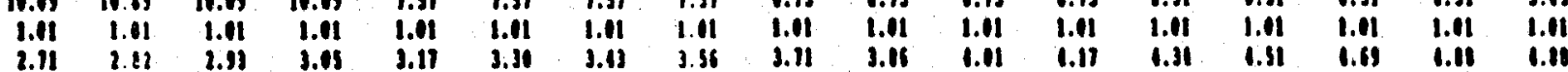

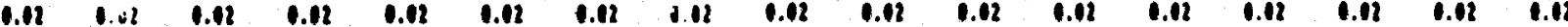

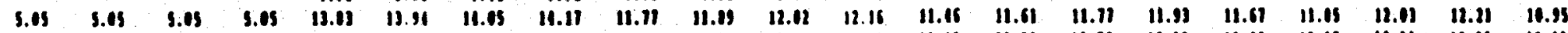

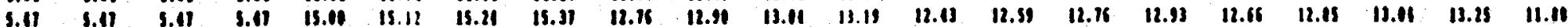


seminio II cort.

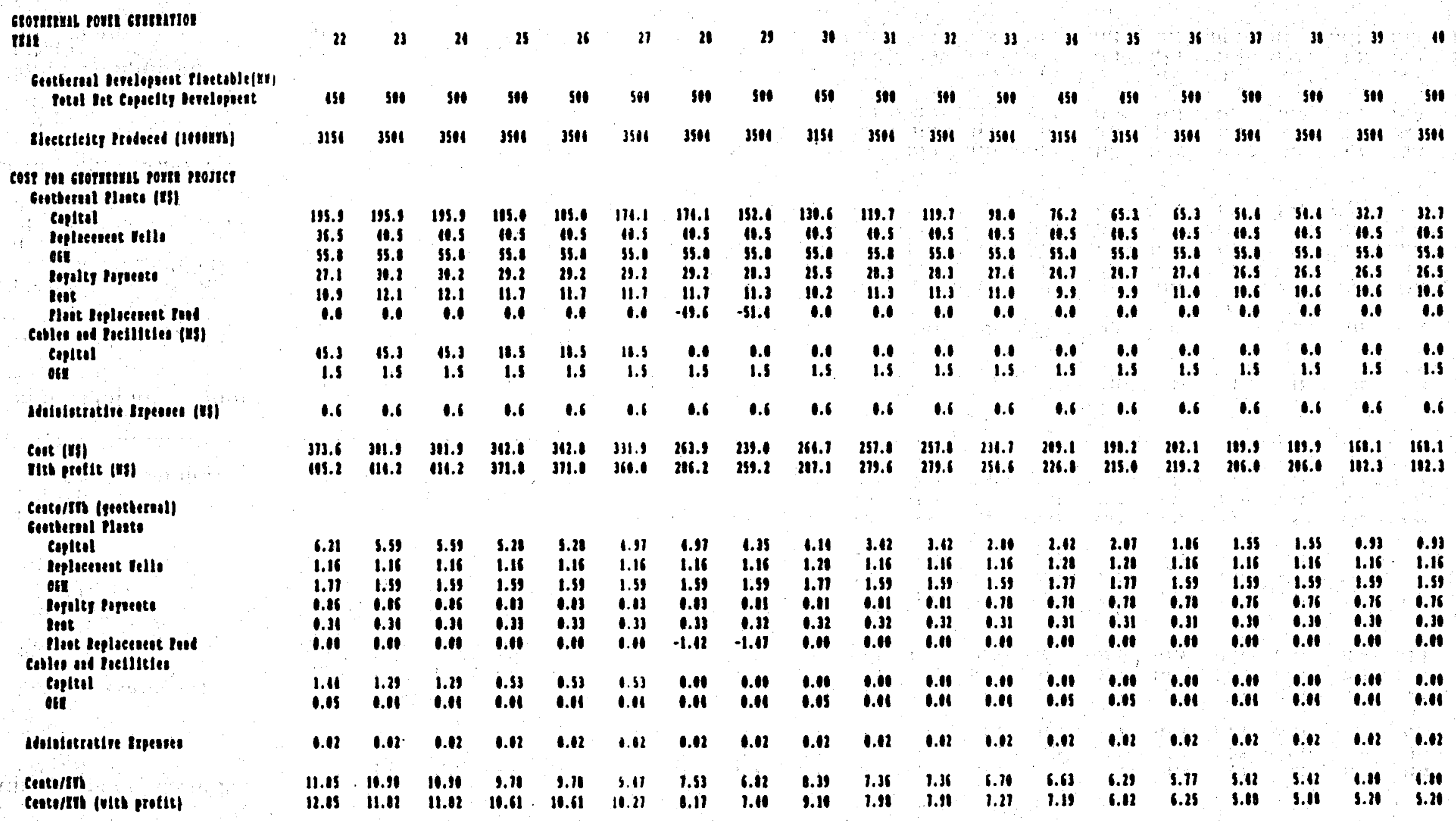




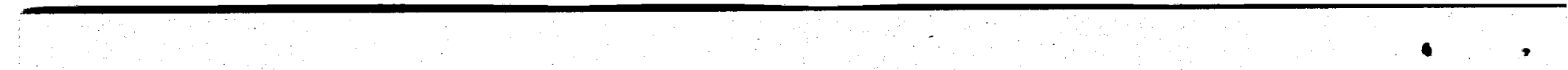


SCENARIO 2

$25 \mathrm{MW}$ power plants using

high plant/wellfield costs and a

$20 \%$ contingency 


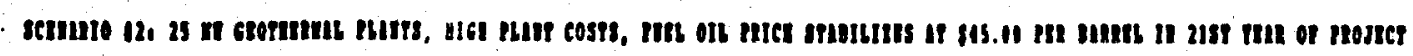

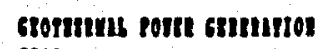

\begin{tabular}{|c|c|c|c|c|c|c|c|c|c|c|c|c|c|c|c|c|c|c|c|c|c|}
\hline min & 1 & 1 & $\mathbf{3}$ & 1 & 1 & 6 & 1 & 1 & , & 11 & II & 12 & II & 11 & 15 & 16 & 11 & II & 19 & 11 & 11 \\
\hline 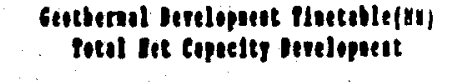 & & & $\$$ & 21 & $\begin{array}{l}25 \\
111\end{array}$ & 125 & $\begin{array}{l}25 \\
151\end{array}$ & $\begin{array}{ll}25 \\
115\end{array}$ & 211 & 225 & $\begin{aligned} 25 \\
250\end{aligned}$ & $\begin{array}{r}25 \\
275\end{array}$ & $\begin{aligned} 25 \\
301\end{aligned}$ & $\begin{array}{r}25 \\
325\end{array}$ & $\begin{array}{l}25 \\
350\end{array}$ & $\begin{array}{r}25 \\
375\end{array}$ & 25 & 15 & $\begin{array}{l}25 \\
\text { ist }\end{array}$ & 28 & $\begin{array}{l}25 \\
\text { sin }\end{array}$ \\
\hline Electrjelty feoloeed (1010ir)] & & & 351. & 326 & $m 1$ & 816 & I1S1 & 1226 & 11112 & $15 n$ & 1752 & 1911 & 1112 & 2211 & 2133 & 2621 & 2101 & 2911 & 3154 & 1329 & JSIS \\
\hline 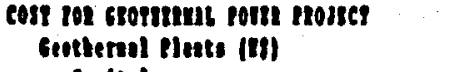 & & & & & & & & & & & & & & & & & & & & & \\
\hline $\begin{array}{l}\text { Cepleul } \\
\text { eploenent Tello }\end{array}$ & 26.1 & 26.1 & 32.3 & 32.3 & 311.1 & $\begin{array}{l}71.1 \\
5.1\end{array}$ & $\begin{array}{r}111.5 \\
11.1\end{array}$ & $\begin{array}{r}111.5 \\
11.1\end{array}$ & $\begin{array}{c}114.7 \\
16.2\end{array}$ & $\begin{array}{c}134.1 \\
16.2\end{array}$ & $\begin{array}{l}136.1 \\
11.6\end{array}$ & $\begin{array}{l}156.8 \\
11.6\end{array}$ & $\begin{array}{r}112.9 \\
21.1\end{array}$ & $\begin{array}{r}112.9 \\
21.0\end{array}$ & $\begin{array}{r}219.1 \\
32.1\end{array}$ & $\begin{array}{r}219.1 \\
32.1\end{array}$ & $\begin{array}{r}231.2 \\
31.1\end{array}$ & $\begin{array}{r}235.2 \\
11.1\end{array}$ & $\begin{array}{r}261.3 \\
13.2\end{array}$ & $\begin{array}{r}261.3 \\
11.2\end{array}$ & $\begin{array}{r}261.3 \\
11.6\end{array}$ \\
\hline $\begin{array}{l}\text { ofI } \\
\text { Iogulty Iipente }\end{array}$ & & & 9.6 & 11.1 & 18.2 & 21.1 & 31.1 & 33.1 & 31.8 & 13.3 & $\begin{array}{l}11.1 \\
12.6\end{array}$ & $\begin{array}{l}32.9 \\
11.2\end{array}$ & 51.1 & 62.5 & 61.3 & $n 2.2$ & 11.6 & 11.1 & 16.6 & 91.1 & 96.2 \\
\hline $\begin{array}{l}\text { Iopulty lipants } \\
\text { leet }\end{array}$ & & & & 1.1 & & & & & & & 12.6 & 11.2 & 15.5 & 11.8 & 19.1 & 11.1 & 21.1 & 25.0 & 21.3 & 29.1 & 31.2 \\
\hline 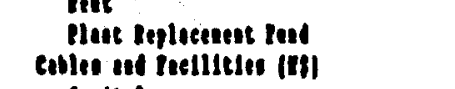 & 1.0 & 1.4 & 156.0 & $\begin{array}{l}1.0 \\
1.0\end{array}$ & $\begin{array}{l}1.3 \\
139.1\end{array}$ & $\begin{array}{l}2.1 \\
1.1\end{array}$ & 131.3 & 1.6 & $\begin{array}{l}3.8 \\
121.5\end{array}$ & 1.4 & 10.1 & $\begin{array}{l}3.1 \\
1.1\end{array}$ & 9.2 & $\begin{array}{l}1.8 \\
1.1\end{array}$ & $\begin{array}{r}7.8 \\
86.1\end{array}$ & $\begin{array}{l}3.1 \\
0.1\end{array}$ & $\begin{array}{r}9.2 \\
11.3\end{array}$ & $\begin{array}{l}11.0 \\
1.6\end{array}$ & $\begin{array}{l}10.9 \\
61.5\end{array}$ & $\begin{array}{l}11.8 \\
1.1\end{array}$ & $\begin{array}{l}12.1 \\
11.1\end{array}$ \\
\hline tupltel & 10.3 & 11.8 & $\begin{array}{r}11.3 \\
1.1\end{array}$ & 11.0 & 18.1 & $\begin{array}{l}11.8 \\
1.5\end{array}$ & $\mathbf{6 1 . 6}$ & 11.9 & $\begin{array}{c}61.4 \\
1.5\end{array}$ & $\begin{array}{l}6.1 \\
1.5\end{array}$ & $\begin{array}{l}6.0 \\
1.5\end{array}$ & 61.1 & 11.4 & $\begin{array}{l}61.0 \\
1.9\end{array}$ & $\begin{array}{l}68.1 \\
1.5\end{array}$ & 61.0 & 18.1 & 11.4 & $\mathbf{6 1 . 1}$ & 11.4 & $\begin{array}{l}18.1 \\
1.5\end{array}$ \\
\hline Inlatotratin Bxpence (If) & 1.1 & 1.1 & 0.1 & 0.1 & 1.1 & 6.1 & 1.2 & 1.1 & 4.2 & 1.3 & 1.3 & 6.1 & 1.1 & 0.1 & 1.1 & e.5 & 1.5 & 1.5 & 0.5 & 0.6 & 0.6 \\
\hline $\begin{array}{l}\text { leit (x) } \\
\text { ints profit (ng) }\end{array}$ & b1.1 & $\begin{array}{l}61.5 \\
11.2\end{array}$ & $\begin{array}{l}211.1 \\
21.1\end{array}$ & $\begin{array}{l}131.1 \\
111.8\end{array}$ & $\begin{array}{l}313.1 \\
319.8\end{array}$ & $\begin{array}{l}119.8 \\
195.1\end{array}$ & $\begin{array}{l}351.1 \\
319.1\end{array}$ & $\begin{array}{l}212.3 \\
211.1\end{array}$ & $\begin{array}{l}311.1 \\
112.5\end{array}$ & $\begin{array}{l}211.3 \\
216.1\end{array}$ & $\begin{array}{l}113.6 \\
\text { is1.1 }\end{array}$ & 321.1 & $\begin{array}{l}151.1 \\
193.8\end{array}$ & $\begin{array}{l}366.5 \\
391.5\end{array}$ & $\begin{array}{l}191.6 \\
333.2\end{array}$ & $\begin{array}{l}113.1 \\
111.0\end{array}$ & $\begin{array}{l}316.3 \\
371.1\end{array}$ & $\begin{array}{l}139.1 \\
198.1\end{array}$ & $\begin{array}{l}561.3 \\
611.3\end{array}$ & $\begin{array}{l}501.6 \\
350.5\end{array}$ & $\begin{array}{l}319.2 \\
611.3\end{array}$ \\
\hline 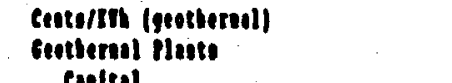 & & & & & & & & & & & & & & & & & & & & & \\
\hline $\begin{array}{l}\text { Coplen } \\
\text { Poplisenest bello }\end{array}$ & 1.16 & 1.16 & 11.11 & 9.91 & $\begin{array}{l}11.11 \\
1.11\end{array}$ & $\begin{array}{l}1.95 \\
1.62\end{array}$ & $\begin{array}{l}1.91 \\
1.63\end{array}$ & $\begin{array}{l}1.52 \\
1.11\end{array}$ & $\begin{array}{l}9.31 \\
1.16\end{array}$ & $\begin{array}{l}1.29 \\
1.61\end{array}$ & $\begin{array}{l}1.15 \\
1.21\end{array}$ & $\begin{array}{l}1.14 \\
1.12\end{array}$ & $\begin{array}{l}0.10 \\
1.21\end{array}$ & $\begin{array}{l}1.13 \\
1.11\end{array}$ & $\begin{array}{l}8.52 \\
1.32\end{array}$ & $\begin{array}{l}1.95 \\
1.23\end{array}$ & $\begin{array}{l}8.39 \\
1.13\end{array}$ & $\begin{array}{l}1.21 \\
1.21\end{array}$ & $\begin{array}{l}1.29 \\
1.11\end{array}$ & $\begin{array}{l}1.14 \\
1.31\end{array}$ & $\begin{array}{l}1.16 \\
1.39\end{array}$ \\
\hline ofl & & & 2.75 & 2.15 & 8.11 & 1.18 & 1.18 & 2.11 & 2.75 & 2.15 & $\begin{array}{l}2.15 \\
1.12\end{array}$ & $\begin{array}{l}1.13 \\
1.11\end{array}$ & $\begin{array}{l}2.13 \\
1.11\end{array}$ & $\begin{array}{l}2.15 \\
0.16\end{array}$ & 2.15 & $\begin{array}{l}2.75 \\
1.11\end{array}$ & $\begin{array}{l}2.11 \\
1.11\end{array}$ & $\begin{array}{l}2.18 \\
1.11\end{array}$ & $\begin{array}{l}2.15 \\
1.11\end{array}$ & $\begin{array}{l}2.78 \\
1.89\end{array}$ & $\begin{array}{l}1.15 \\
0.06\end{array}$ \\
\hline 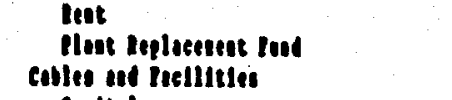 & 0.17 & $\begin{array}{l}1.21 \\
. .11\end{array}$ & 11.21 & $\begin{array}{l}0.11 \\
1.11\end{array}$ & $\begin{array}{l}1.21 \\
19.11\end{array}$ & 1.21 & $\begin{array}{r}0.21 \\
12.61\end{array}$ & $\begin{array}{l}1.29 \\
1.11\end{array}$ & $\begin{array}{l}0.21 \\
1.61\end{array}$ & $\begin{array}{l}0.11 \\
0.11\end{array}$ & $\begin{array}{l}0.29 \\
6.26\end{array}$ & 0.11 & $\begin{array}{l}9.29 \\
1.66\end{array}$ & 0.111 & 3.31 & $\begin{array}{l}1.32 \\
0.11\end{array}$ & $\begin{array}{l}0.33 \\
2.65\end{array}$ & 0.11 & $\begin{array}{l}1.11 \\
1.81\end{array}$ & 0.36 & 0.31 \\
\hline Mintal & 11.51 & 11.51 & $\begin{array}{l}11.51 \\
1.21\end{array}$ & $\begin{array}{r}12.11 \\
0.11\end{array}$ & $\begin{array}{l}9.11 \\
1.11\end{array}$ & $\begin{array}{l}1.16 \\
0.11\end{array}$ & $\begin{array}{l}6.11 \\
1.11\end{array}$ & $\begin{array}{l}3.55 \\
1.12\end{array}$ & $\begin{array}{l}1.85 \\
1.11\end{array}$ & $\begin{array}{l}1.31 \\
1.11\end{array}$ & $\begin{array}{l}3.11 \\
. .19\end{array}$ & $\begin{array}{l}3.53 \\
1.11\end{array}$ & $\begin{array}{l}3.23 \\
0.11\end{array}$ & $\begin{array}{l}2.99 \\
1.01\end{array}$ & $\begin{array}{l}2.11 \\
1.16\end{array}$ & $\begin{array}{l}2.53 \\
\text { c.16 }\end{array}$ & $\begin{array}{l}2.11 \\
6.15\end{array}$ & $\begin{array}{l}2.11 \\
0.05\end{array}$ & $\begin{array}{l}2.16 \\
0.05\end{array}$ & $\begin{array}{l}2.11 \\
0.41\end{array}$ & $\begin{array}{l}1.91 \\
1.11\end{array}$ \\
\hline Intolotratloe Inpents & 0.11 & 1.02 & 9.12 & e.n & 1.12 & 0.12 & 1.01 & 1.12 & 0.12 & 0.11 & 0.12 & 0.12 & 1.02 & e.11 & 1.02 & 1.02 & 0.12 & 0.01 & 0.12 & 0.01 & 1.12 \\
\hline 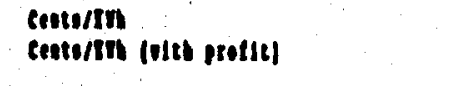 & $\begin{array}{l}19.25 \\
21.18\end{array}$ & $\begin{array}{l}19.11 \\
20.11\end{array}$ & $\begin{array}{l}11.11 \\
61.11\end{array}$ & $\begin{array}{l}\mathbf{2 6 . 1 1} \\
\mathbf{3 . 3 2}\end{array}$ & $\begin{array}{l}11.11 \\
11.91\end{array}$ & $\begin{array}{l}20.51 \\
22.21\end{array}$ & $\begin{array}{l}31.31 \\
36.12\end{array}$ & $\begin{array}{l}19.13 \\
18.16\end{array}$ & $\begin{array}{l}21.11 \\
21.11\end{array}$ & $\begin{array}{l}11.76 \\
11.11\end{array}$ & $\begin{array}{l}21.11 \\
26.11\end{array}$ & $\begin{array}{l}16.16 \\
11.61\end{array}$ & $\begin{array}{l}21.11 \\
23.51\end{array}$ & $\begin{array}{l}16.19 \\
11.15\end{array}$ & $\begin{array}{l}21.11 \\
21.11\end{array}$ & $\begin{array}{l}15.12 \\
11.05\end{array}$ & $\begin{array}{l}11.11 \\
21.36\end{array}$ & $\begin{array}{l}15.11 \\
11.11\end{array}$ & $\begin{array}{l}11.11 \\
19.11\end{array}$ & $\begin{array}{l}15.25 \\
16.51\end{array}$ & $\begin{array}{l}16.21 \\
11.62\end{array}$ \\
\hline
\end{tabular}


sernitu is coss.

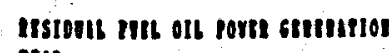

IIII

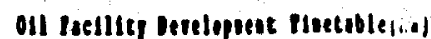

intal let concity Benelopuest

Oll Prles (f thI)

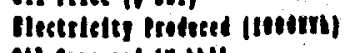

ill conmed |f $\mathrm{WI}$ |

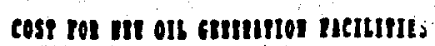

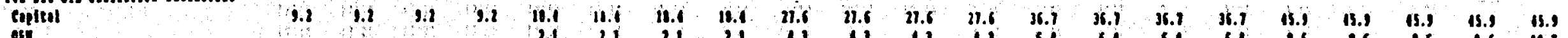

oir

Ivel

Itelolotrotire mposes (as)

cont (IS)

IIt) freflt (II)

cetoim (oII)

tepltel

of:

dinistutive Ineseses

Potol ceotorm

Potel teutunn vith protit

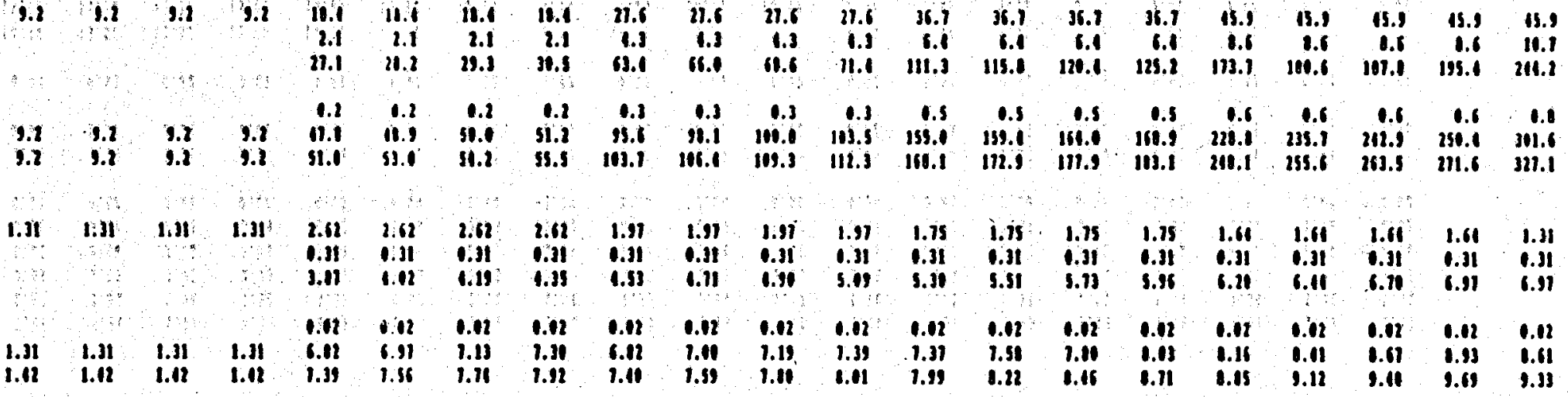

solisnestsut int oft poste strents. mil

Solar/oul berelopunt efeetulle(vi) fotal let espetty levelopeset

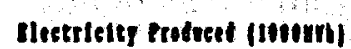

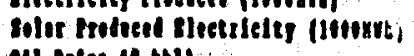

oil trien |f bil|

oll isshest Blectrlesty (10mm)

oll cosmen (L III)

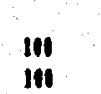

111 $111 \quad 1010 \quad 111$

$15.1126 .1121 .11 \quad 21.12 \quad 28$.

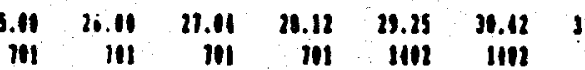

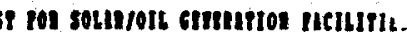

Capltal

ofl

rut

Ilotalitmetre Inpewes (n)

Cont (n)

itt irollt (II)

\section{cestoirm \\ enteul \\ oft}

Intelotratire Inences

intal entoirm

fotel coetentit its profle

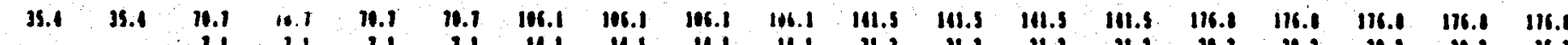

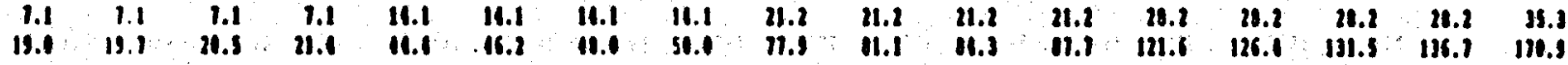

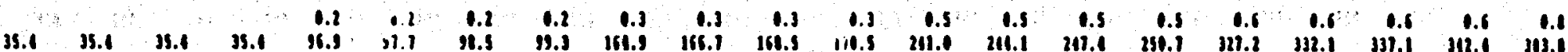

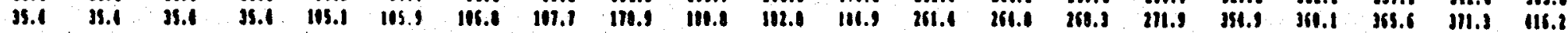

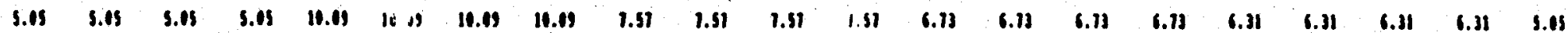

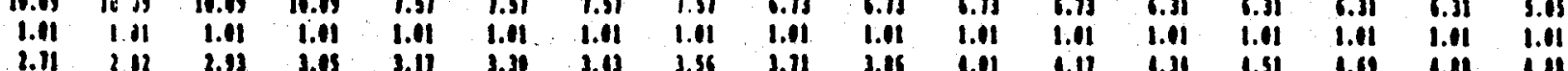

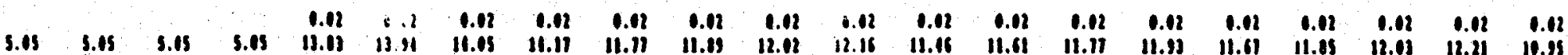

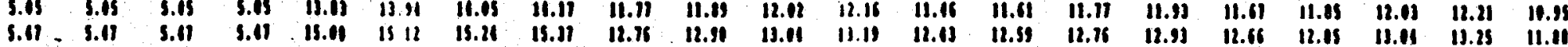


senimo is cons.

\begin{tabular}{|c|c|c|c|c|c|c|c|c|c|c|c|c|c|c|c|c|c|c|c|}
\hline Im! & $u$ & 23 & 11 & 25 & 3 & $\|$ & $m$ & 29 & 3 & JI & 32 & 33 & 34 & 35 & 36 & נ1 & "! & 39 & 4 \\
\hline 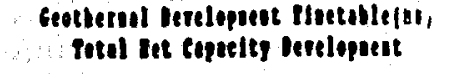 & ISI & 811 & Sil & 310 & Sil & $\$ 00$ & 3110 & mil & Ist & sin & 501. & 310 & (SSO & (5) & se1 & 311 & 510 & 311 & 10 \\
\hline 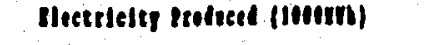 & JIS! & 3311 & 3311 & 3391 & 3811 & 3501 & 3391 & 3501 & JISI & 3811 & 394 & 3511 & 3151 & 3ist & 3811 & 3511 & 381 & 3511 & JSII \\
\hline 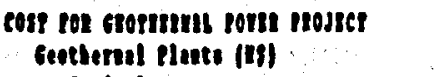 & & & & & & & & & & & & & & & & & & & \\
\hline & 233.2 & 235.2 & 233.2 & 222.1 & $2 m .1$ & 209.1 & vg.1 & 112.9 & 156.1 & 113.1 & 113.1 & 117.6 & 21.5 & 11.1 & 11.1 & 65.3 & 01.3 & 39.2 & 31.2 \\
\hline ent fllle & $\begin{array}{l}18.6 \\
96.2\end{array}$ & $\begin{array}{l}11.0 \\
11.2\end{array}$ & $\begin{array}{l}51.0 \\
8.2\end{array}$ & $\begin{array}{l}11.0 \\
16.2\end{array}$ & $\begin{array}{l}31.1 \\
x .2\end{array}$ & $\begin{array}{l}51.0 \\
96.2\end{array}$ & 11.8 & 31.1 & 91.1 & $\begin{array}{l}31.1 \\
8.2\end{array}$ & $\begin{array}{l}51.0 \\
9.2\end{array}$ & 31.0 & & 31.0 & s1., & & si.4, & 31.0 & 31.0 \\
\hline Rogiley Popenses & ili. & 31.2 & 30.2 & 29.2 & 29.1 & 20.2 & 3.2 & $2 i .1$ & 25.5 & ii.j & 20.3 & 21.1 & 26.1 & $\begin{array}{l}18.2 \\
11.1\end{array}$ & $\begin{array}{l}3.2 \\
21.1\end{array}$ & $\begin{array}{l}8.2 \\
6.5\end{array}$ & $\begin{array}{l}3 . .2 \\
26.5\end{array}$ & $\begin{array}{l}11.2 \\
11.5\end{array}$ & $\begin{array}{l}20.8 \\
26.5\end{array}$ \\
\hline & 11..9 & 11.1 & 12.1 & 11.1 & 11.1 & 11.1 & 11.1 & 11.8 & 10.2 & 11.3 & 11.3 & 11.0 & 9.9 & 9.9 & 11.8 & 11.6 & 10.6 & 11.6 & 11.6 \\
\hline 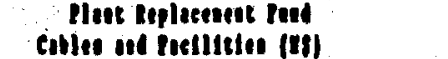 & 0.1 & 0.1 & 1.0 & 0.0 & 0.1 & 0.1 & -81.5 & -53.1 & 0.0 & 0.1 & 0.1 & 9.1 & 0.1 & 1.0 & 0.1 & 0.0 & 0.1 & 1.1 & 0.0 \\
\hline $\begin{array}{l}\text { Epitul } \\
\text { ofie }\end{array}$ & $\begin{array}{l}13.3 \\
1.3\end{array}$ & $\begin{array}{l}15.1 \\
1.5\end{array}$ & $\begin{array}{l}15.3 \\
1.5\end{array}$ & $\begin{array}{l}11.5 \\
1.5\end{array}$ & $\begin{array}{l}11.5 \\
1.5\end{array}$ & $\begin{array}{l}11.5 \\
1.5\end{array}$ & $\begin{array}{l}0.8 \\
1.5\end{array}$ & $\begin{array}{l}1.1 \\
1.1\end{array}$ & $\begin{array}{l}0.1 \\
1.5\end{array}$ & $\begin{array}{l}1.1 \\
1.5\end{array}$ & $\begin{array}{l}1.0 \\
1.5\end{array}$ & $\begin{array}{l}0.1 \\
1.5\end{array}$ & $\begin{array}{l}1.4 \\
1.5\end{array}$ & $\begin{array}{l}1.1 \\
1.5\end{array}$ & $\begin{array}{l}0.4 \\
1.5\end{array}$ & $\begin{array}{l}\text {.0 } \\
\text { i.5 }\end{array}$ & $\begin{array}{l}0.1 \\
1.5\end{array}$ & i.8 & $\begin{array}{l}0.4 \\
1.5\end{array}$ \\
\hline Indolotrutine ippenes (ns) & 0.1 & 0.1 & 0.6 & 0.6 & 0.6 & 0.6 & 0.6 & 0.6 & 1.6 & 0.6 & 0.6 & 1.6 & 0.6 & 0.6 & 0.6 & 0.6 & 0.6 & 0.6 & 0.6 \\
\hline 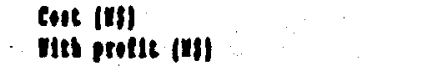 & $\begin{array}{l}\cos .1 \\
301.1\end{array}$ & $\begin{array}{l}115.1 \\
315.2\end{array}$ & $\begin{array}{l}313.4 \\
319.2\end{array}$ & $\begin{array}{l}333.8 \\
171.9\end{array}$ & $\begin{array}{l}0131.4 \\
111.5\end{array}$ & $\begin{array}{l}212.1 \\
656.3\end{array}$ & 311.1 & 315.2 & 311.8 & 335.1 & 315.1 & 301.3 & 211.3 & 263.2 & 39.1 & 231.1 & 251.1 & 221.6 & 211.6 \\
\hline cente/mn (geothernal) & & & & & & & & & & & $m .1$ & 384.3 & SH.8 & 80.1 & 9.6 & 211.2 & $\mathbf{3 1 . 2}$ & 811.8 & 211.9 \\
\hline Gorlet & & & & & & & & & & & & & & & & & & & \\
\hline & $\begin{array}{l}1.16 \\
1.31\end{array}$ & l.n & $\begin{array}{l}6.11 \\
1.51\end{array}$ & b.34 & 6.11 & 3.31 & 3.91 & 3.23 & (.91 & 1.11 & 1.11 & 3.16 & 2.90 & 2.19 & 2.21 & 1.11 & 1.16 & 1.12 & 1.12 \\
\hline lellis & & & $\begin{array}{l}1.51 \\
2.11\end{array}$ & & & $\begin{array}{l}1.51 \\
2.15\end{array}$ & $\begin{array}{l}1.51 \\
2.15\end{array}$ & 1.18 & $\begin{array}{l}1.71 \\
3.18\end{array}$ & $\begin{array}{l}1.51 \\
2.15\end{array}$ & $\begin{array}{l}1.51 \\
2.15\end{array}$ & 1.51 & 1.1 & 1.11 & & & 1.51 & 1.51 & 1.51 \\
\hline & 0.16 & 2.11 & 2.13 & 2.13 & 2.13 & 2.73 & 2.13 & 2.13 & 3.18 & 2.13 & 2.15 & 2.19 & 3.15 & 3.01 & 2.15 & 2.15 & 2.15 & 2.15 & 2.15 \\
\hline Eopulty tipences & 0.16 & 0.16 & 0.16 & 0.13 & .11 & 1.11 & 0.13 & 0.11 & 0.11 & 1.11 & 0.81 & 0.16 & 0.11 & 1.11 & 1.11 & 0.16 & 9.11 & $\therefore 16$ & 0.16 \\
\hline Iixt & 0.31 & O.11 & 13 & 0.33 & e.j3 & 0.33 & 43 & .32 & 0.32 & 0.32 & 1.32 & e.3t & O.J1 & 0.31 & ..3t & .31 & 9.110 & 0.31 & (.31 \\
\hline 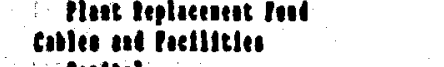 & 1.11 & 0.10 & 1.10 & 0.11 & 0.01 & 1.10 & -1.61 & -1.11 & 0.11 & 1.01 & 1.10 & 1.11 & 1.01 & 1.91 & 1.11 & 0.11 & 1.01 & 1.01 & 0.11 \\
\hline offer & $\begin{array}{l}1.11 \\
1.11\end{array}$ & $\begin{array}{l}1.29 \\
0.11\end{array}$ & $\begin{array}{l}1.29 \\
1.11\end{array}$ & 0.31 & 1.53 & (..5) & 1.10 & 0.11 & 0.10 & 0.11 & 0.11 & 0.11 & 0.11 & 0.110 & 0.11 & 0.11 & 0.11 & 0.11 & 0.11 \\
\hline Mninlestrtine spenon & & & & & & & & & & & & & & & & & & & \\
\hline & & & . & & & 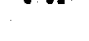 & 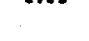 & . & . & 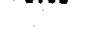 & 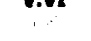 & 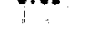 & 0.06 & 0. & 0.11 & 0.04 & $1 . n$ & 0.08 & $0 . n$ \\
\hline & & & & & & & & & & & & & & & $1.8-5-x+1$ & & & 6.32 & 6.31 \\
\hline & & $.7 n$ & 10.11 & .13 & $\mathbf{3 . 1 3}$ & 13.12 & 18.61 & $9 . x$ & 11.06 & 20.33 & 10.39 & 3.11 & 8.51 & 9.12 & 1.J3 & 1.11 & 1.11 & 1.11 & 11 \\
\hline
\end{tabular}


semurre 12 corr.

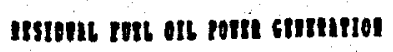

IIII

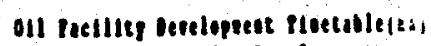
Qutul let Coputty Derelopest

QII irlee 18 wil

Bleetrielty Prolueed (mominn)

oll consened (1) inll)

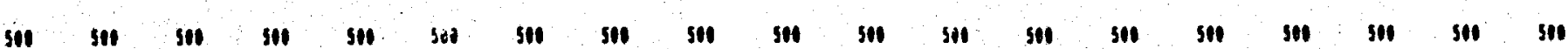

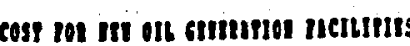

eapltel
oft
lut

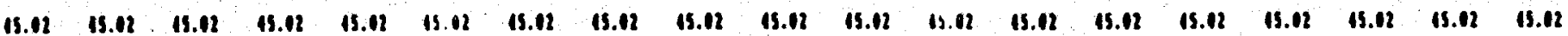

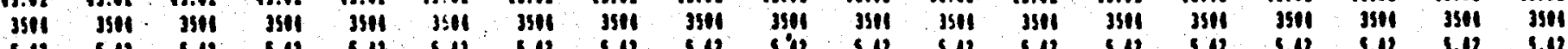

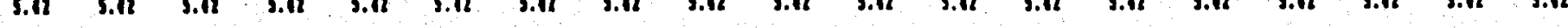

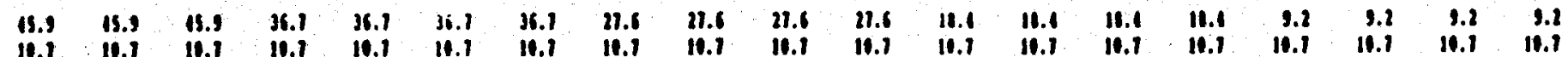

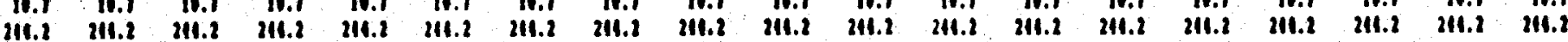

Intolstratin Itpenics (IS) Coit (ns)

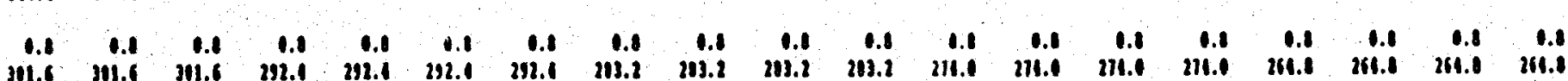
IIth Protit (II)

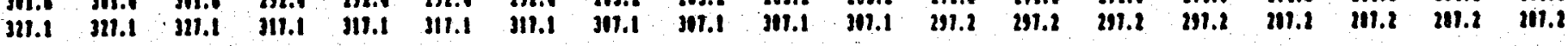

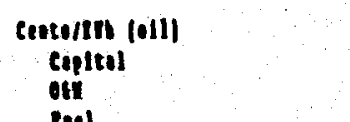

(net

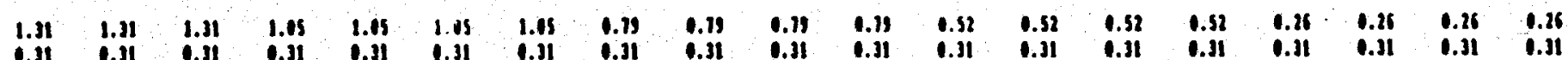

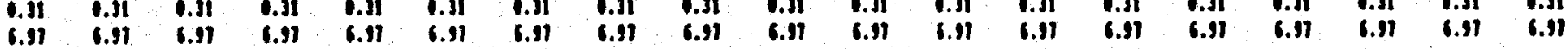

Intolitratse inpences Potal tenturtn fotal eneteitm ilt protit

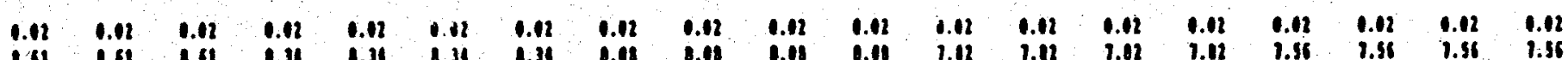

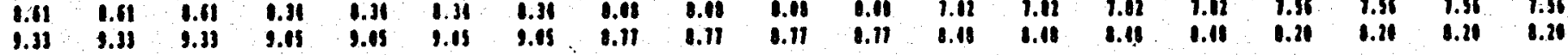

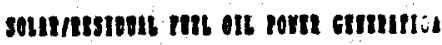
min

Solsr/oil bevelopuent Flatable(urj pesil let enecte Perelopeent

Eletriefty enoleed flowmy

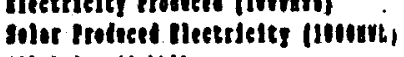
oll Prlee 18 bill

ofl Prodiced lleetrielty (100umb)

oit conemed (I HII)

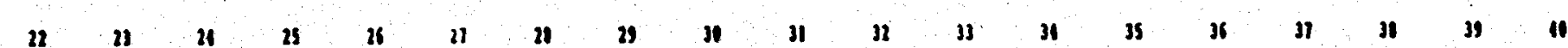

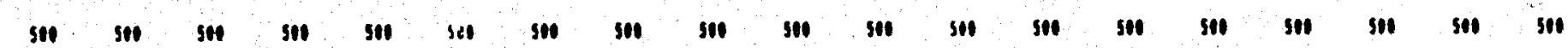

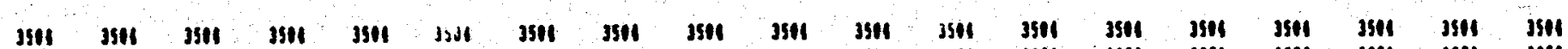

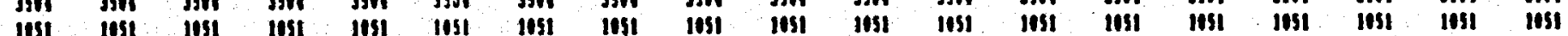

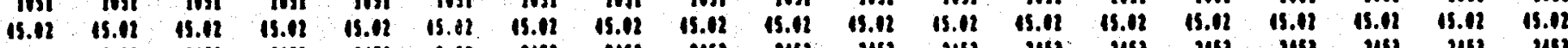

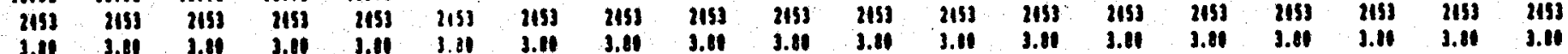

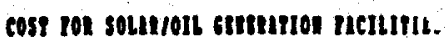

\begin{tabular}{|c|c|c|c|c|c|c|c|c|c|c|c|c|c|c|c|c|c|c|c|}
\hline $\begin{array}{l}\text { Enptul } \\
\text { oft } \\
\text { Poil }\end{array}$ & $\begin{array}{l}176.8 \\
35.3 \\
171.9\end{array}$ & $\begin{aligned} 116.8 \\
31.3 \\
111.9\end{aligned}$ & $\begin{array}{l}176.1 \\
35.3 \\
171.9\end{array}$ & $\begin{array}{l}111.5 \\
11.3 \\
171.5\end{array}$ & $\begin{array}{l}111.5 \\
15.3 \\
171.5\end{array}$ & $\begin{array}{r}111.5 \\
351 \\
178.8\end{array}$ & $\begin{array}{r}111.3 \\
33.3 \\
111.3\end{array}$ & $\begin{array}{r}106.1 \\
35.3 \\
131.5\end{array}$ & $\begin{array}{r}166.1 \\
35.3 \\
171.9\end{array}$ & $\begin{array}{l}106.1 \\
31.1 \\
111.5\end{array}$ & $\begin{array}{l}116.1 \\
35.3 \\
171.9\end{array}$ & $\begin{array}{r}10.1 \\
35.1 \\
110.1\end{array}$ & $\begin{array}{l}11.1 \\
35.3 \\
111.9\end{array}$ & $\begin{array}{c}10.1 \\
35.3 \\
111.5\end{array}$ & $\begin{array}{l}11.1 \\
35.3 \\
111.8\end{array}$ & $\begin{array}{l}35.1 \\
35.3 \\
17.1\end{array}$ & $\begin{array}{r}35.1 \\
31.3 \\
111.5\end{array}$ & $\begin{array}{l}35.1 \\
35.1 \\
114.1\end{array}$ & $\begin{array}{r}35.1 \\
31.3 \\
111.9\end{array}$ \\
\hline 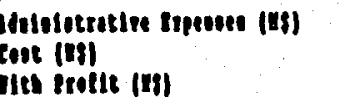 & $\begin{array}{l}1.8 \\
313.8 \\
116.2\end{array}$ & $\begin{array}{l}1.8 \\
313.1 \\
116.2\end{array}$ & $\begin{aligned} 0.8 \\
393.1 \\
116.2\end{aligned}$ & $\begin{array}{l}1.1 \\
311.4 \\
311.9\end{array}$ & $\begin{array}{l}1.1 \\
312.1 \\
311.1\end{array}$ & $\begin{array}{l}11 \\
311.1 \\
311.1\end{array}$ & $\begin{array}{l}1.1 \\
311.1 \\
311.9\end{array}$ & $\begin{array}{l}1.8 \\
313.0 \\
339.8\end{array}$ & $\begin{array}{l}1.4 \\
313.4 \\
139.3\end{array}$ & $\begin{array}{r}1.1 \\
313.1 \\
334.5\end{array}$ & $\begin{array}{r}1.8 \\
313.4 \\
339.5\end{array}$ & $\begin{array}{r}1.1 \\
211.1 \\
301.1\end{array}$ & $\begin{aligned} 1.8 \\
211.1 \\
311.2\end{aligned}$ & $\begin{aligned} 1.8 \\
2 m .1 \\
311.2\end{aligned}$ & $\begin{array}{l}1.1 \\
211.1 \\
311.2\end{array}$ & $\begin{array}{r}1.8 \\
212.8 \\
262.8\end{array}$ & $\begin{array}{l}1.1 \\
212.3 \\
112.1\end{array}$ & $\begin{array}{r}0.1 \\
212.3 \\
262.8\end{array}$ & $\begin{array}{l}1.1 \\
212.3 \\
211.0\end{array}$ \\
\hline $\begin{array}{l}\text { Itornm } \\
\text { Cepitul } \\
\text { ofi } \\
\text { foel }\end{array}$ & $\begin{array}{l}9.11 \\
1.11 \\
1.11\end{array}$ & $\begin{array}{l}3.05 \\
1.01 \\
1.11\end{array}$ & $\begin{array}{l}3.15 \\
1.11 \\
1.11\end{array}$ & $\begin{array}{l}1.11 \\
1.11 \\
1.11\end{array}$ & $\begin{array}{l}1.11 \\
1.01 \\
1.11\end{array}$ & $\begin{array}{l}1.11 \\
1.11 \\
1.11\end{array}$ & $\begin{array}{l}1.11 \\
1.01 \\
1.01\end{array}$ & $\begin{array}{l}1.63 \\
1.11 \\
1.81\end{array}$ & $\begin{array}{l}3.01 \\
1.01 \\
1.01\end{array}$ & $\begin{array}{l}1.11 \\
1.01 \\
1.11\end{array}$ & $\begin{array}{l}3.01 \\
1.01 \\
1.11\end{array}$ & $\begin{array}{l}2.11 \\
1.11 \\
1.11\end{array}$ & $\begin{array}{l}1.12 \\
1.11 \\
1.11\end{array}$ & $\begin{array}{l}2.02 \\
1.01 \\
1.11\end{array}$ & $\begin{array}{l}1.02 \\
1.11 \\
1.11\end{array}$ & $\begin{array}{l}1.01 \\
1.01 \\
1.11\end{array}$ & $\begin{array}{l}1.01 \\
1.01 \\
1.11\end{array}$ & $\begin{array}{l}1.11 \\
1.01 \\
1.01\end{array}$ & $\begin{array}{l}1.01 \\
1.01 \\
1.01\end{array}$ \\
\hline 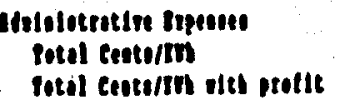 & $\begin{array}{l}1.01 \\
11.18 \\
11.11\end{array}$ & $\begin{array}{l}1.01 \\
18.95 \\
11.11\end{array}$ & $\begin{array}{l}0.12 \\
11.15 \\
11.18\end{array}$ & $\begin{array}{l}1.11 \\
9.11 \\
18.11\end{array}$ & $\begin{array}{l}1.12 \\
1.11 \\
11.11\end{array}$ & $\begin{array}{ll}1 & 1 \\
12 & 11 \\
19 & 18\end{array}$ & $\begin{array}{r}1.01 \\
9.11 \\
14.11\end{array}$ & $\begin{array}{l}1.12 \\
1.13 \\
9.11\end{array}$ & $\begin{array}{l}0.11 \\
1.13 \\
9.69\end{array}$ & $\begin{array}{l}6.12 \\
1.13 \\
.11\end{array}$ & $\begin{array}{l}1.11 \\
1.13 \\
1.69\end{array}$ & $\begin{array}{l}1.11 \\
1.11 \\
1.51\end{array}$ & $\begin{array}{l}1.02 \\
1.92 \\
8.58\end{array}$ & $\begin{array}{l}1.12 \\
1.92 \\
1.59\end{array}$ & $\begin{array}{l}1.11 \\
1.12 \\
1.51\end{array}$ & $\begin{array}{l}0.01 \\
6.11 \\
1.31\end{array}$ & $\begin{array}{l}0.12 \\
1.92 \\
1.51\end{array}$ & $\begin{array}{l}1.11 \\
6.12 \\
1.51\end{array}$ & $\begin{array}{l}1.01 \\
6.12 \\
1.51\end{array}$ \\
\hline
\end{tabular}




\section{SCENARIO 3}

$50 \mathrm{MW}$ power plants using low plant/wellfield costs and a $20 \%$ contingency 


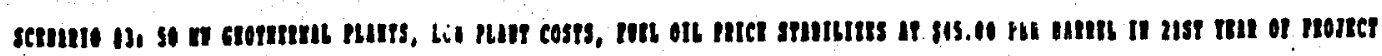

croptints port strminos

III

Seotletwil Serelopeint Plotable(II) eteil set cepeelty Derelopeest

Ilestriedty Proloed linourn)

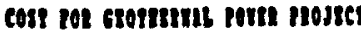

cottiral neats (II)

enitol

Peploeeme nelt:

ifI

Iopalty Bupeate

lest

thot Eeplocesent Ine

Cobles und bucilitise (II) Capitul

Inlofotratine Bpenes (19)

Cout (II)

IIt protst (n)

Centerin (geothersul)

Ceollerni Hinte

cipltil

Eepluepest tells

oin

Emplty Pomeato

plese teploesenes lont

cablis ent Patlletes

caltel

ocit

Infulotutin impane

cetertm

cotolrn (vit) protit)

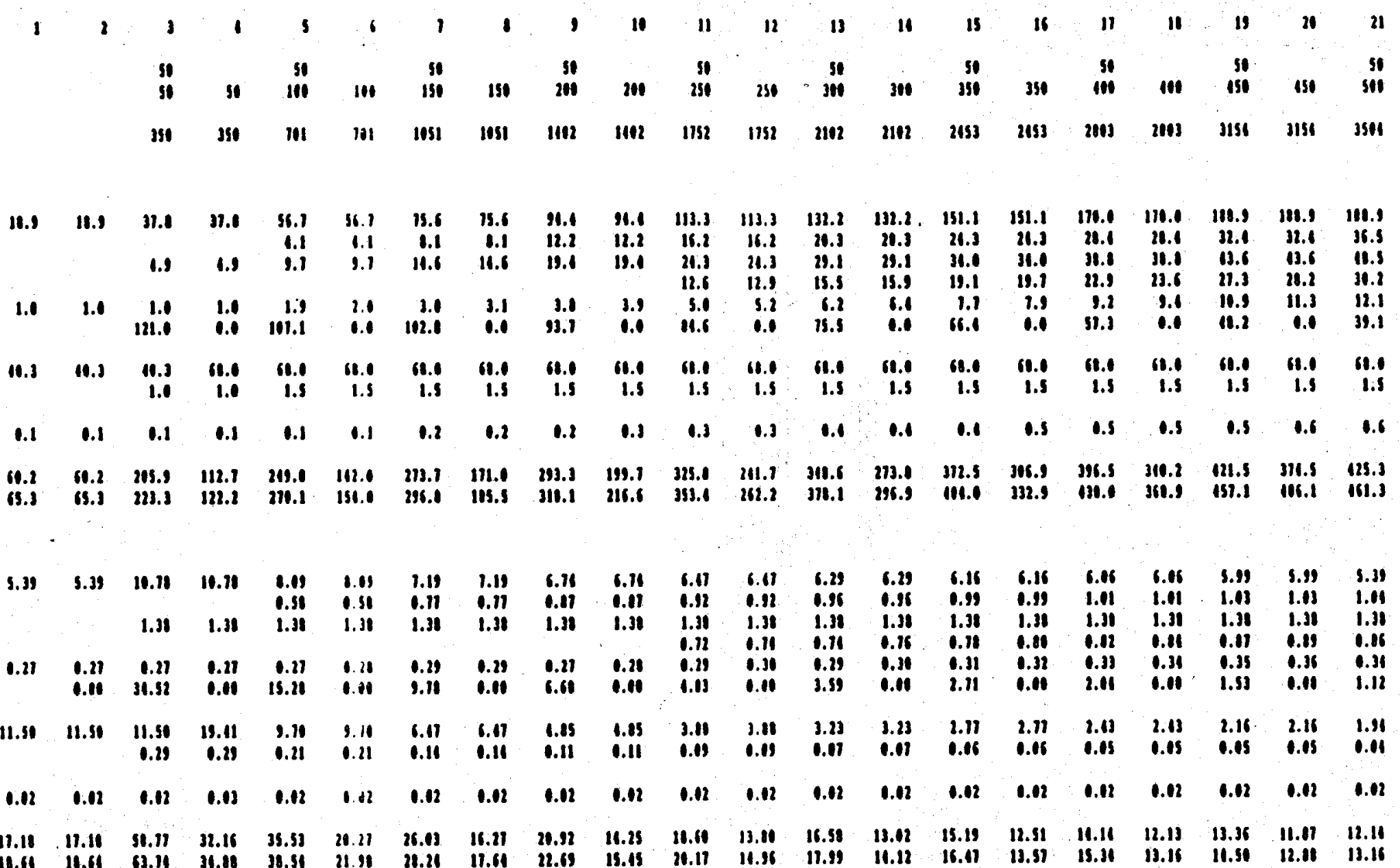


steinto is cont.

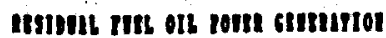

min

OII rectlity berelopent platuble(11) Potil ift encelty terelopmet

011 pelee (I wi)

teetelefts Irohest (110im)

ili commel |l bil|

cosf wot it on grmurios theturnts

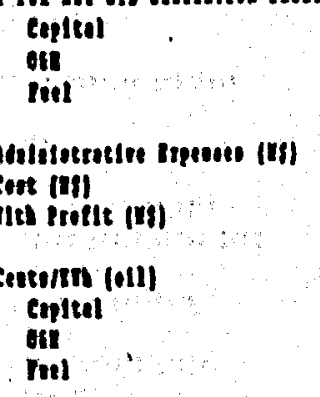

Inilitratine sipenes

Sotul entoirm

Potel cente/tn itt protlt

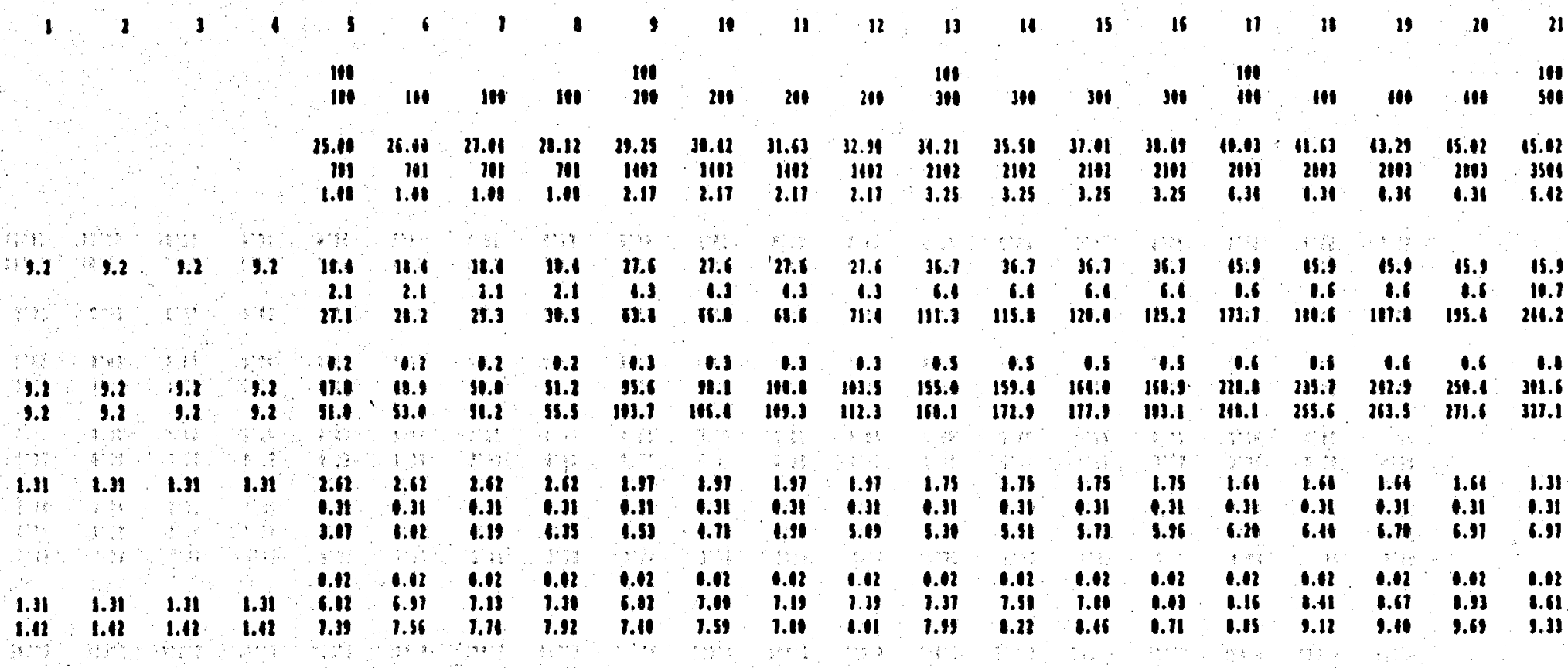

cosu/nesous ros oft eorth ctmurna min

solar/oll nemelopacit floetable(iv)

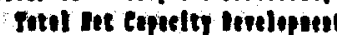

Dieetrlelty trodeet (100mm)

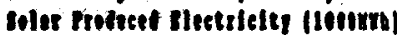

oll trlee is bil!

oll froherl Blectrielty (100em)

oll consuel (1) BHI)

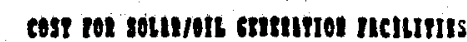

$$
\begin{aligned}
& \text { cepseal } \\
& \text { in }
\end{aligned}
$$

Molustritde Enense

Posul enturnn

potal centerm eits peotic 
Serunio is corf.

crornume rom semurtos

III

fentherul developest Plottable(z) petil iet captetty lerelopuest

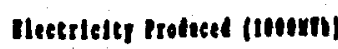

cost pes storstrmis com moster Geotherni tlents (In)

copltal

Peflecesest tells

orit

Poralty Papents

lems

Hont eploetnest ind

calles in tuellition (4) Ciples

ont

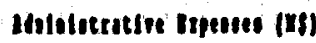

ceit (III)

sits protit (ns)

cestoinn (fenttenal)

entlersil fluts

(ipltul

Ieplecentit vell

ati

Eoplty lopente

leas

Plent Inplacenets Int

cables ane facllitles

cuptent

ons

Intolotentive Itpestee

Centosini

cintulen intt piotit)

\begin{tabular}{|c|c|c|c|c|c|c|c|c|c|c|c|c|c|c|c|c|c|}
\hline 12 & 23 & " & 38 & w & 11 & $\mathbf{2 1}$ & 23 & 30 & $\mathbf{H}$ & $\boldsymbol{3 2}$ & ${ }^{3}$ & 31 & 435 & 36 & $\mathbf{~}$ & & \\
\hline ISI & 150 & 311 & sil & st1 & Sold & 510 & ste & ISA & A51 & 511 & 511 & 150 & 100 & 151 & sin & SII & 510 \\
\hline 315 & JISI & 3511 & JSES & 35111 & 3311 & J316 & 3501 & HSI & 315 & 3501 & JSUI & 3131 & 2111 & 3158 & 3511 & 3501 & 3510 \\
\hline
\end{tabular}

\begin{tabular}{|c|c|c|c|c|c|c|c|c|c|c|c|c|c|c|c|c|c|c|}
\hline $\begin{array}{r}111.0 \\
36.5\end{array}$ & $\begin{array}{r}171.9 \\
11.5\end{array}$ & $\begin{array}{r}171.0 \\
11.5\end{array}$ & $\begin{array}{r}151.1 \\
10.5\end{array}$ & $\begin{array}{r}151.1 \\
10.5\end{array}$ & $\begin{array}{r}132.2 \\
11.5\end{array}$ & $\begin{array}{r}131.2 \\
10.5\end{array}$ & $\begin{array}{r}113.3 \\
11.5\end{array}$ & $\begin{array}{r}113.3 \\
11.5\end{array}$ & $\begin{array}{l}21.1 \\
11.5\end{array}$ & $\begin{array}{l}98.1 \\
18.5\end{array}$ & $\begin{array}{l}91.1 \\
11.5\end{array}$ & $\begin{array}{l}15.6 \\
11.5\end{array}$ & $\begin{array}{l}31.8 \\
10.5\end{array}$ & $\begin{array}{l}31.1 \\
11.5\end{array}$ & $\begin{array}{l}31.1 \\
41.5\end{array}$ & $\begin{array}{l}31.1 \\
10.5\end{array}$ & $\begin{array}{l}11.8 \\
11.5\end{array}$ & $\begin{array}{l}11.9 \\
10.5\end{array}$ \\
\hline 11.5 & 11.5 & 11.5 & 11.5 & 11.5 & 11.5 & 10.5 & 11.5 & 18.5 & 11.8 & i1.; & 11.5 & 11.5 & 11.5 & 11.5 & 11.5 & 11.5 & 18.5 & 10.8 \\
\hline 21.1 & 11.1 & 31.2 & 29.8 & 21.2 & 29.2 & 29.1 & 11.3 & 25.5 & 25.5 & 28.3 & 27.1 & 21.1 & 21.9 & 21.1 & 26.5 & 11.5 & 26.5 & 25.5 \\
\hline 11.1 & 10.8 & 12.1 & 11.1 & 11.1 & 11.1 & 11.1 & 11.3 & 11.2 & 10.2 & II.J & 11.0 & 1.1 & 0.1 & 1.9 & 10.6 & 18.6 & 11.6 & 11.6 \\
\hline 0.1 & 1.0 & 1.1 & 0.1 & 1.1 & 0.1 & -11.1 & -16.0 & 0.0 & 0.4 & e. & 1.0 & 0.1 & & & 0.1 & 0.1 & & 0.1 \\
\hline 15.3 & 15.1 & 13.3 & 11.1 & 11.5 & 11.5 & 0. & 0.1 & I. & e. & t. & I. & t. & 1. & 1. & t. & 1.0 & t. & 1.4 \\
\hline 1.5 & 1.5 & 1.8 & 1.5 & 1.5 & 1.1 & 1.5 & 1.5 & 1.8 & 1.5 & 1.5 & 1.5 & & & & 1.5 & & 1.1 & 1.5 \\
\hline 0.6 & 1.6 & 0.1 & 0.1 & 1.6 & 0.6 & 1.6 & 1.6 & 0.6 & 1.6 & 1.6 & 0.6 & 0.6 & 1.6 & 0.6 & 0.6 & 0.6 & 0.6 & 0.6 \\
\hline & 11.1 & 311.1 & 11.6 & 111.6 & 2.1 & 19.8 & H.1 & 10. & $u$ & 25 & 111.9 & & & & 1 & .1 & 117 & \\
\hline 1.2 & J11.6 & $m .1$ & mil.1 & Jn.I & 316.6 & 211.5 & 211.1 & 260.1 & 239.5 & 211.2 & 212.1 & 211.2 & I13.1 & In.2 & 101.1 & 111.1 & 159.8 & 159.5 \\
\hline
\end{tabular}

\begin{tabular}{|c|c|c|c|c|c|c|c|c|c|c|c|c|c|c|c|c|c|}
\hline $\begin{array}{l}3.31 \\
1.11 \\
1.11 \\
0.11 \\
0.11 \\
0.01\end{array}$ & $\begin{array}{l}9.39 \\
1.21 \\
1.21 \\
. .06 \\
0.11 \\
1.01\end{array}$ & $\begin{array}{l}1.11 \\
1.11 \\
1.31 \\
1.16 \\
1.31 \\
1.01\end{array}$ & $\begin{array}{l}1.31 \\
1.16 \\
1.31 \\
0.13 \\
0.31 \\
0.18\end{array}$ & $\begin{array}{l}1.31 \\
1.16 \\
1.31 \\
1.13 \\
0.31 \\
0.11\end{array}$ & $\begin{array}{l}3.11 \\
1.16 \\
1.31 \\
0.13 \\
0.31 \\
0.11\end{array}$ & $\begin{array}{c}1.11 \\
1.11 \\
1.11 \\
1.11 \\
0.11 \\
-1.21\end{array}$ & $\begin{array}{c}3.23 \\
1.16 \\
1.31 \\
1.11 \\
1.32 \\
-1.31\end{array}$ & $\begin{array}{l}3.59 \\
1.21 \\
1.51 \\
1.11 \\
. .12 \\
0.11\end{array}$ & $\begin{array}{l}2.19 \\
1.11 \\
1.51 \\
0.11 \\
0.11 \\
0.11\end{array}$ & $\begin{array}{l}2.11 \\
1.11 \\
1.11 \\
1.11 \\
1.11 \\
1.11\end{array}$ & $\begin{array}{l}2.11 \\
1.16 \\
1.11 \\
0.11 \\
1.11 \\
1.11\end{array}$ & $\begin{array}{l}2.40 \\
1.21 \\
1.51 \\
.11 \\
0.31 \\
1.11\end{array}$ & $\begin{array}{l}1.35 \\
1.11 \\
1.13 \\
0.11 \\
0.31 \\
1.11\end{array}$ & $\begin{array}{l}1.11 \\
1.21 \\
1.51 \\
. .11 \\
0.11 \\
6.11\end{array}$ & $\begin{array}{l}1.10 \\
1.16 \\
1.31 \\
1.16 \\
0.31 \\
0.11\end{array}$ & $\begin{array}{l}1.01 \\
1.11 \\
1.11 \\
0.11 \\
1.11 \\
0.11\end{array}$ & $\begin{array}{l}0.51 \\
1.16 \\
1.31 \\
1.16 \\
1.31 \\
1.10\end{array}$ \\
\hline $\begin{array}{l}1.11 \\
\text { 1.01 }\end{array}$ & $\begin{array}{l}1.11 \\
1.15\end{array}$ & $\begin{array}{l}1.21 \\
1.11\end{array}$ & $\begin{array}{l}\text { 0.53 } \\
0.11\end{array}$ & $\begin{array}{l}1.53 \\
0.11\end{array}$ & $\begin{array}{l}0.31 \\
1.01\end{array}$ & $\begin{array}{l}0.11 \\
1.11\end{array}$ & $\begin{array}{l}1.11 \\
1.11\end{array}$ & $\begin{array}{l}0.01 \\
\text { i.05 }\end{array}$ & $\begin{array}{l}1.11 \\
0.15\end{array}$ & $\begin{array}{l}1.11 \\
1.11\end{array}$ & $\begin{array}{l}1: 11 \\
1.11\end{array}$ & $\begin{array}{l}0.14 \\
0.15\end{array}$ & $\begin{array}{l}0.01 \\
0.05\end{array}$ & $\begin{array}{l}\text { e.11 } \\
0.15\end{array}$ & $\begin{array}{l}1.11 \\
0.11\end{array}$ & $\begin{array}{l}0.01 \\
0.01\end{array}$ & $\begin{array}{l}0.11 \\
0.01\end{array}$ \\
\hline 1.02 & 1.12 & 1.02 & 0.12 & 1.12 & 1.0? & 0.02 & 1.12 & 1.02 & 1.12 & 1.02 & 1.01 & 0.12 & 0.01 & 0.02 & 0.62 & 1.01 & 1.02 \\
\hline $\begin{array}{l}11.19 \\
11.11\end{array}$ & $\begin{array}{l}11.128 \\
11.15\end{array}$ & $\begin{array}{r}2.15 \\
10.15\end{array}$ & $\begin{array}{l}1.61 \\
1.31\end{array}$ & $\begin{array}{l}1.61 \\
1.31\end{array}$ & $\begin{array}{l}1.01 \\
1.75\end{array}$ & $\begin{array}{l}6.21 \\
1.01\end{array}$ & $\begin{array}{l}\text { S.15 } \\
\text { (.113 }\end{array}$ & $\begin{array}{l}1.61 \\
1.26\end{array}$ & $\begin{array}{l}1.112 \\
1.61\end{array}$ & $\begin{array}{l}6.01 \\
6.81\end{array}$ & $\begin{array}{l}\text { 6.19 } \\
\text { (.193 }\end{array}$ & $\begin{array}{l}6.11 \\
6.02\end{array}$ & $\begin{array}{l}5.69 \\
6.11\end{array}$ & $\begin{array}{l}5.18 \\
3.62\end{array}$ & $\begin{array}{l}6.11 \\
\text { s.11 }\end{array}$ & $\begin{array}{l}1.71 \\
5.11\end{array}$ & $\begin{array}{l}1.10 \\
1.55\end{array}$ \\
\hline
\end{tabular}


scrimio is coir.

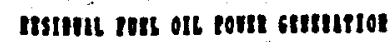

IIII

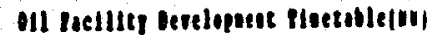
Potil tet copetif tevelopinte

OII irice (I III)

Ilectrlelty irodeed liomary)

oil enined (I thi)

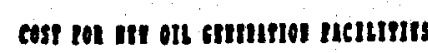

eipital

otit

Intoietrative Inpuese (II)

cout (If)

IIth Profit (II)

cotertin (oll)

ciplel
oir
inel

Inidetritive enpenis

Inel tentairm

fital ceutu/rn ifll profte

$\boldsymbol{n}$ u $\boldsymbol{u}$

$26 \quad 21$

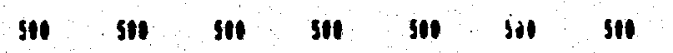

$15.12 \quad 15.01$ is.12 15.02 is.12 15.02 is.12 $15.11 \quad 18.12$

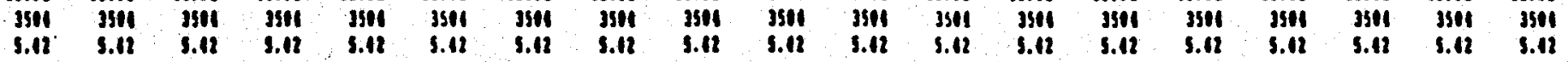

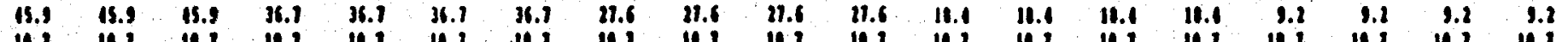

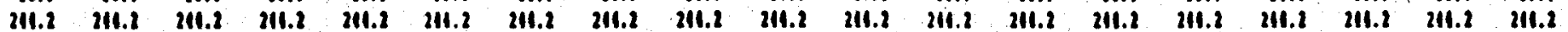

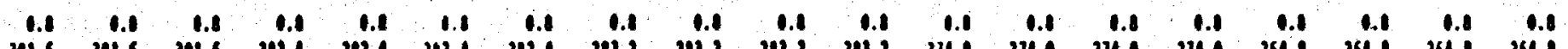

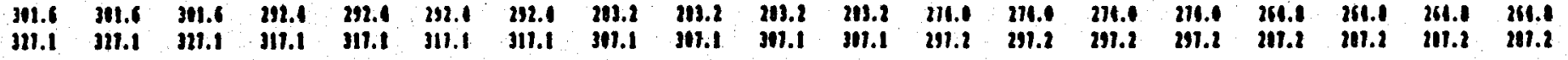

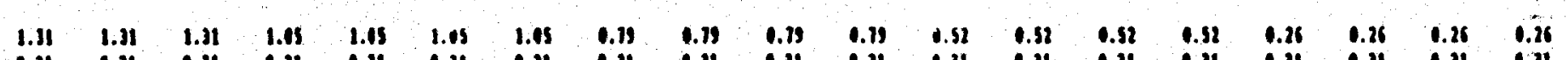

$\begin{array}{llllllllllllllllllll}.31 & 0.31 & 0.31 & 0.31 & 0.31 & 0.31 & 0.31 & 0.31 & 0.31 & 0.31 & 0.31 & 0.31 & 0.31 & 0.31 & 0.31 & 0.31 & 0.31 & 0.31 & 0.31\end{array}$

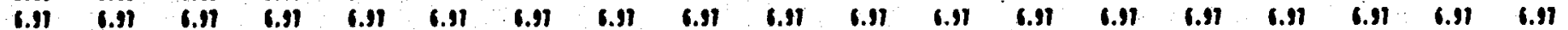

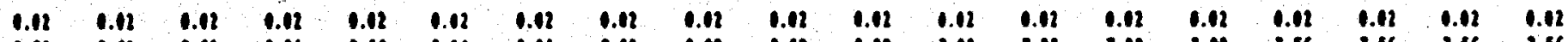

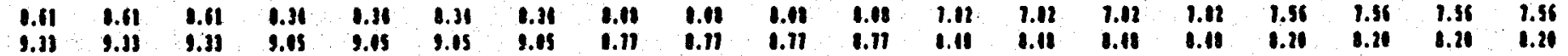

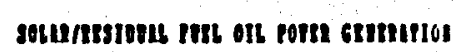

me

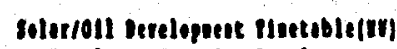

tetel let copetty terelepeit

Ilectrletty Irolecel (Inomm)

solut Psoheet tlectrielty |lowsur|

ofl Etles (I III)

oll sobetl tleetrielty (10oumb)

oll cosmonet (I Bil)

cosp ror solutort efmention nembirts Copital.

Inel

Ihioletrative Brpenes (un)

Cost (III)

Ittl Proflt (II)

consolem

enpieal

oin

Inlatertutine enperes

inta! teotertn

Plal entuirm itt protst

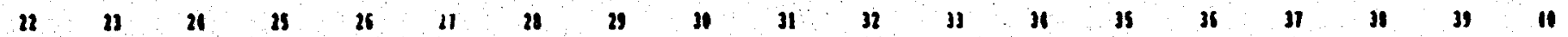

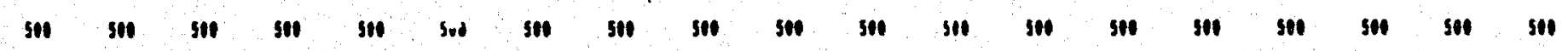

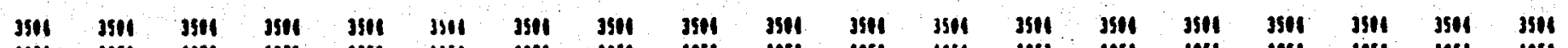

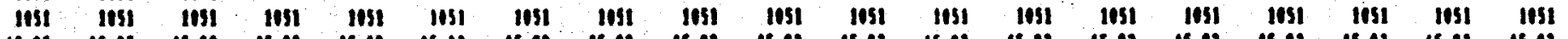

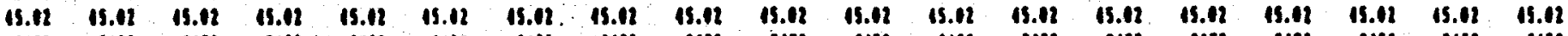

$21512153 \quad 2153 \quad 2153 \quad 2153 \quad 2153 \quad 2151 \quad 2153 \quad 2153 \quad 2153 \quad 2153 \quad 2153 \quad 2153 \quad 2153 \quad 2153 \quad 2153 \quad 2153 \quad 2153 \quad 2153$

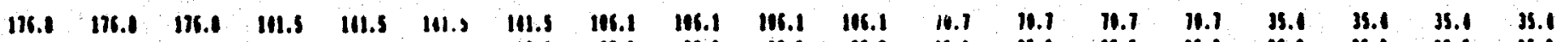

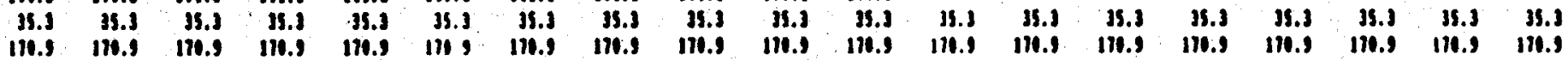

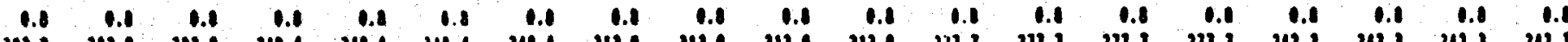

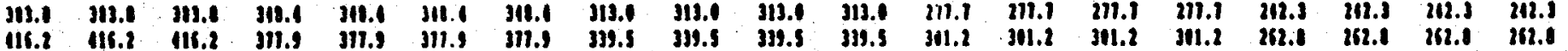

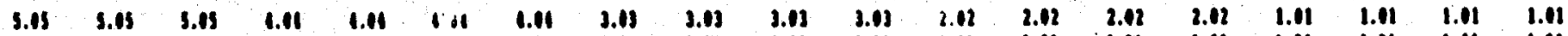

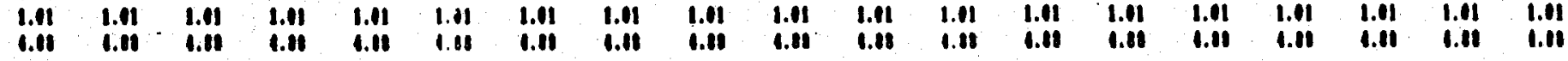

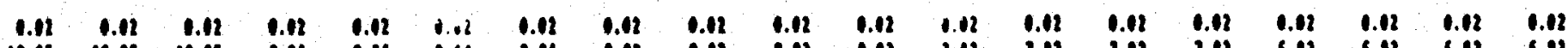

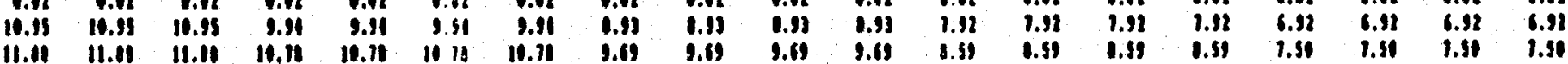




\section{SCENARIO 4}

$50 \mathrm{MW}$ power plants using high plant/wellfield costs and a $20 \%$ contingency 


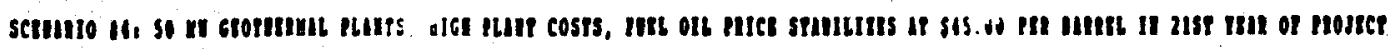
ceormenil corme stmutros III:

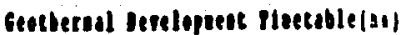
potal tet capactet berelopued

Electriclty Prodicel (1010um)

cons ron esomment corn nossef Geothernal Rlants (If)

eniten

Replaceseat vells

ost

Poylte tupeats

Ient

Jint teplocent lon

colles ind toetlltes (II)

cuitul

ofi

Malofotratire Imeuses (n)

cont (iv)

iles protle (I)

centa/ru (sentierwal)

Seotlernel Blute

Cipltal

Beplocenut vell.

ofr

Eprelty Bopento

Plint Inplocenet loes

cilles in Paeflities

enital

oft

Inlolatratine Inposes

Cente/rn

centertu (ulth grotit) 
senese 11 cons.

thimb tre oft rom semtrios

IIII

Oll Retlity Direlopunt Ploctutle idt

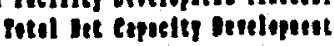

oll telee |f |tII)

Iletelelty Proleced (Ionim)

oll tonsmel (t) itI)

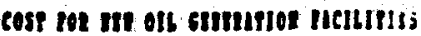
entent

oin

inil

$9.29 .1,9.2^{12}$

Intoletrative inpesues ins cone (InI)

IIt Profit (ms)

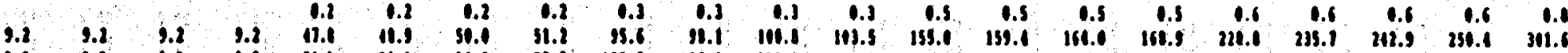

ceiten (uil)

ontin:

inel

Inlsionetin tmpenes

Totol cetterm

fotel Ceste/m rith profle

1.3 1.31 l. 13

$$
10
$$

$11 \quad 12$

13 is 11 11 $11 \quad 11 \quad 18 \quad 21$

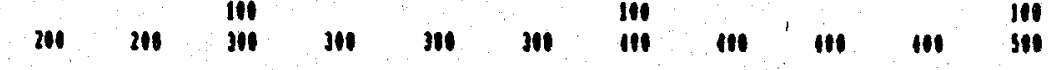

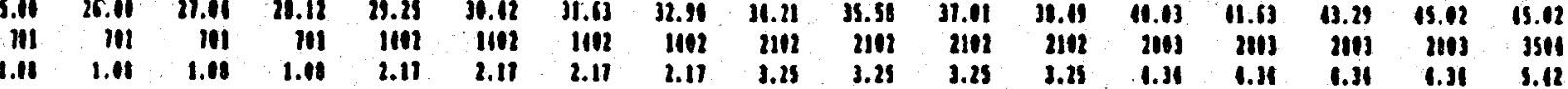

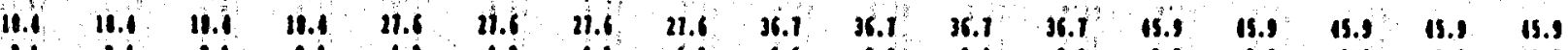

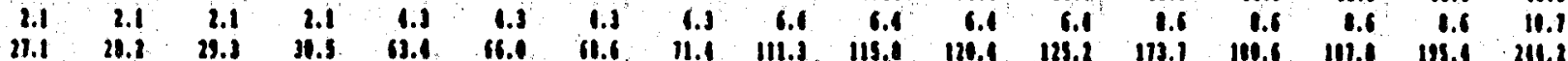

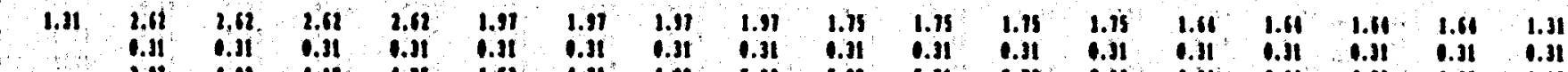

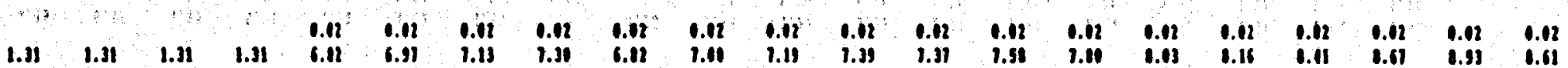

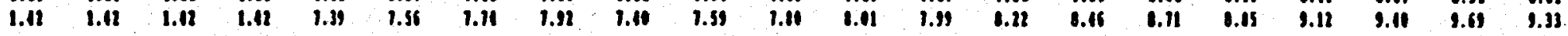

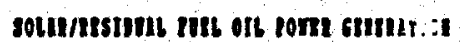
rin

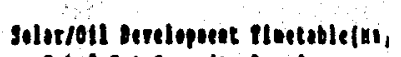
Potal int Capetsy Devilopest

Sleserleits frolienl (Iontry)

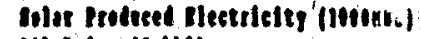

oll telee |f bil|

oll Probeil bietriedty liomuri

OII Cossened (I bil)

cost ion soluent grimesor nethits cepteal

oft.

andotuttatle sipenes [as]

Coit (if)

III Profte (II)

cintoirm

capitul

Inel

Mololotratine Ispenes

Petul cistion

fotal centurm ulto protes 
seminio 11 cost.

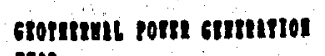

mi

Ceotieral oevelopent phetable(:d)

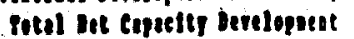

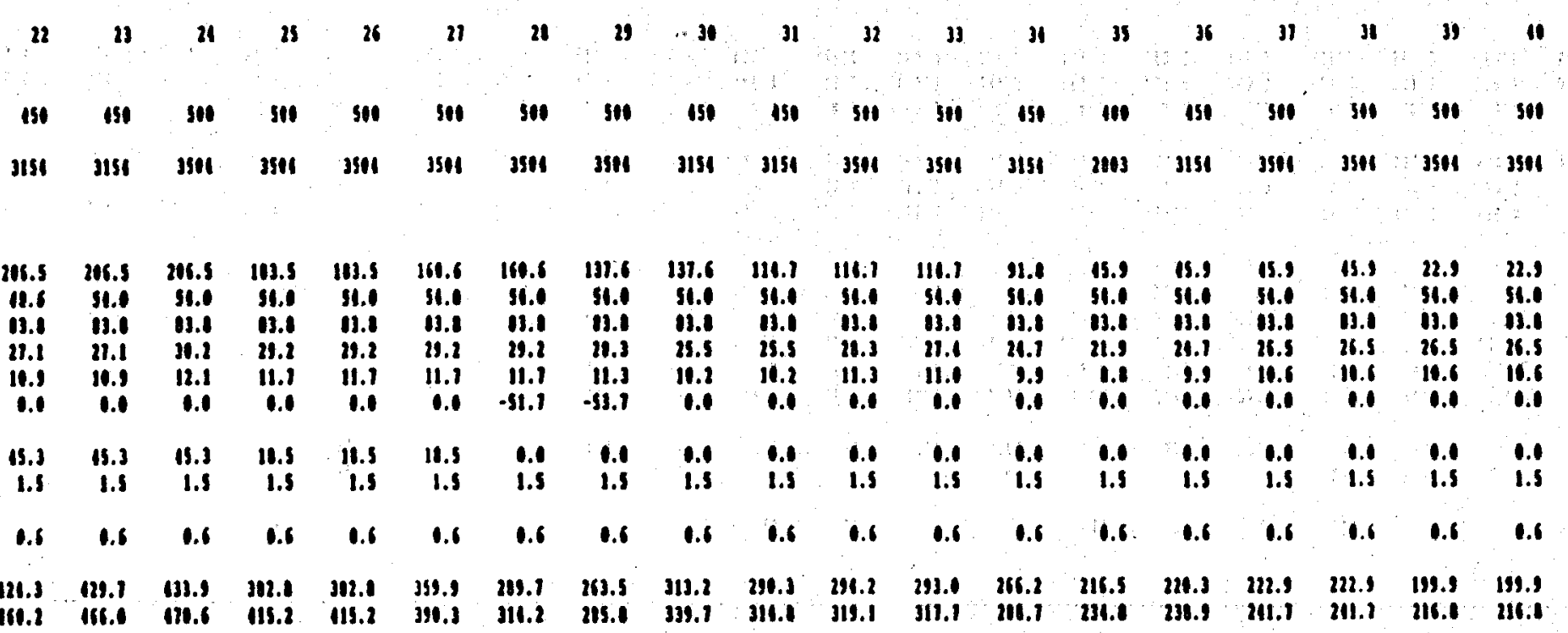

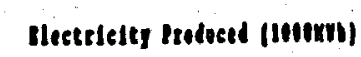

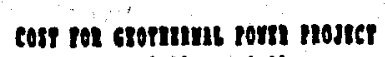
ceodenul Put! (If)

$$
\text { Capieal }
$$

ceplocente inls

ofi

Ioyalty Pupest:

Inet

Plate Replecentit rout

Cables oud Heflitle! (II)

Capltal

of

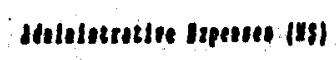

cont InII

IIII protlt (In)

cetteirm (geotletwi)

ceotlernal pluts.

captul

Iepleseret Hills

ois

loputty tepoents

Iins

llast Ieplacenent Ind

colles an feellities

copltal

ofil

Inidintratire Ispenes

Centirtm

Cestolir (vith profte)

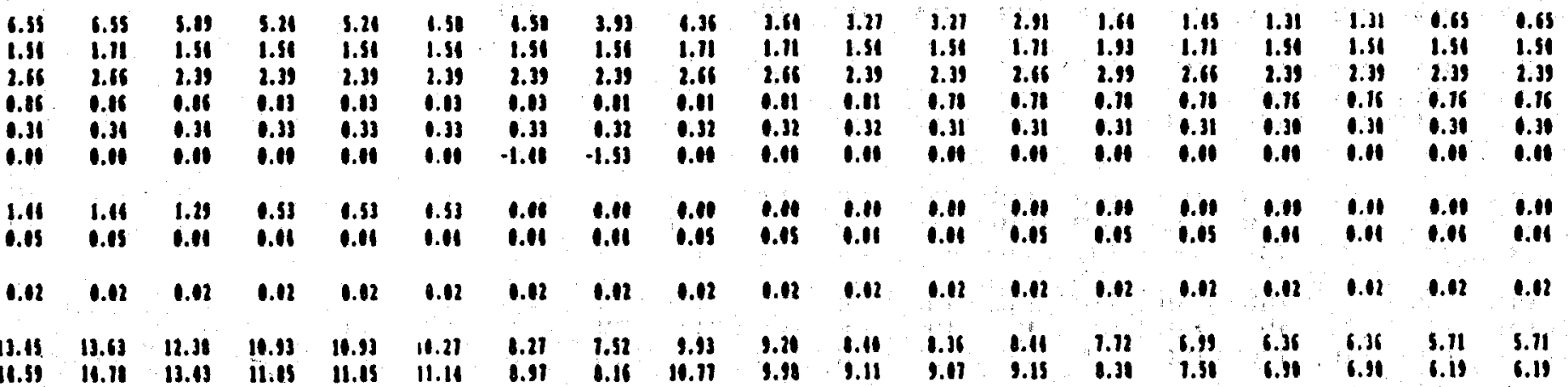


scmmo 11 cons.

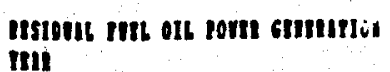

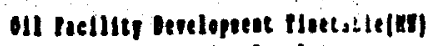

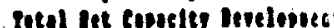

oll Irice (f $\mathrm{ml})$

Dleetrieteg troluced (100mm)

oll conenet (I) bill

cost rot un ort smmitios nemitits

cipite!

Iof!

Iniolotratle Ingenes (afl

cost (in)

Itis Priflt (II)

22 23 2 25 20 n 11

s11 311 sit s11

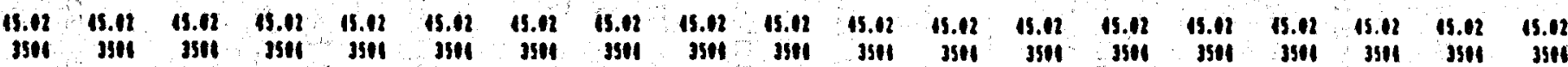

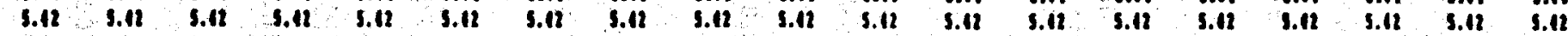

centu/In (oll)

taptis:

ofi.

Inet

Inidetrative Impense

Porel cintertit

Potel cettorn olst pioft

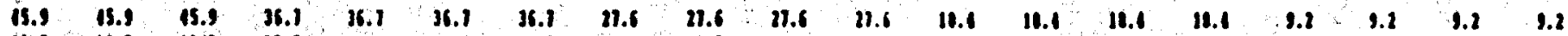

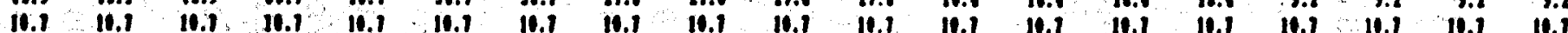

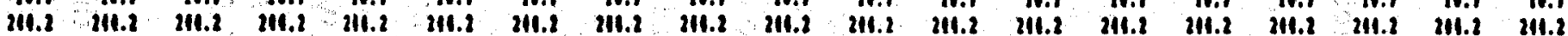

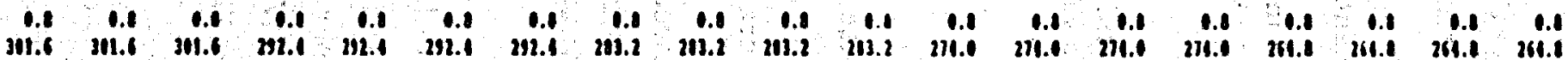

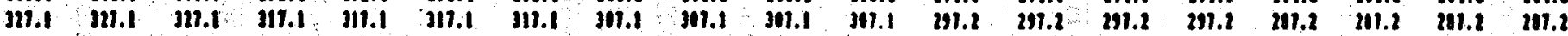

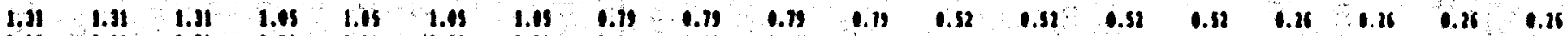

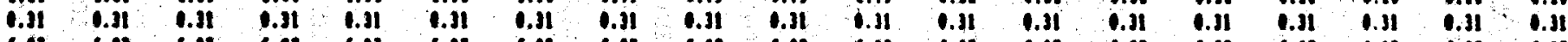

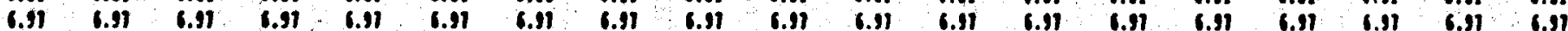

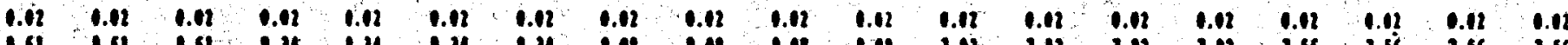

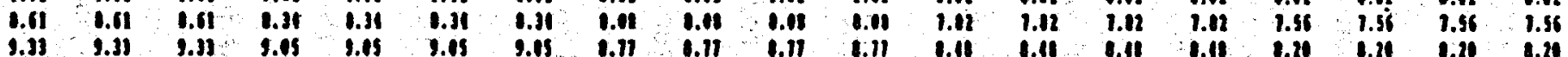

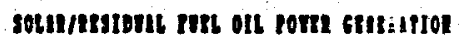

Im

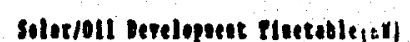
Potil let Capaclty berelepest

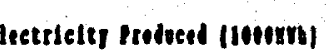

silet Irodieed Bleetrielty (llici..ut)

oll Pelee If bil|

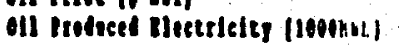

oll Cossuril (I bll)

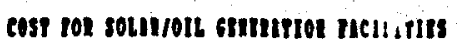
copitei

ofl

Ihiolientles Itpenece (z)

cont (tis)

IIt iroflt (II)

cestorm

eipitsl

int

Itofolotrotive tipenes

entil contisn

Potul cisturtt otel protit

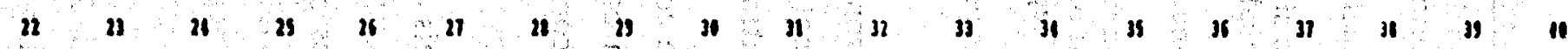

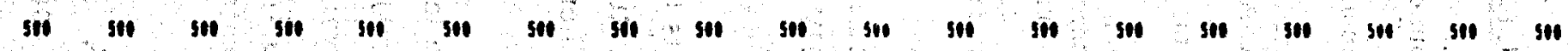

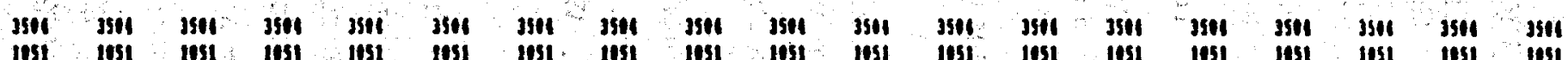

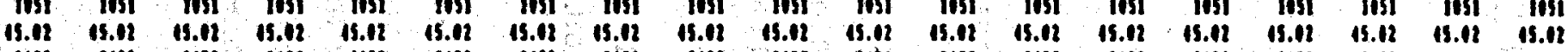

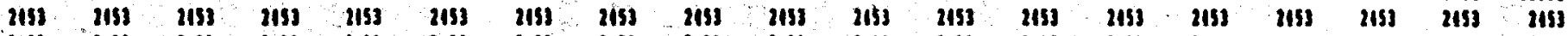

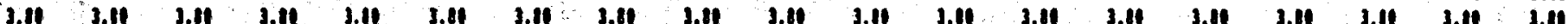

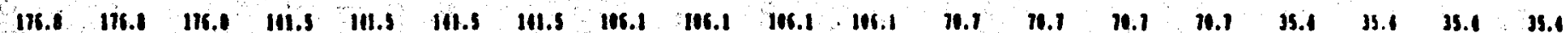

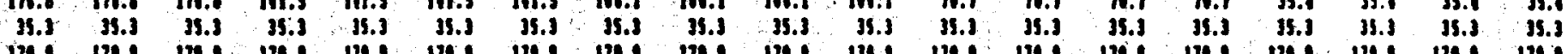

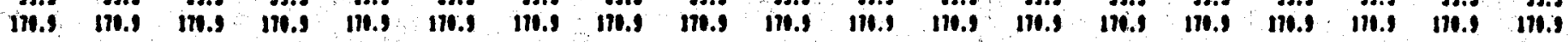

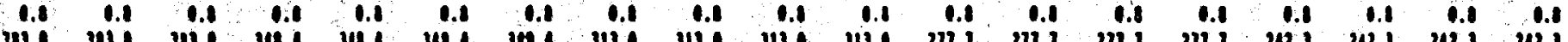

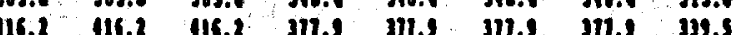

\begin{tabular}{|c|c|c|c|c|c|c|c|c|c|c|c|c|c|c|c|c|c|}
\hline $\begin{array}{l}\text { s.15 } \\
1.01 \\
1.01\end{array}$ & $\begin{array}{l}5.115 \\
1.01 \\
1.81\end{array}$ & $\begin{array}{l}3.113 \\
1.11 \\
1.11\end{array}$ & $\begin{array}{l}1.11 \\
1.11 \\
1.18\end{array}$ & $\begin{array}{l}1.11 \\
1.11 \\
1.11\end{array}$ & $\begin{array}{l}1.01 \\
1.11 \\
1.11\end{array}$ & $\begin{array}{l}1.01 \\
1.11 \\
1.01\end{array}$ & $\begin{array}{l}3.03 \\
1.11 \\
1.01\end{array}$ & $\begin{array}{l}3.01 \\
1.01 \\
1.01\end{array}$ & $\begin{array}{l}3.01 \\
1.11 \\
1.11\end{array}$ & $\begin{array}{l}3.11 \\
1.11 \\
1.11\end{array}$ & $\begin{array}{l}2.12 \\
1.11 \\
1.11\end{array}$ & $\begin{array}{l}2.02 \\
1.01 \\
1.01\end{array}$ & $\begin{array}{l}2.12 \\
1.11 \\
1.10\end{array}$ & $\begin{array}{l}2.11 \\
1.11 \\
1.11\end{array}$ & $\begin{array}{l}1.11 \\
1.11 \\
1.81\end{array}$ & $\begin{array}{l}1.11 \\
1.11 \\
1.11\end{array}$ & $\begin{array}{l}1.01 \\
1.01 \\
1.11\end{array}$ \\
\hline $\begin{array}{l}0.1 \\
0.1\end{array}$ & $\begin{array}{r}1.11 \\
11.91\end{array}$ & $\begin{array}{c}1.11 \\
11.15\end{array}$ & $\begin{array}{l}1.02 \\
1.91\end{array}$ & $\begin{array}{l}1.01 \\
9.91\end{array}$ & $\begin{array}{l}1.11 \\
.11\end{array}$ & $\begin{array}{l}1.12 \\
9.11\end{array}$ & $\begin{array}{l}1.11 \\
1.13\end{array}$ & $\begin{array}{l}1.12 \\
8.83\end{array}$ & $\begin{array}{l}1.01 \\
1.91\end{array}$ & $\begin{array}{l}1.12 \\
1.13\end{array}$ & $\begin{array}{l}1.02 \\
1.92\end{array}$ & $\begin{array}{l}1.12 \\
1.12\end{array}$ & $\begin{array}{l}1.11 \\
1.12\end{array}$ & $\begin{array}{l}1.02 \\
1.22\end{array}$ & & & $\begin{array}{l}1.12 \\
6.12\end{array}$ \\
\hline 1.8 & 11.18 & il.eit & $11 . n$ & $11 . n$ & $11 . n$ & 10.11 & 1.69 & is: & 39 & 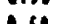 & 8 & 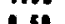 & $\because 3$ & 3 & 158 & 1.50 & 1.51 \\
\hline
\end{tabular}




\section{SOLAR/RESIDUAL FUEL OIL POWER GENERATION cont'd}

O\&M

Fuel

Administrative

Expenses

Cost

With Profit
The annual O\&M costs of the power plants. (Northwest Power Planning Council estimate.)

The annual fuel costs of the power plants (oil consumed times oil price).

Based on similar costs per $\mathrm{kWh}$ found in Hawaiian Electric Industries, Inc. Annual Report.

Total annual project cost.

Total annual project cost with $8 \%$ profit. Profit is calculated as a percent of annual costs based on Hawaiian Electric Industries, Inc, Annual Report.

The second set of costs is in cents per kilowatt hour.

Tables $16,17,18$, and 19 compare the costs of the three generation alternatives under $25 \mathrm{MW}$ and $50 \mathrm{MW}$ geothermal options with low and high plant/wellfield cost estimates using a $20 \%$ contingency for all three generation alternatives.

With $25 \mathrm{MW}$ plants the cost of geothermal is between 19 and 23 times more costly than the oil generation option. With $50 \mathrm{MW}$ plants, generation is 17 to 20 times as costly as oil. When compared to solar/oil, geothermal is 12 to 17 times as costiy.

Table 20 shows the levels of rate increases that would have to be achieved to cover the cost of adding $500 \mathrm{MW}$ of power generating capacity to the system over a 40 year period. With royalties paid to the state, rates per $\mathrm{kWh}$ could be expected to increase on average by $17 \%$ ( $50 \mathrm{MW}$ low) to $30 \%$ (25 MW high) if the geothermal option is taken while a rate increase of $10 \%$ for oil and $17 \%$ for solar/oil could be expected.

Tables 21, 22, 23, and 24 compare the costs of the three generation alternatives using a $30 \%$ contingency for geothermal and $20 \%$ for oil and solar/oil. Table 25 shows the levels of rate increases with the $30 \%$ contingency level. 
Table 16

\section{NET MW SUMMARY TABLE \\ LOW GEOTHERMAL COSTS 20\% CONTINGENCY}

\begin{tabular}{lccc}
\hline & Geothermal & Solar/Oil & Oil \\
\hline \hline (1,000 MWh Produced) & 101,791 & $\begin{array}{c}98.112 \\
\end{array}$ & 98,112 \\
& & $30 \%$ solar & \\
Total Oil Consumed $(\mathrm{M} \mathrm{bbl)}$ & 0 & $10 \%$ oil & \\
BS Development Cost & 3.6 & 21 & 152 \\
BS 40 Yr. Cumulative Cost & 121 & 10.6 & 0.5 \\
BS NPV & 4.9 & 3.6 & 8.6 \\
Cost Ratio (oil =1.0) & 1.55 & 1.37 & 27 \\
Cost Ratio (solar/oil $=10)$ & 136 & 1.00 & 1.00 \\
Levelized Cents/kWh & 1187 & 10.81 & 0.73 \\
\hline \hline
\end{tabular}

Table 17

25 NET MW SUMMARY TABLE

HIGH GEOTHERMAL COSTS $20 \%$ CONTINGENCY

\begin{tabular}{lccc}
\hline & Geothermai & Solarioil & Oil \\
\hline (1,000 MWh Produced) & 101,791 & $\begin{array}{l}98,112 \\
\end{array}$ & 98,112 \\
& & $30 \%$ solar & \\
Total Oil Consumed (M bbl) & 0 & $70 \%$ oil & \\
BS Development Cost & 4.3 & 106 & 152 \\
BS 40 Yr. Cumulative Cost & 15.0 & 21 & 05 \\
BS NPV & 6.0 & 10.6 & 86 \\
Cost Ratio (oil $=10)$ & 225 & 3.6 & 27 \\
Cost Ratio (solar/oil $=1.0)$ & 165 & 137 & 100 \\
Levelized Cents/kWh & 14.71 & 100 & 0.73 \\
\hline \hline
\end{tabular}


Table 18

\section{NET MW SUMMARY TABLE \\ LOW GEOTHERMAL COSTS 20\% CONTINGENCY}

\begin{tabular}{lcrc}
\hline & Geothermal & Solar/Oil & Oil \\
\hline \hline (1,000 MWh Produced) & 98,813 & 98,112 & 98,112 \\
& & $30 \%$ solar & \\
Total Oil Consumed (M bbl) & 0 & $70 \%$ oil & 152 \\
BS Development Cost & 3.4 & 106 & 0.5 \\
BS 40 Yr. Cumulative Cost & 108 & 21 & 8.6 \\
BS NPV & 45 & 10.6 & 2.7 \\
Cost Ratio (oil = 10) & 168 & 3.6 & 100 \\
Cost Ratio (solar/oil = 10) & 123 & 1.37 & 0.73 \\
Levelized Cents/kWh & 10.97 & 1.00 & 873 \\
\hline \hline
\end{tabular}

Table 19

50 NET MW SUMMARY TABLE

HIGH GEOTHERMAL COSTS 20\% CONTINGENCY

\begin{tabular}{lcrc}
\hline & Geothermal & Solar Oil & Dil \\
\hline \hline (1,000 MWh Produced) & 98,813 & 98,112 & 98,112 \\
& & $30 \%$ solar & \\
Total Oil Consumed (M bbl) & 0 & $70 \%$ oil & \\
BS Development Cost & 4.0 & 106 & 152 \\
BS 40 Yr. Cumulative Cost & 13.4 & 21 & 0.5 \\
BS NPV & 5.4 & 10.6 & 8.6 \\
Cost Ratio (oil = 10) & 2.03 & 3.6 & 27 \\
Cost Ratio (solar/oil =10) & 149 & 137 & 100 \\
Levelized Cents/kWh & 1359 & 100 & 0.73 \\
\hline \hline
\end{tabular}


IABLE 21

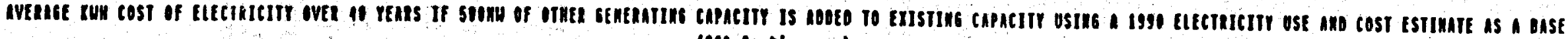
(21) Contingeney)

(2/1 Hell Reploeenent)

Existing ststei (199))

$11,151,281,111$ Tetal inh Cipuelty $1,261,319,321$ uith 231 Reservo

(13,911,as2 \& operating Revenue $1,261,319,321$ innuel thit sold

1.11 Average contr/hus

Georatenn

104

nODE CAPACITY

(25nu Plants)

(11) rearis)

$12,011,111,010$ Project tost

$\infty 111,191,011,010$

ith sold

11.81 iverage conts/tith with Roralty

coneine chpectry

(11) reors)

$36,638,994,011$ operat lng Revenes $118,117,015,315$ the sold

8.96 Iverage conts/tuh

Cents/kun Incresse 1.53 With iddes capreity.

Cente/twh I Increase 20.6t WIth idded Capicliy

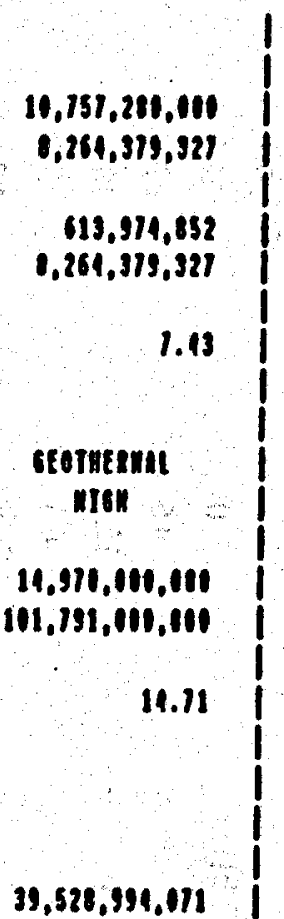

412, $111,045,306$
ExIsITU STSTE

(iss)

$11,757,201,010$ Total the cepaclty

$0,261,319,327$ Uith 231 leserve

$613,911,852$ oporatlag lovenye

$8,264,315,321$ innoul tuh soll

1.13 iverago cents/huh

ofothernnt

100

coneo capheITY

(soin plants)

(1) Teire)

11,011, int,ill 1 Project cost

$98,813,311,111$ kith sold

10.91 Iverage cents/hith iteh repolty

coneruto chpesity

(11 resre)

$39,396,991,011$ \& operating Revenua $31,988,991,011$

116,169,169,419 the sold

8.11 Iverage tente/kuth

Conts/kut Increase

1.21 With idded capicity

Conte/kuh I Incroses

11.28 Uth inded capacity

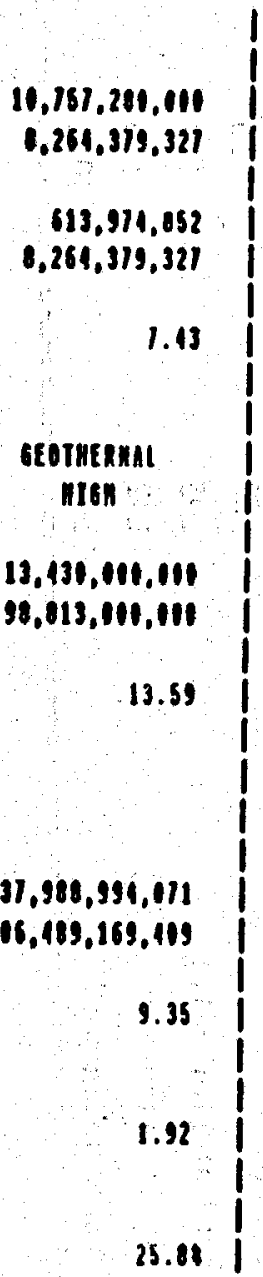

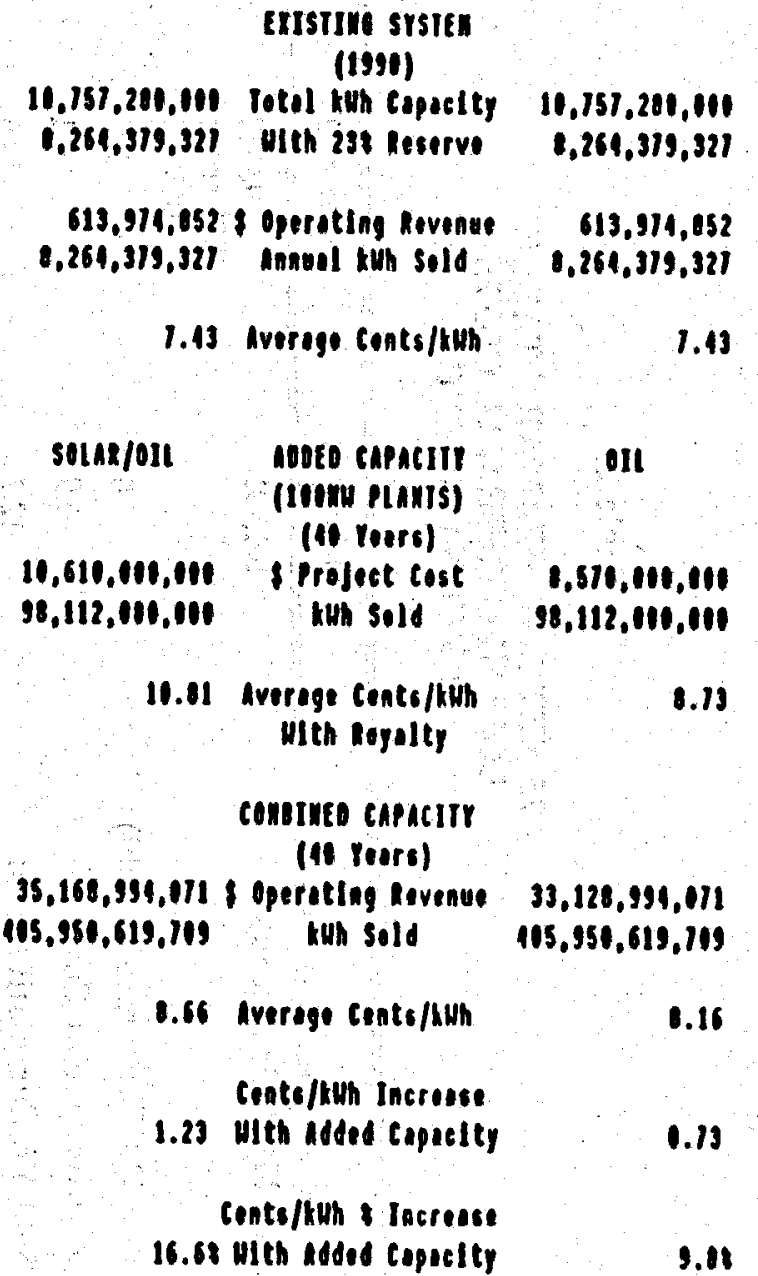

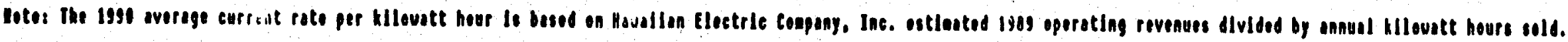




\section{Table 21}

25 NET MW SUMMARY TABLE

LOW GEOTHERMAL COSTS 30\% CONTINGENCY

\begin{tabular}{lccc}
\hline & Geothermal & Solar/Oil & Oil \\
\hline \hline (1,000 MWh Produced) & 101,791 & 98,112 & 98,112 \\
& & $30 \%$ solar & \\
Total Oil Consumed (M bbl) & 0 & $70 \%$ oil & 152 \\
BS Development Cost & 3.9 & 106 & 0.5 \\
BS 40 Yr. Cumulative Cost & 12.7 & 21 & 86 \\
BS NPV & 5.2 & 10.6 & 27 \\
Cost Ratio (oil =10) & 1.95 & 3.6 & 100 \\
Cost Ratio (solar/oil $=10)$ & 1.42 & 137 & 0.73 \\
Levelized Cents/kWh & 12.46 & 1.00 & 873 \\
\hline \hline
\end{tabular}

Table 22

25 NET MW SUMMARY TABLE HIGH GEOTHERMAL COSTS $30 \%$ CONTINGENCY

\begin{tabular}{|c|c|c|c|}
\hline & Geothermal & Solarivil & Oil \\
\hline (1,000 MWh Produced) & 101,791 & $\begin{array}{r}98,112 \\
30 \% \text { solar } \\
70 \% \text { oil }\end{array}$ & 98,112 \\
\hline Total Oil Consumed (M bbl) & 0 & 106 & 152 \\
\hline BS Development Cost & 4.6 & 21 & 0.5 \\
\hline BS 40 Yr. Cumulative Cost & 15.7 & 10.6 & 86 \\
\hline B $\$$ NPV & 6.3 & 3.6 & 27 \\
\hline Cost Ratio (oil $=10$ ) & 236 & 137 & 100 \\
\hline Cost Ratio (solar/oil $=10$ ) & 1.73 & 100 & 0.73 \\
\hline L.evelized Cents/kWh & 15.43 & 19.81 & $\$ 73$ \\
\hline
\end{tabular}


Table 23

50 NET MW SUMMARY TABLE

LOW GEOTHERMAL COSTS

30\% CONTINGENCY

\begin{tabular}{lccc}
\hline \hline & Geothermal & Solar/Oil & Oil \\
\hline \hline$(1,000$ MWh Produced) & 98,813 & $\begin{array}{c}98,112 \\
\end{array}$ & 98,112 \\
& & $30 \%$ solar & \\
Total Oil Consumed $(\mathrm{M} \mathrm{bbl})$ & 0 & $10 \%$ oil & \\
BS Development Cost & 3.6 & 106 & 152 \\
BS 40 Yr. Cumulative Cost & 114 & 21 & 0.5 \\
BS NPV & 4.7 & 10.6 & 8.6 \\
Cost Ratio (oil = 1.0) & 1.76 & 3.6 & 27 \\
Cost Ratio (solar/oil $=10)$ & 129 & 137 & 1.00 \\
Levelized Cents/kWh & 1149 & 100 & 0.73 \\
\hline \hline
\end{tabular}

Table 24

SO NET MW SUMMARY TABLE HIGH GEOTHERMAL COSTS $30 \%$ CONTINGENCY

\begin{tabular}{lccc}
\hline & Geothermal & Solar/Oil & Oil \\
\hline \hline (1,000 MWh Produced) & 98,813 & 98,112 & 98,112 \\
& & $30 \%$ solar & \\
Total Oil Consumed (M bbl) & 0 & $70 \%$ oil & 152 \\
BS Development Cost & 43 & 106 & 05 \\
BS 40 Yr. Cumulative Cost & 14.1 & 21 & 86 \\
BS NPV & 5.7 & 10.6 & 27 \\
Cost Ratio (oil = L0) & 213 & 3.6 & 100 \\
Cost Ratio (solar/oil $=10)$ & 1.56 & 137 & 0.73 \\
Livvelized CintsikWh & 14.24 & 100 & $\$, 3$ \\
\hline \hline
\end{tabular}


IABLE 25

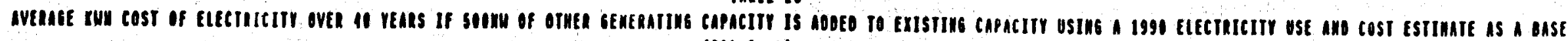
(301 Contingency)

(2/1 Noll Replaceennt)

\begin{tabular}{|c|c|c|c|c|c|c|c|c|}
\hline & $\begin{array}{c}\text { ExISIIUG SYSIEN } \\
\text { (1951) }\end{array}$ & & & $\begin{array}{l}\text { EIIsIIME systen } \\
\text { (I9sI) }\end{array}$ & & & $\begin{array}{l}\text { ExISIIUG SYSYE" } \\
\text { (I990) }\end{array}$ & \\
\hline $\begin{array}{l}11,131,261,011 \\
1,261,319,321\end{array}$ & $\begin{array}{l}\text { Total tuh capocity } \\
\text { UIth 23t leserve }\end{array}$ & $\begin{array}{r}11,161,211,011 \\
1,264,379,321\end{array}$ & $\begin{array}{r}11,751,281,111 \\
1,264,319,321\end{array}$ & $\begin{array}{l}\text { Total tuh cipielty } \\
\text { ulth 23t Recerve }\end{array}$ & $\begin{array}{r}11,151,201,011 \\
8,261,319,321\end{array}$ & $\begin{array}{r}11,751,281,011 \\
8,261,319,321\end{array}$ & $\begin{array}{l}\text { Total twh Copacity } \\
\text { UIth } 239 \text { Reserve }\end{array}$ & $\begin{array}{r}11,151,281,011 \\
1,261,319,321\end{array}$ \\
\hline $\begin{array}{r}113,911,052 \\
1,261,319,321\end{array}$ & $\begin{array}{l}\text { I Operetlng Revesue } \\
\text { enneal kHh sold }\end{array}$ & $\begin{array}{r}613,911,062 \\
0,261,379,321\end{array}$ & $\begin{array}{r}613,911,152 \\
1,261,319,321\end{array}$ & $\begin{array}{l}\text { I Operoting Revonus } \\
\text { enneol kuh sold }\end{array}$ & $\begin{array}{r}613,914,852 \\
0,261,379,321\end{array}$ & $\begin{array}{r}613,911,452 \\
8,261,379,321\end{array}$ & $\begin{array}{l}\text { Oporating lovonue } \\
\text { ennual thith sold }\end{array}$ & $\begin{array}{r}613,911,152 \\
0,261,319,321\end{array}$ \\
\hline 1.13 & average $t_{t}\left(1, t_{s} / \mathrm{k}\right.$ th & 1.13 & 1.13 & Ivernge Cealo/kuh & 1.13 & 1.13 & Averege Cents/kuth & 1.13 \\
\hline $\begin{array}{l}\text { Geotuenulat } \\
\text { LOU }\end{array}$ & $\begin{array}{l}\text { AODEO CApActir } \\
\text { (2snu planrs) } \\
\text { (11 Tears) }\end{array}$ & $\begin{array}{l}\text { GEOTuERul } \\
\text { nIEn }\end{array}$ & $\begin{array}{l}\text { AEOThEAMA } \\
\text { LOH }\end{array}$ & 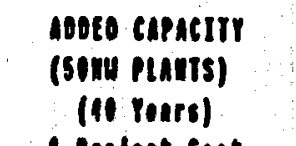 & $\begin{array}{l}\text { oformernal } \\
\text { nifn }\end{array}$ & solin/oIt & 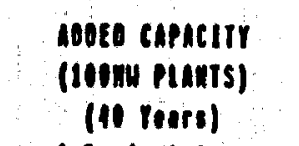 & OII \\
\hline $111,791,011,111$ & $\begin{array}{l}\text { Piroject cost } \\
\text { the sold }\end{array}$ & $\begin{array}{l}19,110,011,011 \\
111,191,011,111\end{array}$ & $\begin{array}{l}11,351,111,011 \\
91,813,111,011\end{array}$ & $\begin{array}{l}1 \text { Project cost } \\
\text { tub sold }\end{array}$ & $\begin{array}{l}11,011,011,111 \\
98,813,111,111\end{array}$ & $\begin{array}{l}11,611,011,111 \\
91,112,011,111\end{array}$ & $\begin{array}{l}\text { Projeet coit } \\
\text { twh sold }\end{array}$ & $\begin{array}{r}1,311,111,111 \\
98,112,111,111\end{array}$ \\
\hline 12.16 & $\begin{array}{l}\text { Avorage Cecits/tuh } \\
\text { usth Royalty }\end{array}$ & 15.13 & 11.49 & $\begin{array}{l}\text { Average cents/kuth } \\
\text { With leyelty }\end{array}$ & 11.21 & 11.81 & $\begin{array}{c}\text { Averoge Conto/kuh } \\
\text { uith Ropalty }\end{array}$ & 8.13 \\
\hline $31,238,994,671$ & $\begin{array}{l}\text { Conbined CovacITY } \\
\text { (lit Veors) } \\
\text { I operoting Rovenue }\end{array}$ & $1,261,994,011$ & $35,918,941,011$ & $\begin{array}{l}\text { Colloined capacily } \\
\text { (11 reore) } \\
\text { f oporetleg Rovenue }\end{array}$ & 191,011 & & 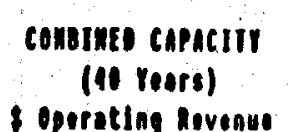 & \\
\hline $11.111,115,316$ & & $111,111,115,301$ & $116,119,169,119$ & kuh sold & $106,169,169,419$ & 105,951, 619,1199 & kWh sold & $\begin{array}{r}115,951,619,119 \\
\text {. }\end{array}$ \\
\hline 9.11 & Average Cencs/kuh & 9.85 & 8.83 & Average Conts/keh & 9.51 & 8.66 & Avorage Conte/suh & 0.16 \\
\hline 1.68 & $\begin{array}{l}\text { Conts/luh licresse } \\
\text { ulth noded capeclty }\end{array}$ & 2.12 & 1.11 & $\begin{array}{l}\text { Ceats/kuh Ineresee } \\
\text { WIth udded capecity }\end{array}$ & 2.11 & 1.23 & $\begin{array}{l}\text { Conte/kuh Ineresse } \\
\text { ulth noded copocity }\end{array}$ & 0.13 \\
\hline 22.68 & seed c & 32.68 & 18.98 & $\begin{array}{l}\text { Cente/kth I Inerease } \\
\text { ulth ndsed Copectey }\end{array}$ & 21.98 & 16.68 & $\begin{array}{l}\text { Ceats/kuh I locrosie } \\
\text { uIth nded copocity }\end{array}$ & 9.11 \\
\hline
\end{tabular}

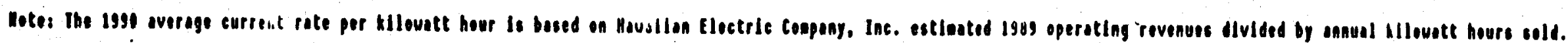




\section{NEA - CONSERVATION AND SOLAR ENERGY}

Conservation can be a key resource for meeting Hawaii's future electrical energy needs. Each megawatt of electricity conserved is one less megawatt that needs to be generated Utilities all across the country are beginning to see conservation as a resource much less costly than the addition of new generating facilities. An article on energy appearing in The Nation's Business, February 1990 states:

Experts say that universal adoption of standard conservation measures, such as insulation, recycling, and other practices. could cut U.S. energy consumption by as much as 20 percent. Even more energy could be saved, they add through broader application in business and industry of new technologies such as high-efficiency lighting, automatic controls, heat pumps, adjustable-speed drives, and thermal storage.

A major boost for conservation is coming from utilities, which see programs to reduce demand as attractive alternatives to building more plants or buying electricity from independent producers. Financial incentives are available in many areas to business and individual users who agree to install energysaving equipment or to cut back voluntarily on energy consumption.

Conservation no longer means "freezing in the dark" or "lowering your standard of living" as critics like to contend. It means being smart it means ceing efficient, and that means it makes good business sense. The Northwest Power Planning Council, the organization in the Pacific Northwest responsible for energy planning defines conservation in the following way.

Conservation refers to the more efficient use of electricity-not curtailmentthat results in the reduction of consumption. This means that less electricity is used to support the same level of amenity or production that existed before the conservation measure was implemented. Conservation resources are measures that enable residential and commercial buildings, appliances, and industrial and irrigation processes to use energy efficiently.

1/ 1989 Supplemens To The 1986 Northwest Conservation and Electric Power Plan, Volume 1. Northwest Power Planning Council, 1989. 
Conservation is also a uniquely flexible resource. Some conservation programs automatically match growth in electrical demand. Such is the case when new buildings are mandated by code to be energy-efficient. Each new building adds load to the electrical system. but can also save energy if it is better insulated than current practice. Thus, if the economy grows rapidly, the conservation resource expands quickly; but if the economy slows, the conservation resource automatically tracks the more slowly growing loads. Conservation can also be developed more quickly than generating resources when more electricity is required.

In other regions besides the Pacific Northwest conservation is being taken quite seriously by states and utilities. In New England the New England Electric System in partnership with the Conservation Law Foundation has begun a $\$ 65$ million dollar per year energy conservation program. ${ }^{1}$

In California the California Energy Commission is writing and revising California's building and appliance standards. The Commission also forecasts energy supply and demand, approves or denies the need for new power plants, and reports to the Governor and Legislature on statewide energy use. ${ }^{2}$

The State of Hawaii faces the same problem as many of these other regions, whether to build more generating capacity or to become more efficient. The 500 MW geothermal project will cost the state and its ratepayers an immense amount of money. Conservation and increased efficiency will cost much less and involve much less risk. Table 26 shows the cost per $\mathrm{kWh}$ of some simple conservation measures and the effect they can have on reducing energy demand.

The 866 million kilowatt hours saved per year converts to about 123.6 annual megawatts of generating capacity ( 866 million $\div 8,760 \div 0.8 \div 1,000$ ). This is about $25 \%$ of the proposed $500 \mathrm{MW}$ geothermal project. It consists of only five simple efficiency measures and costs an average of 3.0 cent per $\mathrm{kWh}$.

1/ Energy Efficiency and Least Cost Planning: The Best Way To Save Money and Reduce Energy Use In Hawaii, Robert J. Mowris, January 11, 1990. (See Appendix D in this report)

2) ibid 
Table 26

\section{CONSERVATION MEASURE COST/KWH ${ }^{1}$}

\begin{tabular}{|c|c|c|c|c|c|c|}
\hline Erriciency Mensure & $\begin{array}{l}\text { Added } \\
\text { Retail } \\
\text { Cost }\end{array}$ & $\begin{array}{l}\text { Annual } \\
\text { Electricity } \\
\text { Savings }\end{array}$ & Life & $\begin{array}{l}\text { Cost of } \\
\text { Conserved } \\
\text { Electricity }\end{array}$ & $\begin{array}{l}\text { Estimated } \\
\text { Number } \\
\text { Units }\end{array}$ & $\begin{array}{l}\text { Statewide } \\
\text { Saviags } \\
\text { Million }\end{array}$ \\
\hline$\because \cdots$ & $\$$ & $\mathbf{k W h} / \mathbf{y r}$ & Years & $c / \mathbf{k} W_{\mathbf{h}}$ & Thousands & Million $k W h / y r$ \\
\hline $\begin{array}{l}\text { R-10 Water-Heater } \\
\text { Blanket }\end{array}$ & 25 & 650 & 10 & 0.6 & 215 & 140 \\
\hline $\begin{array}{l}\text { Water-Saving } \\
\text { Showerhead }\end{array}$ & 20 & 310 & & 0.9 & 215 & 67 \\
\hline $\begin{array}{l}\text { Compact Fluorescen } \\
\text { Lamp }\end{array}$ & 12 & 88 & 68 & 26 & 1,500 & 132 \\
\hline $\begin{array}{l}\text { Heat-Pump } \\
\text { Water Heater }\end{array}$ & 650 & 2,280 & 10 & 4.0 & 215 & 490 \\
\hline $\begin{array}{l}1989 \text { Best } \\
\text { Mass- Produced } \\
\text { Refrigerator }\end{array}$ & 60 & 125 & & 4.6 & 292 & 365 \\
\hline $\begin{array}{l}\text { Average Cost of } \\
\text { all Measures. }\end{array}$ & , & & & 30 & $\therefore$ & \\
\hline Total Savings & & & & & & 866 \\
\hline
\end{tabular}

1 Energy Efficiency and Least Cost Planoing The Best Way To Save Money and Reduce Energy Use In Hawaii," Robert J. Mowris, January 11, 1990. (See Appeadix D in this report)

Table 27 compares the cost of these five conservation measures with the cost of adding $500 \mathrm{MW}$ of geothermal, solar/oil, and oil fired generating capacity.

Table 27

COST COMPARISON OF CONSERVATION AND 500 MW ADDED GENERATING CAPACITY

\begin{tabular}{lr}
\hline \multicolumn{1}{c}{ Type } & Cents/kWh \\
\hline \hline Geothermal 20\% Contingency & $10.97-14.71$ \\
Geothermal 30\% Contingency & $1149-15.43$ \\
Solar/Oil & 10.81 \\
Oil & $\$ .73$ \\
Conservation & 3.0 \\
\hline \hline
\end{tabular}


The cost of conservation is by far the least expensive of the five options. Geothermal is the most expensive being 3.6 to 51 times as costly as conservation, while solar/oil is 3.6 times as costly, and oil 2.9 times the cost of conservation.

The conservation measures mentioned here are only a few of those available. Passive cooling building design, efficient air conditioning systems, waste heat recycling and co-generation can all contribute to lowering energy demand and cost. Conservation in concert with an aggressive solar energy program can have a considerable impact on energy use. In Florida, like Hawaii, cooling is a major user of electricity. Passive solar design in new homes can, according to the Florida Solar Energy Center, ${ }^{1}$ can cut costs in half:

In Florida. energy consumption in new homes can be reduced by $50 \%$ through passive cooling designs that add \$2,000 to construction costs to typical homes, according to the Florida Solar. Energy Center. Because an average home in Florida consumes $12,000 \mathrm{kWh} /$ year, a $\$ 2,000$ investment can save at least 6,000 $\mathrm{kWh}$ /year over an assumed 30 -year life of the home. The cost of the conserved energy is about 11 cents/kWh (in constant dollars) compared with the average cost of electricity in.Florida of $8 \mathrm{cents} / \mathrm{kWh}$. The total investment can be recouped by savings on energy bills in less than 5 years.

The designs that accomplish these savings include siting a new house facing north (for cooling), painting the house a light color, using light colored shingles or roofing, and installing attic radiant barriers, wall insulation, double pane windows with a reflective coating, and awnings.

And for existing homes:

Increments of savings can also be achieved at low cost. A recent estimate by Lawrence Berkeley Laboratory shows that planting trees in urban areas is a cheap way to save air conditioning power. By planting 3 trees around a house to shade an air conditioner, 750 to $2,000 \mathrm{kWh} /$ year of electricity could be saved at a cost of 0.2 to 1 cent $/ \mathrm{kWh}$ (assuming $\$ 15$ to $\$ 75$ per tree plus watering costs).

The cost of conserved energy from low-E window glazing is currently $\$ 4 / \mathrm{MBtu}$, and, as the market matures, the cost is estimated to drop to $\$ 2 / \mathrm{MBtu}$. (When these windows saturate the market early in the next century they will save cnergy equivalent to one-sixth of the nutput of the Aluska sipeiine. or over 300,000 barrels of oil per day.)

1 $\quad$ Power Surge. The Status and Near-Term Potential of Renewable Energy Technologies, by Nancy Rader, for the Public Citizen Critical Mass Energy Project, May 1989. 
And for both new and existing homes:

The cost of energy saved over the 20-vear life of an active solar domestic hot water system is about 4 to 5 cents $/ \mathrm{kWh}$. These systems can save from $40-70 \%$ of annual water heating costs.

The performance of solar energy systems is continually improving along with their appearance, reliability and cost. And they are becoming more and more competitive in the energy market place as Richard Balzhiser of the Electric Power Research Institute states in the previously mentioned article from Nations Business, February 1990:

Solar energy could be an exception. Balzhiser says. I think solar and particularly photovoltaic technology [in which sunlight is converted to electricity] is one [area] where we'll see continual progress scientifically."

Much of that progress is already here and readily available.

The conservation option and the solar option are two extremely important ways by which Hawaii can reduce its future energy demand. If these areas are explored and promoted with the same zeal as the geothermal project they hold the promise of even greater benefits with much less cost, risk, and public agitation. Hawaii should consider establishing a separate state government agency simiia: $: *$ the Northwest Power Planning Council and the California Energy Commission to examine all energy issues, needs and options and to actively develop and promote the most effective and least cost of them. The newly formed Hawaii Energy Coalition, a citizens group of planners and environmentalists, seems already headed in that direction. 



\section{NEA - CONCLUSIONS}

Based on this analysis the $500 \mathrm{MW}$ geothermal project is the more costly and more risky of the available options. Its cumulative cost over 40 years in 1990 dollars is between $\$ 10.8$ and $\$ 15.7$ billion while solar/oil is $\$ 10.6$ billion and conventional oil is $\$ 8.6$ billion. In terms of net present values (the cumulative costs discounted back at a constant rate over $\mathbf{4 0}$ years to indicate how much you would have to invest today to achieve the same end in 40 years) the geothermal project is between $\$ 45$ and $\$ 63$ billion while solar/oil is $\$ 3.6$ and oil is $\$ 27$ billion. You would have to invest $\$ 0.9$ to $\$ 2.7$ billion more today in geothermal than solar/oil, and $\$ 1.8$ to 53.6 billion more than oil to achieve the same benefit over the 40 year anaiysis period. Table 28 shows the annual cents per $\mathrm{kWh}$ increase over the estimated average current rate ratepayers would have to pay to cover the costs of the various generation options if they were incorporated into the overall generating system.

Table 28

INCREASE OVER CURRENT RATES FOR VARIOUS GENERATION OPTIONS (cents/kWh)

(Estimated 1990 Average Current Rate-7.43e/kWh)

\begin{tabular}{lcc}
\hline \hline Type & Levelized Cost & $\begin{array}{c}\text { Average Rate } \\
\text { Increase Over } \\
\text { Current Rates }\end{array}$ \\
\hline Geothermal $30 \%$ Contingency & $11.49-15.43$ & $1.40-2.42$ \\
Geothermal $20 \%$ Contingency & $10.97-14.71$ & $(18.9 \%-32.6 \%)$ \\
Solar/Oil & 10.81 & $128-2.24$ \\
Oil & 8.73 & $(17.2 \%-30.2 \%)$ \\
& & 1.23 \\
\hline \hline
\end{tabular}


Ratepayers could annually pay more than three times as much for geothermal generated power as for oil generated power, and twice as much as for solar/oil generated power over their current rates.

In spite of the fact that geothermal costs are high NEA considers this analysis to be conservative. Recent revelations at The Geysers in California, the largest geothermal power production field in the world, indicate that the project is running out of steam and that billions of dollars may be lost as a result of this unexpected turn of events. This from the Oakland Tribune, November, 1989.1

The world's largest geothermal-power producing field, The Geysers near Clear Lake, is running out of steam.

To the astonishment of most geological experts. the steam that has powered $\$ 2$ billion worth of nearly new power plants is declining stiarply, and electrical output is dropping.

Over the past two years, steam pressure has dropped 20 percent; some experts now predict it will be down by half by the end of the century.

This caught all of the geological experts by surprise," Charles Imbrecht, Chairman of the California Energy Commission, said last week.

"We're taking it very, very seriously. There is several billion dollars' worth of investment in The Geysers," Imbrecht said.

The Geysers is the most studied geothermal reservorr in the world and the most developed, yet the predictions and theories concerning its energy capacity and potential are falling far short of expectations as the Oakland Tribune article goes on to state:

Since oil and gas companies operate The Geysers steam wells, their officials are especially worried. Tom Sparks, a geothermal expert with Unocal Corp, the largest Geysers developer, said, "No one foresaw this happening.

"We had thought there was a steady boiling mechanism 15 miles down, but that theory isn't working," Sparks said."

1) Geysers Failing, Billions of Dollars May Be Lost, By Steven H. Heimoff, The Tribune, Oakland, California, November 5, 1989. 
The owners and investors at The Geysers are now rethinking their position in light of recent events:

Eastbay utility customers as well as investors have a stake in The Geysers.

Geothermal power is cheap because is uses free steam and a simple generating system, if it peters out electricity from more expensive sources will be used and The Geysers idle power plants will still have to be paid off.

Pacific Gas and Electric Co, which dominates the region with 19 power plants, has cancelled plans to build two more plants.

If this could happen at The Geysers, an area which has been studied so thoroughly, it could easily happen in Hawaii where the resource has been studied little by comparison.

Geothermal experts in California now feel that the original resource was overestimated and that too much steam is being withdrawn too quickly. Again, from the Oakland Tribune article:

"Geothermal power is a depletable resource. It's been known that the field would decline" said Unocal spokesman Harry Bain.

"Many of the plants were built in the middle 1980 " energy commission information officer Claudia Barker said. "They should have a 20 - to 30 -year lifespan." Instead, she noted, they may last half that long."

Experts can only guess at the reasons for the shortfall, but most feel that too many plants are tapping a resource that is more limited than originally estimated.

The Northern California Power Association, a consortium of municipal utility companies, testified before the State Energy Commission on Sept. 21 that the problem "is directly related to the mass withdrawal of steam."

PG\&E public relations spokesman Dick Davin agreed, saying, "There are too many straws in the soup.

The proposed Hawail Geothermal Project will require 30 production weils (straws) at $4 \mathrm{MW}$ per well and 400 production wells at $3 \mathrm{MW}$ per well over the expected life of the project at $100 \%$ replacement. If, as is the case at The Geysers, the resource rapidly becomes depleted and the wells fail sooner than expected more wells will have to be sunk to try to replace the lost energy. At $200 \%$ 
replacement the number of production wells could be between 450 and 600 over the expected life of the project. This large number of wells may easily overtap the reservoir.

For sake of continuity our analysis assumes that wellfield production will be adequate to maintain 500 net MW of output throughout the analysis period. In reality, however, this may not be the case and costs will rise accordingly. (See Appendix E for project costs at $200 \%$ well replacement.)

The extent and potential of the geothermal reservoir on Hawaii is unknown. For the most part it is being assumed that the $500 \mathrm{MW}$ ( $600 \mathrm{MW}$ gross) of energy is there and will be available for the long term. But according to testimony given in 1982 by Robert Decker. Scientist-in-Charge of the U.S. Geological Surveys Hawaiian Volcano Observatory?

Any electrical power extraction from the Kabaule'a section of the east rift of Kilauea in excess of about $5 \mathrm{MW}$ will not be replenished by new thermal power from the volcano and will probably deplete the geothermal resource.

The simple fact is the experts do not know how large the geothermal resource in Hawaii is or much energy can be extracted or at what rate before depletion occurs. Until this is known, rushing headlong into an incredibly costly $500 \mathrm{MW}$ development project makes little economic sense, especially when other alternatives like conservation, solar, and improving existing erticiencies are available at far less cost. The geothermal project is being touted as a means of putting an end to Hawaii's energy problems when in reality it could be just the beginning of them.

If the state of Hawaii is really concerned about its long-term energy needs it should begin by looking at what an aggressive energy conservation and energy efficiency program can do about reducing energy demand, and then examine its least cost generation options. A single massive energy project is not the answer for the long run. because in the long-run survival does not necessarily go to the biggest ur strongest, most of ten but to the smartest, most adaptable and most efficient users of resources.

1) Energy Efficiency and Leas Cost Planning: The Best Way To Save Money and Reduce Energy Use In Hawaii, Robert J. Mowris, January 11, 1990, (see Appendix D). 
APPENDICES 


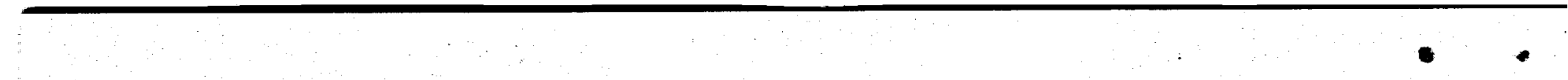

\section{APPENDIX A}

WELL COSTS 


\section{molst stemets}

fiell eilling forts

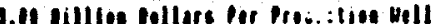

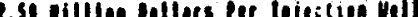

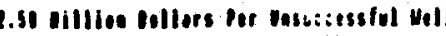

II in per eloet vell fiele

I win per will

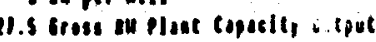

25.1 int ou flost copocity e.tid

2.5 in Hoot leserve capocity

2.3 In Wellliele mesesve lafo:it,

11 eredetlon yells

1.0101 .1 Productionflijection vetl titto

11 Infection vells.

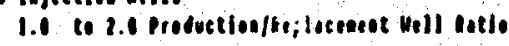

a Predoction Inflacronet hills

lisectles liplacioent Hells

1.1 Bt 1.1 vell orlllin; Sacsess lotio

31 ensucesssfol wells

"I total uells lepulese over life if ploot

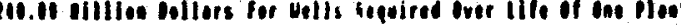
11 eneler of Pleats

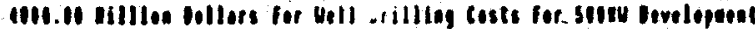

(II) Tolit Prefuction vells

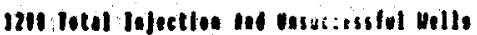

ilit totol mills

It sctulitio

Hell fiele orllilog cort

.11 Billion Dellors ler probuttion Mell

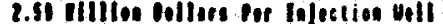

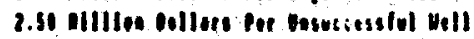

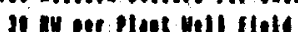

In per will

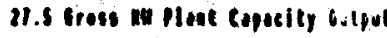

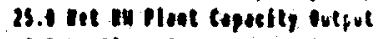

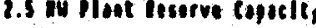

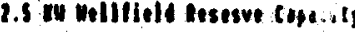

10 Preductlon Dells

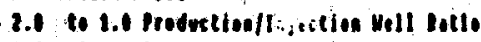

sinjetlon Wills

1.1 Io 1.0 Production/le;

10 Preductlon enplocionet tull

Hejoetlon Replecenest vells

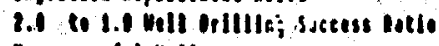

11 Monetersfol mellis

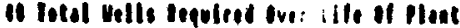

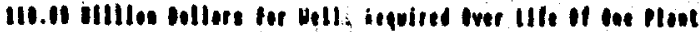

21 Iunber of Pleats

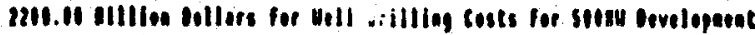

iII Total Broductlon Holls

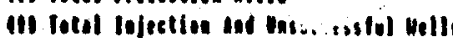

ili letel vills
Its cost

Vill fiold urilling Costs

2.35 IIllioe Pollors Mer Production Well

2.11 Itllion Elllers Per Iofestlon Vell

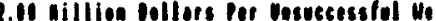

31 In per plone vill blese

3 in pIr Well

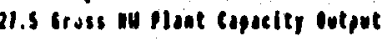

H.t Het in Plont tapocity entgut

1.5 wo ploat Eeserve copictey

I.I Wh Hellfiell Resesve sipacity

11 Production Vells

1.1 to I.1 Productlon/tajection Wall hitle

IL Injestion Mells

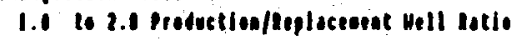

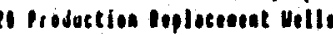

21 Ia jectloe Eeplocesent uolls

1.0 to 1.0 vell billling secerss hatio

JI Unsuccessiul Velli

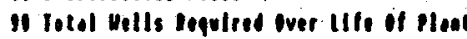

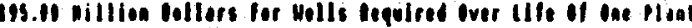
21 Rueber ol plients

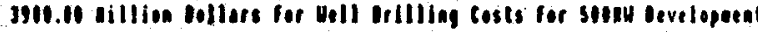
ill lotal Proluction Vells

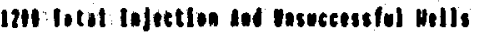

IIII fotal vilfs. $\therefore$

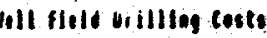

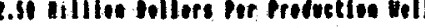

2.11 hillion tollere Ber hajeetion well

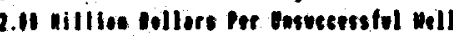

nut per elont will fiels

I ner peritull

21.5 Gross an Hont copocity output

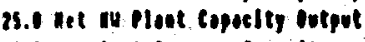

1.5 Im. Deat Reserve topectty

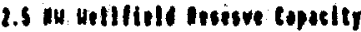

11 Production Nills

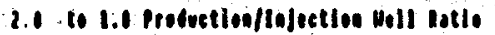

I Infortlon mells

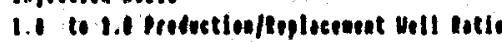

11 Irojuetlon Eeplocreset Wells

5 Injection Replocenot Halls

2.1 to 1.0 Well Ellliting suecoss 20110

II Dusuctessful Mells

1I Total Nells Aepulese ever tllo of plent

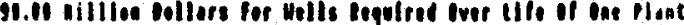

31 luaber 11 Ploats

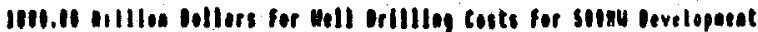

III Iotal Production Malls

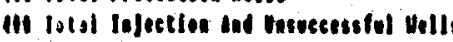

III Int, Mells
IOW CISI

Hell fiele frillion costs

2.11 illlen Dollers Per Production Well

.50 milliee Bellers Per Injectlon Hell

1.50 IIIllen Dellors Per Insuecessful Wetl

30 wh ger Blant velt flele

In pier vill

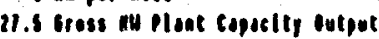

25.1 tet in pleat capoctey dutpet

2.5 ny Plont leserve capacity

2.5 in Vellfield desesvo copocit

11 production Bills

1.0 to 1.1 Profuction/folection Well lotlo

11 Injectlon Vells

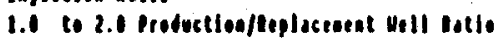

21 Productlon Ieplaseneat Wills

2O Injection Inplecenent Uells

1.1 te 1.1 Well billilag Success lotio

31 Unsuccesstul Wells

91 lotel wells lepulect over tile of pleat

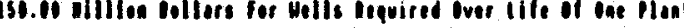
21 Ineber It Plants

3111.01 Aillion Dellors for Mell Brllling costs for sulu Developant

ili letal Productlon Wills

I211 fotal Infretion dif Vesecesssful Mells

IIII Iotul beils

\section{ton tost}

\section{Whition orition costs}

.m nitiven Deilfers me production vell

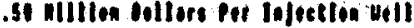

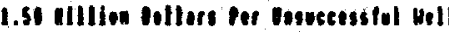

16 in pert piont well fiele

in weer Well

21.5 Gross w ilout copoctity outgot

25.1 hit an Plant copectty entpot

2.5 m plent Reserve tepaclty

2.5 en Nellffese Deserve copecit

11 Broduction Nells

2.1 to I.t Prodection/lojection Hell Rotio s Injection Melts

1.1 to l.0 Prodection/feplaceent vell Rotle

o Preduetlon Inplocesonet wills

Iofoctlon Reploceont Mell

2.1 to 1.0 Gill Ifllting succels totlo

18 Uecucensifol velli

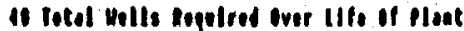

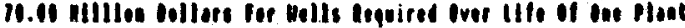

20 Ineler Il Hidets

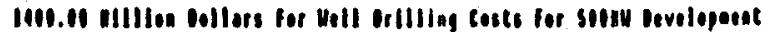

III Total Predectlon Mells

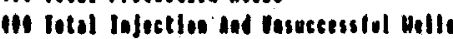

III retel Nells. 
HSt StEIIII

\section{IIGI cosi}

Well flele billileg costs

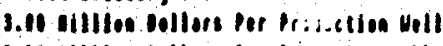

2.51 illtion bellere ter tapectien vell

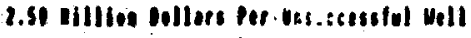

It il jor hlane vell tlets

I nit per uell

21.5 fress on Heart tapacit, sutput

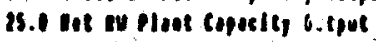

2.5 in Ploit Resorve topacits

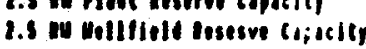

it Predection velle

3.1 10 1.1 Prolection! iajection bell totio

I Iajestien mills

2.1 to t.1 Broductioa/itplactacet Mell Batlo

S Produetion Beplassent -ills

2 Iajestion Inplocesurt bells

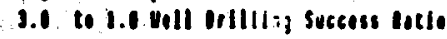

5 Vnevecessful Molls.

25 Intal Velle tequired o., ; ilfe of plant

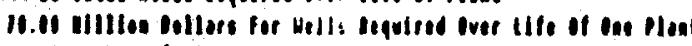
21 eneler of pleats

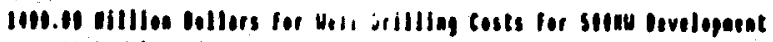
3il Total Peoductlon Wells

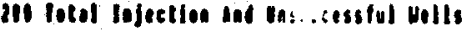

iti lotal vells

\section{III cest}

Hell Hiels orillies tests

2.51 Billien bollors per Prodectlon Well

2.11 aillion bellers per Injection vell

2.14 nillien Bellore lor vesuecessfol vell

30 en pee Plont Will fiele

$$
\text { J III per Wall. }
$$

21.5 Gross all plost capecity output

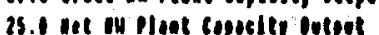

i.s in pleat peserve capocity

2.5 an vellfleld lesesve topecity

11 Proluetion Mells

3.1 to 1.0 Profuction/hajection Well lotic

3 infection tiells

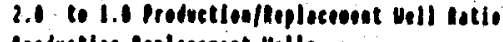

s Production Ieplaserest Hells

2 lajectlon inloceset tells

J.1 to 1.0 Well brilling Success totie

s Unsuctessiful Wells.

as rotal Vells Repuired ever llfe if elint

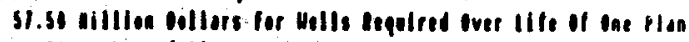
21 inebie of Ptoats

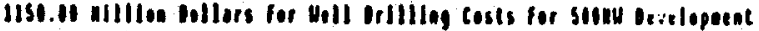
Jil Total Protuction Wells

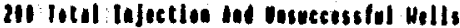

sil local Wells
LON COST

2.11 Alllion Dellets Per Productlea Mell

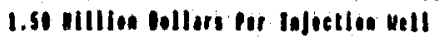

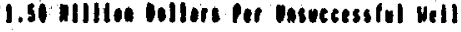

II tu per Ploat Mell fiele

$$
\text { ond per Vill. }
$$

21.5 fress all plact cancelty output

25.1 Het in Plont copostty outpit

2.5 win Hont lowerve topacity

2.5 an vellftele teseive topacity

11 Production Uolls

3.8 is 1.1 Irotuction/Injection Well litio J Injection Wells

t.1 to 1.0 Reductlon/heplaceoent Hell Iotio

s Provuction Reploceseat vills

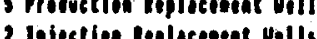

1.1 to 1.0 Mell irillian success cotle

s insutecsiful vills

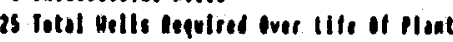

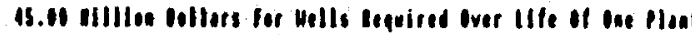
21 Vuaber of Plonts

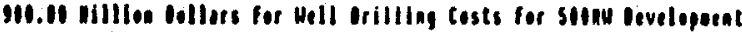
311 Total Production vills

2il Totol Injectloe And Unsuccessful Mells

sil Totol Vilts 
unst setIIIII

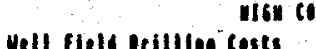

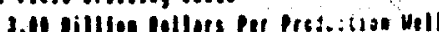

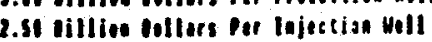

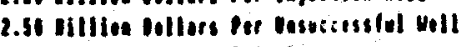

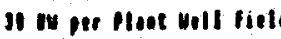

I In per Vell

21.5 lross on Plont copuelty : ajut

25.1 Int in blot tapocity bit,

2.5 In pleat teserve caposit,

i.5 on inellfiate tesesve coperits

Productloe belli

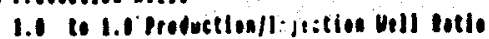

1 isjection vells

1.1 102.0 frofuction/he; ilecent vell estie

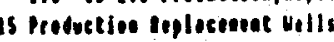

is Injectloe inplosenest vells

1.1 It 1.1 Woll Irillian sicess tatio

23 insuctessful vells

Ge letel vells tequired ever icle of llont

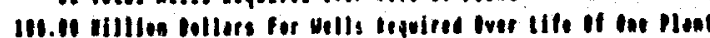
21 neberer 11 Heants

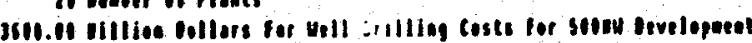
ist lotal probection vells.

III total Injectloe and vasucitisful vills iss totul Hells.

II strinio

\section{vitin cost}

vell fins infling costs

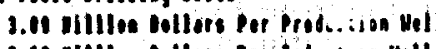

.31 ifllion Dellats Pei tojaction vell

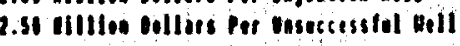

II en por plont vell flelt

I in per vell

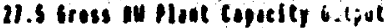

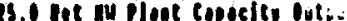

2.5 me pleat teserve copecte

2.5 we vellitiels lesesve cape it

productlon tills

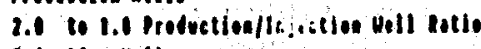

1 Iolection Mills

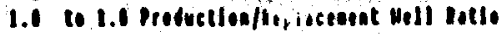

I Moduction Ieplecenent vells

- Iojectloe ieplocenest wells

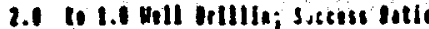

a Desuecessfol Mells

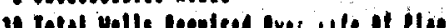

ex.so million bollors for velts nequires over tife if one ploet 18 Pueber of nlats

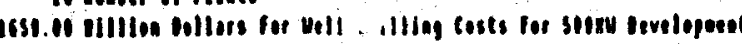
jil folit Productlon Millo

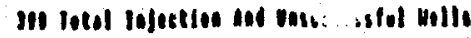

(1) Tocut vells
III cast

Well flile irilliag costs

2.51 Aillion Dellers Pes Production Dell

i.l1 Billiea collers Per tojection bell

C.II Aillion Iellars Pei Unsuccessful Vell

31 en per Plant Noll fies

I IN Der Nell

21.9 Briss in Plont tapaclty entput

25.I let on Plant Capotity Dotgut

2.5 RH Ploat Retervi capicity

2.5 It bellfleld lesecve Copactey

I Prudectloe Velle

1.1 Io t.1 Protuction/landection well latie

I Iajectlon vells

1.1 to 2.1 Proluctloo/feplocenent Mell Retio

is Production leplecesent Mills

is Injection leplacement villi

1.1 10 1.1 Well orlilling Sectess Ratio $2)$ Hisutcessful Mells.

It letel vells legulred ever tlfe of plat

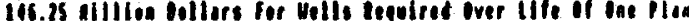
21 Analer of Plonts

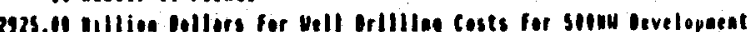
ist lolot production Vells

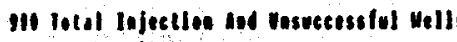

lost total vells ;.......

ill cos

Hell fiefle orilting costi

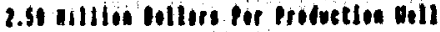

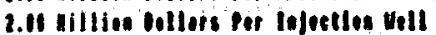

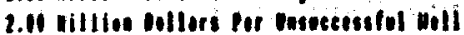

of in per pliat well flet

Int per vell

21.5 Gruss ett Hant copecity output

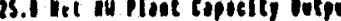

2.5 nt plost Reserve caposity

i.5 wa vellfieft teseive coposit

i pirduction vells

2.1 to t.o Prodictionflofectloe Well hotie

1 Cojectlos Vills

1.1 II 1.0 Production/eplocenent vell hatio

a Poductlon Riplocenent Mells

1 Ia jectlon leploeserat thells

2.1 10 1.1 Woll erilliag sucerse Rotlo

Dinsuccesifus vollo

31 lecal vells tepolmed over tlfe of hloat

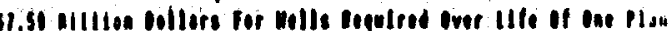
21 isclee of plonts

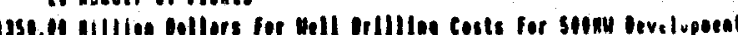
ill tilst Prevection Welle

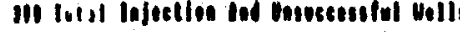

III livial vilts
IOII cosi

Well fiell irilling costs

2.11 Allles bollers Per Profuction vell

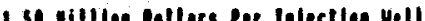

gillies bellers Pet Desectesslal vell

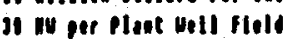

I in per vell

21.5 sesses nu Hant tapocity butpot

25.0 Iit an Dieat sipucity outpot

2.5 in Ploat leserve copactly

2.5 Nh Welllitld Iesesve tapocity

1 Pioduetion Mells.

1.t to L.e Pinduction/lajection Vell potio

- Inlection Mells

1.1 to 2.1 Preductlon/leplacesent well tetlo

is Protection enplocenent Mell

is Inlection ieplicenent vells

1.1 to 1.1 Wall Drlliting success hotlo

23 Vesocecssful vills

ce Iotal Hells kepulred over lite if plast

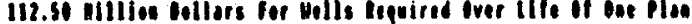
28 Nueber ef itonts

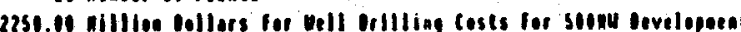
ise botal Preluction mells

in ietel injection mad resuccessful Hells IIsI totil vells

Hil flele Dillilag costs

2.11 Alllios Bellers Pee Productlon we il

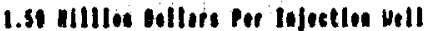

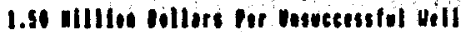

11 an per plinet Hell titel

I new per vili

21.5 Grose an pleat copocity onsyut

23.1 Het in Bloat Copectly lotgol

2.5 nu bloat linorve capicity

2.5 nh Wellffell tesseve tepecit

1 Production Vells

2.1 to 1.1 Productionfinjection Well lotio

I injection Mills

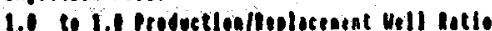

- Production tepleceecent Vills

1 Jojection inplosoment Hells

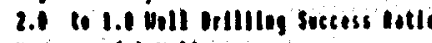

- Vasuceesiful Mells

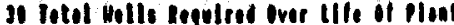

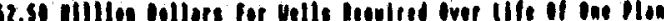

II Nuaber Ol Plants

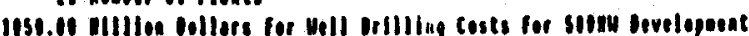
Jil totel Production Hells

III total In Jestion ind Insusetisful Nalls

ili Votal vilis 
itsl sctinis

Well Hels orillisg ents

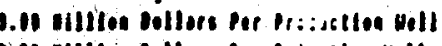

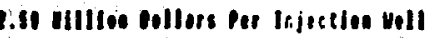

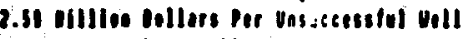

II en per Dlost Uill flits

1 nu per will

21.8 coses In Host copocit, istent

as.I IIt nil noot copecity 0.tput

1.5 an llat Peserve copacity

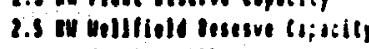

incoluction Helli

3.1 to 1.0 Pinduction/lijectlos vell Rotio

1 Iojectlon Wells

2.1 $10 \cdot 1.1$ Profuctlon/iaslocesect Well lotio

e Production inplocemeat Helli

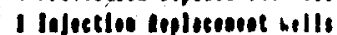

3.1. to t.1 Hell inlliting Sucters tetie

Insucessiful vells

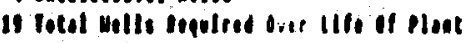

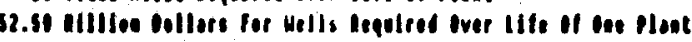

21 lueber of plasts

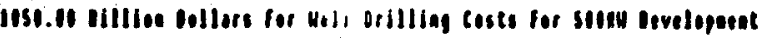
225 total Pceduction Wills

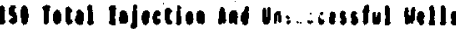

Jis Total Walls
III cost

Well fiele orilllas cests

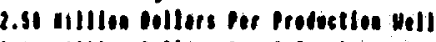

2.11 sillioe Bellers per tojection vill

2.11 Hilllon Dollers Dor besucetssfol Well

JI In per ploat well flete

In per Vall

21.S Gioss in Plinet copocity outgut

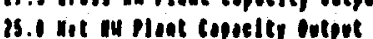

i.s an plout leserve copoelty

2.5 en vellliell Insesve capocit

1 Produetion Mells

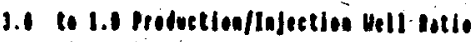
J Injestlen Wellis

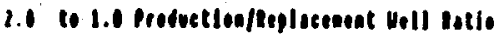

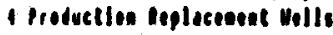

I lajectloe lieplociesent Vells

J.1 to 1.0 Hell filliling Socteis lotle

a Unsuccesiful vells

19 Volal Mells Ineuted ever tlfo of hlant

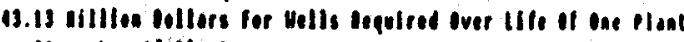
21 iveleer of Plante

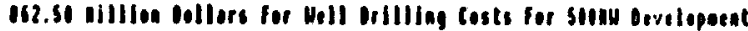
22s lotul Production Volls

ist lotel io lection ind tesuceess fel vells

jes local wills
III fiell orillion corts

2.11 alition Dellere her production vell

1.51 illilion Dollers Per Injection Well

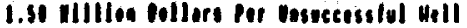

II in per Pleet vilt fiels

I nin pir vell

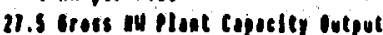

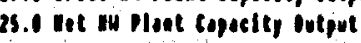

2.5 wh plont enererve copestly

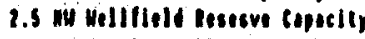

e Productlon Vills

3.1 to t.t Production/lajection vill tatle

J Injettlon Wells

2.1 to 1.0 Preduction/liplaceoent well hetlo

e Preductlon meplocenent vills

I In fection Replocenent Wells

3.1 to 1.1 Mell ofllling success Latio

insutersilus vills

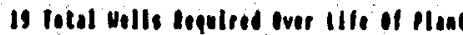

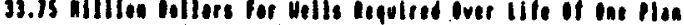
21 Unetel of Honts

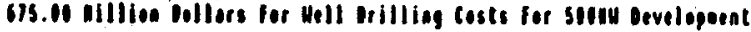

225 Jotal heduction Nells

ISt lotal lajectlon ded Unsuccessful Wells

IIS Total Wills 
APPENDIX B

PLANT COSTS 


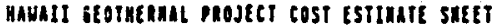
is xet afidutt hant

Lot tstinult--1958 vollars

ecsontee teapentures:

PLANT Caphestr:

ietrrocosy (SF, 0F, I):

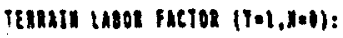

igtuin site MeP $(\mathrm{T}=1, n=1)$ :

corsteyersor cant $(7-1, n=1)$

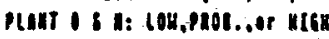

Hoovetion Eotment: $1,1,8$

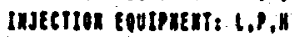

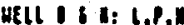

Electutetir cost:

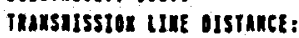

Renaturas assessaent HoRt

Pentits/lustuses:

I Hooveriar uztus:

1 Jisecizor uelts:

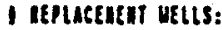

I visuccessful MELIS:

HELIFIELO CAPITIL COSTS Basobction coulbuter

Eusction toutpatrit

iEalaIn Labor hojusthent

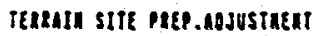

construetion cant

SO TO T I (UELFIELO)

SOITOT I (UELFELI) Helthite tost/an HELIFIEL COST/RH

UELI \& HEILIELI O I I COSTS

HELI \& HettrteL 1 I I costs

poute phat captral costs

pouet pturt

Mas matenent

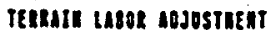

TERAII SITE RREP. CoJystuent

constaperion taip

S I I I I I I (BouEe Plant)

S y 8 I O I I L bouer mant) d duet plant cosicha

nant os a cosis pouse plant cost/Re

puat o cosis

PUAT/UELtHEL EAPITIL Costs PuIT/UELIIELI cost/at Mutr/uectfiels cast/5y

neIl ortilung costs

Ithrsaisster tiuk costs

Totat cartut tosts

IOTA 18 a costs

pherputh onse cost:

Iruer cosis:

Iorat nurt/uell tase cost:

UtIu zet contrnester:

tauger of oguer planis ve:jeg:

rOTAC COST fOR POUEL PLMIS submaine casie costs:

ovetneno tenishisstor costs:

total moject captiat tosi:

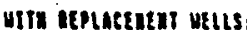

mayal mosect o I cost:
358 ate 6

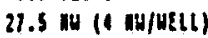

SF

1

13 ceats/km

11 siles

2.56 eillioe 1991 \&

1.15 aillion 1956 ;

8

1

18

1.90

1.11

1.11

1.19

1.15

16.93 aillien tont

11.91 eillios 1990 :

C.13 oillion 1994

1331931 ।

0.74 alllioa 1981 \&

c.86 sillies 1934

21.31

5.10

..11

0.62

2.15

34.21 cillion 1964

37.32 aillion 1990

a.ds cillion 1994

1357 1395

1.12 sillien 1948

1.98 eillion 1998 i

19.23 allliea 1991

1.19 eillioa t9st :

IISt 1395

13.15 sillioe 1939 :

3.61 eillioa t991

c.58 eillion t99!

2.11 alllion 1991

16.58

3.25 cilllon IS9)

19.03 dillion 1991

111.14

2111

3155.11 atlion 1998

314.56 aillion 1990

298.94 eillieg 1998 :

2823.31 oillion 1990

3635.31 silllen 1950

55.11 sillloe 1931 t
MAUAII GEOTMERHA PROJECT COST ESTHAIE SHEET is net aesauti nhat

HItR esfinaft--1990 Botcats

tesomice renperiture:

plati capactir:

recnuotost (SF, DF, a):

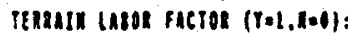

"inats site inep $(Y=1, n=0)$ :

Constanction canP $[\mathrm{Y}=1, \mathrm{l}=0)$ :

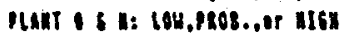

Propuetion EQULPIEXT: L.P,

Iusetrox coutpuent: $l, P, n$

HEU । I I: l, P.

etettiteItY cost:

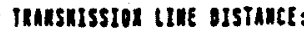

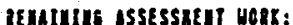

penitis/lutensts;

a nopgetion wetLs.

I Injection uetls:

IEPLEEREI MELL:

I utsatcessfol uells:

51.5 an (3 na/UELI)

si

I

1.

H

.

13 ents/klth

11 eiles

3.11 Eillion 1990

1.11 silllog 1990

5

I5

10

MELIÉEL CAPtIAL casts

meovetiox gQuiphent

: raection EOUtPaEnt

: :zatK Lago hojustaent

itanilk site past. nojusinext

consinoction chat

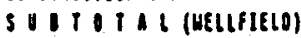

S I I I I I (HELLIEL) UELIFIELO COST/WH UELCFIEL COST/LY

UEtI \& UELCIEL I I COSIS

uELI \& utLIfLI \& a costs

6.13

$\therefore 2$

1.11

.29

1.16

13.15 sillion 1984 ;

14.32 silllon 1991

0.52 sillion 1990

521 1951

1.11 sillion ises

1.19 Eilllen 1930 i

poute pany capital costs

Pout Plati

u2s mutsient

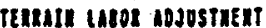

retrain stit paep. nojostagnt

constnvetion cast

S U I I I I (Poute Plant)

S I I I I I (Povel Pant)

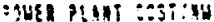
poute plant cost/Ris

plati o costs

clant i cosis

plant/uectetete captral costs DAMt/UELIFIEL COST/MU PLART/UEtIfIEL cost/RH

uetl otrtutus costs

inatsission tIIIE costs

torat capital costs

Toril 1 costs

pouke plati gase cast:

oinet costs:

roril pouse plant case tost:

utth 218 conttheznes:

uget if gades zints vesgeas:

otal cost fat pouet piants: subaritue chale costs:

ovenntao transuission costs:

TOIII PRofet CAPITAL COST: uIII IEPLACEnEI UELL:

24.34

9.10

1.11

4.68

2.85

31.57 aillies 1984 \&

40.11 cilllen 1990 :

i) 11:.2: :::1:

21881331

3.12 sillion 1981 \&

3.62 eilllen 1994 \&

55.23 eilltos 1994 : 2.01 sillion 1990 : 2018 1998 8

is.08 eillios 1998

3.60 stllion 1991 :

113.13 eillion 1934

1.01 allliog $1994:$

113.13

1.00 ellllea 1994 ; 111.03 sillion 1991 s 129.11

$\because 11$

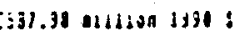

314.56 eillien t191

296.94 aillion 1998 :

3261.At nilllen 1996 t

131t. As alllion 1998

Annout nosect o I cos?: 


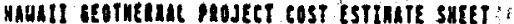
25 MeT MEshuth Plant

Lon Estinhte--18so gothats

agsontce IthPERIVRE:

punt caphetty:

teculolocy (SF,01,0):

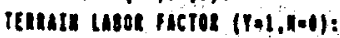

"BRthil sIte MEP (Yol,kel):

sonstasction cakp ( $P=i, n=1)$ :

plurf o \& a: tou,pros. of nies

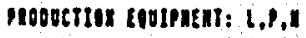

tusection tevipueni: $1, P, \mathrm{n}$

HELI I I H: l, t,

Eletintcitr cost:

renisuisstox tIAE uistaxes:

aenimuths assessuent wort:

PEInits/licensts:

I Roouctiax uELis:

I insectior uzus:

I EtplacenenI uEuls:

- I menceessfal netls:

HELFHE capinat costs

poovetion EQUIPEEI

Injectiox EOUIBuent

Egreain carof aosustueat

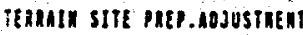

construetion canp

SOIO 1 I fueltheto)

S I T T I I (UELIFIEL) MEttfHEto cost/Ry UEthIEL COSI/Ru

WELI I UELIIIEL O I I COSTS HELI \& MELCFIEL O I I costs

Poute Dont capital cosis POUE NATt

nis Msifenent

imail tasue nojostuent

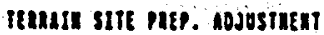

constavetiox cans

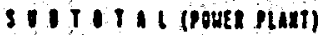

g i t i I l fouke puat) gure buart cost/na gousi noax cost/in

plant o a cosis

nuit 1 is cosis

NurfiuttfLets taptin costs MUIT/UELBRELI COST/RH MIIT/UELIIELII COST/ti

uEll oustims costs

Inisnission IIIE cests

fortl cantiat costs

iorit I I 1 cosis

MatiJuEL UASE cost:

oruet costs:

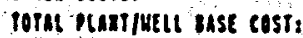

uttu 36 corrinstuer:

nunese of peuen plants ietoed

ijhi cost rot poute planis: sysmetie casic costs:

ovetaten inasxission cosis:

Total Mosect caftiai east: uIfn atnuctuitt uells:

itubut mosect i s cost: sst oes 6

21.5 at (1 andUELI)

Sf

1

$\therefore$

$\therefore 1$

1

$\therefore$

13 enals/huh

10 slles

2.51 dillion 1990

I.IS alllion 1934 !

1

4

12

1

1.96

1.78

1.11

1.19

1.16

11.93 sillion 1941 s

11.91 aillion 199t

0.13 aillion igge

4331999

0.71 ajllion 1984

ovi aillon 1994

24.31.

6.16

1.11

i.62

2.15

34.21 eillion 1921 i

37.32 aillion 1990

1.36 aillion 1990 i

1357 1930 ?

i.12 ailliea 1984 ।

I.II alllion I998

13.23 aillion 1931

l.Ts ailllos 1991 IIs) 14948

J3.15 ailllea 1991 \&

J.60 aillien 1931

ch.se allllon t934

2.78 ailllon 1991

86.5

3.25 nill1on 1951

19.03 alllion 1994

II6.11

tin

235s.16 oillign 3931

314.56 eillioa 1990

29t.g1 sillion 1994

givs.96 villion 1998

3t86.16 aillion 1994

ss.11 alllien 1990 ।

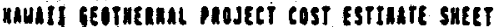

as wet nesauatt nant

HIte Estinate-19se ootlars

Itsonet tenpeantuRE:

Next capaetry:

iccunotost (SF, af, D):

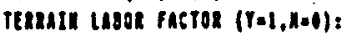

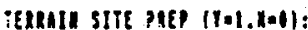

constuverion canp $(\gamma+1,1=1)$ :

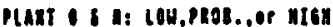

reoucrion eouinent: $L, P, n$

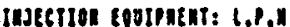

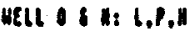

cleetiteity cost:

tmansutssior that otstance:

Memintus issesstent work:

venits/ticenses:

I nopertist hetis:

Insection uelts:

- Meptacenent vetus:

- unsuceEssfut utLs:

358 OES $\mathrm{C}$

21.5 II (3 WH/WEL)

SF

1

I

1

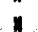

11 ceats/tht

11 iles

3.01 aillion 1994 \&

1.11 oillioa 1931 ;

it

5

15

HeILIEH CAPITH COSTS

poovertor equtpentit

insection covibnent

- Exhin cheol cojestuent

izanaIn sITE rep. hojustuent

construetron canp.

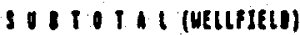

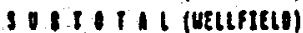
UELIfIEL CAST/IN uetifiets cost/R:

UetL 8 uethfte 1 a tosis

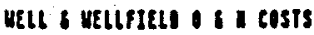

poues Dant capstal costs

pate nant

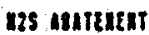

IERAIn lasol aojestuen

teruti stit paEs. cosostinent

censtiverter casp

50 I 1 । I (Moues puat)

s o i i a l (Douer plant)

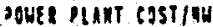
puEa DAnt costite

nuit : a costs

plati in costs

nAT/UELIFIE CAPITI COST ptatr/nEtLFEL GST/NH PULT/MELFIEL CIST/RU

UEAt ORIIIIU costs

tutsursstor llue costs

Poral caltal costs

TOTII \& I cOSTS

6.13

$\$ .12$

1.11

1.21

1.16

11.15 allliea 1984

11.32 sillien 1990 i

1.52 elllion 1994

$\$ 2119908$

- 1.10 allllon 19848

1.19 alllea 1990

11.24

9.11

1.11

1.68

2.15

31.31 alllion 1941

18.91 cillion 1991

C.19 aillion $29 ! 1$ :

ilss 1394 \&

3.32 silliea 2984 \&

3.12 alllea 1994

55.23 allllon 2591 \&

2.11 afllion 1930 ?

2111991 \&

15.11 cillion 1994

1.61 aillion 1990

163.83 alllion 18s4 \&

t.t1 alliden 199t

poues plant base cost:

orter casts:

107al pouet nati lase cost:

utir jes contungency:

Wuger of POUER -PlantS HEEDEO

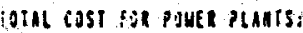

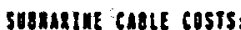

overuen thanshission costs:

rotal prosect captTal cost: WIIL eEpicenent uELIS:

innot nesect o i cost:
183.83

t.01 aillien 1998 101.13 alliton 1994 141.18

$: 1.11$

:313.j5 114ton 1:10:

J11.56 eillien 1590

$29 t .94$ cillion 1998 ?

3411.15 allilon 1994 :

4611.15 elllion 1990 ?

96.25 silllen 1996 I 
hawait feoryeaxal moject cost estrmate shet So aEt aecandr plant

LOW Esthinte--1981 DoLlats

ResonecE IEnPETHUR:

Punt chenctit:

TeEnoloit (SF,of, I):

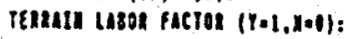

remuin sIIE neP $(p+1, n=1)$

corstevetiox Cane $(Y=1, n=1)$

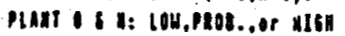

hoovetion Eovisnent: $1,8,1$

insection couthatat: $1, P, n$

WEIL I \& $\|: \ell, P, 1$

ELtThttin tast:

mansutssion the oistarce:

Remintus assessuent verz

nemits/licenses:

I Moovetior uelis:

i Insection hells

I Remucenent hetls

ansuctessivi utus.

UELHIEL CAPITAK GOSTS

peovertion sautrustr

sajectuan couiment

igarair lacor aojustuent

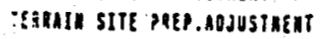

coxshuoction caxp

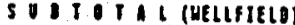

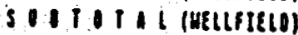
UECLFIESI costint HEUFIELO COST/RH

HELI I MELIFIELI O I COSTS

HEL \& MELCFEL I I COSIS

poute rant capital costs Pouet plat

L2S Muteat ot

retuit tasor nojostaent

IELIII SIIE MEP. ADJUSTHEN

coustauctiun cant

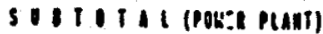

S O TI I I (POUEE Matit) pouse mint costion pOHER PLAAT ESST/RH

punt I I I costs

plati i I I costs

PGati/MELIfIEL capIIII costs nURT/UELLFIEL COST/WW

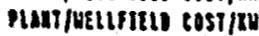

uEll Miture casts

Tuastissior une casts

crat coptrat costs

ma os a cosis

nlart/uell anse cost:

orute cests:

totul puat/uell onse cest:

uIt) 201 contunency

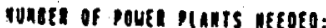

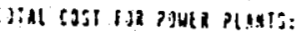
SURheral cade cosis:

oveaneas mansulsstor costs:

ithe moject capilat cost: virl Renactrent uflls:

antout mostct I s a cost:
358 ate 6

35 내 (4 Du/uttL)

sf

I

1

1

?

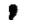

13 ecats/thth

10 siles

2.S1 aillion 1990

t.75 sillion 1999 :

16

1

21

9.19

1.11

i.11

1.35

1.65

18.56 alllioa 1984

21.38 aillion 1991 :

1.39 aillen 1998

3111930

1.52 ajllies 1984

1.65 ailllea 2998

39.51

11.31

0.11

1.01

4.68

55.32 cillien t311

60.21 aillion 1991 ?

l.11 aillion 1951 :

ills 1991 \&

2.94 sillien 1946

3.21 elllien 1998

11.54 aillion 19318 1.18 elllioa 1991 14831994 !

ci.se cillice 1994

3.60 cillion 1990 :

152.64 alllea 1990 s

T.As alllios 1998

152.64

3.25 afllim 1994 IS5.81 Eilllon 1954 : 111.11

19.11

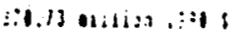
$3 / 4.56$ eillien w9t

298.94 cilllon 1931 ।

2511.23 nillisa 1930 !

3351.23 eillion 1991

11.54 eillion 1994 :

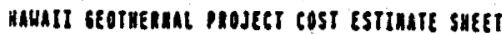

st det teguatt plat

AIE ESTIMTE--1990 00LMES

Lesouge tenperatgR:

358 ate $c$

ati capserty:

iecunotost (Sf, Af, 3):

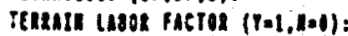

:EtraIn sIIE pite $(y=1,1 * 0)$ :

constaverion thap $(1+1,2=0)$ :

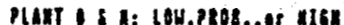

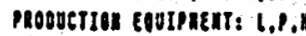

instetion foustent: $t, p, 8$

HELI \& a: 1,8,

cLeTRICIII cost:

imistissitx tIME eIstance:

IEmintus asstssuent woR:

ILUITS/LCEnsES:

I Proovetiox uells:

1 Injectioi nells:

I IEPLEEERT UELLS:

I unsuecessfot uelts:

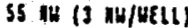

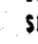

in (3 ha/ukti)

sf

:

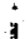

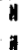

.

d] ceats/tub

10 siles

3.16 elllion 1950 ।

1.01 elllion t991

2.

II

38

MEtHIEL CAPITA tosts

pegagtion equipient

[nJECTIOR EQUIPIEXT

?Eanall labos mojustheat

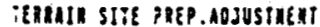

constauction cahe

SU:T I I I (WELIFIEL)

Sol10 1 1 L (HELIEIE) uettrizte cosi/hn Hettrigte costitu

HELI I veLIFIEL O I 1 costs

UELI I UELIPIEL 1 I COSTS

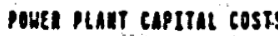

nute runt

nas mentenent

IEmiin LA80 nojustuEnT

temait sIte MEP. CosustuEn

canstivcrion cat

S 1101 I (paute nant)

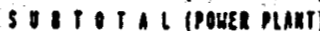

sguen plat costill

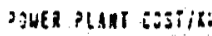

plant 1 costs

nuvi 1 s a costs

Nuit/uetifteto captrat casis hMT/UELLIEL EOST/L cuntuetLIEL cost/R

uEtt UItune costs

maxsission tint cosis

retal capitat costs

lorat 1 I cosis

DuEe nant use cost:

omeí costs:

Iotu paut hant ast cast:

UITI 24 corrinsencr:

unsea of roure platis ngEOEO

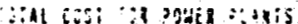
subuatine chate costs:

oveanea riansuissian costs:

Totas Pnosect captint cost: WII REPLCEIII UELIS:

12.16

3.39

1.11

1.13

1.65

23.63 aillion 1904

25.74 nillien 1598

1.47 villion 1990

4681991

2.33 sillion 1984

2.54 sillion 1994 t

38.31

15.51

I.ti

1.10

4.48

60.61 alllea 1984

63.99 abllien 1991 \&

..Al nlltion 1991 s

.11 13?1:

5.36 sillea 1834 \&

s.ll aillioa 1998

91.12 ailllea 1990 \&

1.61 sllllon 1991 :

16681938 :

91.01 cillion 1990

3.61 cilllon 1990

I85.32 aillion 1998 :

t.35 nilllen 1994 :

185.32

4.11 alllion 1990 \& itg.32 villlo" 2530

221.19

11.11

$\because$ 13 11:4: $: \div$ ji4.56 villien 1950 : 298.94 sillion 1930 ?

2965.38 silllon 1994

(125.38 silllea 1990 :

Inual mosect i a cost: 


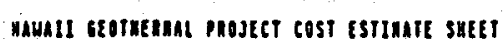
St def neshatt PGAT it?

338 des 6

IESOURCE TELPERTGR:

Nati capnetrt:

iccuacost (SF, OB, B):

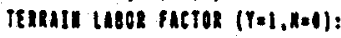

TERRII SIIE PRE $(1=1,1=1)$ :

consteuction canp ( $Y=1, n=1)$ :

Plant I I h: cou, pros., of nat

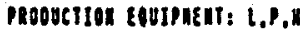

Insetton couspueat: $I, P, \|$

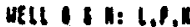

elethicity cost:

thushisstor itue olstunce:

Renintuin assessu?nt vork:

Peinits/lucenses:

1 propoction metls:

I Injection vetis:

I IEPlactuent heuls:

I Unsusetessivi utlis:

HELtFIEto captTal costs probuction EOUTPAERT

InJection EOUIPAERt

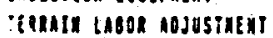

iEtruln SITE MEE. AOJUSTIEII

constavetion cane

SU:101 1.6 (neltfitio)

S 1 I I I (MELflEto) MEtLFIRI COST/M WELITIELI COST/RH

HeLI I HELHIEL I I I cosis

HEL I WELHIEL + I COSIS

PouE natr copltal cosis

coute nent

u2s iturentut

teatuin casor agjostuent

TERAII SITE MEE. hojostiEat

consinuction cinp

s I T o i l (route plati)

Sol O TA L (Poute Butit) pouze plant egsilan poute plant costicy

naxt i I l costs

puat o I cosis

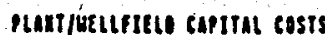

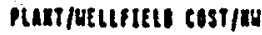
part/uetertell cast/th

weII oxlitiat costs

mitsilssion tine costs

rotat captrat costs

retut I 1 casts

PLAHT/MEL BASE CAST:

oinea costs:

rotal Mant/ueti use cost: MrTi Bu toritheEnct:

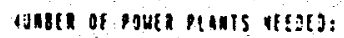

torat cost coe pouer platrs: SUtminite catle costs:

iverysad marsaisstor cists:

rotht mosect captut costs WITI RERLEenent uELIS:

irroat prosett o I I cost: ss in 14

sf

sf

:

1

1

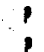

13 conts/kut

11 siles

7.51 sillion 2991

i.15 sillion last?

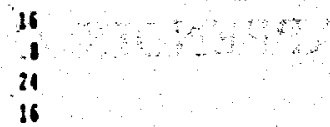

9.19

i.

i.il

1.38

1.15

19.56 aillion 1984 ,

21.30 ailliea 1990

1.39 aillion 1998

367 1990 ।

1.52 afllion iste

1.66 aillion 1954 i

39.51

11.31

1.11

1.18

1.18

\$5:12 eillion 1984 :

St.24 aillion 1991 :

a.do allion 1991 :

16951990 !

2.94 cildlon igli :

3.20 sillion 1894

12.51 alllion 1994 \&

1.19 adlllon 1490 \&

(1183 2991 i

67.31 cillion t19t 8

3.61 aillion 1998 is

is2.61 aillion 1998

t. Is alllion t991 i

152.64

J.25 cillion 1990 155.15 alllion 1998 : 202.66

$\therefore 1.11$

3126.62 allion t914 314.56 allion isi: 298.91 aillion 1918 ?

2100.12 aillion 1998 357t.62 aflllon 183 .

18.54 aillion 1996 is nawatl seotmenual prosect cost Estmate snett SO net netautf piant

nits EsTILATE--1996 otthas

iesource tempezuture:

Ptant capseItr:

recurotosy (5F, of, 1):

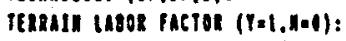

iEnuty site net $(\gamma \cdot 1, n=1)$ :

censtavetion canp $(\gamma=1, n=1)$ :

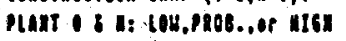

mobyetion coiptent: $1,8,4$

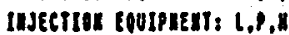

HetL । I $\mathrm{n}: \mathrm{l}, \mathrm{l}, \mathrm{H}$

etleteltity cost:

trunstisston IInE alstance: -

IenaInIne assessnent wout:

ptrnits/licensts:

I noouction uElis:

I Injection welts:

I teplucenent uELIS:

I Unsucessspt vetL:

JSI ote 6

ss an (3 anjuell)

SF

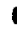

$\vdots$

1

II

.

13 coats/tut

II siles

3.11 sillion 1930

1.11 sillion 1991 :

20

11

2

\section{VELIFLL CAPITAL COSTS}

iroodetiox coutpuert

injection Eoutpagnt

itrasta labor nojusthen

tearail site paE . hosustwent

cousmoerror caup

S I T I I I (UELHELO)

SU I I I I (MELHIEL) MELPIEL COST/XI MELHEL COST/RU

Hetl S MellFitLI I I I costs UEII I MELtfieIO I I I COSIS

poue putt captTat costs

poser plint

nes maltatn?

TERHII Leor nojostaEut

retrull stfe Mes. nojostuent

consinuerion chap

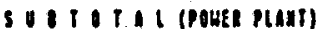

5 i i i l t (pouta plant) sutr plast azsiank poine plakt cost/zu

Dian of 1 costs

nunt is a casis

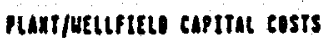
PUAT/UELIHELL COST/NH PLITT/UELLEtEL COST/RY

uEUI oxIIIIn costs

rusistisstox tunt costs

corat captrat costs

Totat o i cosis

12.16

3.39

1.11

(.13

1.65

23.13 villion 1984 \&

25.11 eillion 1998

1.47 alllion 1598.8

16) 19908

2.33 alllloa 1984

2.51 alllios 2931

39.51

18.51

1.11

1.10

1.18

61.61 eillion 1981 :

is.39 villion 1991

...1 aibia: .

ail 1991 ?

5.16 allion 1984

S.ll cillion 2896 t

91.12 silllea 1998 1.67 cilllon 4994 I65) 25918

96.11 ailllog 1998

3.60 ablllon tsse

145.32 eillitea l934

I.38 allilen Is9 1

Puer plukt use cost:

ortiet cests:

porat poute plant ust cost: HITH 318 contingencr:

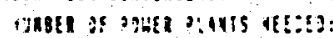

iorat cost por poute plants: suturine tade costs:

overacio inasicsion costs:

Total pesect capthat cost: utth itplacentui utlts:

185. 12

1.08 alliton 1994 11s.32 aillion 19se 211.12

i 11

:161.21 aition 2331 314.56 sillien 2991 298.94 cllldea 1990 :

3131.10 eillion 1990 1314.70 allition 1990 \&

anwol noject o i cost:

13.12 allilion 1994 

Alex Sifford is currently Geothermal Program Manager for the Resource Development Division of the Oregon Department of Energy. The following are comments from his review of the February 1988 Decision Analysts Hawaii, Inc. report on the Economic Feasibility of the Hawaii Geothermal and Cable project. 
Comments concerning the February 1988 Hawaii Department of Business and Economic Development Economic Feasibility Analysis regarding the proposed Hawaii Geothermal Project.

$\mathrm{Pg}$ P-1

(last

paragraph)

Pg ES-2

(first

paragraph)

Pg ES-2

(third

paragraph)

Pg ES-3

(mid-page

table)

Pg ES-7

(second

paragraph)

$\mathrm{Pg}$ I-2

(last

paragraph)

Pg IV-3

(first

Faragraph)

(On resource quality)

A high temperature resource is not necessarily a bigh quality resource. Hawaii has a high temperature resource but its quality is as yet unproven. Problems with seawater intrusion, silica and mineral content, unknown long-term production capability, and inherent development dangers reduce the quality potential considerably.

(On overall system planning)

To say the inclusion of Maui in the system would have no effect on its economic feasibility is unsubstantiated. Experience in the Pacific Northwest has shown interties can be difficult and expensive if not considered early in the system planning process.

(On who benefits)

Apparently all the geothermal power will to go Oahu. Does the Big Island get nothing?

(On plant sizing)

To produce 500 net $\mathrm{MW}$ of power, plants must be sized to allow for approximately $10 \%$ loss in generation and transmission.

(On development schedules)

Realistically, geothermal companies would develop the resource in stages since it can be so variade and costiy. Full and rapid development, in light of The Geysers experience, is not a logical approach.

(On plant construction time)

The report indicates it is conservative in terms of size of plants and pace of development but states its plants will be built in half the time or less than construction time estimates by Stone \& Webster Engineers of Denver, Colorado who are well experienced at design and construction.

(On steam gathering system costs) Bechtel figures for a steamgathering system is closer to $\$ 7$ million. 
Pg IV-3 (powerplant section)

$\mathrm{Pg}$ IV 4 (second paragraph)

$\mathrm{Pg}$ IV 4

(last paragraph)

Pg IV.5 (second paragraph)

Pg IV-5

(third paragraph)

Pg IV-6

(third paragraph)

Pg IV -8

(sixth paragraph)

Pg IV-9

(fourth paragraph)

Pg IV-12

(first paragraph)
(On power plant costs)

Based on Oregon Department of Energy studies, actual cost per kilowatt is for $25 \mathrm{MW}$ is between $\$ 1,600$ and $\$ 1800$. The $\$ 1.100 / \mathrm{kW}$ in the seport ( $\$ 27.734$ million divided by $25 \mathrm{MW}$ ) is very low.

(On well development)

Ten months for well development is extremely optimistic. At 13 wells per plant it allows less than one month per well. Six weeks seems more likely.

(On well O\&M costs)

The $\$ 58,000$ per well $O \& M$ cost is unsubstantiated and seems very low. Our own (Oregon Dept. of Energy) estimates show a $\$ 200,000$ to $\$ 800,000$ per well range for O\&M.

(On waste disposal costs)

The chemical and waste disposal costs are unsubstantiated.

How are these costs estimated on an undefined resource?

(On costs in general)

Where is the contingency cost for undefined resource $A$ contingency is only logical given what little is known about the resource

(On locating the plants)

Where are the maps showing voleanic activity and proposed plant locations?

(On insurance costs)

Insurance would be extraordinarily high due to risks.

The figure $0.3 \%$ seems low.

(On financing)

Twenty-four years is too long for financing. Seven years is more likely.

(On risk to investors)

Major sources of risk and uncertainty are not greatly. reduced since the development scenario is not logically staged. 
Carl Freedman is a utility economist living in Hawaii. He currently serves as Vice President of Legal Affairs and Board Member of the Blue Ocean Society. $\mathrm{He}$ is formerly a member of the Oregon Environmental Action Group Foreiaws On Board' and played an integral part in that groups examination and analysis of the proposed Pebble Springs Nuclear Facility. He has been a major participant and contributor in many comprehensive studies on conservation and alternate energy systems. The following is his assessment and critique of the February 1988 Decision Analysts Hawaii, Inc report on the economic feasibility of the proposed Hawaii Geothermal and Cable Project. 
AN ASSESSMENT AND CRITIQUE OF :

\section{DEPARTMENT OF BUSINESS AND ECONOMIC DEVELORMENT ECONOMIC FEASIBILITY ANALYSIS \\ REGARDING THE KAWAII GEOT:ERMAL/UNDERWATER CABLE PROJEニT}

PREPARED BY CARL FREEDMAN

$12 / 4 / 89$

\section{BACKGROUND}

The State of Hawail and the Hawali Electric Company (HECO) are undertaking an aggressively accelerated program to develop 500 megawatts of geothermal electrical generating capacity on the island of Hawaii in conjunction with a deep underwater and overland transmission system to transport the generated enezgy to the island of Oahu. The liawail Department of Business and Economic Development (DBED) has taken a lead role in the promotion of this enterprise.

DBED has commissioned studies which provide the basis for its conclusions that the geothermal/cable Project is economically feasible. A preliminary study was published in April of 1986: "Alternative Approaches to the Legal, Institutional and Financial Aspects of Developing an Inter-Island Electrical Transmission System.." prepared by Gerald A. Sumida et al. Subsequently, a study was commissioned to address economic concerns more specifically: "Undersea Cable to Transmit Geothermal-Generated Electrical Energy from the Island of Hawaij to Oahu: Economic Seasibility," prepared by Decision Analysts Zawai:. Zas., published in February. 1988. This latter study (DAHI study) is the basis for the projected capital costs of the geothermal/cable. Project of $\$ 1.7$ billion.

The Hawailan Electric Company (HECO) issued Requests for Proposals to private industrial consortia to solicit proposed schemes to build, finance and manage the geothermal/cable project. Four or five consortia have responded with proposals which are being reviewed by HECO and a consulting flrm. A condition in the request for proposals was that the projected delivered cost of energy to Oahu would be at or below HECo's avoided cost of energy.

According to DBED literature the project oula be sianced. built and owned by a private corporate entity (or entities, Ihe project owner would (according to DBED's interpretation) bear all of the financial risks of project cost overrins or generation and transmission problems. Revonues for the project would be provided 
by a contract with HECO, binding Heco to purchase power delivered by the project to Oahu.

\section{CONCERNS REGARDING PROJECT ECONOMICS AND FINANCING}

According to the best published hopes of DBED and HECO the geothermal/cable project could be built, financed and operated without costs or risks to ratepayers or taxpayers above what it would cost to generate electrical energy with oil-fired facilities. Ignoring all of the environmental, social. archeological, health and aesthetic issues not addressed by current economic analyses, this would be a welcome reassurance to residents of the state regarding the vulnerability of their pocketbooks.

Careful analysis of the DAHI study, however, indicates that the projected costs of building and financing the geothermal/cable =roject have been substantiaily underostimated and improper compared to HECO's avoided costs (see discussion below.) This raises concerns over the cost impacts to Hawaiian residents which are potentially enormous. The details regarding how the proposals solicited by HECO will be assessed and the particular language and terms included in any subsequent proposed contracts are of crucial importance.

(1) Will the proposals solicited by HECo indicate project costs greater than HECo's avoided costs? If so, will the project still be considered by HECO?

(2) Will the proposals solicited by HECO propose to meet avoided costs by transferring financial risks to ratepayers, iaxpayers, or utility stockholders?

(3) Will the proposals solicited by HECo incorporate lowball bids in anticipation of later re-negotiation or litigation?

Ostensibly, according to intended planned contractual arrangements, the ratepayers are to be insulated from costs exceeding HECO's avolded costs. Much previous experience with over-budget and non-functional electrical generation projects on the mainland has demonstrated that this promise may be a costly illusion. Corporations that have invested billions of dollars in a generating project in response to requests by the state of Hawaij and HECO are not going to absorb large cost overruns without liticating the matter tooth and nail in the courts. contracts irranged un the casis proposed of wod are not ijiety $=0$ be enforceable.

It is of paramount importance for these reasons to insure that any contractual agreements made by HECo, the state of Hawaii, or 
project consortia be examined very carefully to insure that they are based upon sound and reasonable economic assumptions, or they may end up being economic disasters paid for by Hawaii's ratepayers and/or taxpayers. From an economic point of view, the proposed projects will only be successful if they are in fact, actually economically prudent, regardiess of any contractual schemes or promises.

\section{ASSESSMENT OF DECISION ANALYSTS HAWAII STUDY}

\section{OVERVIEW}

Currently, DBED's economic projections of the economics of the geothermal/cable project are based upon the study by Decision Analysts Hawaii. Inc. published in February of 1988.

The study assumes that the cable and transmission system will be built and inanced by one private corporate "venture" and that the geothermal wells and generation facilities will be built by another similar venture, perhaps under the ownership of a common larger corporation. Estimates are made of the costs of building the various components of the geothermal/cable project based upon other studies and by scaling costs from other profects. The study establishes schedules of year by year expenditures, revenues, and bond sales and payments. The schedules are discounted to present values and are compared with estimates of present values of HECO's avoided fuel, operating and capital costs. By various indicators of venture profitability, break-even fuel oil cost and cost to benefit ratics, the costs of the geothermal are evaluated as being economically feasible.

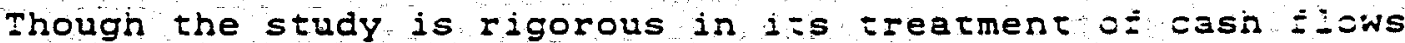
and discounting methodologies, it makes some simple errors that are of significant consequence to the outcome of its conclusions. The study assumes $100 \%$ avallability factors for geothermal generation and transmission. No transmission losses are accounted for. Assumptions are made regarding financing methodologies that are inconsistent with conventional experience and would not in certain instances be legal without legislative actions. Real generation capital cost escalation is ignored. Capital costs are in certain instances substantially underestimated. Certain methods of scaling generation plant capital costs are misapplied. 


\section{CRITIQUE}

\section{GENERATION AND TRANSMISSION AVAILABILITY}

The text of the DAHI study acknowledges that generation and transmission facilities will have some required maintenance and outage time. In the actual arithmetic of revenue calculations used in the study, however, no such adjustment is made. Revenues are calculated based upon $500 \mathrm{MW}$ of power output for 8760 hours per year (100\% availability.) The study states at one point in the text that each $25 \mathrm{MW}$ geothermal generation plant will be built to $27.5 \mathrm{MW}$ capacity to account for maintenance time, however, no such adjustment was actually made to the capital cost or operating expenses used in the calculations. The calculations used in the study assume $100 \%$ availability and $100 \%$ capacity factors for geothermal, transmission and AC-DC conversion facilities.

There is no such thing in the worid colelectical power generation as a plant operating at $100 \%$ availability. Planned maintenance and unplanned outages are inevitable. Transmission system outage percentages are typically quite small, but would be additive to generation outage times. A $90 \%$ overall avallability would be very optimistic for a geothermal/cable system. This statistic is important because it directly and proportionately effects the amount of energy delivered by the geothermal/cable system and the revenues accrued by the geothermal/cable ventures.

\section{TRANSMISSION LOSSES}

The DAHI study compares the costs of geothermal generation on the island of Hawaii to meet Oahu's needs inth iscai generatich ca Oahu. Although the study mentions in its text that revenue calculations are based upon delivered energy to Oahu (rather than generated energy) there is no accounting of transmission losses anywhere in the actual calculations of revenue or generation costs. Revenue is calculated based upon delivering $500 \mathrm{MW}$ of power to Oahu 100x of the time, generated by $500 \mathrm{MW}$ of capacity on Hawaii. Transmission losses directly and proportionately effect the amount of delivered energy and accrued revenues of the geothermal/cable venture. Transmission losses are typically at least $10 \%$ for a system like the one proposed in this project.

\section{ECONOMY OE SCALE CALCULATIONS}

The DAHI study estimates the costs of a series of twenty power plants of $25 \mathrm{MW}$ capacity. The costs for these plants are "scaled" from the documented costs of $12.5 \mathrm{MW}$ plants. The concept used in scaling is that a larger plant is cheaper fer MW because of the economy of scale. A boiler twice as big costs less than twice as 
much. The formula used in the DAFI study is the ".6 power" rule which is commonly used in scaling generation facility capital costs. According to this formula a $25 \mathrm{MW}$ plant costs $51.6 \%$ more than a 12.5 MW plant. This is an appropriate application of scaling capital costs.

The DAHI study goes further than this, however. It groups the power plants into clusters of fours and reduces the costs of the second and forth plants in each group to $70 \%$ of the scaled cost and reduces the third plant to $80 \%$ of the scaled cost. The logic used is that the plants will be close enough together that they can share certain of their facilities and thus net cost savings. The net capital costs for the network of generating facilities is reduced by this treatment of costs to an average of $80 \%$ of the previously scaled costs. This treatment is not conventional. It is especially not appropriate in this instance because of other assumptions made in the analysis. In the section of the study that addresses risks due to geological hazards it is stressed that the plants are distributed widely to avoid damage to more than one plant at a time due to lave Elows, This is a very sensitioe assumption because it is the basis for conclusions made by the study that there would be no loss in system net output and no loss in revenues due to geologic hazards (a possible loss of one plant.) Grouping the plants close enough to benefit from economies of scale is not consistent with this assumption.

Additionally, the DAHI study uses the same 6 power rule to scale the capital costs of wellfield steam-gathering equipment as it uses to scale generation plant equipment. This is inappropriate. Steam-gathering equipment does not become less expensive per MW for a larger field than for a smaller one according to a 6 power rule. If anything, much of the costs per $M W$ increase as wellfield size increases because of the longer average distances between each well and the power plant. i smalla= power plant is located in a smaller wellfield and is consequenty relatively close to the wells that supply 1t. As the size of a power plant increases, the size of the wellfield dedicated to the plant Increases and the average distance of each well to the power plant increases. Steam-gathering piping costs increase as the average distance to the power plant increases. This principle dictates that the cost per MW for steam gathering piping increases as the size of the power plant increases. The DAHI study erroneously makes the opposite assimption and calculates the cost of a $25 \mathrm{MW}$ steam-gathering system by decreasing the costs per MW of steam gathering equipment according to the .6 power rule from the documented costs for $12.5 \mathrm{MW}$ plant equipment. Fur thermore, the assumption noted above that plants will be grouped in clusters of four closely enough to benefit from economies of scale would - irthur aggravate the need ior even longer and consequentil more expensive steam-gathering equipment. 


\section{WELLFIELD COSTS}

Perhaps the most sensitive single set of assumptions regarding the costs of the geothermal/cable venture are the estimates of the costs of driling a productive wellfield. Approximately one third of the total project costs are in the welleield. The primary factor effecting wellfield costs is the number of welis necessary to develop the required thermal energy for generation purposes. some of the wells drilled would be productive. Some would be used for fluid re-injection. Some would be dry or too hot or cool. Some would need to be replaced over the life of the facility. The DAHI study assumes that 13 wells, plus eight replacement wells, will be required for each $25 \mathrm{MW}$ plant. This equates to useable/non-useable ratio of $5: 1$. This ratio may be very optimistic for Hawaij geology.

Although DBED and the DAHI study repeatediy state that geothermal resources are a proven and reliable resource, there is really very little experience in areas geologically similar to :awaii (a live volcano.) Hawail is a hot, and therefore a potentiaijy efficient resource, but it is also a very young, active and potentially unstable geological region. The area of the world with the most similar geology that has operating experience with geothermal wells is Iceland. There the experience with geothermal electrical generation has not been good. The geology seems to be too active, effecting the success rates of the wells dramatically. The project there required 24 wells to be drilled to obtain 11 that were useable. It remains to be seen how many replacement wells will be necessary. Iceland experienced problems with wells "pinching off" rendering them unusable and did not attain the sustained power levels that were anticipated. The second unit of the planned two-unit geothermal generation facility there has been abandoned because of the wellfield problems and expenses.

Without much experience with Hawailan geology, predicting the productivity and success rate of wells is quite conjectural. The wellfield success rate assumed in the DAKI study is perhaps possible, but must certainly be categorized as quite optimistic. These assumptions effect the certainty of any economic predictions dramatically.

\section{CAPITAL COST ESTIMATES}

The DAHI study is consistently optimistic about estimates of capital costs. The cost estimate for AC-DC conversion stations. for example, is $\mathrm{s} 72 \mathrm{milli}$ ion. According to current estimates from

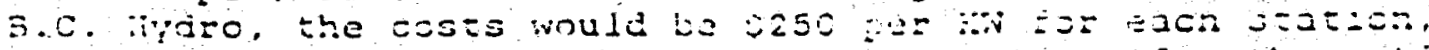
totalling $\$ 250$ million. The DAHI cost estimate for the entire transmission system including the underwater cables, overhead lines, pumping stations and AC-DC conversion station is $\$ 413.3$ million. 
The geothermal/cable project incorporates several aspects of new unproven technologies in new untried areas of geological and geographical extremes. Even in conventional projects of this magnitude it is prudent for planning purposes to include contingency costs to include what is more a proiability than a possibility of project delays, technical problems and cost overruns. No such contingencies are considered by the study.

\section{REAL COST ESCALATION}

In order to calculate HECo's future avoided costs DAHI escalates the real cost of fuel oil according to the average of a series of estimates of future oil prices. The real escalation of fue 1 prices is substantial. (Real cost escalation is the inerease over and above that due to inflation.) These avoided fuel costs are compared directly with various costs of geothermal generation. The study does not make the appropriate analogous accounting of the real jost escalation of clant capital costs. (Georhermal generation costs are primarily capital costs.l. Historical experience indicates that real plant capital costs escalate faster than real fuel prices during periods of real fuel price increases. Utility planners know, for example, that their older plants were less expensive to bulid than their newer plants, even in terms of real costs. (This may not be true in operating or fuel costs, however.) Because the DAHI study is comparative in nature, the differences in the treatment of cost escalation skew the results in favor of the geothermal/cable venture.

\section{FINANCING}

The DAHI study assumes that the cable and transmission system w11l be financed with Hawail Special Purpose Revenue Bonds (Industrial Development Bonds) at a rate $.5 \%$ above municipal bond rates. The geothermal venture is assumed to float bonds at the Aaa corporate rate. At the same tIme the study maintains that all financial risks due to cost over-runs or resource fallure are to be borne by these financing sources. These are clearly not realistic assumptions.

Hawail Special Purpose Revenue Bonds are not available for non-regulated private corporate use.

The assumption that the geothermal/cable venture can be Einanced by bonds issued at such low interest rates with such a

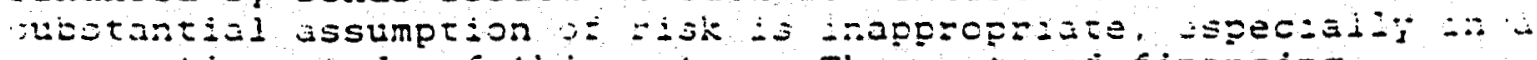
comparative study of this nature. The costs of financing appreciably effect the profitability equation used in the DAHI study. 


\section{SENSITIVITY ANALYSIS}

Throughout the DAHI study estimates and calculated numbers are associated with standard deviations to imply confidence intervals around the predicted statistics. This sort of analysis has its place in the laboratory, in demographics and perhaps around casino gambling tables. The use of confidence intervals in a study of this nature which is designed to be used by policy decisionmakers is inappropriate and misleading. To a person familiar with statistics these numbers may be of some value, but to imply to a decision-maker who may rely on the study that the values ascribed to the confidence intervals are realistic indicators of the possibility of error of the study is ludicrous.

Even from a purely statistical viewpoint the sensitivity analysis is misapplied. This type of analysis is only appropriate when all of the input parameters are truly independent of one another and are normally distributed. Neither of these conditions are met in a construction project where delays in one portion of the project can effect scheduliag and costs of other portions and where the potential for cost overruns exceed the margin of potential cost savings.

Furthermore, the sensitivity analysis only takes into account one particular type of error. It ignores the types of errors noted above, which are incremental, but when taken as a whole substantially effect the outcome of the analysis. The omission by the DAEI study of any consideration of transmission losses and availability factors effects the overall calculations in the cost comparison by at least $21 \%$, and depending on actual achieved availability factors, perhaps by $46 \%$ or more.

Approximate percentage impacts to the DAHI study groiected costs are isted beiow to give some idea of ine magnitude c: ineiz importance. These numbers are not intended as correction factors to adjust the DAHI study results to draw more accurate conclusions. They are included here to demonstrate the sensitivity of the DAEI study to its own oversights and biases. All figures are percentages of the total geothermal/cable project costs/revenues.

The cumulative percentage statistics below are only for orderof-magnitude comparison purposes. The high-end statistics may include some double-counting as, for example, in the case of generation availability being improved by additional welifield improvements . 


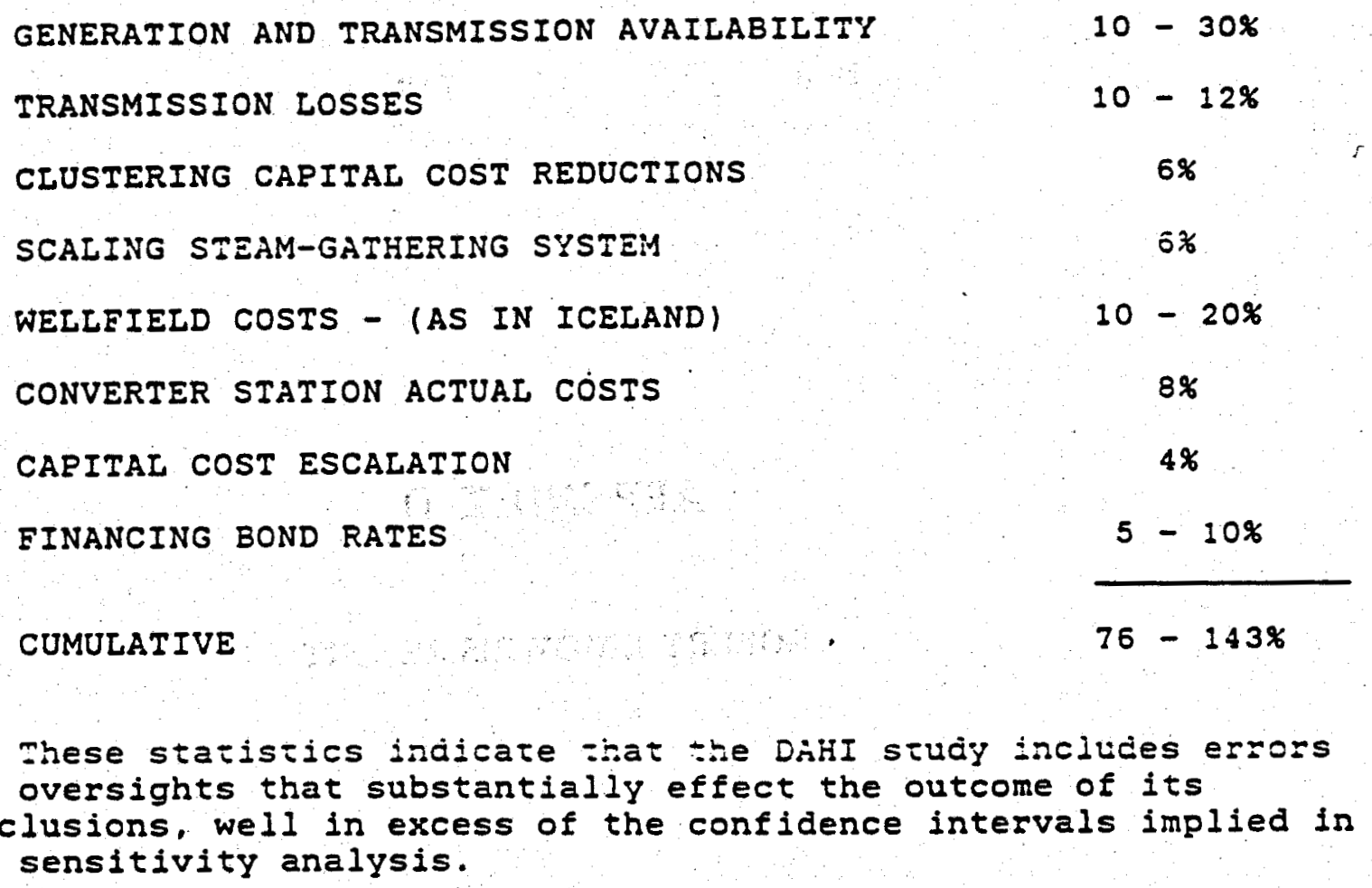

\begin{abstract}
and oversights that substantially effect the outcome of its conclusions, well in excess of the confidence intervals implied in its sensitivity analysis.
\end{abstract}

\title{
CONCLUSIONS
}

The proposed geothermal/cable project is a very large and expensive pruject with an enormous potential to impact the economy of the state of Hawail. currently existing economic analyses do not establish a sound basis for confidence in the ultimate sestefiectiveness of the project. Very carerul scruiny must je given to the details of proposed project bids and contractual arrangements to assure that financial risks and the costs of project fallures or overruns will not be assumed by Hawail's ratepayers or taxpayers or HECO's stockholders. 

APPENDIX E

COSTS IF PRODUCTION WELL INCREASES

TO 200\% REPLACEMENT 


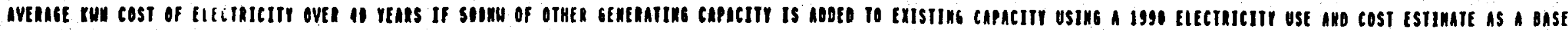
(218 Contingeney) (3/1 Noll Replacesent)

\begin{tabular}{|c|c|c|c|c|c|c|c|c|}
\hline & $\begin{array}{c}\text { EXISTIInG SYSTEL } \\
\text { (1990) }\end{array}$ & & & $\begin{array}{l}\text { EAISIIMG SYSTEN } \\
\text { (1990) }\end{array}$ & & & $\begin{array}{l}\text { Existing systen } \\
\text { (199) }\end{array}$ & \\
\hline $\begin{array}{l}11,751,211,111 \\
0,261,379,327\end{array}$ & $\begin{array}{l}\text { Total kuh caposity } \\
\text { HIth } 238 \text { Reserve }\end{array}$ & $\begin{array}{l}11,157,211,111 \\
1,261,319,321\end{array}$ & $\begin{array}{r}11,751,261,111 \\
8,261,319,321\end{array}$ & $\begin{array}{l}\text { Total iuh copacity } \\
\text { HIth 23t Reserve }\end{array}$ & $\begin{array}{r}10,751,281,111 \\
1,261,319,321\end{array}$ & $\begin{array}{r}11,151,211,011 \\
1,261,319,321\end{array}$ & $\begin{array}{l}\text { Total kWh copocity } \\
\text { HIth } 238 \text { Reserve }\end{array}$ & $\begin{array}{r}11,751,201,011 \\
1,264,319,321\end{array}$ \\
\hline $\begin{array}{r}613,911,852 \\
0,261,319,321\end{array}$ & $\begin{array}{l}\text { I Operating Revenue } \\
\text { Annual kith sold }\end{array}$ & $\begin{array}{r}613,911,852 \\
0,261,319,321\end{array}$ & $\begin{array}{r}613,974,852 \\
1,261,379,327\end{array}$ & $\begin{array}{l}1 \text { Opersating Revenue } \\
\text { Pnnual kith sold }\end{array}$ & $\begin{array}{r}613,911,852 \\
8,261,371,327\end{array}$ & $\begin{array}{r}613,314,852 \\
8,261,319,321\end{array}$ & $\begin{array}{l}2 \text { Operating Revenue } \\
\text { Innoul kuk sold }\end{array}$ & $\begin{array}{r}613,911,852 \\
8,261,319,321\end{array}$ \\
\hline 1.13 & Avoregi conts/kuh & 1.13 & 1.13 & Average conts/kuh & 1.43 & 1.13 & Averoge Cents/kth & 1.13 \\
\hline $\begin{array}{l}\text { cEotmennal } \\
\text { Lou }\end{array}$ & $\begin{array}{l}\text { ROOE O CAPACIIY } \\
\text { (25nh Plants) } \\
\text { (11 Yoers) }\end{array}$ & $\begin{array}{l}\text { Geormennat } \\
\text { ntGh }\end{array}$ & $\begin{array}{l}\text { GEOHEERII } \\
\text { LOW }\end{array}$ & 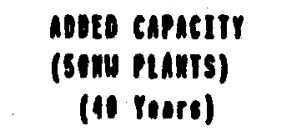 & $\begin{array}{l}\text { GeOTHE } \\
\text { HIGH }\end{array}$ & SoLAR/OIt & 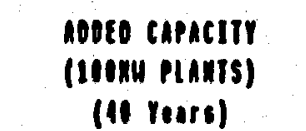 & oIt \\
\hline $\begin{array}{r}13,211,111,111 \\
111,791,111,111\end{array}$ & $\begin{array}{l}\text { I Projeet cost } \\
\text { twh sold }\end{array}$ & $\begin{array}{r}16,561,011,111 \\
111,191,011,011\end{array}$ & $\begin{array}{l}12,131,111,111 \\
91,813,111,111\end{array}$ & $\begin{array}{l}1 \text { Projoet cost } \\
\text { num sold }\end{array}$ & $\begin{array}{l}15,111,111,111 \\
98,113,111,011\end{array}$ & $\begin{array}{l}11,610,1110,110 \\
98,112,100,010\end{array}$ & $\begin{array}{l}1 \text { Project cost } \\
\text { kun sold }\end{array}$ & $\begin{array}{l}8,511,0101,110 \\
98,112,110,011\end{array}$ \\
\hline 13.11 & $\begin{array}{l}\text { Average conte/kuh } \\
\text { Ulth Royalty }\end{array}$ & 16.21 & 12.11 & $\begin{array}{l}\text { Average Conts/kWh } \\
\text { WIth Royalty }\end{array}$ & 15.19 & 10.11 & $\begin{array}{l}\text { Average Cents/kuh } \\
\text { uIth Royolty }\end{array}$ & 1.13 \\
\hline $31,221,991,011$ & $\begin{array}{l}\text { Consinic capacity } \\
\text { (11 reers) } \\
\text { opersting Revenue }\end{array}$ & 11,118 & 991,011 & 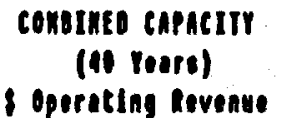 & . 171 & 35,16 & $\begin{array}{c}\text { conotued capacitr } \\
\text { (10 rours) } \\
\text { operatlag levenue }\end{array}$ & 14,011 \\
\hline $411,1111,145,316$ & kwn sold & $101,111,015,316$ & $116,119,169,119$ & tuhn sold & $106,109,169,1199$ & $105,951,019,119$ & nuh suld & $105,951,619,119$ \\
\hline 9.25 & Iverags cents/kuh & 18.16 & 9.11 & Average cente/kuh & 9.13 & 8.66 & Avorage Cents/kuh & 8.16 \\
\hline 1.83 & $\begin{array}{l}\text { Conts/huin Incroust } \\
\text { ulth nosod capaclty }\end{array}$ & 2.63 & 1.51 & $\begin{array}{l}\text { Cente/thh Inerouse } \\
\text { Wt th idded copacity }\end{array}$ & 2.31 & 1.23 & $\begin{array}{l}\text { Conts/huh Increase } \\
\text { With Rdod Capecity }\end{array}$ & 0.13 \\
\hline 29.68 & in added to & 35.11 & 21.28 & $\begin{array}{l}\text { Cente/kuh I Inerease } \\
\text { uith noded copeclty }\end{array}$ & 1.11 & 16.68 & $\begin{array}{l}\text { Conts/kuh I Increase } \\
\text { uith nded copocity }\end{array}$ & 9.11 \\
\hline
\end{tabular}




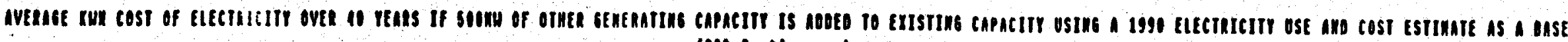
(3it Contingeney)

(J/1 Well Replocesent)

\begin{tabular}{|c|c|c|c|c|c|c|c|c|}
\hline & $\begin{array}{l}\text { ExIstini sisten } \\
\text { (19si) }\end{array}$ & & \multicolumn{3}{|c|}{$\begin{array}{l}\text { ExIstint systen } \\
\text { (1990) }\end{array}$} & \multicolumn{3}{|c|}{$\begin{array}{c}\text { ExIstIII systen } \\
\text { (1995) }\end{array}$} \\
\hline $\begin{array}{l}10,131,281,111 \\
1,261,319,321\end{array}$ & $\begin{array}{l}\text { Total wh copactey } \\
\text { Uith } 231 \text { Reserve }\end{array}$ & $\begin{array}{r}11,151,211,111 \\
8,261,379,321\end{array}$ & $\begin{array}{l}11,151,201,011 \\
1,261,319,321\end{array}$ & $\begin{array}{l}\text { Total the copoeity } \\
\text { uIth ezs Reserve }\end{array}$ & $\begin{array}{r}11,151,211,111 \\
1,261,319,321\end{array}$ & $\begin{array}{r}10,751,2210,111 \\
8,264,379,321\end{array}$ & $\begin{array}{l}\text { Total kh Copocity } \\
\text { UIth } 238 \text { Remerve }\end{array}$ & $\begin{array}{r}11,151,201,111 \\
8,261,319,321\end{array}$ \\
\hline $\begin{array}{r}613,911,052 \\
1,261,319,321\end{array}$ & $\begin{array}{l}\text { I peeriting hevenue } \\
\text { inawel thil sold }\end{array}$ & $\begin{array}{r}613,971,052 \\
1,261,379,321\end{array}$ & $\begin{array}{l}611,911,052 \\
1,201,319,321\end{array}$ & $\begin{array}{l}\text { S Operatling Rovenue } \\
\text { Annuel tent sold }\end{array}$ & $\begin{array}{r}613,911,052 \\
1,261,319,321\end{array}$ & $\begin{array}{r}613,971,852 \\
0,262,379,321\end{array}$ & $\begin{array}{l}\text { I Operating Revenue: } \\
\text { Annual tith sold }\end{array}$ & $\begin{array}{r}613,914,852 \\
8,261,379,321\end{array}$ \\
\hline 1.13 & Average Conle/kuh & 1.13 & 1.13 & Average Cents/tth & 1.13 & 1.13 & Average Cents/luph & 1.13 \\
\hline $\begin{array}{c}\text { IEOTMEA1111 } \\
\text { 1011 } \\
13,911,011,011 \\
111,131,011,111\end{array}$ & 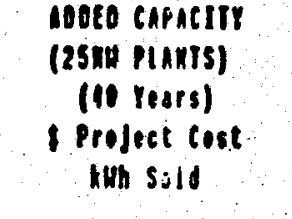 & 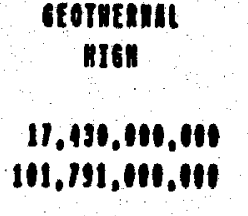 & $\begin{array}{c}\text { GEOTHEAMAL } \\
\text { IOH } \\
12,651,111,1110 \\
\$ 1,011,111,111\end{array}$ & 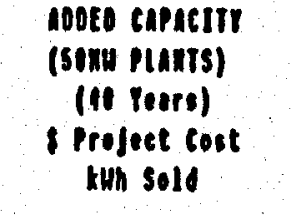 & $\begin{array}{c}\text { SEOTMELnNI } \\
\text { NIGn } \\
15,111,111,111 \\
98,013,111,111\end{array}$ & $\begin{array}{l}10,611,111,011 \\
98,112,111,110\end{array}$ & 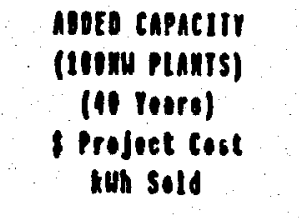 & $\begin{array}{r}1,511,011,011 \\
98,112,011,0111\end{array}$ \\
\hline 13.12 & $\begin{array}{l}\text { Average Cor.ts/twh } \\
\text { wien loysity }\end{array}$ & 17.12 & 12.11 & $\begin{array}{l}\text { Average Cents/kuh } \\
\text { uIth Ropolty }\end{array}$ & 15.91 & 16.81 & $\begin{array}{l}\text { Average Cents/kuh } \\
\text { WIth lopelty }\end{array}$ & 0.13 \\
\hline $31,521,994,011$ & 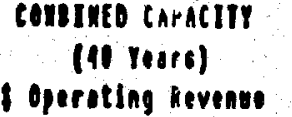 & $, 988,994,011$ & $31,211,991,0114$ & 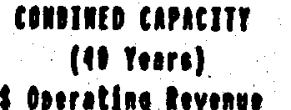 & & & $\begin{array}{c}\text { Consmed capacity } \\
\text { (11) rears) }\end{array}$ & ${ }^{3}$ \\
\hline $6.111,115,306$ & tuh s.ld & $911,111,115,316$ & $116,469,169,119$ & $\therefore$ kns sold & $(116,189,169,119$ & (15, S51,019,119 & knh sold & $115,951,619,109$ \\
\hline 9.13 & Averoge cencs/kth & 10.21 & 9.15 & Average Conte/kth & 9.92 & 0.66 & Avorage Cents/kth & 0.16 \\
\hline 2.11 & $\begin{array}{l}\text { Conts/kwh lincroese } \\
\text { uIth ndded capaclty }\end{array}$ & 2.81 & 1.12 & $\begin{array}{l}\text { Conts/kuh Increase } \\
\text { uIth noded capoeity }\end{array}$ & 2.19 & 1.23 & $\begin{array}{l}\text { Conts/kuh Incresse } \\
\text { Hith ndded tapocity }\end{array}$ & 0.13 \\
\hline 26.98 & $\begin{array}{l}\text { Cente/tuth I increase } \\
\text { ulth ides capaelty }\end{array}$ & 38.38 & 23.28 & $\begin{array}{l}\text { Cents/tuh I Inerease } \\
\text { Wlth ndded Capaclty }\end{array}$ & 33.61 & 16.68 & $\begin{array}{l}\text { Cente/kuth I Incroase } \\
\text { WIth ndded Capeclty }\end{array}$ & 9.88 \\
\hline
\end{tabular}




\section{AN ASSESSMENT AND CRITIQUE OF:}

\section{DEPARTMENT OF BUSINESS AND ECONOMIC DEVELOI'MENT ECONOMIC FEASIBILITY ANALYSIS REGARDING THE HAWAII GEOTHERMAL/UNDERWATER CABLE PROJECT}

PREPARED BY CARL FREEDMAN

$12 / 4 / 89$

\section{BACKGROUND}

The State of Hawall and the Hawall Electric Company (HECO) are undertaking an aggressively accelerated program to develop 500 megawatts of geothermal electrical generating capacity on the island of Hawall in conjunction with a deep underwater and overland transmisston system to transport the generated energy to the island of Oahu. The Hawall Department of Business and Economic Development (DBED) has taken a lead role in the promotion of this enterprise.

DBED has commissioned studies which provide the basis for its conclusions that the geothermal/cable Project is economically feasible. A prellminary study was publlshed in April of 1986: "Alternative Approaches to the Legal, Instltutional and Financial Aspects of Doveloping an Inter-Island Electrical Transmission System.." prepared by Gerald A. Sumlda et al. Subsequently, a study was commlssioned to address economic concerns more spectfically: "Undersea Cable to Transmit Geothermal-Generated Electrlcal Energy from the Island of Hawall to Oahu: Economic Feasibility," prepared by Decislon Analysts Hawail, Inc., published in February. 1988. This latter study (DAHI study) is the basis for the projected capltal costs of the geothermal/cable Project of $\$ 1.7$ billion.

The Hawallan Eloctric Company (HECO) Issued Requests for Proposals to private Industrial consortia to soliclt proposed schemes to build, finance and manage the geothermal/cable Project. Four or five consortla have responded with proposals which are being revlewed by HECO and a consulting firm. A condition in the request for proposals was that the projected delivered cost of energy to Oahiu would be at or below HECO's avolded cost of energy. 
According to DBED literature the project would be financed, bult and owned by a private corporate entity (or entities.) The project owner would (according to DBED's Interpretation) bear all of the financlal risks of project cost overruns or generation and transmission problems. Revenues for the project would be provided by a contract with HECO, binding Heco to purchase power dellvered by the project to Oahu.

\section{CONCLRNS REGARUING UROIECL RCONOMICSANU NINANCING}

According to the best published hopes of DBED and HECO the geothermal/cable project could be built, financed and operated without costs or risks to ratepayers or taxpayers above what it would cost to generate electrlcal energy with oif-fired facilities. Ignoring all of the environmental, social, archeological, health and aesthetic issues not addressed by current economic analyses, this would be a welcome reassurance to residents of the state regarding the vulnerability of their pocketbooks.

Careful analysls of the DAHI study, however, Indicates that the projected costs of bullding and financing the geothermal/cable project have been substantially underestimated and improperly compared to HECO's avolded costs (see discussion below.) This ralses concerns over the cost impacts to Hawallan residents which are potentlally enormous. The detalls regarding how the proposals solictted by HECO will be assessed and the particular language and terms included in any subsequent proposed contracts are of cruclal importance.

(1) Will the proposals solicited by HECO Indicate project costs greater than HECO's avolded costs? if so, will the project still be considered by HECO?

(2) Wil the proposals solicited by HECO propose to meet avoided costs by transferring financial risks to ratepayers, taxpayers, or utility stockholders ?

(3) Will the proposals sollctted by HECO Incorporate low-ball bids in anticipation of later re-negotiation or ittigation?

Ostensibly, according to intended planned contractual arrangements, the ratepayers are to be Insulated from costs exceeding HECO's avolded costs. Much previous experlence with over-budget and non-functional electrical generation projects on the mainland has demonstrated that this promise may be a costly ilfusion. Corporations that have invested billions of dollars in a generating project in response 
to requests by the State of Hawall and HECO are not golng to absorb large cost overruns without litlgating the matter tooth and nall in the courts. Contracts arranged on the basis proposed by DBED are not likely to be enforceable.

It is of paramount importance for these reasons to Insure that any contractual agreements made by HECO, the State of Hawall, or project consortla be examined very carefully to Insure that they are based upon sound and reasonable economlc assumptions, or they may end up belng economic dlsasters pald for by Hawall's ratepayers and/or taxpayers. From an economic point of view, the proposed projects will only be successful if they are in fact, actually economically prudent, regardless of any contractual schemes or promises.

\section{ASSESSMENT OE DECISION ANALYSTS HAWAII STUDY}

\section{OVERVIEW}

Currently, DBED's economic projections of the economics of the geothermal/cable project are based upon the study by Declsion Analysts Hawall, Inc. published in February of 1988.

The study assumes that the cable and transmission system will bo bullt and Inanced by one private corporate "venture" and that the geothermal wells and generatlon facilities will be built by another similar venture. perhaps under the ownership of a common larger corporatlon. Estimates are made of the costs of bullding the various components of the geothermal/cable project based upon other studies and by scaling costs from other projects. The study establishes schedules of year by year expenditures, revenues, and bond sales and payments. The schedules are discounted to present values and are compared with estimates of present values of HECO's avolded fuel, operating and capltal costs. By various Indlcators of venture profitabllity, break-even fuel oll cost and cost to benefit ratlos, the costs of the geothermal are evaluated as being economically feasible.

Though the study is igorous in tis treatment of cash flows and discounting methodologies, it makes some simple errors that are of signilicant consequence to the outcome of its conclusions. The study assumes 100\% availability factors for geothermal generation and transmission. No transmission losses are accounted for. Assumptions are made regarding financing methodologies that are inconsistent with conventlonal experlence and would not In certain Instances be legal wilhout legislatlve actions. Real generatlon capital cost escalation is Ignored. Capltal costs are in certain 
Instances substantially underestimated. Certaln methods of scaling generation plant capltal costs are misapplied.

\section{GENERATION AND TRANSMISSION AVAILABIIIIIY}

The text of the DAHI study acknowledges that generation and transmission facilities will have some required maintenance and outage time. In the actual arithmetic of revenue calculations used in the study, however, no such adjustment is made. Revenues are calculated based upon $500 \mathrm{MW}$ of power output for 8760 hours per year (100\% avallabillty.) The study states at one point in the text that each $25 \mathrm{MW}$ geothermal generation plant will be built to $27.5 \mathrm{MW}$ capacity to account for maintenance time, however, no such adjustment was actually made to the capltal cost or operating expenses used in the calculations. The calculations used in the study assume $100 \%$ avallability and $100 \%$ capacity factors for geothermal, transmission and AC-DC conversion facilities.

There is no such thing in the world of electrical power generation as a plant operating at $100 \%$ avallability. Planned maintenance and unplanned outages are Inevitable. Transmission system outage percentages are typically quite small, but would be additive to generation outage times. A $90 \%$ overall avallabillty would be very optimistic for a geothermal/cable system. This statistic is important because it directly and proportionately effects the amount of energy delivered by the geothermal/cable system and the revenues accrued by the geothermal/cable ventures.

\section{TRANSMISSION LOSSES}

The DAHI study compares the costs of geothermal generation on the island of Hawail to meet Oahu's needs with local generation on Oahu. Although the study mentions in its text that revenue calculations are based upon dellvored energy to Oahu (rather than generated energy) there is no accounting of transmission losses . anywhere in the actual calculations of revenue or generation costs. Revenue is calculated based upon dellvering $500 \mathrm{MW}$ of power to Oahu $100 \%$ of the time, generated by $500 \mathrm{MW}$ of capacity on Hawall. Transmission losses directly and proportionately effect the amount of delivered energy and accrued revenues of the geothermal/cable venture. Transmission losses are typically at least $10 \%$ for a system like the one proposed in this project. 


\section{ECONOMY OF SCALE CALCULA'TIONS}

The DAHI study estimates the costs of a serles of twenty power plants of 25 MW capacity. The costs for these plants are "scaled" from the documented costs of 12.5 MW plants. The concept used in scaling is that a larger plant is cheaper per MW because of the economy of scale. A boller twice as blg costs less than twice as much. The formula used in the DAHI study is the ".6 power" rule which is commonly used in scaling generation facility capltal costs. According to thls formula a $25 \mathrm{MW}$ plant costs $51.6 \%$ more than a $12.5 \mathrm{MW}$ plant. This is an approprlate application of scaling capltal costs.

The DAHI study goes further than this, however. It groups the power plants Into clusters of fours and reduces the costs of the second and forth plants in each group to $70 \%$ of the scaled cost and reduces the third plant to $80 \%$ of the scaled cost. The logic used is that the plants will be close enough together that they can share certain of their facillties and thus net cost savings. The net capltal costs for the network of generating facillties is reduced by this treatment of costs to an average of $80 \%$ of the previously scaled costs. This treatment is not conventional. It is especlally not appropriate in this instance because of other assumptions made in the analysis. In the section of the study that addresses risks due to geological hazards it is stressed that the plants are distributed widely to avold damage to more than one plant at a time due to lave flows. This is a very sensitive assumption because it is the basis for conclusions made by the study that there would be no loss in system net output and no loss in revenues due to geologic hazards (a possible loss of one plant.) Grouping the plants close enough to beneflt from economles of scale is not consistent with this assumption.

Addltionally, the DAHI study uses the same .6 power rule to scale the capital costs of wellfield stearn-gathering equipment as it uses to scale generation plant equipment. This is inapproprlate. Steam-gathering equipment does not become less expensive per MW for a larger field than for a smaller one according to a .6 power rule. If anything, much of the costs per MW Increase as wellfield size increases because of the longer average distances between each well and the power plant. A smaller power plant is located in a smaller wellfield and is consequently relatively close to the wells that supply It. As the size of a power plant Increases, the size of the wellifeld dedicated to the plant increases and the average distance of each well to the power plant increases. Steam-gathering plping costs increase as the average distance to the power plant increases. This principle dictates that the cost per MW for steam galtering piplng increases as the size of the power plant increases. The DAIII study erroneously makes the opposite assumption and calculatos the cost of a $25 \mathrm{MW}$ steam-gathering system by decreasing the costs per MW of steam gathering equipment according to the .6 power rule from the documented costs for $12.5 \mathrm{MW}$ plant equipment. Furthermore, the assumption noted above that plants will be grouped in clusters of four closely enough to benelit from economies of scale would further 
aggravate the need for even longer and consequently more expensive steam-gathering equipment.

\section{WELLFIELD COSTS}

Perhaps the most sensitive single set of assumptions regarding the costs of the geothermal/cable venture are the estimates of the costs of drilling a productive wellfield. Approximately one third of the total project costs are in the wellfield. The primary factor effecting wellfield costs is the number of wells necessary to develop the required thermal energy for generation purposes. Some of the wells drilled would be productive. Some would be used for fluid re-Injection. Some would be dry, or too hot or cool. Some would need to be replaced over the life of the facility. The DAHI study assumes that 13 wells, plus eight replacement wells, will be required for each 25 MW plant This equates to useable/non-useable ratlo of 5:1. Thls ratio may be very optimistic for Hawall geology.

Although DBED and the DAHI study repeatedly state that geothermal resources are a proven and reliable resource, there is really very little experlence in areas geologically similar to Hawall (a live volcano.) Fawall is a hot, and therefore a potentlally efficient resource, but it is also a very young, active and potentlally unstable geological region. The area of the world with the most similar geology that has operating experlence with geothermal wells is lceland. There the experience with geothermal electrical generation has not been good. The geology seems to be too active, effecting the success rates of the wells dramatically. The project there required 24 wells to be drilled to obtain 11 that were useable. It remains to be seen how many replacement wells will be necessary. Iceland experlenced problems with wells "pinching off" rendering them unusable and did not attain the sustained power levels that were anticipated. The second unit of the planned two-unit geothermal generation facility there has been abandoned because of the wellileld problems and expenses.

Without much experience with Hawallan geology, predicting the productivity and success rate of wells is quite conjectural. The wellfield success rate assumed in the DAHI study is perhaps possible, but must certainly be categorized as quite optimistic. These assumptions effect the certainty of any economic predictions dramatically.

\section{CAPITAL COST ESTIMATES}

The DAHI study is consistently optimistlc about estimates of capltal costs. The cost estimate for AC-DC conversion stations, for example, is $\$ 72$ million. According to current estimates from B.C. Hydro, the costs would be $\$ 250$ per $\mathrm{KW}$ for each station, totalling $\$ 250$ million. The DAHI cost estimate for the entire transmission 
system including the underwater cables, overhead lines, pumping stations and AC-DC conversion station is $\$ \mathbf{4 1 3 . 3}$ million.

The geothermal/cable project incorporates several aspects of new unproven technologies in new untried areas of geological and geographical extremes. Even in conventional projects of this magnitude it is prudent for planning purposes to include contingency costs to Include what is more a probability than a possiblity of project delays, technical problems and cost overruns. No such contingencles are considered by the study.

\section{REAL COST ESCALATION}

In order to calculate HECO's future avolded costs DAHI escalates the real cost of fuel oll according to the average of a serles of estimates of future oll prices. The real escalation of fuel prices is substantial. (Real cost escalation is the increase over and above that due to Inflation.) These avolded fuel costs are compared directly with various costs of geothermal generation. The study does not make the approprlate analogous accounting of the real cost escalation of plant capital costs. (Geothermal generation costs are primarily capital costs.) Historkal experience indicates that real plant capltal costs escalate faster than real fuel prices during periods of real fuel price Increases. Utility planners know, for example, that their older plants were less expensive to bulld than their newer plants, even in terms of real costs. (This may not be true In operating or fuel costs, however.) Because the DAHI study is comparative in nature, the differences in the treatment of cost escalation skew the results in favor of the geothermal/cable venture.

\section{FINANCING}

The DAHI study assumes that the cable and transmission system will be financed with Hawall Special Purpose Revenue Bonds (Industrial Development Bonds) at a rate $.5 \%$ above municipal bond rates. The geothermal venture is assumed to float bonds at $.5 \%$ above the Aaa corporate rate. At the same time the study malntains that all financlal rlsks due to cost over-runs or resource fallure are to be borne by these financing sources. These are clearly not realistic assumptions.

The issuance of Hawall Special Purpose Revenue Bonds for the cable project would require special action by the Hawall State Legislature.

The assumption that the geothermal/cable venture can be financed by bonds Issued at such low interest rates with such a substantlal assumption of risk is 
inappropriate, especially in a comparatlve study of this nature. The costs of financing appreclably effect the profitability equation used in the DAHI study.

\section{SENSITIVITY ANALYSIS}

Throughout the DAHI study estimates and calculated numbers are assoclated with standard deviations to imply confidence intervals around the predicted statistics. This sort of analysis has its place in the laboratory. in demographics and perhaps around casino gambling tables. The use of confidence intervals in a study of this nature which is designed to be used by policy decision-makers is inappropriate and misleading. To a person familliar with statistics these numbers may be of some value, but to imply to a decislon-maker who may rely on the study that the values ascribed to the confidence Intervals are realistic Indicators of the possibility of error of the study is ludicrous.

Even from a purely statistical vlewpoint the sensittivity analysis is misapplled. This type of analysis is only appropriate when all of the input parameters are truly Independent of one another and are normally distrlbuted. Nelther of these conditions are met in a construction project where delays in one portion of the project can effect scheduling and costs of other portions and where the potential for cost overruns exceed the margin of potential cost savings.

Furthermore, the sensitivity analysis only takes into account one particular type of error. It Ignores the types of errors noted above, which are incremental, but when taken as a whole substantially effect the outcome of the analysis. The omission by the DAHI study of any consideration of transmission losses and avallabllity factors effects the overall calculations in the cost comparison by at least $21 \%$, and depending on actual achleved avallablilty factors, perhaps by $46 \%$ or more.

Approximate percentage Impacts to the DAHI study projected costs are listed below to give some Idea of the magnitude of their Importance. These numbers are not intended as correction factors to adjust the DAHI study results to draw more accurate conclusions. They are Included here to demonstrate the sensitivity of the DAHI study to its own oversights and blases. All figures are percentages of the total geothermal/cable project costs/revenues.

The cuinulative percentage statistics below are only for order-of-magnitude comparison purposes. The high-end statlstics may Include some double-counting as, for example, in the case of generation avallability being improved by additional wellifeld improvements. 


\begin{tabular}{lc} 
GENERATION/TRANSMISSION AVAILABILITY & $10 \cdot 30 \%$ \\
TRANSMISSION LOSSES & $10 \cdot 12 \%$ \\
CLUSTERING CAPITAL COST REDUCTIONS & $6 \%$ \\
SCALING STEAM-GATHERING SYSTEM & $6 \%$ \\
WELLFIELD COSTS - (AS IN ICELAND) & $10-20 \%$ \\
CONVERTER STATION ACTUAL COSTS & $8 \%$ \\
CAPITAL COST ESCALATION & $4 \%$ \\
FINANCING BOND RATES & $5-10 \%$ \\
\hline \\
CUMULATIVE
\end{tabular}

These statlstics Indicate that the DAHl study includes errors and oversights that substantlally effect the outcome of its conclusions, well in excess of the confldence Intervals implied in its sensittvity analysis.

\section{CONCLUSIONS}

The proposed geothermal/cable prolect is a very large and expensive project with an enormous potential to Impact the economy of the State of Hawall. Currently existing economic analyses do not establish a sound basis for confidence in the ultimate costeffectiveness of the project. Very careful scrutiny must be given to the detalls of proposed project bids and contractual arrangements to assure that financlal risks and the costs of project fallures or overruns will not be assumed by Hawall's ratepayers or taxpayers or HECO's stockholders. 


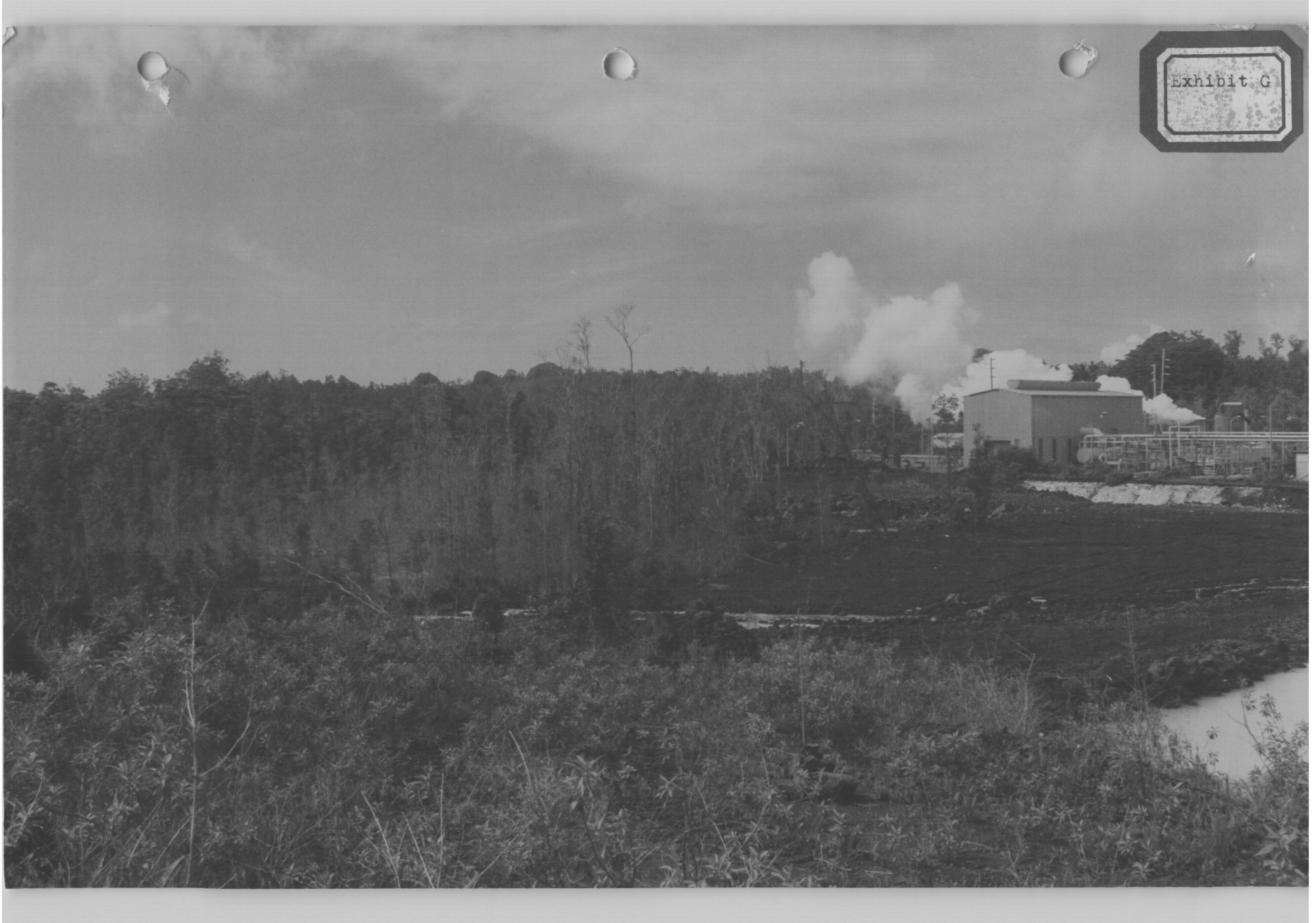


8

\section{a}

c
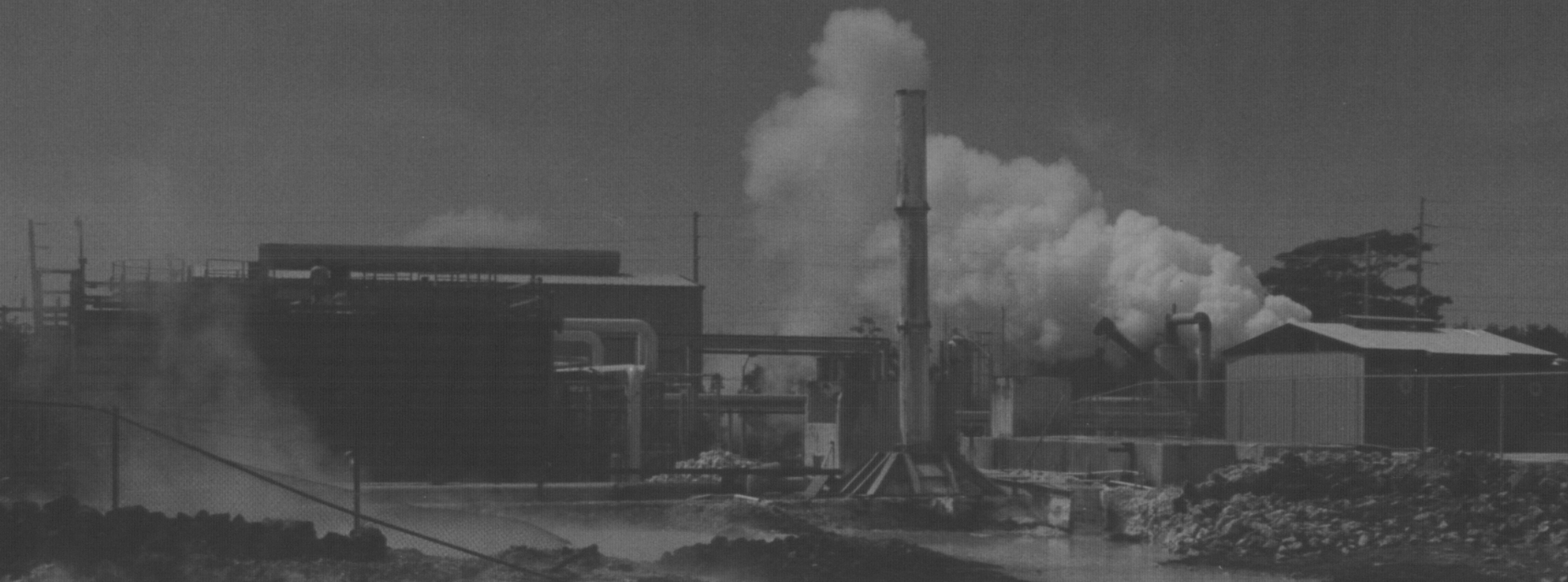


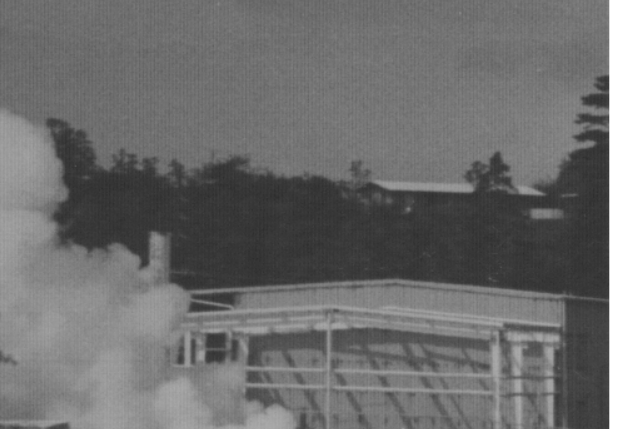
$+2 .+4 x^{2}$ 3
$x^{2}=$ 


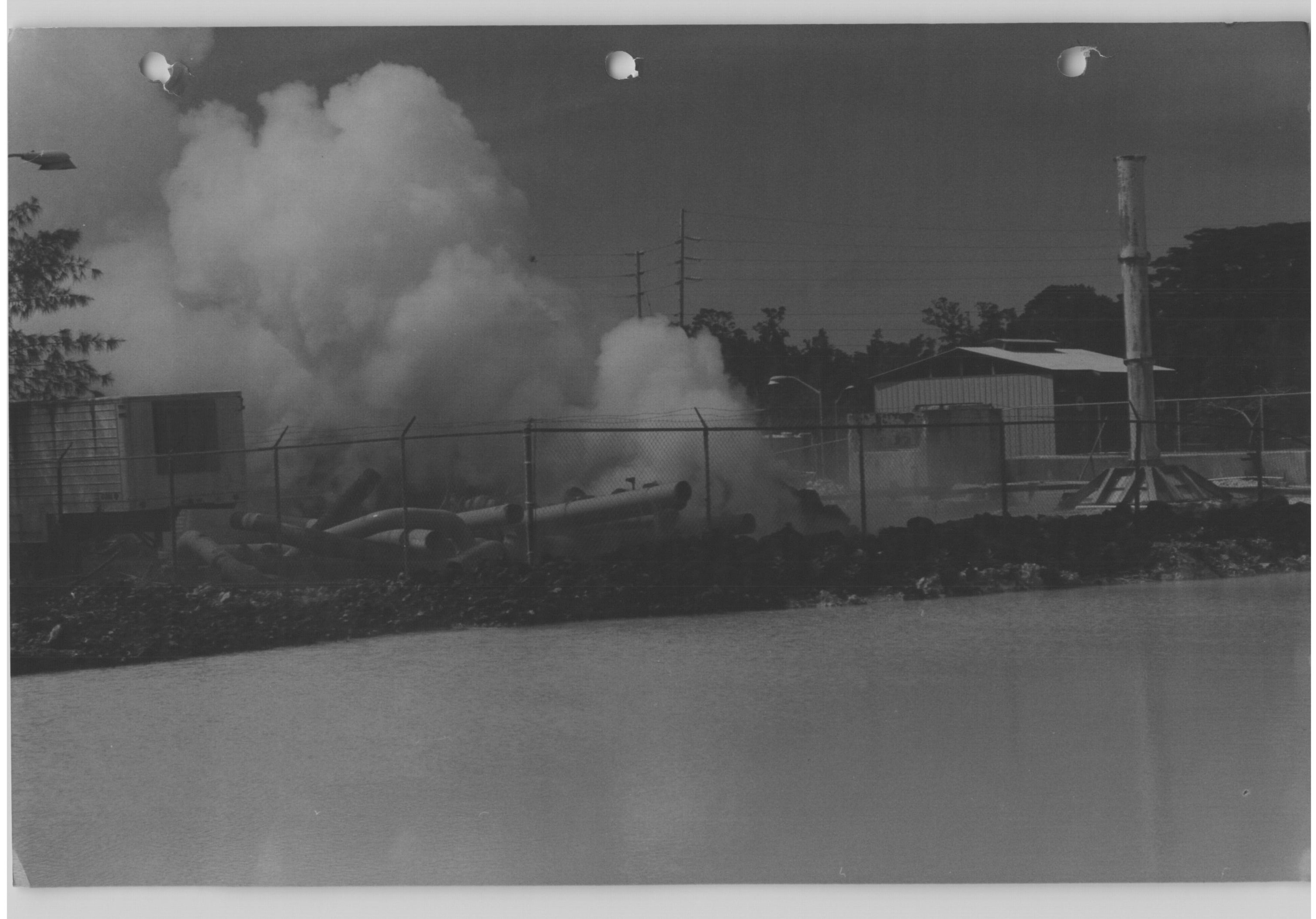




\title{
GEOTHERMAL RESOURCES
}

OF THE

KILAUEA EAST RIFT ZONE
Prepared from Public Records
by

\author{
William L. D'Olier
}

Geothermal Industry Consultant

California Registered Geologist, No. 1883 
Neither hawaiian Electric Company, Stone \& Nebster Engineezing Corporation nor any of the contributors to this document mates an: warranty or representation (expressed or implied) with respeat the accuracy, completeness, or usefulness of the information contained in this document. Hawaiian Electric Company and Stone $\varepsilon$ Webster Engineering Corporation assume no responsibility for liability or damage which may result from the use of any of the information contained in this document. 


\section{APPENDIX A}

GEOTHERMAL RESOURCES OF THE KILAUEA EAST RIFT ZONE

\section{A.1 HAWAIIAN ISLANDS - ORIGIN AND ACTIVITY}

The island of Hawaii is the newest member of a chain of volcanoes that have repeatedly matured as major islands in the middle of the northern Pacific Ocean. An obscure complex of processes is generating inordinate quantities of magma in a deep earti phenomena, the mantle plume or mantle hot spot. Within the plume, at depths of 60 kilometers and more, the Hawaiian basaltic magma (tholeite) forms at temperatures of 1350 to $1400^{\circ} \mathrm{C}$. These high temperatures impart an extreme Eluidity and density reduction to the magma. The upward mass movement of magma easily penetrates the relatively thin oceanic crustal plate and rapidly constructs new volcanoes on the deep ocean floor (Decker, 1987).

The Hawailan mantle hot spot, fixed in position and operating as an energy and mass transfer system for more than 70 milion years, is undeterred by the steady northwestward movement of the pacific crustal plate above it. This plate movement has preserved a trail of older volcanoes and seamounts, The Hawaiian-Emperor Volcanic Chain, which courses straight and west-northwest for 3550 kilometers. After a $60^{\circ}$ right bend, the chain holds a straight, north-northwest course for an additional 2600 kilometers before its destruction, with the Pacific crustal plate, by subduction in the Aleutian Trench. The 3550 kilometer distance betneen currently active volcanic centers (southeastern island of Hawaii) and the bend represents 44 million years (my) of relatively continuous and increasing magma production by the Hawailan hot spot. The volcanic rock produced, an approximate volume of 750,000 cubic kilometers, now stands on the seafloor as the long, linear Hawaiian Ridge. The potassium-argon age dates of lava 
rocks in the state of Hawail range from 5.7 to $5.4 \mathrm{my}$ : respectively, on Nihau and Kauai, to 0.375 - 0.4 my at Mauna zez and Mauna Loa, the giant shield volcanoes on Hawail. volcarid growth studies indicate that the Hawaiian hot spot is present?y generating lava volumes at the greatest eruptive rates in its known history (Clague \& Dalrymple, 1987).

The island of Hawaii is one of the largest volcanic mountains on the earth. It is a composite structure of five volcanic centers including the two mighty shield volcanoes Mauna Kea and Mauna Loa. often snow covered, these two young peaks stand nearly 4200 meters above sea level and nearly 9700 meters above the ocean floor in the Hawaiian Trough, a submarine basin northeast of the island. The island's land area of 10,438 square kilometers has maximum dimensions of 150 kilometers $\mathrm{N}-\mathrm{S}$ and 129 kilometers $\mathrm{W}-\mathrm{E}$. Oniy 11 percent of the total volcanic rock mass rises above sea level. Initial lava eruptions on the ocean floor constructed volcanic seamounts, probably first broaching sequentially as separate islands, then rapidiy coalescing to form the large, young, present island of Hawaii.

The five volcanic centers on the island of Hawaii, in sequence of diminishing age, are Kohala, Mauna Kea, Eualalai, Mauna Loa and kilauea. The southeastward trends of increasing youth, voicanic activity and seismicity are even more evident with the inclusion of the active volcanic seamount, Loini, 50 kilometers south of Kilauea's summit caldera with its summit 970 meters below sea level (see Figure A-1 and Malahoff, 1987). Table A-1 presents key information on the ages and sequence of volcanic activity at these six centers.

The magma and lava processes, now operating in their upper dynamic ranges at Kilauea, repeat the distinctive, comprehensible style of Hawailan volcanism. Compared to the worldwide explosive volcanic events common to both geologic and human history, Hawaiian 
volcanism is reasonably well mannered and approachable. This was implicit in the action of Thomas A. Jagger, (1871-1953) a Massachusetts Institute of Technology professor, who establisied in 1912 the initial scientific facility that was to become tie Hawaiian Volcano Observatory (HVO), at the summit of Kilacea. Hivo has gathered and interpreted an extraordinary body of knowledge about the mobile magmas and lava that continue to build kilaue and the Hawaiian volcanic chain in the mid Pacific. The U.S. Geological Survey (USGS), having staffed HVO since 1947, has led this scientific achievement. In 1987, marking the 75th anniversary of HVO, the USGS published a large, two volun: compendium entitled Volcanism in Hawail, Professional Paper 1350 (Decker, et al., 1987). There was no intent to examine the geothermal energy potential of kilauea amidst the many scientific objectives of this excellent collection of papers. Horever, the papers in Professional Paper 1350 are important supplements to a thin geothermal drilling and production data base for any evaluation of the geothermal resource which exists in the East Rift zone of Kilauea. (Professional Paper $1350^{\circ}$ may be examined or purchased at the Earth Science Information Center, USGS, 504 Custom House, 555 Battery street, San Francisco, CA 94111. Telephone 415-556-5627.

The vertical magma conduit under the summit of the kilauea volcano is the central feature of a vigorous construction process. A catalog of 20,000 earthquakes, collected by HVO since 1962, reveals in substantial detail the active processes of magma transport within Kilauea's structures (Klein, et al., 1987). Long period earthquakes trace both conduits and magma bodies rising from 60 kilometers depths to a shallow magma reservoir between 3 and 7 kilometers below the sumit caldera floor. The reservoir is aseismic because it stores a relatively large mass of hot liquid charges of rising magma until an eruptive event is initiated at the summit or the magma moves laterally into linear $r$ ift zones for further underground distribution. The openings into kilauea's two 
active rift zones are near the upper limit of its summit ragma reservoir. The solid roof of both the reservoir and the iatera: conduits show varying levels of seismicity which reflects magma mass and transport at greater depth. The long linear rift zones, radiating from the summit reservoir, effect a fundamensi, horizontal, internal distribution of magma away from a volcanic center. A tensional stress field, across the rift zone, facilitates magma emplacement commonly driven downrift bi the hydrostatic head gained from its brief residence in the sumit reservoir.

The Hawaiian volcanic ift zones are created as the roofs and surface expression of active deep magma conduits. Both transient and locally stored magma masses establish an abundance of thermal energy. Specifically, it is the repetitive process of magma emplacement as near vertical dikes in the tensioned roof rocis which creates the heat source for a geothermal resource potential in an active rift zone. The kilauea East Rift zone (KERZ) is in a vigorous stage of growth with a geologically optimal level of internal magma activity. It is flanked by an abundant groundwate: regime on the north and by the sea on the south. The junction of abundant heat and fluids along the KERZ establishes its unique geothermal resource potential.

\section{A.2 RILAUEA EAST RIFT ZONE AND ITS GEOTHERMAL RESOURCE POTENTIAL}

The topographic form of the KERZ, after its gradual emergence from Kilauea's gentle summit rise, is that of a broad, linear ridge. The ridge crest courses east-northeast and straight for 42 kilometers, from an elevation of 880 meters at Makaopuhi Crater to sea level at Cape Kumukahi (see Figure A-2). Beyond the Cape, the submarine element of the KERz carries the same straight course for an additional 70 kilometers to termination on the ocean floor at an approximate depth of 4,800 meters. The entire structure, subaerial and submarine, was built rapidly by repeated rift crest 
lava eruptions supplied by magma transport in the underiy:ng conduit. In the middle of the subaerial element the lava apron has a maximum topographic width of 18 kilometers measured roma: to the rift axis. The more significant feature of the KERZ is the crestal band of local volcanic cones, craters, linear fissures and graben fault structures that reflect the crestal, cross $z i f$, tensional stress above the deep magma conduit. The surface width. of this active band is approximately 3 kilometers.

In 1976, at a location approximately 10.5 kilometers uprift fron Cape kumukahi and on the active crest of the KERZ, the initial geothermal test well, HGP-A, was drilled to a total depth of 1966 meters. A bottom hole temperature of $358^{\circ} \mathrm{C}$ was encountered and a total mass flow rate of 110,000 pounds per hour, 43 percent steam and 57 percent liquid, was measured. Following installation of a 3 MW turbine generator in March 1982, the steam production of this initial well has provided electric power in the range of 2.8 to 2 Mw. Except for scheduled overhauls, this small geothermal porer plant has operated continuously for seven years with an availability factor of approximately 90 percent. The geothermal fluid and electrical production from this single well and plant, now called the HGP-A Generator Facility, is discussed in more detail in section A.5. This achievement provides the most meaningful indication of an exploitable geothermal resource in the KERZ.

The internal fabric of fast-building Hawailan rift zones is a nearly horizontal, planar sequence of submarine and subaerial lava flows. These basaltic flows originate from local volcanic vents or parallel linear fissures situated along the rift crest overlying the deep magma conduit. In the upper part of the KERZ the top of the magma conduit appears to be shallower (seismicity to 2-3 kilometers) and consistently open (deeper aseismic zone) as discussed in Hardee, 1987. The continuous lava eruption which began in January 1983 in the upper KERZ, is now venting from a 
newly constructed volcanic cone, $C 48$, at a point approxinatei $\because .0$ kilometers down rift from Makaopuhi Crater. The lava flons are spiling southeastward and into the sea between Kupapau and iakmia Points.

In the lower 30 kilometers of the KERZ the top of the nagra conduit appears to be deeper (about 3.4 kilometers or more) and more commonly closed. Here, the advancing magma reopens conduits by the hydraulic injection capability of its significant flija pressure. The existing host rock is penetrated by the mobilo fluid magma in nearly vertical planar sheets, several feet thick. Both thermal energy and high temperatures are maintained by repetitive dike intrusion and solidification. This dike building process is facilitated by the tensional stress imposed on the rift crest, from magma conduit depths to the surface, by earthquakes, normal faulting and slumping of the seaward south flank of the KERZ. Dike emplacement in the lower KERZ efficiently transfers. high heat quantities from magma to shallower prospective geothermal reservoir intervals, as shown. in Figure $A-3$.

Because Hawaii geothermal drilling records, required to be filed with the State of Hawaii Department of Land and Natural Resources (DLNR), are reported in English units, the following discussion will utilize the same. The productive geothermal well HGP-A has a 7 inch perforated Iiner completion in the depth interval between 2920 and 6450 feet. This interval of submarine lava flows and younger intrusive dike rock presented a temperature profile that increased to approximately $620^{\circ} \mathrm{F}$ at 4000 feet, decreased to about $570^{\circ} \mathrm{F}$ at 5800 feet and increased to a maximum $676^{\circ} \mathrm{F}$ at $6450-$ foot total depth (see Figure A-4). The selection of the top of the geothermal reservoir (and completion) interval in this first well seems debatable. A restriction in the liner, just above 4000 feet, unfortunately precludes a spinner evaluation of the deep fluid entries in HGP-A. Aside from these concerns, this well 
continues to produce geothermal reservoir fluids oith littie decline since it was put into production in December 1981.

Puna Geothermal Venture (PGV) during 1981-85 drilled three offset welis (about 1800 and 3500 feet away from HGP-A well). In a publicly distributed November 1987 Environmental Impact statement (EIS) for a proposed $25 \mathrm{MW}$ (net) geothermal power plant a:ai wellfield, PGV states the geothermal reservoir extends belo: 4000 feet. PGV bottomed these offset wells at total depths betrieen 7300 and 8000 feet. The EIS briefly characterizes the geotherma: reservoir as "very high temperature (over $600^{\circ} \mathrm{F}$ ), two-phase (vapor-liquid)". Higher steam fractions were obtained in all PGi initial flow tests than the 43 percent steam fraction long prevailing in the HGP-A well production. From these four wells which have produced or tested geothermal fluids, the geothermal resource, in the kapoho locale of the lower KERZ, is a $600^{\circ} \mathrm{F}$, two-phase regime at moderate depth. Three additional exploratory geothermal wells drilled along the south edge of the KERz crestal structure have encountered encouraging temperatures but have not demonstrated fluid yielding reservoir intervals by flow tests These seven deep geothermal wells are discussed in more detail in Section A. 5 following.

DLNR, under its published Rules on Leasing and Drilling of Geothermal Resources, requires the filing of certain well reports (s13-183-85) following completion of drilling operations on any geothermal well. After an initial period of confidential status, these well records are opened to public access. The reports of all seven of the geothermal wells drilled in the lower KERZ may now be copied or examined at the public document room. Significant documents include well drilling and completion histories, lithologic and temperature logs, some geophysical logs and water sample analyses. 
A hypothetical geothermal reservoir would be expected so be located in the tension stressed, fractured rock below the cresta? band of the KERZ as shown in cross section in Figure $A-j$. Fas moderately deep vertical extent of the reservoir would be positioned in the hot diked roof above a deeper bundie of magra conduits or a possible static magma body. penetrations of copious supplies of fresh groundwater, and of seawater to a lesser extent, would enter at depth from opposing boundaries of the fractured reservoir to $\mathrm{mix}$ in an internal convection cell with a base temperature of $600^{\circ} \mathrm{F}$. The cross rift and long rift extent and the specific nature of the effective side boundaries of the hypothetical reservoir have yet to be determined. Drilling along the south flank of the KERZ crest suggests that sharp vertical boundaries exist there.

Subsurface supplies of waters that would contribute to KERZ geothermal regimes are inferred to be large. The two shield volcanoes, Mauna Kea and Mauna Loa draw heavy precipitation Erom the northeast trade winds. Annual rainfall of 100 to 125 inches is received on the lower slopes of Mauna Loa and the crest of the KERZ. Practically all of this sinks into the porous surface lavas and this meteoric water infiltration has established a very large ground-water body along the whole north flank of the KERZ. A limitless supply of seawater can infiltrate the entire narrow southern Elank.

In spite of the paucity of specific hydrologic subsurface data, several early findings suggest that interactions between groundwater, geothermal fluids and seawater will be intricate. A small group of private water wells, drilled on the lower KERZ before its geothermal potential was perceived, were never utilized because of the poor quality of the abundant shallow groundwater found. This has recently been identified as natural degradation caused by leakage from the now proven geothermal reservoir (Iovenitti, 1987). In the produced liquid fraction from the HGP-A 
well, the sodium to chloride ratio within the steadily increasing total dissolved solids content indicates seawater intrusion into the geothermal reservoir. A preliminary working concept of iarae fresh water and seawater supplies aggressively penetrating tie prospective geothermal core of the KERZ and contributing to tre hot fluid convection is sketched in Figure A-5.

\section{A.3 LEGAL STATUS AND REGULATION OF GEOTHERMAL RESOURCES}

Ownership. of geothermal resources is claimed by the state of Hawail under state lands and under Reserved lands. The latter are lands owned or leased by any person in which the state or its predecessors in interest has reserved to itself, expressly or by implication, the minerals or right to mine minerals, or both. Most private land ownerships in the KERZ are Reserved lands. Certain private land owners may eventually choose to test in the courts the state's claim to geothermal resource ownership related to the Reserved land concept. All geothermal resource develcpment commonly will require dual leases on the Reserved land tracts to be utilized. A geothermal mining lease must be obtained from the State for the subsurface rights to the geothermal resource and a lease must be obtained from the landowner for surface access and utilization. DLNR administers geothermal leasing and drilling under rules in Title 13, Chapter 183, which were approved in June 1981. Key state geothermal leasing provisions are royalties of 10 to 20 percent on resources produced, sold, or utlizized. When

necessary to initiate or continue commercial production of geothermal resources, the state Board of Land and Natural Resources (BLNR) is authorized to waive royalty payments to the state for any period up to eight years. Ten year primary terms of leases are extendable to a total of 65 years. Individual lease tracts are limited to 5000 acres of contiguous lands. State lands are leased by public auction only, and Reserved lands are leased by grant to the landowner or by auction. Hawaiian land ownership tracts, highly varied in size and in shape, are legally 
represented by Tax Key Maps. Land corner monuments and slizeys oE the highly varied tracts have not been commonly utilizad.

Hawaii has stringent land use laws (see Hawaii Revised Statut:s 205) which, when enacted, did not address geothermal resource utilizations. The BLNR and the counties jointly establish aril regulate land use districts which are dedicated to urban, rural, agricultural and conservation uses. In 1984, state reguiations were amended to enable geothermal development in all land use districts provided a Geothermal Resource Subzone (GRS) was firs: established by procedures under Title 13, Chapter 184. The Bry? was given the authority to designate and regulate GRS. Three GRS were approved and established in the KERZ as shown in Figure A-6. The total amount of lands included are approximately 21,900 acres apportioned among three blocks as follows:
Kilauea Lower East Rift (Kapatio Section)
7,353 acres
Kilauea Lower East Rift (Kamaili Section)
5,531 acres
Kilauea Middle East Rift
9,014 acres

Geothermal development may proceed only within such desigrated GRS areas. Proposed designations of new GRS may be initiated by the BLNR, any landowner, geothermal lessee or lease applicant, as can proposed modifications and withdrawals of existing GRS. Environmental impact statements are not required in designating, modifying or withdrawing any GRS. The GRS process has structured the deliberations about possible geothermal resource utilizations and has interfaced the county and state authorities within designated GRS areas of the KERZ. Hawaii County is the lead authority if exploration or development is proposed on rural and agricultural lands within the GRS. DLNR is the lead authority on conservation lands within the GRS. It must be noted that public input to the GRS process is significant. Until the value of: geothermal enterprise is more clearly demonstrated, resistance to GRS enlargement in the $\mathrm{KERZ}$ is expected.

$$
A-10
$$


The approval paths for exploratory geothermal driliing on dus: surface and subsurface (state) leases within GRS are briefly ci $=\equiv d$ here because this is the critical, near term activity requirec in the KERz. Permit requirements in the Geothermal Resource Subzones are detailed in Appendix B. On agricultural and rural lands a Geothermal Resource Permit (special use permit) must be approved under Rule 12 by the Hawaii County Planning Commission. On conservation lands, a plan of Operation and a Conservatica District Use Permit must be approved by DLNR. Individual dilinin permits for each proposed geothermal well are required from DLVR regardless of the land use classification of the drillsite.

\section{A.4 AVAILABILITY AND ACCESSIBILITY; PROSPECTIVE AREAS}

The three designated GRS areas in the lower KERZ cover a substantial portion of the prospective crestal trend which extends for approximately 30 kilometers between the $c 48$ erupting volcanic vent and Cape Kumukahi. DLNR records of issued geothermal mining leases and applications for lease now cover a substantial portion of the GRS areas. Several of the large landowners in the KaRz are lessees under issued geothermal mining leases. Kapoho Land and Development Company, Bishop Estate and the Campbell Estate are landowners with geothermal leases or applications dedicated to existing exploration or development agreements with certain geothermal operators. Other privately owned land tracts within the GRS areas are, or may be, under lease or option agreements with geothermal operators. Such leases may or may not coincide with issued mining leases and may or may not be disclosed in public records.

HECO and its consultants made no inquiries or evaluations of landowner and leaseholder positions in contemplating or structuring this RFP. Landowners, leaseholders and geothermal operators positioned in the KERZ will determine their individual responses to this RFP. Proposers are cautioned that they proceed 
at their full risk in evaluating and responding to the status $=$ lands and leases in the RERZ.

off-road accessibility in most of the KERZ terrain is dieficilt: =0 impossible, even for four wheel drive vehicles. Dense undergrowth, forest cover and impassable lava rock surfaces are typical barriers. Most private land tracts are fenced or posted against trespassing. New road construction approvais Eor geothermal development will be keyed to the status of the land traversed: agricultural, rural or conservation.

\section{A.5 ELECTRIC GENERATION AND RESOURCE PRODUCTION IN THE KERZ}

The 3 MW power plant of the HGP-A Generator Facility was constructed in 1981 with funds jointly provided by the U.S. Department of Energy, the state and the County of Hawail. A profile of the plant's electric generation history is shown in Figure A-7 for the seven year interval, commencing in March 198 ?, of commercial power delivery to Hawai Electric Light company. Because of economic constraints, detailed well production records were not accumulated. Possible declines in wellbore deliverability or reservoir performance might be inferred fron generator outputs; an initial peak output of $2.8 \mathrm{MW}$ versus $2.45 \mathrm{MW}$ currently suggest a 1.8 percent annual decline in well production. Although several scheduled overhauls were made without finding serious degradation, certain material and equipment deficiencies in plant design have been clearly demonstrated and may be registered in the output decline. Cumulative silica scaling in the HGP-A wellbore may be a contributing cause of the apparent decline. Several very informative studies of plant and well performance have been completed and documented in recent years by Donald Thomas of the Hawaii Insitute of Geophysics.

The continuous 7-year geothermal fluid production of the EGP-A well has been very successfully utilized. However, it has

$$
A-12
$$


afforded only a meager basis for understanding the geatherma resource. The lack of detailed records of fluid product: parameters, of periodic pressure-temperature-spinner surveys ova: the well's 3530-foot perforated liner completion interval and of reservoir pressure monitoring in any offset observation hole are to be noted. This provides little context within which severa! perceptive and thorough studies of produced fluids chemistry can be conclusively judged (Thomas 1985a and 1987).

The total mass flow of HGP-A well, measured initialiy as approximately 47,300 pounds per hour steam and 62,700 pounds per hour liquid, is a product of wellbore mixing (inside 7 inch production casing) of different fluids from multiple, separate entry points of imprecise depths, pressures and temperatures. The distinctive, low salinity of the first produced liquid, suggestive of a meteoric water dominance in the geothermal reservoir, was lost in a gradual, four-year increase in salinity, to about 15,000 $\mathrm{mg} / \mathrm{kg}$ of NaCl, with production for electric generation. The Na and $\mathrm{Cl}$ ionic ratios and other metallic changes seem to prove a seawater intrusion into HGP-A well's production sink. This fluid change to a new high but stable, level of salinity appears to confirm the implications of an irregular presence of anhydrite filled fractures amidst other alteration minerals found in the HGP-A rock cores from the reservoir interval. Fracture guided intrusions of seawater into the geothermal fluid convection cells must repeatedly occur. However, these intrusions individually are probably limited in duration and volume because of rapid self-sealing by new mineral deposition at the seawater-geothermal fluid interface. A diminution of $\mathrm{pE}$ from 7.6 to 6.5 , attending the increase in salinity of produced brines, was measured. Possible minor decreases in produced steam fraction and wellhead temperature, if suspected from time to time in short term flow variations, have not been measured to identify any long term trend. The precisely identified stability of the silica content of the brine (about $800 \mathrm{mg} / \mathrm{kg}$ ) and of the low content of 
non-condensible gas ( 0.3 percent by weight) in the stean phom reflect the apparent stability of the total mass flow produced a! the HGP-A well since December 1981.

Key information from the seven deep geothermal wells drilied into the geothermal reservoir, or equivalent depths, in the lower $\mathrm{k} \equiv z \mathrm{z}$ is summarized in Table A-2. Their locations are shown on EigurE A-6.

Key fatures of the wells which penetrated the geotinormal reservoir were $95 / 8$ inch production casing (cemented just belor. 4000-feet in KS wells) and 7 inch perforated liner in an $81 / 2$ inch hole to total depth. It should be noted that both HGP-A and $\mathrm{KS}-1$ wells include remedial 7 inch casing inserts that were emplaced before production and testing. The ks 1 and 2 tests support the recent conclusion (Thomas, 1987) that a dry steam producing zone exists in the HGP-A well. Composite chemical data from the four wells tested are presented in Tables $A-3$ and $A-A$.

Final Hawaii County approvals are being sought for the Geothermai Resource Permit for the PGV's proposed 25 MW (net) geotbermil plant and wellfield which expectedly will include KS-IA and 2 wells in production service. Drilling plans for the requirea additional production and injection well are in preparation for a commencement of development operations later in 1989.

A proposed Scientific Observation Hole program at additional locations within the GRS areas along the lower KERZ is planned. The intended slim hole drilling program, utilizing both rotary and diamond core procedures, is jointly funded by the state of llavaii and geothermal operators (Geothermal Resources Council Bulletin, 1988). Information from the intended 4000 -foot holes is to be promptly released to the public domain and should be available during the negotiation period for the Power Purchase Agreement. 


\section{A.6 GEOTHERMAL RESERVOIR POTENTIAL IN THE KERZ}

The geothermal reservoir potential of the KERZ is most strongly supported by the HGP-A Generator Facility perforinance combin. with its position above a magma conduit which is reasorabi: defined as to location and function. The critical concern is ar estimate of the magnitude of this reservoir potential within tie lower KERZ between the $C 48$ vent and Cape Kumakahi ( 30 kilometsis or 18.6 miles).

Volcanic eruptive history proves recurring magma transport through the entire lower KERz. Significant lava eruptions from Heiheiahulu "in the reign of Arapai" - circa 1750 A.D. (vent is 22 kilometers SW of Cape Kumakahi) and the Kapoho eruptions of 1955 and 1960 obtain importance against a detailed modern study of Kilauea's magma balance. The USGS - HVO concludes that nearly 50 percent of all magma mass remains below ground, being emplaced as intrusive dikes and sills. The entire KERz has become a more favored structure for magma distribution and dike construction since the magnitude 7.2 Kalapana earthquake of 1975 which tensionally opened the entire KERZ structure by seaward slumping of its south flank, as shown in Figure $A-8$ and discussed by Lipman, et al.. 1987. A preliminary estimate, made from deflations of Kilauea's summit following the 1975 quake, was that 3 million cubic meters per month of magma was moving into the rift zones. The deep fracturing in the kERz consequent to this major earthquake should enlarge or maintain reservoir permeability and new meteoric and seawater inputs to geothermal fluid convection cells. Heat, fractures and fluids are renewed in the dynamic, continuous structure above the KERZ magma conduit.

The 500 MW objective of this RFP is based on market considerations (Lesperance, 1988, and Department of Business and Economic Development, 1989). No integrated study exists of all the KERZ geoscientific and well data that would provide a creditable 
estimate of the total geothermal potential. Only additicial drilling, flow testing and production can provide measures o: tra energy capacity that is indicated to exist in the GRS of the KERz. It is of some interest to note that one existing developer inters. to utilize a 500 acre land area dedicated to its 25 Mi (:ns) generation capacity. This suggests that the 22,000 acres $y i$ inin the three GRS areas, if only 50 percent productive, could yieic 550 MW OE capacity.

\section{A.7 GASEOUS AND LIQUID WASTE DISPOSAL FROM GEOTHERMAL WELLFIELD ACTIVITIES}

Effluent waste disposal from the producing HGP-A well has not deen managed in a way that is acceptable for future geotinernal development in the KERZ. The 57 percent brine fraction, carrying about $15,000 \mathrm{mg} / \mathrm{kg}$ of $\mathrm{NaCl}$ and $800 \mathrm{mg} / \mathrm{kg}$ of $\mathrm{SiO}_{2}$, is discharged to shallow surface ponds for percolation into the ground. The attending silica deposition eventually precludes percolation and new ponded areas are then utilized. This practice is unacceptabie for the Euture commercial development that will occur along the KERZ. The produced non-condensible gas (NCG) is burdened with about $850 \mathrm{mg} / \mathrm{kg}$ of $\mathrm{H}_{2} \mathrm{~S}$. Normal plant operation produces 1 i00 pounds per day of $\mathrm{H}_{2} \mathrm{~S}$ that is now abated, with reasonabie reliability, with $\mathrm{NaOH}$ in a two stage scrubber and by incineration. The $\mathrm{H}_{2} \mathrm{~S}$ abatement experience at HGP-A, although costly and problem-plagued, provides notice that reliability, reserve capacity and alternate options of $\mathrm{H}_{2} \mathrm{~S}$ mitigation will be essential to successful "good neighbor" geothermal development in the KERZ. It is appropriate to note that PGV's Amended Application for Geothermal Resources Permit for $25 \mathrm{MW}$ (net) plant and Wellfield (December 1988 submittal to Hawaii County Planning Department) proposes the injection of recombined streams of brine, condensate and NCG back into the geothermal reservoir. Just such recombined fluid injection reportedly is successful in its first year of utilization in the coso geothermal field in california.

$$
\text { A-16 }
$$


The present status of $\mathrm{H}_{2} \mathrm{~S}$ emission controls, regarding geothermal development in the KERZ, merits special attention. A 1982-1983 state survey of $\mathrm{H}_{2} \mathrm{~S}$ levels in a 27 station KERZ grid vas completed, as were local surveys by HGP-A and PGV. These surye $3:$ should provide some insight into natural $\mathrm{H}_{2} \mathrm{~S}$ emissions froni continuous volcanic gas venting that proceeds between the obvious eruptive events. Aside from this singular feature of the KERz, the Hawaii Department of Health (DOH), as the state regulatory authority, is now proposing a statewide ambient 1-hour emission standard of 139 micrograms $\mathrm{H}_{2} \mathrm{~S}$ per cubic meter (0.1 ppmv) for inclusion in Administrative Rules Chapter 11-59. DOH a!so proposes a statewide allowable increment of $0.35 \mathrm{mg} / \mathrm{m}^{3}(0.35 \mathrm{ppmv})$ of $\mathrm{H}_{2} \mathrm{~S}$ emission from any new facility. This proposal and lesser $\mathrm{H}_{2} \mathrm{~S}$ constraints are included in draft DOH Rules 11-60-15 and 16 .

An additional DOH regulatory authority extends statewide to underground injection control (UIC). Though the non-potable quality of ground water was proven by landowner driliing in the KERZ before recognition of the geothermal resource, some of the GRS areas remain in the Underground Sources of Drinking Water (USDW) status. Injection of produced geothermal fluids will require approval by the DOH.

\section{A.8 VOLCANIC AND SEISMIC IMPACTS ON WELLFIELD DEVELOPMENT}

An excelient summary of the volcanic hazards that occur along the KERz is presented by Mullineaux, et al. 1987. Lava flows will pose the most likely hazard over time, as shown in Figure A-9. However, lava flows are controlled by topography, as any surface water flow would be. A careful evaluation of the KERz terrain can be made with the assistance of detailed topographic maps recently published by the usGs $(1: 24,000$ scale and 20-foot contour interval). The probable flow course and other possible topographic controls can be reasonably predicted. The morphology and emplacement dynamics of the blocky a type of lava flow are 
detailed by Lipman and Eanks, 1987. This more viscous, thickar building flow commonly moves in a 100-200 meter frontal visti, seieral meters high and at velocities up to 50 meters per hour. Final flow thickness may range from 5 to 10 meters in neigi...

The less likely but more serious volcanic hazard, the Eissure or vent eruption site event, is mitigated by the much smaller area oz direct impact. However, against the expected long life of the geothermal resource it cannot be considered predictable in tine or location. It will remain the greatest risk in development of che geothermal resources of the KERz. Air lofted tephra (rock debris) ash and gas concentrations from eruptions may yield a range of secondary and addressable impacts on any KERZ geothermal site depending on wind conditions and distance from source points. Ground surface dilation, extension or subsidence due to local magma movements or lava discharges, are additional processes common in the KERZ that are of minor impact on wellfieid operations.

The high seismicity of the KERZ is directly correlated with its high level of constructional volcanic activity. This is clearly presented in an excellent new map publication of statewide scope: "Seismicity of Hawaii, 1962-1985,. USGS Open File Report 88-285" which may be purchased at the Pacific Map Center, 647 Auahi Street, Honolulu, HI 96813, Telephone 808-531-3800. The seismicity of Kilauea's magma system, detailed by klein, et al., 1987, chiefly includes events of less than magnitude 4 which are generated by magma and dike activity in the 2-5 kilometers depth. interval. This class of seismicity presents a significant guide for geothermal wellfield development and presents little or no attendant hazards. It is the deep, infrequent, tectonic earthquakes of magnitudes \pm 7 which. could impact KERZ geothermai development. Fortunately, the largest historical earthquake in this class, the November 1975 magnitude 7.2 event, at a depth of 9 kilometers under Kalapana on the southeast coast of the Island of 
Hawaii, was fully recorded by the HVO seismic network. This imposed a 0.22 gravity acceleration measured at siio (43 kilometers NNW of Kalapana). Geothermal wells in the KERZ, with multiple cemented casing strings and series 900 wellheacs, spider braced in reinforced concrete cellars, should surpass the 0.4 gravity acceleration factor selected for the plant and sursace facility design to safely withstand the tectonic class of earthquake.

Significant strategies can be utilized for the protection of KERZ geothermal wellfield development and production operations. Directional drilling would permit wellheads to be clustered on elevated or cinder berm protected wellpads that would be at minimal risk from both volcanic and the seismic hazards. Drilling rigs may merit heavier guy-lines as added protection. Steam and other wellfield pipelines will be vulnerable to lava flows and to major earthquakes. Rapid cinder berm construction and pipeline repair capacities can be considered as response options.

The common volcanic-seismic basis of both the resource and hazards in the KERz should encourage development of key surveillance methods. A very sensitive seismic net could simultaneousiy forecast possible lava eruptions and track the wellfield production and injection fluid impacts to optimize geothermal reservoir management. Multiple physical and chemical parameters can be examined for volcanic-seismlc-exploitation correlations that may increase thermal energy recovery and reduce the attendent risks.

\section{A.9 GEOTHERMAL WELLS AND WELLEIELD CONCEPTS AND OPTIONS}

The important tasks in future geothermal drilling in the KERz will be to increase well productivity and reduce well costs. An early evaluation of directed, angled completion intervals seems appropriate, given the common feature of near vertical and planar 
fractures, partings and dikes parallel to the rift axis, in tie expected production intervals. The four penetrations of the Eli : i yielding reservoir to date were in vertical wellbores, which is less than an optimal orientation to intercept near verti:al openings. If an upper reservoir yield of 100 percent $3 c e s \ldots$ production could be achieved by more precise completions, possibiy in the 4000 to 6000 foot depth interval as suggested in the $3: 5$ wells, a productivity increase and associated cost reduction mign: significantly assist initial wellfield development. This Einding would next invite consideration of "big hole" production vielis.

In the context of improving well productivity and accuratei $;$ measuring the results, it is important to note that initial well flow testing of KERz geothermal wells is not a simple and low cost task (D'Olier and Iovenitti, 1984). The presence of cool groundwater aquifers to possible depths of about 2000 feet cails for gradual preparations. An initial static warmup (eirst geothermal fluids rising within the completion fluid coiumn of tho shut-in well) followed by accelerated heating and deliberate bleeding will elevate the wellbore to a more uniform thermal state to accommodate the initial high mass - high temperature flow upon opening. The capacity to go promptly to fully opened, vertically vented flow to atmosphere must be present because of an extremeiy erosive initial discharge of a sharp grit of rock and minerais from the producing formation. A continuous, full open flow, with its 120 decibel noise penalty, appears to be the most efficient, fast and safe procedure to obtain this critical well cleanup before shunting the flow into measurement runs and muffied venting.

As waste fluid injection is thoroughly evaluated and is considered for high utilization in the KERZ, the function and reliability of injection wells will become as critical to system operations as production wells are. Expecting a design and quality comparable to production wells, injectors must be further protected with a 
hang down casing string (replaceable) as the injectate conduit =0 the perforated linered interval at depth. Actually, marginai production wells may be placed on back up injection service i: the addition of a protective hang down string. It appears that accurate and detailed knowledge of geothermal reservoir performance and optimal utilization of every well will be essential in the KERZ.

\section{A.10 MATURITY OF TECHNOLOGY}

The improvement of geothermal well design and material selection will be important considerations for economic development of KERZ reservoirs. The conventional design and $\mathrm{K}-55$ grade of casing and liner used in the HGP-A well seems to be endorsed by more than seven years of continuous production. However, the down hole conditions of this wellbore are poorly known. The costs of the offset wells, at industry market rates in the early 1980's, commonly exceeded $\$ 2,000,000$ per well for drilling and completion. Substantial improvements in logistics and management of future development drilling should be important cost reduction factors. Upgrades in tubular materials, couplings, and possible cementing in tension procedures may provide gains on a benefit-to-cost basis. Modern rotary drilling, cementing and drilling fluid practices are mature practices that should serve efficiently in KERZ geothermal wellfields. ANSI 900 series wellhead equipment is indicated for standard utilization on KERZ production wells.

The production of two-phase fluid production and 100 percent steam production are mature geothermal industry technologies. High volume liquid injection into a producing geothermal reservoir is a developing technology in the industry. Injection into KERz reservoirs may prove difficult to integrate with the production objectives; an alternate injection disposal target may be deep seawater zones immediately south of the expected geothermal reservoirs. 
The substantial daily fluctuation of the Oahu power requiraments indicates that PROPOSERS should consider a load-following, da: $y$ cycling of KERZ geothermal wellfield production as one cption among other possible responses. Daily cycling in the form af a shared reduction of steam supply, from a welifield sector producing commonly to one generating plant, is not known to be a sustained practice anywhere in the geothermal industry at snis time. The required reduction alternatively might be achieved by $a$ nightly shut-in of a much smaller number of wells. The impacts $0:$ a common nightly reduction, or of a selected (or rotated) Euli shut-in, will relate to the magnitude of pressure and cemperature increases imposed in each wellbore, wellhead and flow control valve and to the endurance or quality of well design, materials and equipment. All of these factors will be site specific to tie geothermal reservoirs, producing wells and economics to be encountered in the KERZ.

\section{A.11 OPERATIONS AND MAINTENANCE}

Replacement (makeup) well drilling, redrilling for extended or improved production or injection service, and remedial cleanouts may become significant requirements in KERZ geothermal fields. No - other extraordinary requirements are indicated.

\section{A.12 REFERENCES FOR APPENDIX A}

Bel1, D. and Thomas, D.M., October, 1988. Report on the October, 1987 Hawaiian HGP-A Power Plant Overhaul and Reservoir Production Data, in Geothermal Resources Council Transactions. Vol. 12, p. 343 .

Clague, D.A. and Dalrymple, G.B., 1987. The Hawailan-Emperor Volcanic Chain, in U.S. Geological Survey professional Paper 1350 , p. 5 . 
Decker, R.W., 1987, Dynamics of Hawailan Volcanoes: An Overviev, in U.S. Geological Survey Professional paper 1350, p. 997.

Decker, R.W., Wright, T.L. and stauffer, P.H., Editors, 1987. Volcanism in Hawail, U.S. Geological Survey professional paper 1350, Volumes 1 and 2 .

Department of Business and Economic Development, 1989. Environmental Review, $500 \mathrm{MW}$ Geothermal Development within the Three Geothermal Resource subzones of the kilauea East Rift Zone, Puna District, Island of Hawaii. March, 1989:

D'Olier, W.L. and Iovenitti, J.L., 1984. Drilling and Testing Geothermal Wells in an Active Volcanic Domain, Puna Geothermal Field, Hawaii, USA, in Proceedings, ASEAN Geothermal Workshop, Banding, Indonesia, May 1984.

Geothermal Resources Council Bulletin, November, 1988, Funds Appropriated for Resource Assessment in Hawail, p. 12.

Hardee, H.C., 1987. Heat and Mass Transport in the East-RiftZone Magma Conduit of Kilauea Volcano, in U.S. Geological Survey Professional Paper 1350 , p. 1471.

Iovenitti, J.L., 1987. Geothermal Fluid Leakage, Lower East Rift zone kilauea Volcano, Hawail, in Abstract Volume and Poster Session, Eawaii Symposium on How Volcanoes Work, HVO 75 th Anniversary, Eilo, Hawali, January, 1987.

Iovenitti, J.L. and Dolier, W.L., 1985. Preliminary Results of Drilling and Testing in the Puna Geothermal system. Hawail, in Proceedings, Tenth Workshop, Geothermal Reservoir Engineering, Stanford University, January, 1985. 
Klein, F.W., Koyanagi, R.Y., Nakata, J.S., and Eanigana, N.R., 1987. The Seismicity on Kilauea's Magma System, in $\mathrm{I} . \Xi$. Geological Survey Professional paper 1350, p. 1019.

Lesperance, G.0., October, 1988, Geothermal Development in Jara:i, in Geothermal Resources Council Transactions, Vol. 12, p. 75.

Lipman, P.W. and Banks, N.G. 1987, AA Flow Dynamics, Malina jos 1984, in U.S. Geological Survey Professional Paper 1350, p. 1527.

Lipman, P.W., Lockwood, J.P., Okamura, R.T., Swanson, D.A., and Yamashita, K.M., 1985, Ground Deformation Associated with the 1975 Magnitude - 7.2 Earthquake and Resulting Changes in Activity of Kilauea Volcano. Hawail: U.S. Geological Survey Professional Paper 1276. 45 pages.

Malahoff, A. 1987, Geology of the sumit of Loihi Submarine Volcano, in U.S. Geological survey Professional Paper 1350, $p$. 133.

Mullineaux, D.R., Peterson, D.W. and Crandell, D.R. 1984, Volcanic Hazards in the Hawaiian Islands, in U.S. Geologicai Survey, Professional Paper 1350, p. 599.

Thomas, D.M., 1985a, HGP-A Well Chemistry in Chemistry, Scale and Performance of the Hawaii Geothermal Project - A Plant, Electric Power Research Institute AP-4342, Project 1195-12, Final Report.

Thomas, D.M., 1985b, The HGP-A Generator Facility, Reservcir Characteristics and Operating History, in Proceedings, Electric Power Research Institute, Geothermal Workshop, 1985.

Thomas, D.M., 1987, A Geochemical Model of the Kilauea East Rift Zone, in U.S. Geological Survey Professional Paper 1350, P. 1507. 
Thomas, D.M. and Olson, H.J., 1989, Current status and Futur Research Objectives for the HGP-A Generator Facility, in Proceedings, Fourteenth Workshop, Geothermal Reservoir Engineering, stanford University, January, 1989 (in press). 
TABLE A-I

ISLAND OF HAWAII VOLCANIC CENTERS

Oldest Lava

- or Flow Dates*

Volcano

(years age)

Eruptions

Seismic:

Kohala

700,000

$\mathrm{K}-\mathrm{Ar} \max$

Last event

60,000 years

ago

Mauna Kea

375,000

$\mathrm{K}$-Ar max

Last event 4500 years ago

Hualalai

106,000

$\mathrm{K}-\mathrm{Ar} \max$

1800 A.D.

Minima I

Mauna Loa

400,000

$\mathrm{K}-\mathrm{Ar} \max$

38,000

C14 max

37 events

1832-1984

Occasional

Kileaua

23,000

C14 max

64 events

1790-1989

High continuous

since 1983

Loini

Fresh tholeite flows at summit,

Per swarm?

Shallow

swarms

Age?

$1971-75-84$

* K-Ar Potassium-Argon dating

C14 Radiocarbon dating 
TABLE $A-2$

KERZ DEEP GEOTHERMAL WELLS

\begin{tabular}{|c|c|c|c|}
\hline We11 & $\begin{array}{l}\text { Total } \\
\text { Depth } \\
\text { (feet) } \\
\end{array}$ & $\begin{array}{l}\mathrm{BHT} * \\
(\circ \mathrm{OF}) \\
\end{array}$ & Comments \\
\hline Ashida 1 & 8300 & 619 & No permeability or fluids; suspendeci \\
\hline $\mathrm{HGP}-\mathrm{A}$ & 6450 & 676 & $\begin{array}{l}\text { Producing } \pm 110,000 \text { lbs/hr TMF since } \\
\text { Dec } 81 \text {; about } 43 \text { percent steam and } \\
57 \text { percent brine }\end{array}$ \\
\hline $\begin{array}{l}\text { Kapoho } \\
\text { State } 1\end{array}$ & 7290 & 642 & $\begin{array}{l}\text { Short test: } 72,000 \text { Ibs/hr steam; } \\
\text { suspended }\end{array}$ \\
\hline $\begin{array}{l}\text { Kapoho } \\
\text { State } 2\end{array}$ & 8005 & 648 & $\begin{array}{l}\text { Short test; } 33,000 \text { Ibs/hr steam; }{ }^{*} \\
\text { suspended }\end{array}$ \\
\hline $\begin{array}{l}\text { Kapoho } \\
\text { State } 1 \mathrm{~A}\end{array}$ & 6562 & 572 & Tested; data proprietary; shut in \\
\hline Lanipuna 1 & 8389 & $685+$ & $\begin{array}{l}\text { Low perm., trace of fluids; } \\
\text { abandoned }\end{array}$ \\
\hline $\begin{array}{l}\text { Lanipuna } 1 \\
\text { redrill }\end{array}$ & 6299 & 300 & $\begin{array}{l}379^{\circ} \mathrm{F} \text { maximum; no fluids; } \\
\text { abandoned }\end{array}$ \\
\hline Lanipuna 6 & 4956 & $250+$ & $\begin{array}{l}\text { Major L.C. zone below } 4285^{\circ} \\
\text { suspended }\end{array}$ \\
\hline
\end{tabular}
* Bottom hole temperature Table modified from Thomas, 1987
* see Iovenitti and D'olier, 1985

Well locations are shown on Figure A-6 
TABLE A-3 GEOTHERMAL FLUID CHEMICAL COMPOSITION COMPOSITE DATA ${ }^{2}$

\begin{tabular}{|c|c|c|}
\hline Element & $\begin{array}{l}\text { Brine } b \\
(\operatorname{ppm}(w))\end{array}$ & $\begin{array}{l}\text { Steam } \\
\text { condensate } \\
(\operatorname{ppm}(w))\end{array}$ \\
\hline $\mathrm{Na}$ & $600-10,000$ & 0.17 \\
\hline $\mathrm{K}$ & $123-2,700$ & 0.10 \\
\hline $\mathrm{Ca}$ & $40-920$ & 0.10 \\
\hline Mg & $1-2$ & $<0.1$ \\
\hline $\mathrm{Fe}$ & $<1-8.4$ & 0.05 \\
\hline $\mathrm{kn}$ & $<1-8: 5$ & -- \\
\hline$B$ & $4-11$ & $<0.05$ \\
\hline $\mathrm{Br}$ & $40-80$ & - \\
\hline$I$ & $<20$ & -- \\
\hline$F$ & $0.2-0.9$ & -- \\
\hline $\mathrm{Li}$ & $1-9$ & $<0.01$ \\
\hline $\mathrm{Cl}$ & $925-21.000$ & $<2$ \\
\hline $\mathrm{NH}_{3}$ & $<0.01-0.1$ & 0.12 \\
\hline $\mathrm{SO}_{4}$ (c) & $9.2-24$ & 13 \\
\hline $\mathrm{Hg}$ & $<0.001-<0.05$ & - \\
\hline As & $0.09-0.4$ & $<0.01$ \\
\hline$s=(d)$ & $5-100$ & -- \\
\hline Total Alkalinity & $<10$ & $<10$ \\
\hline $\mathrm{HCO}_{3}$ & $0-18$ & 0 \\
\hline $\mathrm{CO}_{3}$ & 0 & 0 \\
\hline $\mathrm{SiO}_{3}$ & $420-1,500$ & 0.7 \\
\hline TSS & 70 & -- \\
\hline TDS (e) & $2,500-35,000$ & 15 \\
\hline $\mathrm{pH}$ & $<5-5.5$ & 3.5 \\
\hline $\begin{array}{l}\text { Conductivity } \\
\text { (mho/cm) }\end{array}$ & $3,100-67,000$ & 120 \\
\hline Density & 1.03 & $-\infty$ \\
\hline
\end{tabular}

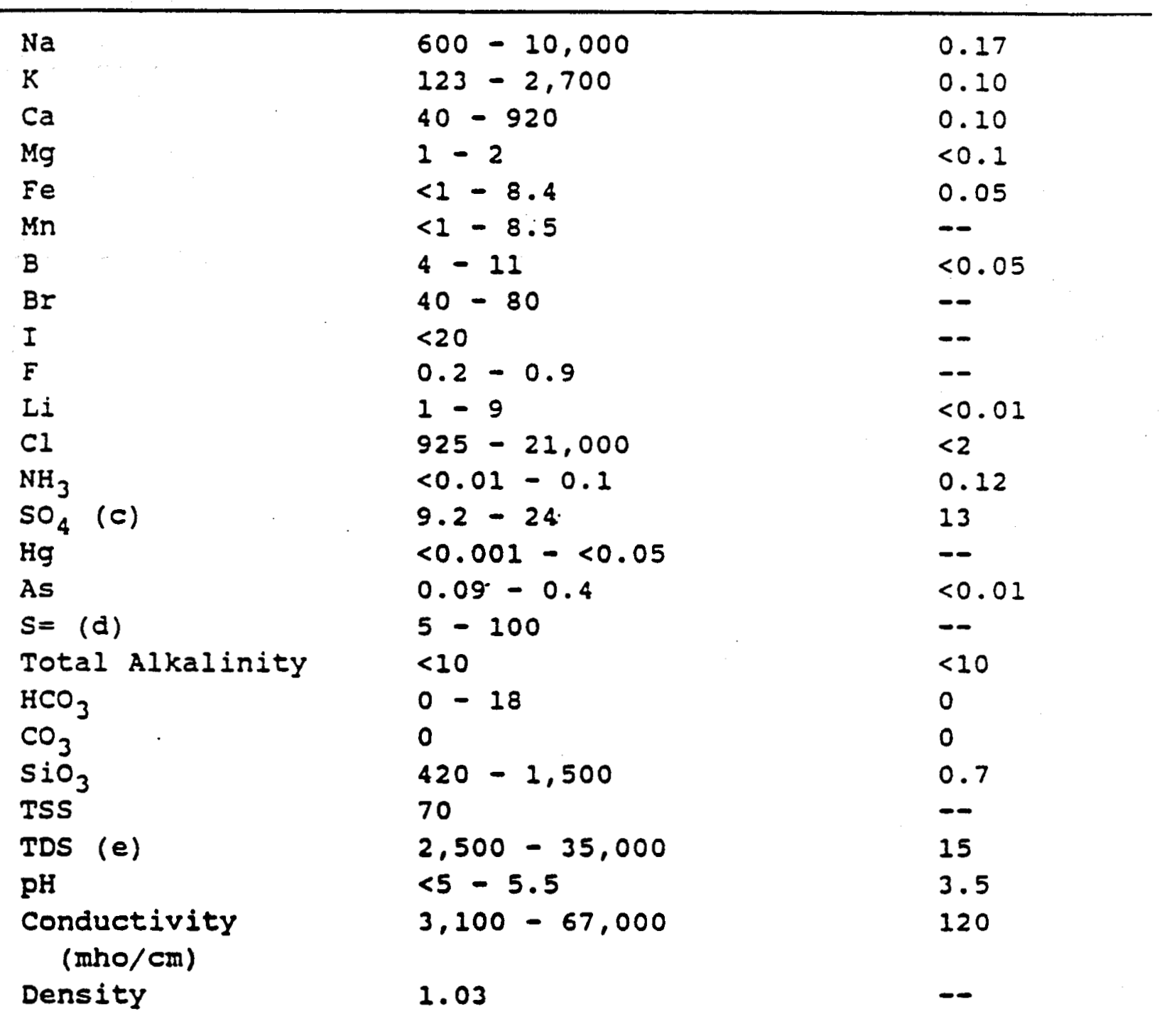

Composite data from three wells on the PGV site (KS-1, KS-IA, and $\mathrm{KS}-2$ ) and the HGP-A well.

b Wellhead pressure (WHP) $=155$ psig; Wel Ihead Temperature (WHT) $=368^{\circ} \mathrm{F}$.

C concentration high due to oxidation of $s=$ to $\mathrm{SO}_{4}$.

d Concentration low due to oxidation of $\mathrm{S}=$ to $\mathrm{SO}_{4}$.

e TDS = Total Dissolved Solids.

(from Department of Business and Economic Development, 1989) 
Table A-4 NONCONDENSABLE GAS COMPOSITION COMPOSITE DATA ${ }^{2}$

\begin{tabular}{|c|c|c|}
\hline Gas & $\begin{array}{l}\text { Observed } \\
\text { Steam Content } \\
\operatorname{ppm}(w)\end{array}$ & $\begin{array}{c}\text { Plant Design } \\
\text { Composition } \\
\text { ppm (w) }\end{array}$ \\
\hline
\end{tabular}

\begin{tabular}{lcc}
\hline $\mathrm{CO}_{2}$ & $250-1,042$ & 956 \\
$\mathrm{H}_{2} \mathrm{~S}$ & $800-1,300$ & 1950 \\
$\mathrm{NH}_{3}$ & $(\mathrm{c})$ & - \\
$\mathrm{Ar}^{2}$ & $6-13$ & 582 \\
$\mathrm{~N}_{2}$ & $10-700$ & - \\
$\mathrm{CH}_{4}$ & $($ d) & - \\
$\mathrm{He}$ & $11-140$ & 12 \\
$\mathrm{H}_{2}$ & $<009$ & \\
\hline Total NCG & $1,500-2,200$ & 3500 \\
\hline
\end{tabular}
a composite data from three wells on the PGV site (KS-1, KS-1A, and $\mathrm{KS}-2$ ) and the KGP-A well.
b WHP $=155$ psig: WHT $=368^{\circ} \mathrm{F}$.
C Below Detection Limit ( $<1.5 \mathrm{ppm} \mathrm{NH}_{3}$ in $\mathrm{ks}-1 \mathrm{~A}$ ).
d Below Detection Limit (<0.2 $\mathrm{ppm} \mathrm{CH}_{4}$ in $\mathrm{ks}-1 \mathrm{~A}$ ).

(from Department of Business and Economic Development, 1989) 


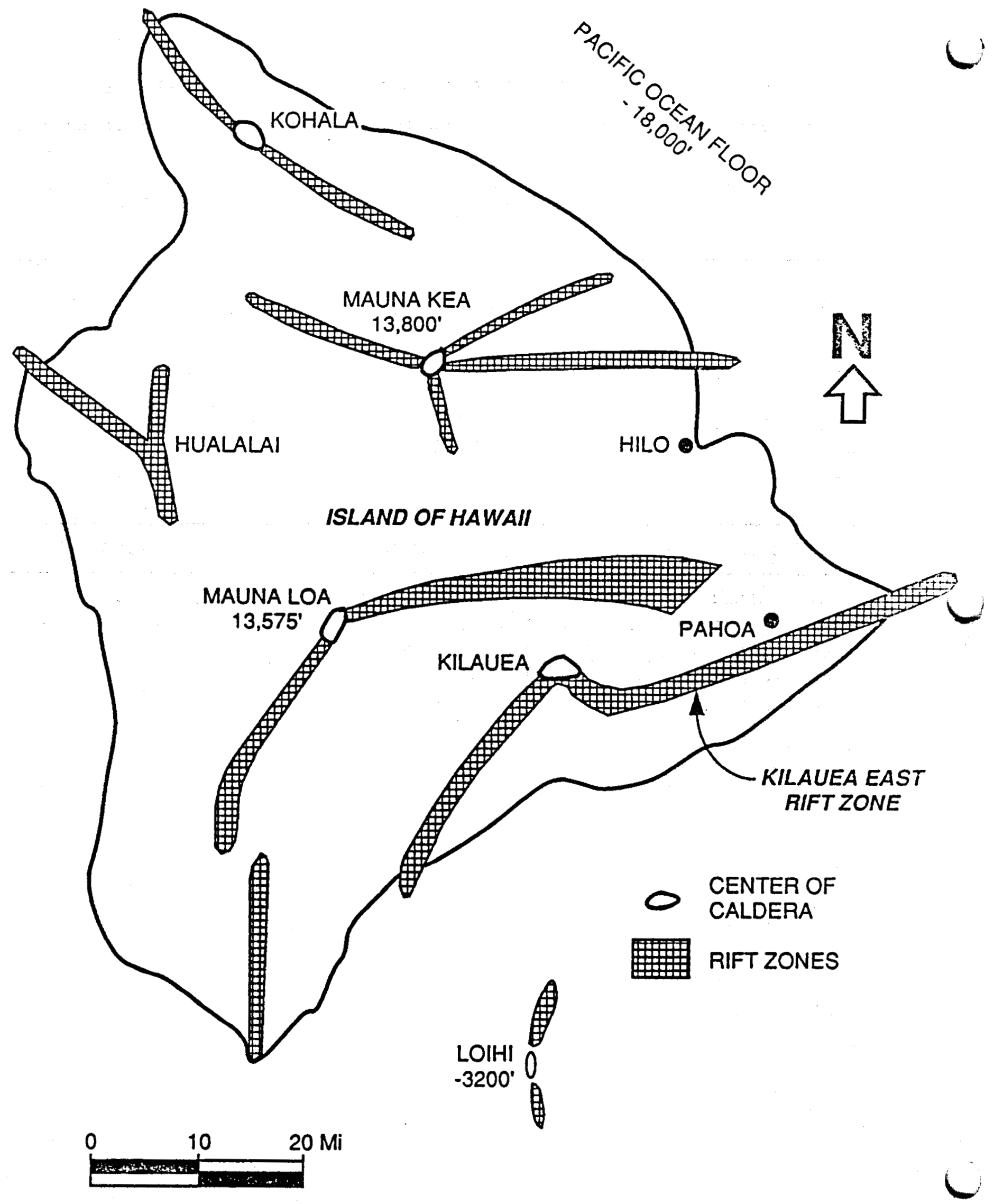

Figure A - 1 
C

C

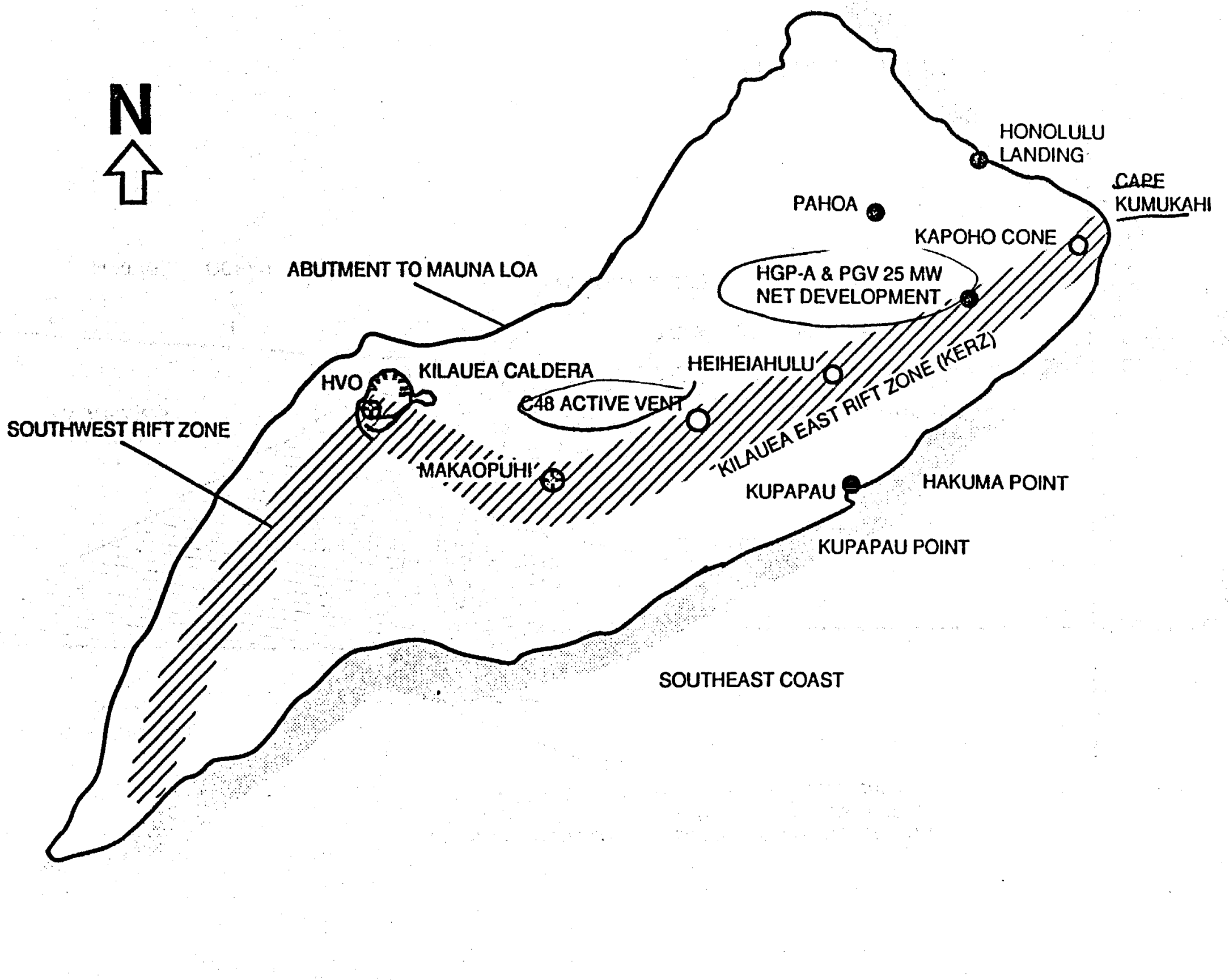




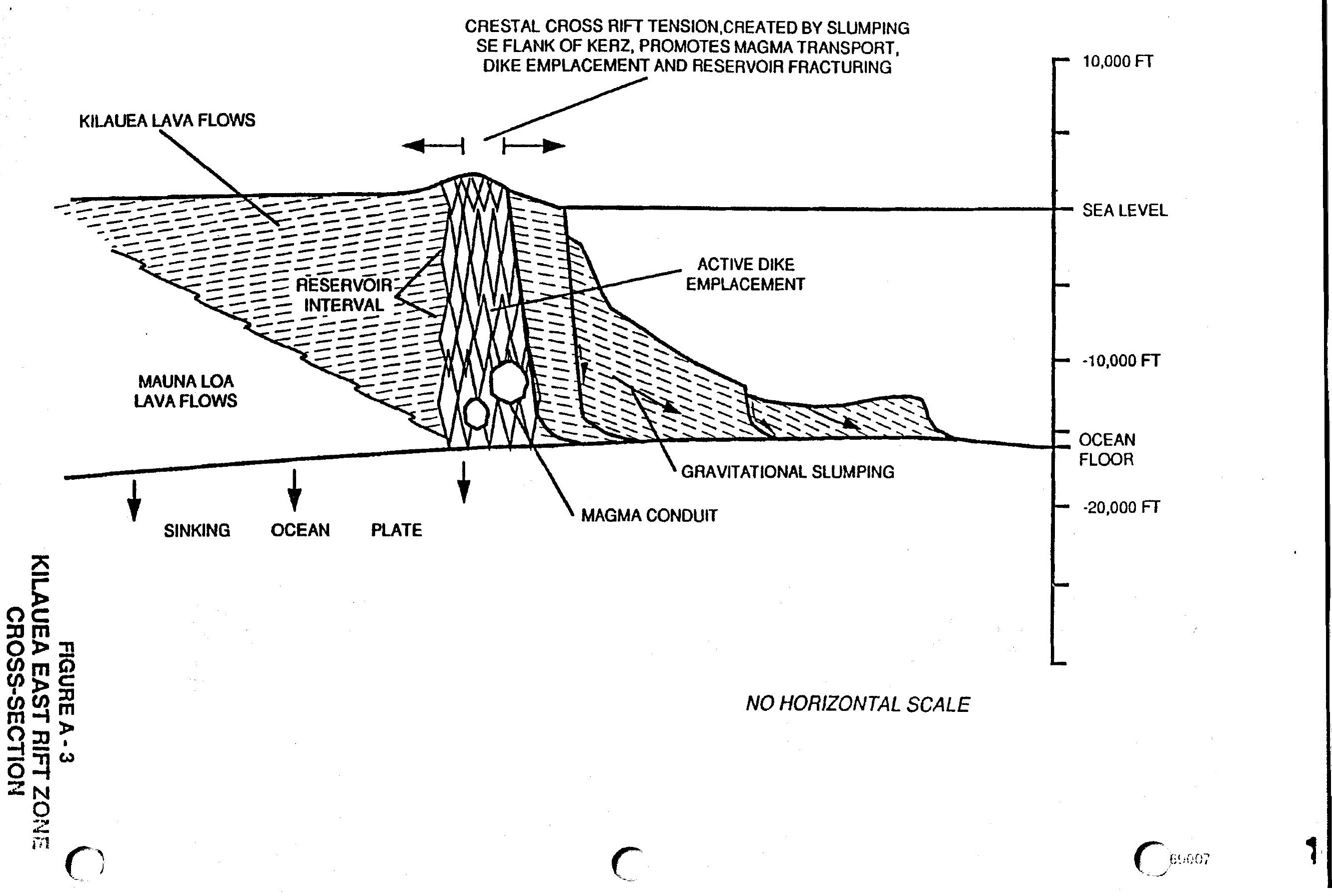


TEMPERATURE, IN DEGREES CELSIUS

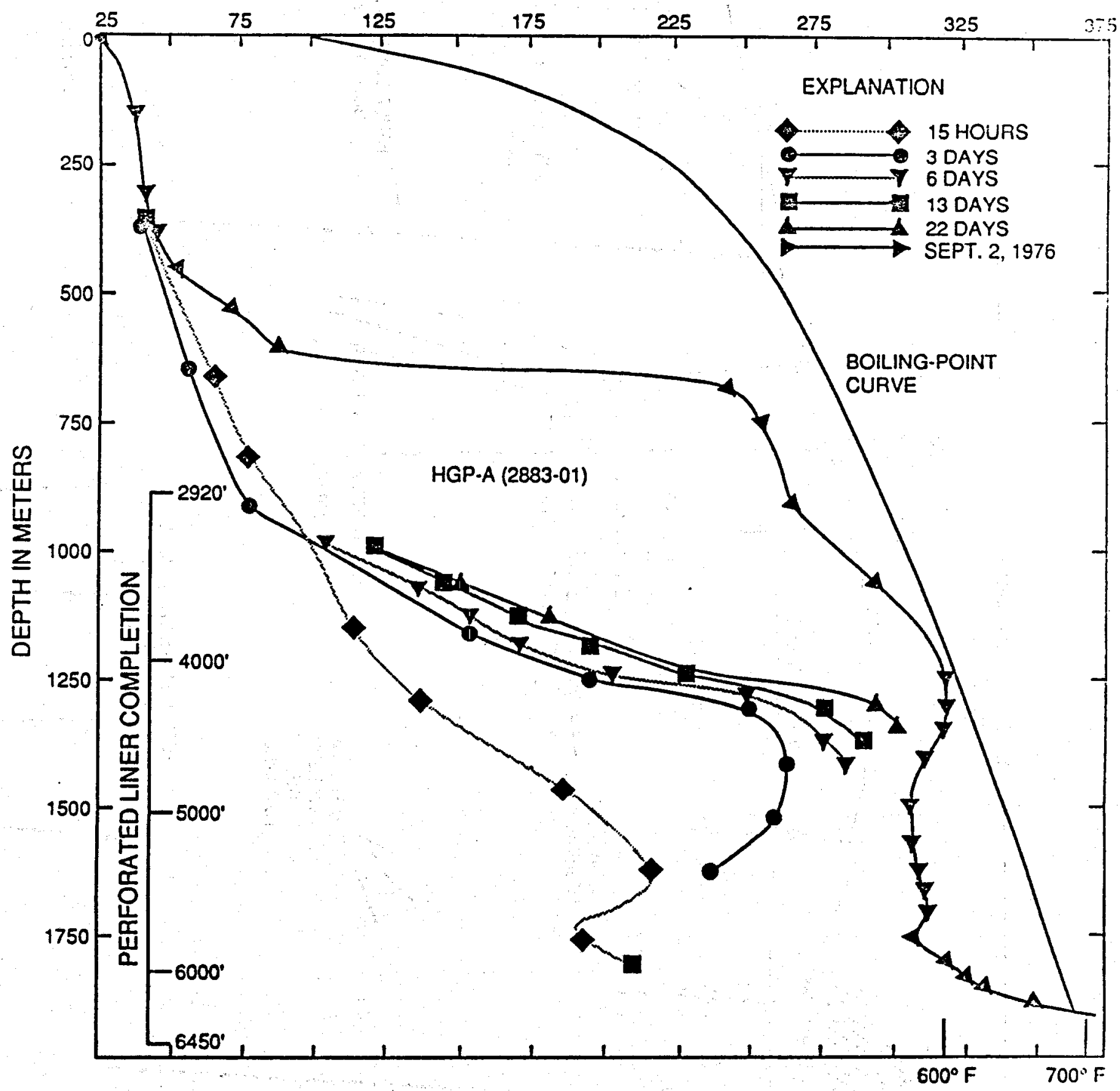




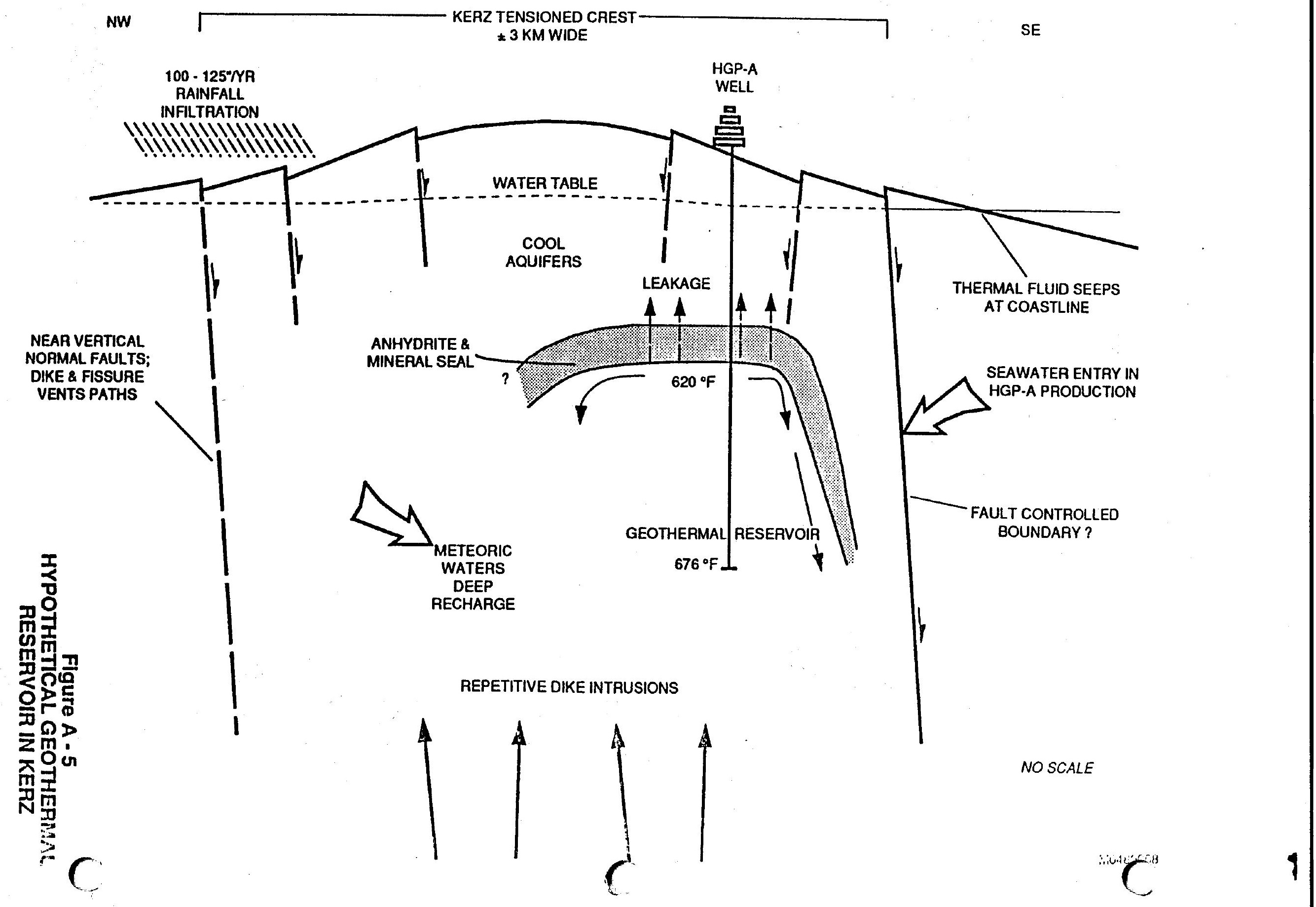




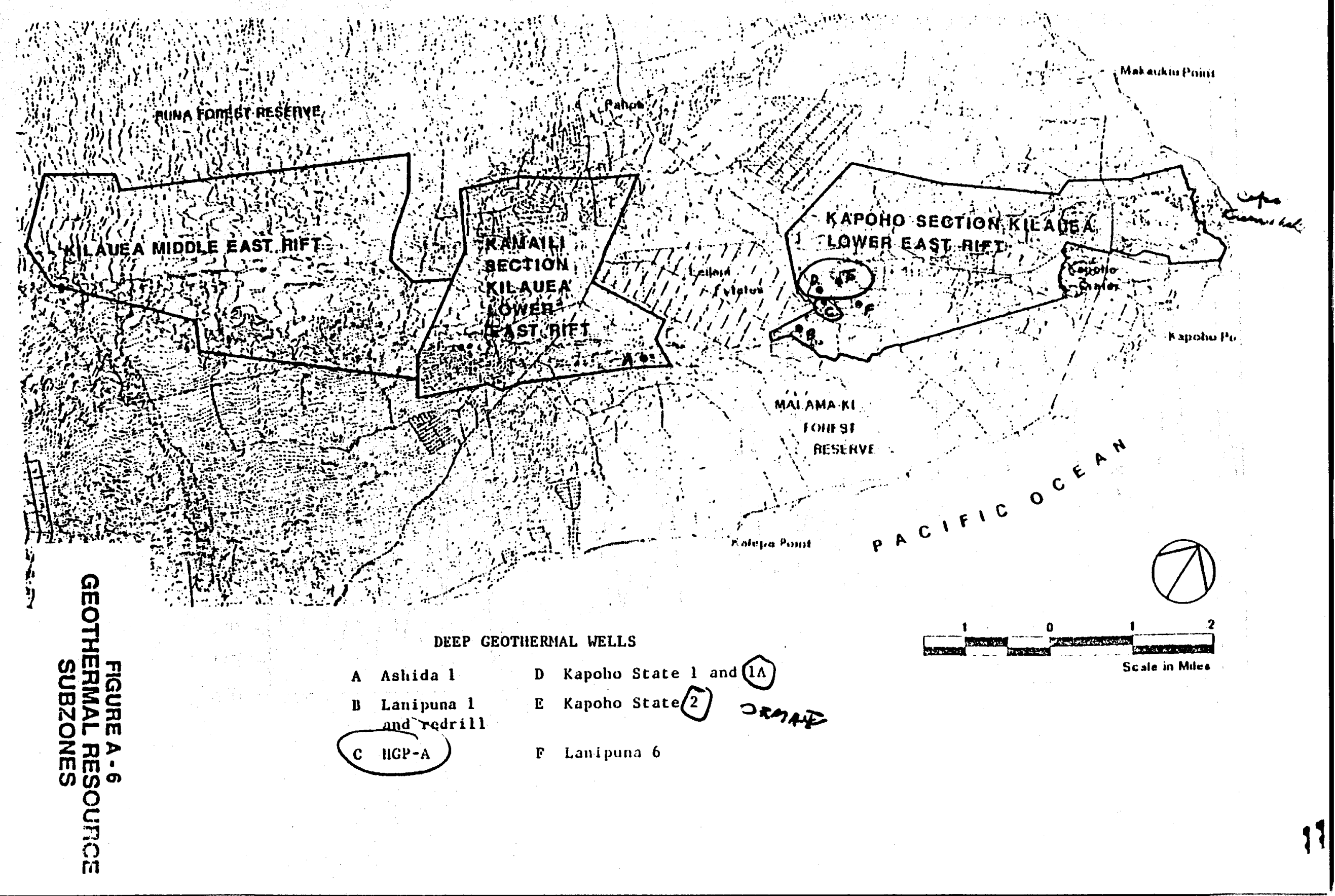




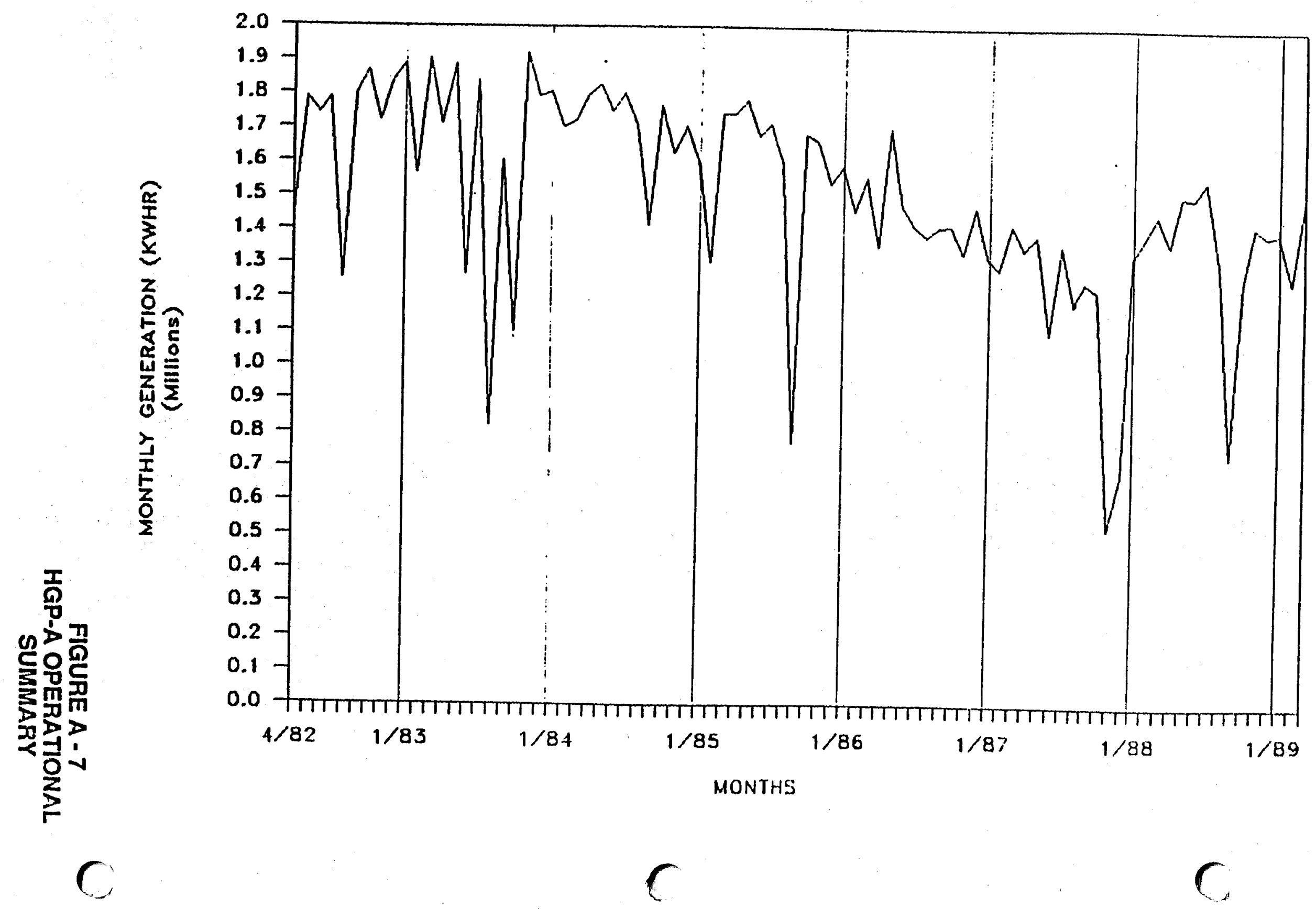




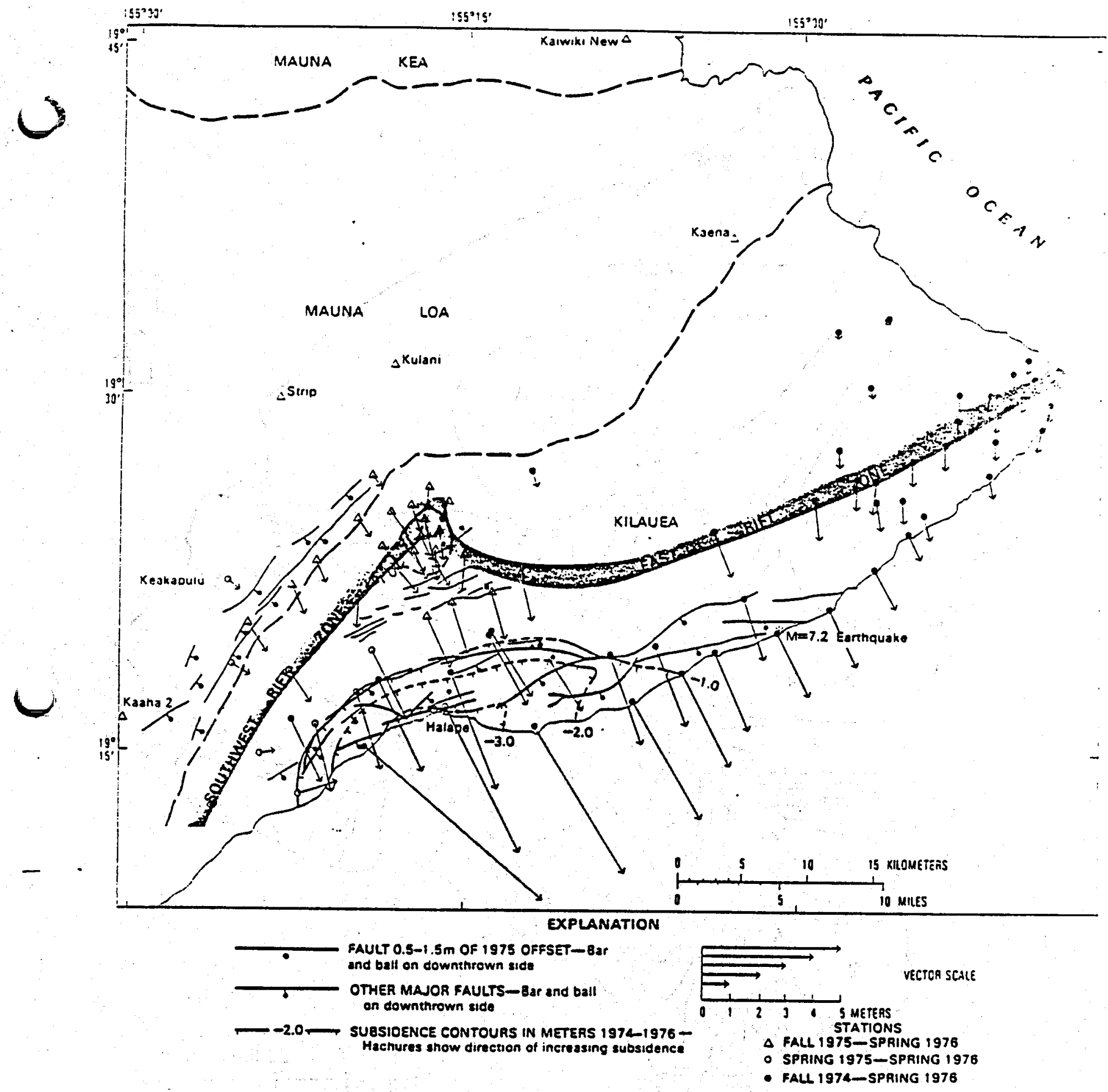

(From USGS Professional Paper 1276: Lipman, et al, 1985) 


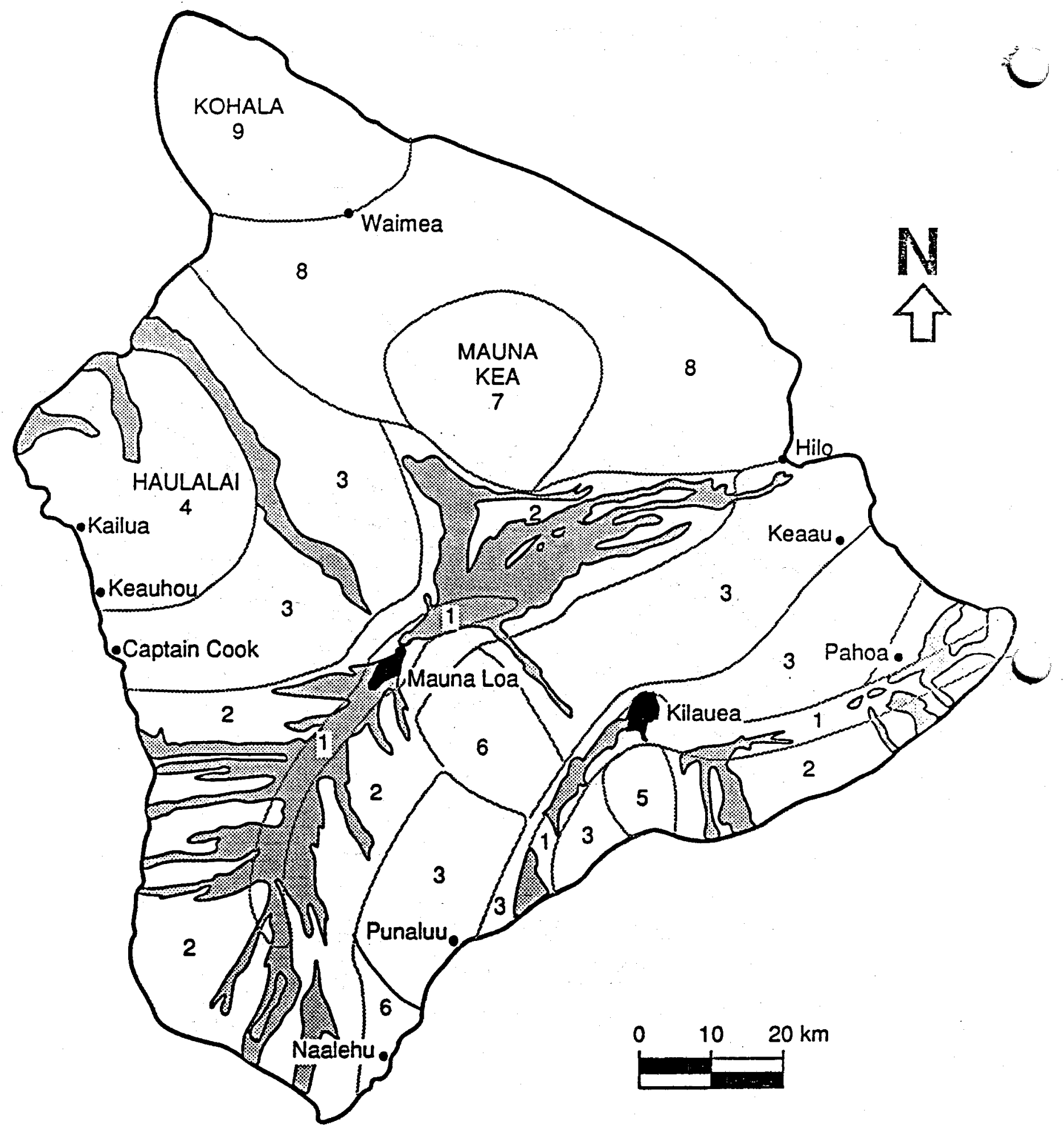

ZONES 1 - 9 IN ORDER OF DECREASING HAZARD 